\title{
Cross Section Measurements for Quasi-Elastic Neutrino-Nucleus Scattering with the MINOS Near Detector
}

\author{
Mark Edward Dorman \\ University College London
}

Submitted to University College London in fulfilment

of the requirements for the award of the degree of Doctor of Philosophy.

April 2008 


\section{Declaration}

I, Mark Dorman, confirm that the work presented in this thesis is my own. Where information has been derived from other sources, I confirm that this has been indicated in the thesis.

Mark Dorman 


\begin{abstract}
The Main Injector Neutrino Oscillation Search (MINOS) is a long baseline neutrino oscillation experiment based at the Fermi National Accelerator Laboratory (FNAL) in Chicago, Illinois. MINOS measures neutrino interactions in two large iron-scintillator tracking/sampling calorimeters; the Near Detector on-site at FNAL and the Far Detector located in the Soudan mine in northern Minnesota. The Near Detector has recorded a large number of neutrino interactions and this high statistics dataset can be used to make precision measurements of neutrino interaction cross sections.
\end{abstract}

The cross section for charged-current quasi-elastic scattering has been measured by a number of previous experiments and these measurements disagree by up to $30 \%$. A method to select a quasi-elastic enriched sample of neutrino interactions in the MINOS Near Detector is presented and a procedure to fit the kinematic distributions of this sample and extract the quasi-elastic cross section is introduced. The accuracy and robustness of the fitting procedure is studied using mock data and finally results from fits to the MINOS Near Detector data are presented. 


\section{Acknowledgements}

Firstly I would like to thank my family for their constant love and support throughout my time as a student (which is the best part of the last 25 years) and most especially to thank Michelle for her tireless encouragement, for keeping me sane at times when I had no right to be and for making me smile every day.

Many thanks also to Jason, Tom, Dan, Sarah and Shiva; my partners in crime on the adventure that has been my $\mathrm{PhD}$ and without whose collective brain power I would have no doubt encountered many more difficulties. There are a multitude of people who have helped me and made this work possible and I am hugely grateful to Jenny, Chris, Ryan, Trish, Geoff, Jeff, Costas and Alfons for all their advice and to all my UCL colleagues that I have pestered over the years. I am particularly indebted to Mike who has unerringly directed my research from my first plot though to the end of this thesis.

I have been fortunate enough to have worked in many countries and met a wide variety of people during my time as a $\mathrm{PhD}$ student and so to all those with whom I have shared a cabin, plane, road trip, technical discussion, coffee break, game of football or a pint - thank you.

Mark Dorman, Sunday 13th April, 2008 


\section{Contents}

1 Introduction 16

1.1 Theory of Neutrino Oscillations _ . . . . . . . . . . . . . . . . . 18

1.2 Measuring Neutrino Oscillations in MINOS . . . . . . . . . . . . . . . 22

1.3 Motivation for Cross Section Measurements . . . . . . . . . . . . . . . . . . 24

2 The Weak Interaction and Neutrino Physics 26

2.1 The Phenomenology of Weak Interactions . . . . . . . . . . . . . . . . 26

2.1.1 Fermi's Point-like Four-Fermion Theory of Nuclear $\beta$-Decay . . . . . . 26

2.1.2 Parity Violation and the V-A Structure of the Weak Interaction . . . . . 28

2.1.3 Chirality and Helicity . . . . . . . . . . . . . . . . . 31

2.1.4 The Glashow-Weinberg-Salam Electroweak Gauge Theory . . . . . . . 33

2.2 Neutrino-Nucleus Scattering _ . . . . . . . . . . . . . . . . 37

2.2.1 Neutrino-Nucleon Scattering . . . . . . . . . . . . . . . . 37

2.2.2 The Kinematics of Neutrino-Nucleon Scattering . . . . . . . . . . 38

2.2.3 The Quasi-Elastic Cross Section . . . . . . . . . . . . . . . . . . 40

2.2.4 Nuclear Effects . . . . . . . . . . . . . . . . . . . . . . . . 45

2.2.5 Deviations from the Dipole Form . . . . . . . . . . . . . . . 48

3 Measuring $M_{A}^{Q E L}+\mathbf{5 2}$

3.1 General Method for Extracting $M_{A}^{Q E L} \ldots \ldots \ldots \ldots \ldots \ldots$

3.2 Results from the Argonne 12-Foot Bubble Chamber . . . . . . . . . . . . . 53

3.3 Results from the K2K Scintillating Fiber Detector . . . . . . . . . . . . . . . 56

3.4 Results from the MiniBooNE Experiment . . . . . . . . . . . . . . . . 58

3.5 Summary of Current Knowledge and World-Average Values _ . . . . . . . . . 59

4 The MINOS Experiment $\quad 63$

4.1 The NuMI Beamline . . . . . . . . . . . . . . . . . . . . . 63

4.2 The MINOS Detectors _ . . . . . . . . . . . . . . . . . . 68

4.2.1 Common Features of the Detectors . . . . . . . . . . . . . . . 68

4.2 .2 The Far Detector . . . . . . . . . . . . . . . . . . . 70 
4.2 .3 The Near Detector . . . . . . . . . . . . . . . . . . . 72

4.2.4 The Calibration Detector . . . . . . . . . . . . . . . . . . . 74

4.3 MINOS Physics Goals _ . . . . . . . . . . . . . . . . . 75

5 Near Detector Data and Monte Carlo Simulation $\quad 81$

5.1 Event Reconstruction . . . . . . . . . . . . . . . . . . . . 81

5.2 Calibration of the MINOS Detectors . . . . . . . . . . . 83

5.3 Near Detector Data Quality . . . . . . . . . . . . . . . . . . 86

5.4 MINOS Monte Carlo Simulation . . . . . . . . . . . . . . . . . . . 90

6 Near Detector Event Selection $\quad 93$

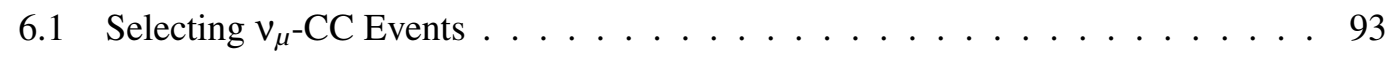

6.1.1 Beam and Hadron Production Parameter Reweighting . . . . . . . 100

6.1.2 Comparison of Data to Monte Carlo . . . . . . . . . . . . 105

6.2 Selecting $v_{\mu}$-CC QEL Events . . . . . . . . . . . . . . . 112

6.2.1 Comparison of Data to Monte Carlo . . . . . . . . . . . . . 114

6.3 Near Detector Kinematic Resolutions ． . . . . . . . . . . . . . . 117

6.3.1 Resolution Fitting Method . . . . . . . . . . . . . . . 117

6.3.2 Results for ND Kinematic Resolutions . . . . . . . . . . . . . . . . . 118

$7 M_{A}^{Q E L}$ Fit Procedure $\quad 124$

7.1 Overview of the Fit . . . . . . . . . . . . . . . 124

7.2 Fit Parameters . . . . . . . . . . . . . . . . . . 126

7.3 Fit Configurations . . . . . . . . . . . . . . . . . . 134

7.4 Fit Convergence Problems . . . . . . . . . . . . . . . 136

7.4.1 Convergence with MC Smearing . . . . . . . . . . . . . 138

7.5 Headline Fit Configurations . . . . . . . . . . . . . . . . . . . 141

7.5.1 Headline Fit Configuration I . . . . . . . . . . . . . . . . . 141

7.5.2 Headline Fit Configuration II . . . . . . . . . . . . . . . . . . . . 142

7.5.3 Example Results for Fit I . . . . . . . . . . . . . . . . . . 143

7.5.4 Example Results for Fit II . . . . . . . . . . . . . . . . 146

7.6 Studying the Fit . . . . . . . . . . . . . . . . . . . . 149 
$8 M_{A}^{Q E L}$ Measurement with ND Data 162

8.1 Headline Fits to the Data . . . . . . . . . . . . . . . . . . 162

8.2 Results for Data Fit Configuration I . . . . . . . . . . . . . . . . . 164

8.3 Results for Data Fit Configuration II . . . . . . . . . . . . . . . . . . . 177

8.4 Reconciling the Fit Results . . . . . . . . . . . . . . . . . 187

8.5 Summary and Final Result _ . . . . . . . . . . . . . . . . . . . . 190

8.6 Improvements for the Future . . . . . . . . . . . . . . . . . . . 192

9 Conclusion 196

References $\quad 199$

$\begin{array}{ll}\text { A Apparent Fit Bias Studies } & 203\end{array}$

B Correlation Coefficients $\quad 209$ 


\section{List of Figures}

1 Muon neutrino survival probability as a function of neutrino energy. . . . . . 21

2 Neutrino coherent forward scattering via the neutral current. . . . . . . . . 22

3 Electron neutrino coherent forward scattering via the charged current. . . . . . 22

4 Best fit Far Detector neutrino energy spectrum from the first year of MINOS

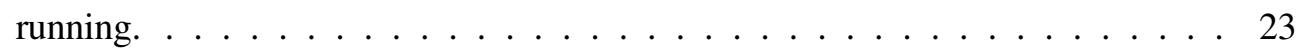

5 Best fit neutrino oscillation parameters and confidence intervals from the first year of MINOS running. . . . . . . . . . . . . . . . . . . . . . 24

6 Fermi's pointlike four-fermion interaction for neutron $\beta$-decay. . . . . . . . . 26

$7 \quad W^{+}$exchange process for $v_{e}+\mu^{-} \rightarrow e^{-}+v_{\mu} \ldots \ldots \ldots 36$

8 Kinematics of charged current neutrino-nucleus scattering. . . . . . . . . . . 39

9 Effect of $M_{A}^{Q E L}$ upon the shape of $d \sigma_{Q E L} / d Q^{2} \ldots \ldots \ldots \ldots$. . . . . . . 46

10 Effect of $M_{A}^{Q E L}$ upon the shape and normalisation of $d \sigma_{Q E L} / d Q^{2} \ldots \ldots$. . . . 46

11 Implications of moving from a free nucleon description of the nucleus to a Fermi Gas model. . . . . . . . . . . . . . . . . . . . . . . . . 48

12 Effect of moving from the Dipole Form Factors to the BBA-2003 Form Factors. 49

$13 v_{\mu}$-CC QEL and $\bar{v}_{\mu}$-CC QEL scattering cross sections. . . . . . . . . . 50

$14 Q^{2}$ distribution for QEL events in the Argonne 12-foot bubble chamber. . . . . 55

$15 Q^{2}$ distributions for QEL events in the K2K SciFi detector. . . . . . . . . . 58

$16 Q^{2}$ distribution for QEL events in the MiniBooNE detector. . . . . . . . . . . . 60

17 Overview of the MINOS experimental layout. . . . . . . . . . . . 63

18 Overhead view of the FNAL accelerator complex. . . . . . . . . . . . . 64

19 Photograph of the NuMI graphite target. . . . . . . . . . . . . . 65

20 Photographs of the NuMI parabolic focussing horns. . . . . . . . . . . . . 65

21 The components of the NuMI beamline. . . . . . . . . . . . . . . . 66

22 Weekly and integrated POT delivered by NuMI during it's first year of operation. 67

23 Photograph of a MINOS detector scintillator module. . . . . . . . . . . . . 69

24 Optical readout of a MINOS detector scintillator plane. . . . . . . . . . . . . 70

25 Photograph of the Far detector. . . . . . . . . . . . . . 71

26 Photograph of the Near detector. . . . . . . . . . . . . . . . 73 
27 Diagram of the sections of the Near detector. . . . . . . . . . . . . . . 74

28 Photograph of the Calibration detector. . . . . . . . . . . . . . 75

29 MINOS sensitivity to the atmospheric neutrino oscillation parameters. . . . . . 76

30 MINOS sensitivity to $\theta_{13}$ as a function of the CP-violating phase factor $\delta$. . . . 77

31 Example of $v_{\mu}$ oscillations into sterile neutrinos in MINOS. . . . . . . . . 78

32 Measurements of the inclusive $\mathrm{CC} v+N$ scattering cross section. . . . . . . 79

33 MINOS sensitivy to the proton structure function $F_{2}\left(x, Q^{2}\right) \ldots \ldots \ldots$

34 Activity in the ND during a single beam spill. . . . . . . . . . . . . . 82

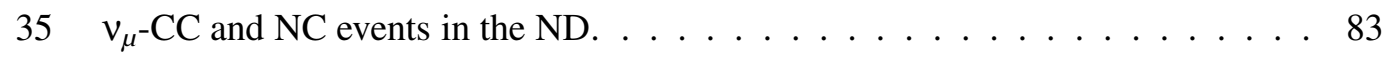

$36 \%$ change in ND and FD response to through-going cosmic-ray muons with time. 84

37 Effect of calibration procedure upon the mean signal per strip per muon crossing. 85

38 Calorimeter response to electrons and pions in data and Monte Carlo simulation. 87

39 Reconstructed CC-like events per unit exposure in the ND as a function of time. 88

40 Mean reconstructed neutrino, muon and shower energies and track vertex positions for CC-like events in the ND as a function of time. . . . . . . . . . . . . 89

41 Stability of CC-like neutrino energy spectrum in the ND as a function of month of data taking. . . . . . . . . . . . . . . . . . . . . . . 89

42 NEUGEN cross sections for inclusive $\mathrm{v}_{\mu}$-CC scattering, quasi-elastic scattering and single pion production as a function of neutrino energy. . . . . . . . . . 91

43 Data and MC comparisons of quantities used to construct a parameter to separate $\mathrm{CC}$ and $\mathrm{NC}$ events. . . . . . . . . . . . . . . . . . . . 97

44 Data and MC comparison of the $\mathrm{CC} / \mathrm{NC}$ event separation parameter. . . . . . . 98

45 Efficiency and purity of the CC-like sample with a cut on the $\mathrm{CC} / \mathrm{NC}$ separation parameter. . . . . . . . . . . . . . . . . . . 99

46 Data and MC comparisons of the reconstructed neutrino energy in 3 different beam configurations. . . . . . . . . . . . . . . 100

47 Pion yields from the NuMI target as a function of pion $p_{z}$ and $p_{T} . \ldots 101$

48 Parametric fits to the predicted target hadron production yields as given by




49 Errors on the ND neutrino energy spectrum coming from beam optics/focussing effects. . . . . . . . . . . . . . . . . . . 104

50 Scale factors for target-produced $\pi^{+}$as a function of $p_{z}$ and $p_{T} \ldots \ldots \ldots$

51 Reconstructed track vertex positions along the $x$-axis in data, nominal MC and tuned MC . . . . . . . . . . . . . . . . . . 106

52 Reconstructed track vertex positions along the $y$-axis in data, nominal MC and tuned MC . . . . . . . . . . . . . . . . . . . . . 107

53 Reconstructed track vertex positions along the $z$-axis in data, nominal MC and tuned MC . . . . . . . . . . . . . . . . . . . . 107

54 Reconstructed $\theta_{\mu}$ for CC-like events in data, nominal MC and tuned MC. . . . 108

55 Reconstructed $E_{\mu}$ for CC-like events in data, nominal MC and tuned MC. . . . 109

56 Reconstructed $E_{s h w}$ for CC-like events in data, nominal MC and tuned MC. . . 110

57 Reconstructed $E_{\mathrm{v}}$ for CC-like events in data, nominal MC and tuned MC. . . 110

58 Reconstructed $Q^{2}$ for CC-like events in data, nominal MC and tuned MC. . . . 111

59 Reconstructed $W^{2}$ for CC-like events in data, nominal MC and tuned MC. . . . 111

60 QEL-like samples in MC selected using two different methods. . . . . . . . . . 113

61 Efficiency and purity of the QEL-like event sample as a function of $E_{v}$ and $Q^{2} . \quad 114$

62 Reconstructed $E_{\mathrm{v}}^{Q E L}$ for QEL-like events in data, nominal MC and tuned MC. . 116

63 Reconstructed $Q_{Q E L}^{2}$ for QEL-like events in data, nominal MC and tuned MC. . 116

64 Reconstructed minus true $Q^{2}$ in slices of true $Q^{2}$. . . . . . . . . . . 118

65 ND resolutions and pulls on the resolutions for the muon energy as measured by range in the detector. . . . . . . . . . . . . . . . . . 120

66 ND resolutions and pulls on the resolutions for the muon scattering angle in the detector. . . . . . . . . . . . . . . . . . . . . . 121

67 ND resolutions and pulls on the resolutions for the squared four-momentum transfer in the detector. . . . . . . . . . . . . . . . . . 123

68 Effect of up to $\pm 20 \%$ changes to the value of $M_{A}^{Q E L}$ upon the $Q_{Q E L}^{2}$ and $E_{\mathrm{v}}^{Q E L}$ distributions of QEL-like events in MC . . . . . . . . . . . . . . 125

69 Effect of $\pm 2 \%$ changes to the muon energy scale on the $Q_{Q E L}^{2}$ and $E_{\mathrm{v}}^{Q E L}$ distributions for QEL-like events in MC. . . . . . . . . . . . . . . 127 
70 Comparisons of the track length of $2 \mathrm{GeV}$ muons for data and $\mathrm{MC}$ events in

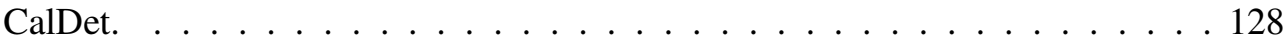

71 Mean $Q^{2}$ and $E_{v}$ values in slices of reconstructed shower energy for CC-like events in data and nominal MC. . . . . . . . . . . . . . . . . . . . 129

72 Effect of $\pm 35 \mathrm{MeV}$ changes to the hadronic energy offset on the $Q_{Q E L}^{2}$ and $E_{\mathrm{v}}^{Q E L}$ distributions for QEL-like events in MC . . . . . . . . . . . . . . 130

73 Effect of $\pm 10 \%$ changes to the background scale on the $Q_{Q E L}^{2}$ and $E_{\mathrm{v}}^{Q E L}$ distributions for QEL-like events in MC . . . . . . . . . . . . . . . . . . . 131

74 Effect of $\pm 8 \%$ changes to the neutrino flux for events with $E_{v}^{\text {true }} \in[3.0,4.0)$ $\mathrm{GeV}$ on the $Q_{Q E L}^{2}$ and $E_{\mathrm{v}}^{Q E L}$ distributions for QEL-like events in MC. . . . . . 132

75 Mean $Q^{2}$ in slices of neutrino energy for QEL-like events in data and nominal

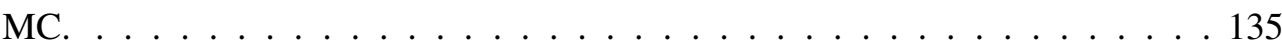

76 Example of a discontinuous $\chi^{2}$ surface. . . . . . . . . . . . . . 137

77 Schematic of MC smearing procedure . . . . . . . . . . . . . . . 138

78 Projections of $\Delta \chi^{2}$ with MC smearing applied. . . . . . . . . . . . . . . 140

79 Ratios of smeared to unsmeared MC . . . . . . . . . . . . . . . . . . . . 140

80 Example results for mock data fit I. . . . . . . . . . . . . . . . . . . 144

81 Fit contours for example fit I to mock data. . . . . . . . . . . . . . . . . . 145

82 Example results for mock data fit II. . . . . . . . . . . . . . . . . . . . 147

83 Fit contours for example fit II to mock data. . . . . . . . . . . . . . . . . 148

84 Mock data fits results with a variety of different $E_{\text {had }}$ cut values. . . . . . . . 152

85 Mock data fit results from investigation into apparent fit bias. . . . . . . . . . . 154

86 Mock data fit results with a variety of ranges and binning in $Q_{Q E L}^{2} \ldots \ldots$

87 Best fit minus truth values for 30 shape and normalisation fits to the $Q_{Q E L}^{2}$ and $E_{\mathrm{v}}^{Q E L}$ distributions in mock data. . . . . . . . . . . . . . . . . 157

88 Projections of the 30 best fit minus truth parameter values. . . . . . . . . . . 158

89 Best fit minus truth values for 100 shape-only fits to the $Q_{Q E L}^{2}$ distribution in mock data. . . . . . . . . . . . . . . . . . . . . . . . 160

90 Projections of the 100 best fit minus truth parameter values. . . . . . . . . . . 161

$91 Q_{Q E L}^{2}$ distributions for a fit to the data with configuration I. . . . . . . . . 165 
92 Two-dimensional fit contours for the $M_{A}^{Q E L}$-scale versus each of the three systematic parameters. . . . . . . . . . . . . . . 166

93 Two-dimensional fit contours for permutations of the three systematic parameters. 167

94 Comparisons of squared four-momentum transfer for data, nominal MC and best fit MC . . . . . . . . . . . . . . . . . . . . . 168

95 Comparisons of neutrino energy for data, nominal MC and best fit MC. . . . . 169

96 Two-dimensional ratios of the data to the nominal MC and to the best fit MC. . 169

97 POT-normalised data, nominal and best fit $\mathrm{MC} Q_{Q E L}^{2}$ distributions from a fit with $E_{v}^{Q E L} \in[2.0,3.0) \mathrm{GeV}$. . . . . . . . . . . . . . . . . . 172

$98 \chi^{2}$-surfaces for grid searches using events with all neutrino energies and those in a slice from $2.0 \rightarrow 3.0 \mathrm{GeV} \ldots \ldots \ldots$

99 Zoom in on the $\chi^{2}$-surface from a grid search using events with neutrino energies in a slice from $2.0 \rightarrow 3.0 \mathrm{GeV} . \ldots \ldots$. . . . . . . . . . . . . . . . .

$100 Q_{Q E L}^{2}$ and $E_{\mathrm{v}}^{Q E L}$ distributions for a fit to the data with configuration II. . . . . . 179

101 Comparisons of data, nominal $\mathrm{MC}$ and best fit $\mathrm{MC}$ as a function of $E_{\mathrm{v}}^{Q E L}$ and

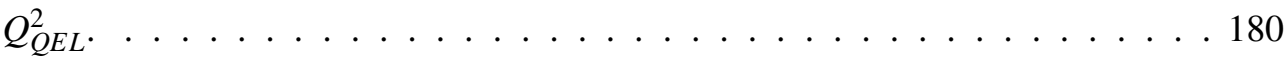

102 Two-dimensional ratios of the data to the nominal MC and to the best fit MC. . 180

103 Two-dimensional fit contours for the $M_{A}^{Q E L}$-scale versus each of the three nonflux factor systematic parameters. . . . . . . . . . . . . . . 181

104 Two-dimensional fit contours for permutations of the three non-flux factor systematic parameters. . . . . . . . . . . . . . . . . 182

105 Two-dimensional fit contours for a selection of flux factors. . . . . . . . . . . 186

106 Two-dimensional fit contours as a function of the $2 \rightarrow 3 \mathrm{GeV}$ flux factor and each of the non-flux factor systematic parameters. . . . . . . . . . . . 187

$1071 \sigma, 2 \sigma$ and $3 \sigma$ confidence interval contours for headline fit I. . . . . . . . . 191

108 Reconstructed $Q_{Q E L}^{2}$ for the $\mathrm{MC}$ and mock data used in the apparent bias studies with mock data sub-sample 1. . . . . . . . . . . . . . . . . . . 204

109 Reconstructed $Q_{Q E L}^{2}$ for the MC and mock data used in the apparent bias studies with mock data sub-sample 2 . . . . . . . . . . . . . . . . . . 204 
110 Reconstructed $Q_{Q E L}^{2}$ for the MC and mock data used in the apparent bias studies with mock data sub-sample $3 \ldots$. . . . . . . . . . . . . . . 205

111 Mock data fit results from the investigation into the apparent fit bias using mock data sample 1. . . . . . . . . . . . . . . . . . . . 207

112 Mock data fit results from the investigation into the apparent fit bias using mock

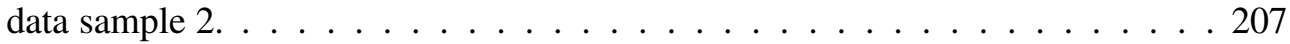

113 Mock data fit results from the investigation into the apparent fit bias using mock

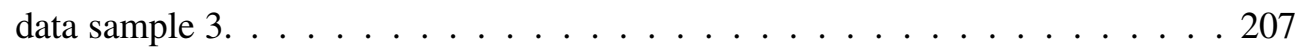




\section{List of Tables}

1 Possible forms of the weak current allowed in the Dirac theory. . . . . . . . . 30

2 Calculation of kinematic quantities for charged current neutrino-nucleus scat-



$3 M_{A}^{Q E L}$ results from the Argonne 12 -foot bubble chamber. . . . . . . . . . 55

4 Summary of previous measurements of $M_{A}^{Q E L} \ldots \ldots \ldots \ldots \ldots$. . . . . 61

5 Definitions of NuMI beam configurations and corresponding exposures. . . . . 67

6 Numbers of selected CC-like events per 1e20 POT in nominal MC broken down by true interaction type. . . . . . . . . . . . . . . . . . . . 99

7 Numbers of selected QEL-like events per 1e20 POT in nominal MC broken down by true interaction type. . . . . . . . . . . . . . . 115

8 Summary of results for example fit I to mock data. . . . . . . . . . . . . . . . 143

9 Summary of results for example fit II to mock data. . . . . . . . . . . . . . . 146

10 RMS values for the distributions of best fit minus truth parameter values from 30 mock data fits with configuration II. . . . . . . . . . . . . . . . . 159

11 RMS values for the distributions of best fit minus truth parameter values from 100 mock data fits with configuration I. . . . . . . . . . . . . . . . 160

12 Best fit parameter values for a fit to the data with configuration I. . . . . . . . . 164

13 Results for data fit configuration I with and without the beam and hadron production tuning weights applied to the MC . . . . . . . . . . . . . 170

14 Results for data fit configuration I using events from the full $E_{v}^{Q E L}$ distribution and in a number of independant slices of $E_{\mathrm{v}}^{Q E L} \ldots \ldots \ldots \ldots \ldots$

15 Results for data fit configuration I using events from the full $E_{\mathrm{v}}^{Q E L}$ distribution and for the repeated fit considering events with neutrino energies between 2.0 and $3.0 \mathrm{GeV} . \ldots \ldots \ldots \ldots \ldots \ldots$

16 Results for data fit configuration I using different QEL-like sample hadronic energy cuts. . . . . . . . . . . . . . . . . . . 175

17 Best fit parameter values for a fit to the data with configuration II. . . . . . . . 178

18 Results for data fit configuration II with and without the beam and hadron production tuning weights applied to the MC . . . . . . . . . . . . . 184 
19 Results for data fit configuration II with the QEL-like sample hadronic energy cut at $200 \mathrm{MeV}$ and $300 \mathrm{MeV} . \ldots \ldots$

20 Results for data fit configuration I with a series of tigher constraints upon the muon energy scale. . . . . . . . . . . . . . . . . . . . . . . . . 189

21 Results for data fit configuration II with a series of tigher constraints upon the muon energy scale. . . . . . . . . . . . . . . . . . . 190

22 Fit results for bias studies using four statistically independant sub-samples of

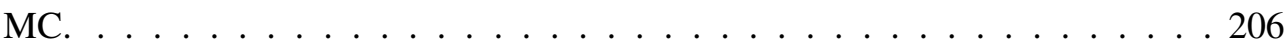




\section{Introduction}

The ubiquitous Standard Model of Particle Physics has been extremely successful in describing the interactions and properties of the fundamental particles and forces present in nature. It's predictions have been tested many times by a succession of more and more powerful and precise physics experiments and no serious deviations have been found. There are, however, areas in which the Standard Model cannot provide an adequate framework. Most notable is the inclusion of a description of the force of gravity although perhaps a more active area of current research is the field of neutrino physics.

Of the twelve elementary fermionic particles in the Standard Model there are three flavours of neutrino; the electron, muon and tau neutrinos $\left(v_{e}, v_{\mu}\right.$ and $\left.v_{\tau}\right)$. In the Standard Model the

neutrinos are massless, spin- $\frac{1}{2}$ leptons that carry no electromagnetic or colour charge and are only able to interact via the weak nuclear force. Neutrinos were first postulated by Wolfgang Pauli [1] as a solution to the problem of the continuous energy spectrum of the electrons emitted in nuclear $\beta$-decays. In the classical two-body decay $\left(n \rightarrow p+e^{-}\right)$energy and momentum could not be conserved unless the energy of the emitted electron took a discrete value but the addition of a third undetected particle, that carried away some fraction of the energy, restored this most famous of conservation laws. It was not until 26 years later, in 1956, that Frederick Reines and Clyde Cowan directly observed neutrinos interacting in a large tank of Cadmium-laced water near the Savannah River nuclear plant in Augusta, Georgia [2].

The phenomenology of weak interactions has come a long was since Pauli's 'Dear Radioactive Ladies and Gentlemen' letter of 1930 and Fermi's point-like four fermion model of nuclear $\beta$-decay but there are still experimental results that cannot be explained within the Standard Model. One such set of observations became known as the atmospheric neutrino anomaly and show a discrepancy in the numbers of electron and muon neutrinos arriving at the Earth's surface. Cosmic rays incident on the upper atmosphere interact with molecular nucleons and produce a cascade of secondary particles including large numbers of pions. These pions predominantly decay to muons with the following decay chain: 


$$
\begin{gathered}
\pi^{-} \rightarrow \mu^{-}+\bar{v}_{\mu} \\
\mu^{-} \rightarrow e^{-}+v_{e}+\bar{v}_{\mu}
\end{gathered}
$$

Equations 1 and 2 (and their charge conjugates) suggest that the ratio of $v_{\mu}\left(\overline{v_{\mu}}\right): v_{e}\left(\bar{v}_{e}\right)$ arriving should be 2 : 1, however, both water Čerenkov experiments such as IMB [3] and Kamiokande II [4] and iron sampling calorimeter experiments such as Soudan 2 [5] have measured a deficit in the numbers of $v_{\mu}$. A solution, suggested by Bruno Pontecorvo in 1967 [6], was the possibiltity of neutrino flavour mixing analagous to the phenomenon of flavour changing weak decays for the quarks. Pontecorvo postulated that if neutrinos had a finite mass and that the neutrino weak eigenstates, which participate in the weak interaction, were not the same as the neutrino mass eigenstates, which propagate through space, then a mixing matrix could be formed in a similar way to the Cabbibo-Kobayashi-Maskawa (CKM) matrix of the quark sector. Consequently a neutrino born of a particular flavour in a weak interaction will become a superposition of all the flavours as the mass eigenstates propagate and at some time later can be detected in another weak interaction as a neutrino of a different flavour. This phenomenon, known as neutrino oscillations, can explain the apparent loss of atmospheric $v_{\mu}$ and it's subsequent discovery by the Super-Kamiokande collaboration in 1998 [7] required at least one massive neutrino and a theory beyond the Standard Model.

Neutrino oscillations have been investigated by various experiments over the last ten years and limits on the parameters governing the oscillations have been set. Experiments study neutrinos produced in the cosmic ray interactions mentioned above, neutrinos produced in the nuclear reactions taking place in our Sun or neutrinos produced in nuclear power plants across the globe. One limitation on the accurate measurement of oscillations, stemming from the use of such sources, is that these experiments do not have direct control over the creation of the neutrinos. Another experimental possibility is to use a beam of neutrinos produced at a particle accelerator and regulated with a specialised neutrino beamline. This is the approach being employed by the Main Injector Neutrino Oscillation Search (MINOS) experiment, currently running at the Fermi National Accelerator Laboratory (FNAL) near Chicago, Illinois. 
The primary physics goal of MINOS is to make an accurate measurement of the parameters governing the oscillations of $v_{\mu} \nrightarrow v_{\mu}$ and the collaboration has already published it's first results [8]. MINOS uses the Neutrinos at the Main Injector (NuMI) facility to produce a neutrino beam, comprised almost entirely of $v_{\mu}$, and samples the beam composition and neutrino energy spectrum with two large iron-scintillator tracking/sampling calorimeters; the Near Detector on-site at FNAL and the Far Detector located in the Soudan Underground Laboratory, $734 \mathrm{~km}$ away in northern Minnesota. The most sensitive measurement of the neutrino oscillation parameters that MINOS can make is based on comparing the neutrino energy spectrum at the two detectors; once at the Near Detector before the neutrinos have had a chance to oscillate and then again at the Far Detector after oscillations have occured. The following sections will present the theory of neutrino oscillations, describe in further detail the oscillation parameter measurement technique used by MINOS and then finally motivate the analysis to be presented in this thesis.

\subsection{Theory of Neutrino Oscillations}

In the Standard Model neutrinos are not given mass but it is now known, through the discovery of neutrino oscillations, that this cannot be true. Neutrino masses can be generated in the Standard Model but this requires additions to the framework and as such neutrino oscillations are the first evidence for physics 'beyond the Standard Model'.

Neutrino oscillations stem from the idea that the weak eigenstates of the neutrinos, in which neutrinos are detected, are different from the mass eigenstates, which propagate through space. These two 'bases' are related through a unitary mixing matrix, analagous to the CKM matrix of the quark sector, called the Pontecorvo-Maki-Nakagawa-Sakata (PMNS) [6][9] matrix. A neutrino produced in a weak interaction will consist of a linear superposition of the mass eigenstates $v_{i}$ :

$$
\left|v_{\alpha}\right\rangle=\sum_{i} V_{\alpha i}^{*}\left|v_{i}\right\rangle
$$

where $V_{\alpha i}$ are elements of the PMNS matrix. To understand the origin of neutrino oscillations (in vacuum) consider a neutrino that is initially in the state $v_{\alpha}$ as defined in equation 3 and apply 
the Schrödinger equation to the $i^{\text {th }}$ component in its rest frame to see that the time evolution of this initial state component is:

$$
\left|v_{i}\left(\tau_{i}\right)\right\rangle=e^{-i m_{i} \tau_{i}}\left|v_{i}(0)\right\rangle
$$

where $m_{i}$ is the mass of $v_{i}$ and $\tau_{i}$ the time in the frame of that component. This Lorentz invariant phase factor may be re-written in terms of the time $t$ and position $L$ in the laboratory frame and the energy $E_{i}$ and momentum $p_{i}$ of the $v_{i}$ component in this frame:

$$
\exp \left(-i m_{i} \tau_{i}\right)=\exp \left(-i\left(E_{i} t-p_{i} L\right)\right)=\exp \left(-i\left(E_{i}-p_{i}\right) L\right)
$$

where equation 5 follows as the neutrino is, in practice, highly relativistic with $t \approx L$. Then assuming a definite common momentum $p$ for all the components of $v_{\alpha}$ and $m_{i} \ll p$ :

$$
E_{i}^{2}=p^{2}+m_{i}^{2} \Rightarrow E_{i} \approx p+m_{i}^{2} / 2 p
$$

Equations 3 to 6 can be combined and then using $E \approx p$ as the average energy of the components of $v_{\alpha}$ :

$$
\left|v_{\alpha}(L)\right\rangle \approx \sum_{i} V_{\alpha i}^{*} e^{-i\left(m_{i}^{2} / 2 E\right) L}\left|v_{i}\right\rangle
$$

Then using the unitarity of the PMNS matrix to invert equation 3 and inserting the result into equation 7 yields:

$$
\left|v_{\alpha}(L)\right\rangle \approx \sum_{\beta}\left[\sum_{i} V_{\alpha i}^{*} e^{-i\left(m_{i}^{2} / 2 E\right) L} V_{\beta i}\right]\left|v_{\beta}\right\rangle
$$

It can be seen from equation 8 that a neutrino born of flavour $\alpha$ and travelling a distance $L$ will become a superposition of all the neutrino flavours. The probability, $P\left(v_{\alpha} \rightarrow v_{\beta}\right)$, of this $v_{\alpha}$ being of flavour $\beta$ after travelling a distance $L$ is given by $\left|\left\langle v_{\beta} \mid v_{\alpha}(L)\right\rangle\right|^{2}$. Using the unitarity of the MNS matrix and equation 8 this may be written: 


$$
\begin{aligned}
P\left(v_{\alpha} \rightarrow v_{\beta}\right)=\delta_{\alpha \beta} & -4 \sum_{i>j} \mathcal{R}\left(V_{\alpha i}^{*} V_{\beta i} V_{\alpha j} V_{\beta j}^{*}\right) \sin ^{2}\left[1.27 \Delta m_{i j}^{2}(L / E)\right] \\
& +2 \sum_{i>j} I\left(V_{\alpha i}^{*} V_{\beta i} V_{\alpha j} V_{\beta j}^{*}\right) \sin \left[2.54 \Delta m_{i j}^{2}(L / E)\right]
\end{aligned}
$$

where $\Delta m_{i j}^{2} \equiv m_{i}^{2}-m_{j}^{2}$ and the sine terms come from the relation:

$$
\Delta m_{i j}^{2}(L / 4 E) \simeq 1.27 \Delta m_{i j}^{2}\left(\mathrm{eV}^{2}\right) \frac{L(\mathrm{~km})}{E(\mathrm{GeV})}
$$

In the case that only two mass eigenstates (and hence two linear combinations of flavour eigenstates) and a single mass splitting, $\Delta m^{2}$, are important equation 9 can be simplified to:

$$
P\left(v_{\alpha} \rightarrow v_{\beta}\right) \simeq 4\left|\sum_{i \pm} V_{\alpha i}^{*} V_{\beta i}\right|^{2} \sin ^{2}\left[1.27 \Delta m^{2}(L / E)\right] \quad(\alpha \neq \beta)
$$

where the notation $i \pm$ means a sum over those mass eigenstates that lie above or below $\Delta m^{2}$. Such situations can arise when, for example, the charged lepton that is produced along with the subject neutrino for a particular experiment is only coupled to significantly by two mass eigenstates. This is the case for the MINOS experiment where the muon type neutrinos produced by the NuMI beam are only coupled to significantly by the $v_{2}$ and $v_{3}$ neutrino mass eigenstates. For such 'quasi-two-neutrino oscillations' [10] the mixing of the flavour eigenstates is given by:

$$
\left(\begin{array}{c}
v_{\alpha} \\
v_{\beta}
\end{array}\right)=\left(\begin{array}{cc}
\cos \theta & \sin \theta \\
-\sin \theta & \cos \theta
\end{array}\right)\left(\begin{array}{c}
v_{1} \\
v_{2}
\end{array}\right)
$$

where $\theta$ is the mixing angle and using the relation:

$$
4\left|\sum_{i \pm} V_{\alpha i}^{*} V_{\beta i}\right|^{2}=\sin ^{2} 2 \theta
$$

the probability for a neutrino to oscillate from the initial state $v_{\alpha}$ to the state $v_{\beta}$ after travelling a distance $L$ is: 


$$
P\left(v_{\alpha} \rightarrow v_{\beta}\right)=\sin ^{2} 2 \theta \sin ^{2}\left[1.27 \Delta m^{2}(L / E)\right]
$$

Equation 14 shows that for experiments, such as MINOS, that have a fixed ${ }^{1}$ ratio of $L / E$ the amount of oscillation is dependant on just two parameters, the mixing angle $\theta$ and the mass splitting $\Delta m^{2}$. Figure 1 shows the survival probability of a $v_{\mu}$ as a function of energy using the form of equation 14 , the MINOS baseline of $735 \mathrm{~km}, \sin ^{2} 2 \theta=1$ (maximal mixing) and $\Delta m^{2}=2.74 \times 10^{-3}$ [11]. MINOS is sensitive to the disappearance of neutrinos at $\sim 2 \mathrm{GeV}$ corresponding to the first dip (from the right) in the probability function shown in figure 1 .

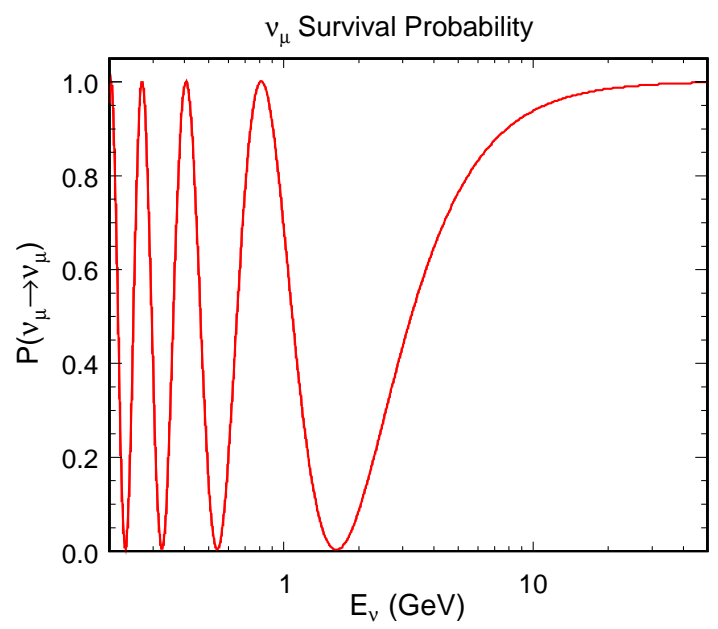

Figure 1: Muon neutrino survival probability as a function of neutrino energy.

The above derivations considered neutrinos travelling through a vacuum. The implications of the passage of a neutrino through matter for its probabilities to oscillate to other flavours are known as the Mikheyev-Smirnov-Wolfenstein (MSW) effect [12][13]. The MSW effect considers the coherent forward scattering of neutrinos from particles they encounter as they traverse a medium. Each of the $v_{e}, v_{\mu}$ and $v_{\tau}$ can undergo a coherent forward scatter via the neutral current $\left(Z^{0}\right.$ exchange) process shown in figure 2 but the $v_{e}$ can also undergo an

\footnotetext{
${ }^{1}$ The neutrinos are in general not mono-energetic but have an energy spectrum that is peaked at a certain value that depends upon the running conditions of the experiment.
} 
additional charged current $\left(W^{ \pm}\right.$exchange) coherent foward scatter via the interactions shown in figure 3.

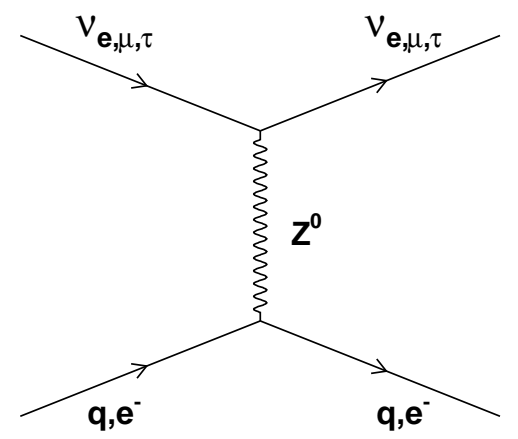

Figure 2: Neutral current coherent forward scattering diagram for neutrino interactions with matter.
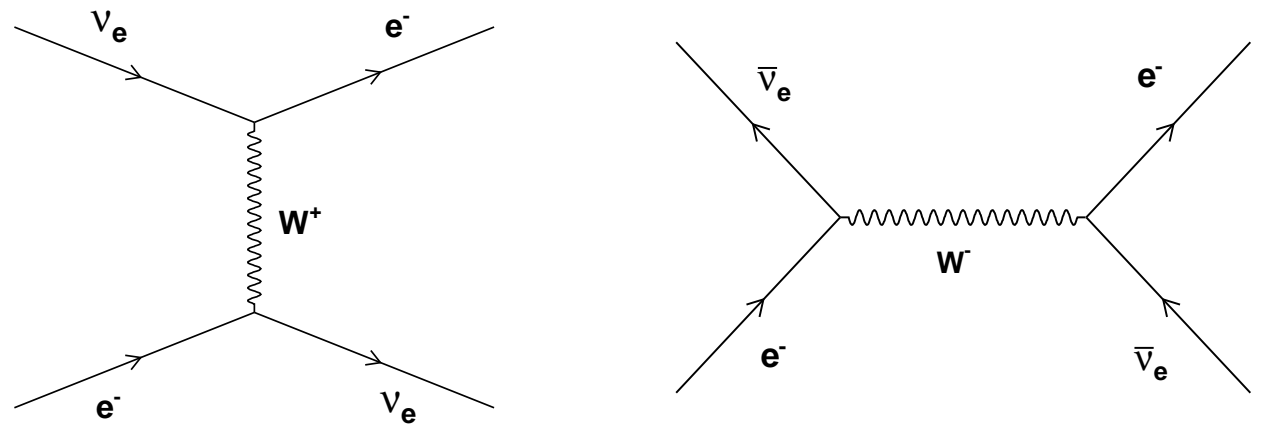

Figure 3: Charged current coherent forward scattering diagrams for the interations of electron neutrinos (left-hand diagram) and anti-neutrinos (right-hand diagram) with matter.

The MSW effect is of great importance for the study of solar neutrinos due to the high electron density of the sun but has little effect for the MINOS experiment which uses a beam comprised, almost entirely, of muon neutrinos.

\subsection{Measuring Neutrino Oscillations in MINOS}

The signature for neutrino oscillations in MINOS is an energy dependant disappearance of $\nu_{\mu}$ at the Far Detector, relative to the expectation assuming no oscillations, in accordance with the structure of the $v_{\mu}$ survival probability function shown in figure 1. MINOS uses the data from the Near Detector to validate the Monte Carlo simulation of the experiment and then extrapolates the Near Detector neutrino energy spectrum, as predicted by the simulation, to 
the corresponding neutrino energy spectrum at the Far Detector. This prediction assumes that none of the $v_{\mu}$ have oscillated into another flavour of neutrino and can be compared to the Far Detector data. Deviations between the Far Detector data and the predicted un-oscillated spectrum can then be fit to extract the neutrino oscillation parameters; $\Delta m^{2}$ and the mixing angle $\theta$.

Figure 4 shows the reconstructed neutrino energy spectrum for interactions in the Far Detector recorded during the first year of MINOS data taking as well as the predicted un-oscillated spectrum, as extrapolated from the Near Detector via a number of different methods, and the best fit Monte Carlo simulated energy spectrum assuming the oscillation hypothesis. Figure 4 also shows the ratio of data to the expectation from the Monte Carlo simulation as a function of the reconstructed neutrino energy and this ratio exhibits a 'dip' structure indicative of the presence of neutrino oscillations.
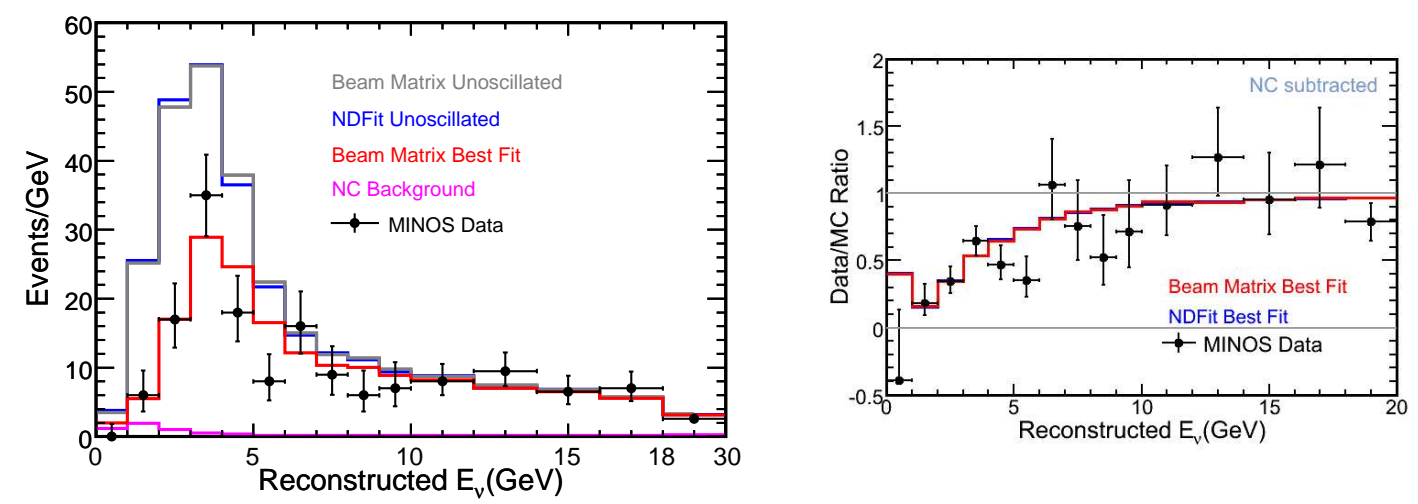

Figure 4: The left-hand figure shows reconstructed neutrino energy spectra for MINOS Far Detector data, un-oscillated Monte Carlo simulation and best fit Monte Carlo simulation assuming the neutrino oscillation hypothesis. The right-hand figure shows the ratio of the data to the Monte Carlo expectation as a function of reconstructed neutrino energy and exhibits an energy dependant loss of $\nu_{\mu}$ events.

Figure 5 shows the best fit neutrino oscillation parameters extracted from the MINOS Far Detector data along with the $68 \%$ and $90 \%$ confidence intervals for this measurement. It also shows the $90 \%$ confidence intervals corresponding to measurements made by the $\mathrm{K} 2 \mathrm{~K}$ and Super-Kamiokande collaborations. Figures 4 and 5 are taken from [11] and show that, using data from only the first year of running, MINOS has already made a competitive measurement 
of the neutrino oscillation parameters.

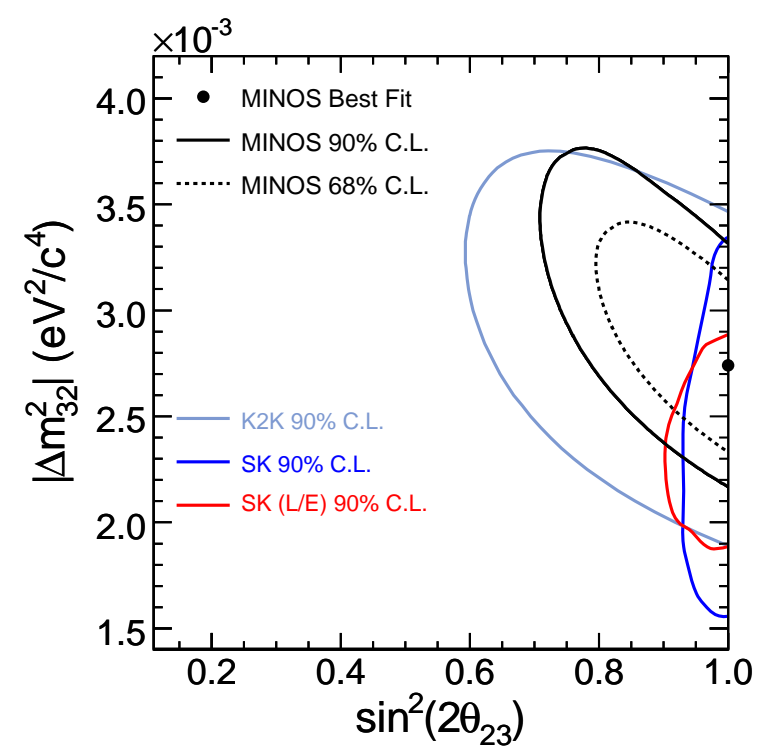

Figure 5: Best fit neutrino oscillation parameters and confidence intervals corresponding to an analysis of MINOS Far Detector data taken during the first year of running. The subscripts on the oscillation parameters $\Delta m_{32}^{2}$ and $\sin ^{2}\left(2 \theta_{23}\right)$ denote the neutrino mass eigenstates involved in the oscillation.

\subsection{Motivation for Cross Section Measurements}

The MINOS neutrino oscillation analysis relies on a knowledge of the event rate in the detectors and this event rate is a product of the incident neutrino flux from the NuMI beam and the neutrino interaction cross sections. Neutrino interaction cross sections are not well known at lower neutrino energies $\left(E_{\mathrm{v}}<10 \mathrm{GeV}\right)$ with uncertainties on the cross sections for certain processes, such as quasi-elastic scattering, at the level of $20 \%$ to $30 \%$.

An improved understanding of both the inclusive and exclusive cross sections for neutrino interactions is both important for the analysis of MINOS data and interesting in it's own right. For quasi-elastic scattering, which is the dominant interaction type for neutrino energies below $\sim 1.5 \mathrm{GeV}$, there have been many previous measurements in disagreement with each other.

Increased accuracy for neutrino cross section measurements is also important for the next gen- 
eration of neutrino experiments, such as Nova and T2K, that hope to measure a second mixing angle, $\theta_{13}$, which is responsible for the sub-dominant oscillations of $v_{\mu}$ to $v_{e}$. These experiments are going to be searching for a small effect and will have to tightly control their systematic error, to which the uncertainty on interaction cross sections will certainly contribute.

Finally, as will be demonstrated in the coming chapters, a study of quasi-elastic scattering requires and can provide a large amount of other interesting information; (i) the NuMI neutrino flux must be considered in a cross section analysis and an improved understanding of the NuMI beam is advantageous for all MINOS physics analyses, (ii) quasi-elastic interactions probe the axial nature of the nucleon in a way to which only neutrinos experiments have access and (iii) in the MINOS detectors the $v_{\mu}$ are not interacting with free nucleons but with steel nuclei and quasi-elastic interactions are sensitive to the, currently not well modelled, details of the nuclear system. 


\section{The Weak Interaction and Neutrino Physics}

\subsection{The Phenomenology of Weak Interactions}

\subsubsection{Fermi's Point-like Four-Fermion Theory of Nuclear $\beta$-Decay}

Four years after Pauli first postulated the existence of the neutrino Enrico Fermi proposed the first quantum field theory for the weak interaction. Analagous to the emission of photons in nuclear $\gamma$-decay Fermi considered the neutrino-electron pair to be created and emitted in the nuclear transition of a neutron to a proton (as shown in figure 6).



Figure 6: Fermi's pointlike four-fermion interaction picture of neutron $\beta$-decay.

Motivated again by quantum electrodynamics, Fermi considered the interaction to happen at a single spacetime point and to involve a weak 4-vector 'current' between the neutron and proton. To make the interaction Lorentz invariant Fermi also included a current between the electon and anti-neutrino and constructed a 'current-current' interaction amplitude given by:

$$
\frac{G_{F}}{\sqrt{2}} \bar{u}_{p} \gamma^{\mu} u_{n} \bar{u}_{e^{-}} \gamma_{\mu} u_{\bar{v}_{e}}=\frac{G_{F}}{\sqrt{2}} j_{N}^{\mu} j_{l}^{\mu}
$$

where $G_{F}$ is Fermi's constant $\left(G_{F}=1.166 \times 10^{-5} \mathrm{GeV}^{-2}\right), u$ and $\bar{u}$ are Dirac spinors (mathematical repesentations of the quantum state of a particle), $\gamma^{\mu}$ are the Dirac matrices, $\mu$ is a four-component index that labels time and spatial dimensions, $j_{N}^{\mu}$ denotes the nucleon current and $j_{l}^{\mu}$ denotes the leptonic current. In the Dirac respresentation the $\gamma^{\mu}$ are defined by the following matrices: 


$$
\begin{aligned}
\gamma^{0}=\left(\begin{array}{cccc}
1 & 0 & 0 & 0 \\
0 & 1 & 0 & 0 \\
0 & 0 & -1 & 0 \\
0 & 0 & 0 & -1
\end{array}\right) & \gamma^{1}=\left(\begin{array}{cccc}
0 & 0 & 0 & 1 \\
0 & 0 & 1 & 0 \\
0 & -1 & 0 & 0 \\
-1 & 0 & 0 & 0
\end{array}\right) \\
\gamma^{2}=\left(\begin{array}{cccc}
0 & 0 & 0 & -i \\
0 & 0 & i & 0 \\
0 & i & 0 & 0 \\
-i & 0 & 0 & 0
\end{array}\right) & \gamma^{3}=\left(\begin{array}{cccc}
0 & 0 & 1 & 0 \\
0 & 0 & 0 & -1 \\
-1 & 0 & 0 & 0 \\
0 & 1 & 0 & 0
\end{array}\right)
\end{aligned}
$$

At some points in this chapter field theory notation (where $\psi(\mathbf{x}, t)$ denotes a quantum field) will also be used and using this notation equation 15 can be written as a local interaction density according to:

$$
\frac{G_{F}}{\sqrt{2}} \overline{\hat{\psi}}_{p}(\mathbf{x}, t) \gamma^{\mu} \hat{\psi}_{n}(\mathbf{x}, t) \overline{\hat{\psi}}_{e^{-}}(\mathbf{x}, t) \gamma_{\mu} \hat{\psi}_{\bar{v}}(\mathbf{x}, t)
$$

Fermi's current-current formalism was successful in describing many of the characteristics of nuclear $\beta$-decay but it was unable to account for nucleon spin 'flip' (transitions where the nuclear spin changes by one unit). A further breakthrough came in 1936 when Gamow and Teller introduced a more general four-fermion interaction in which they allowed bilinear terms (terms with two indices), such as shown in equation 18, whose presence in the currents accomodated the observed nucleon spin flips.

$$
\bar{u}_{p} \sigma_{\mu v} u_{n} \quad \text { where } \quad \sigma_{\mu v}=\frac{i}{2}\left(\gamma_{\mu} \gamma_{v}-\gamma_{v} \gamma_{\mu}\right)
$$

Over the next twenty years both the muon and pion were discovered and a wealth of weak interaction data was collected that could not be adequately explained by any of the proposed forms for the weak interaction. The solution was suggested in 1956 in a pioneering paper by Lee and Yang [14] who questioned the conservation of parity (inversion of spatial coordinates) in weak decays. It is now known that the weak interaction maximally violates parity but at that 
time parity was thought to be a fundamental property of all the interactions in nature and had been implicitly built into the proposed structure of the weak interaction.

\subsubsection{Parity Violation and the V-A Structure of the Weak Interaction}

Parity is the inversion of spatial coordinates and allows for a definition of polar vectors, pseudovectors (or axial vectors), scalars and pseudoscalars. A polar vector, $\mathbf{V}$, is one that transforms in the same way as the coordinate $\mathbf{x}$ under the parity operator, $\mathbf{P}$ :

$$
\mathbf{P}: \mathbf{x} \rightarrow-\mathbf{x} \quad, \quad \mathbf{P}: \mathbf{V} \rightarrow-\mathbf{V}
$$

and common examples of polar vectors are velocity, momentum and the electromagnetic current. Axial vectors transform in the same way as the vector product of two polar vectors and do no change sign under the parity operator:

$$
\mathbf{P}: \mathbf{U} \times \mathbf{V} \rightarrow(-\mathbf{U}) \times(-\mathbf{V})=\mathbf{U} \times \mathbf{V} \quad, \quad \mathbf{P}: \mathbf{A} \rightarrow \mathbf{A}
$$

and both angular momentum $(\mathbf{l}=\mathbf{x} \times \mathbf{p})$ and spin are examples of axial vectors. Scalars do not change sign under the parity operator as can be seen when forming from the dot-product of two polar vectors:

$$
\mathbf{P}: \mathbf{U} \cdot \mathbf{V} \rightarrow(-\mathbf{U}) \cdot(-\mathbf{V})=\mathbf{U} \cdot \mathbf{V}
$$

whereas pseudoscalars do reverse sign under the parity operator and could be formed from the triple scalar product of three polar vectors:

$$
\mathbf{P}: \mathbf{U} \cdot(\mathbf{V} \times \mathbf{W}) \rightarrow-\mathbf{U} \cdot(\mathbf{V} \times \mathbf{W})
$$

It can be shown that, under the parity operator, the free particle solution to the Dirac equation;

$$
\psi(\mathbf{x}, t)=N\left(\begin{array}{c}
\phi \\
\frac{\sigma \cdot \mathbf{p}}{E+m} \phi
\end{array}\right) e^{-i E t+i \mathbf{p} \cdot \mathbf{x}}
$$


where $\phi$ is a two-component Dirac spinor and $N$ a normalisation factor, has the following transformation:

$$
\mathbf{P}: \psi(\mathbf{x}, t) \rightarrow \psi_{P}(\mathbf{x}, t)=\beta \psi(-\mathbf{x}, t)
$$

where $\beta=\gamma^{0}$. As such a unitary quantum field operator, $\hat{\mathbf{P}}$, can be introduced such that:

$$
\psi_{P}(\mathbf{x}, t)=\hat{\mathbf{P}} \psi(\mathbf{x}, t) \hat{\mathbf{P}}^{-1}=\beta \psi(-\mathbf{x}, t)
$$

and possible forms of the weak interaction can be considered under the effect of this parity operator. For example consider the spatial parts of Fermi's weak 4-vector current:

$$
\begin{aligned}
\overline{\hat{\psi}}_{1 \mathbf{P}}(\mathbf{x}, t) \gamma^{\mu} \hat{\psi}_{2 \mathbf{P}}(\mathbf{x}, t) & =\hat{\psi}_{1 \mathbf{P}}^{\dagger}(\mathbf{x}, t) \beta \gamma^{\mu} \hat{\psi}_{2 \mathbf{P}}(\mathbf{x}, t) \\
& =\hat{\psi}_{1}^{\dagger}(-\mathbf{x}, t) \beta \beta \gamma^{\mu} \beta \hat{\psi}_{2}(-\mathbf{x}, t) \\
& =-\overline{\hat{\psi}}_{1}(-\mathbf{x}, t) \gamma^{\mu} \hat{\psi}_{2}(-\mathbf{x}, t) \\
\text { as } \beta \gamma^{\mu} & =-\gamma^{\mu} \beta, \beta^{2}=1
\end{aligned}
$$

Equation 26 shows that the spatial components of the 4-vector current transform like a polar vector under parity. A similar exercise shows that the $\mu=0$ time component transforms as a scalar under parity and as such Fermi's 4-vector currents do not allow for parity violation in the weak interaction. To accomodate parity violation the interaction must have terms that transform like axial vectors so as to produce both pseudoscalars and scalars. For Dirac particles these terms are introduced via the $\gamma_{5}$ matrix:

$$
\gamma_{5}=i \gamma^{0} \gamma^{1} \gamma^{2} \gamma^{3} \quad \text { and } \quad\left\{\gamma_{5}, \gamma_{\mu}\right\}=0 \quad \text { for } \mu \in\{0,1,2,3\}
$$

Equation 28 shows that a current involving $\gamma_{5}$ transforms as a pseudoscalar under parity and illustrates that the inclusion of $\gamma_{5}$ allows for parity violation in the weak interaction: 


\begin{tabular}{|c|c|c|}
\hline Interaction Type & Current Form & Parity \\
\hline \hline Scalar & $\overline{\hat{\psi}} \hat{\psi}$ & Even \\
\hline Pseudoscalar & $\overline{\hat{\psi}} \gamma_{5} \hat{\psi}$ & Odd \\
\hline Vector & $\hat{\hat{\psi}} \gamma^{\mu} \hat{\psi}$ & Odd \\
\hline Axial Vector & $\overline{\hat{\psi}} \gamma^{\mu} \gamma_{5} \hat{\psi}$ & Even \\
\hline Tensor & $\overline{\hat{\psi}} \frac{i}{2}\left(\gamma^{\mu} \gamma^{v}-\gamma^{v} \gamma^{\mu}\right) \hat{\psi}$ & Odd \\
\hline Pseudotensor & $\hat{\hat{\psi}} \frac{i}{2}\left(\gamma^{\mu} \gamma^{\nu}-\gamma^{v} \gamma^{\mu}\right) \gamma_{5} \hat{\psi}$ & Even \\
\hline
\end{tabular}

Table 1: Possible forms of the weak interaction allowed in the Dirac theory.

$$
\begin{aligned}
\overline{\hat{\psi}}_{1 \mathbf{P}}(\mathbf{x}, t) \gamma_{5} \hat{\psi}_{2 \mathbf{P}}(\mathbf{x}, t) & =\hat{\psi}_{1 \mathbf{P}}^{\dagger}(\mathbf{x}, t) \beta \gamma_{5} \hat{\psi}_{2 \mathbf{P}}(\mathbf{x}, t) \\
& =\hat{\psi}_{1}^{\dagger}(-\mathbf{x}, t) \beta \beta \gamma_{5} \beta \hat{\psi}_{2}(-\mathbf{x}, t) \\
& =-\overline{\hat{\psi}}_{1}(-\mathbf{x}, t) \gamma_{5} \hat{\psi}_{2}(-\mathbf{x}, t) \quad \text { as } \beta \gamma_{5}=-\gamma_{5} \beta
\end{aligned}
$$

It can be shown that currents of the form $\overline{\hat{\psi}}_{1}(\mathbf{x}, t) \gamma^{\mu} \gamma_{5} \hat{\psi}_{2}(\mathbf{x}, t)$ transform as axial 4-vectors under parity (with the $\mu=0$ components transforming like a pseudoscalar and the spatial parts transforming as an axial vector). Table 1 summarises the possible forms for the weak interaction allowed in the Dirac theory and notes how they transform under parity.

Parity violation was discovered in 1957 by $\mathrm{Wu}$ et al [15] in the nuclear $\beta$-decays of ${ }^{60} \mathrm{Co}$ and shortly after it was realised that Fermi's current-current interaction involved a combination of V-type (vector) and A-type (axial-vector) currents. A 'V-A' (vector minus axial) structure was proposed for the weak interaction which involved replacing Fermi's original 4-vector currents according to the following (where the Dirac spinor notation is again used):

$$
\bar{u}_{e^{-}} \gamma_{\mu} u_{v} \rightarrow \bar{u}_{e^{-}} \gamma_{\mu}\left(1-\gamma_{5}\right) u_{v}
$$

The V-A structure of the weak interaction is fundamental to the Standard Model and has the profound implication that only the left-handed components of fermions and the right-handed components of anti-fermions participate in the weak interaction. 


\subsubsection{Chirality and Helicity}

The helicity operator is defined as the projection of the spin of a particle in the direction of motion:

$$
\frac{1}{2} \frac{\boldsymbol{\sigma} \cdot \mathbf{p}}{|\mathbf{p}|}=\frac{1}{2} \boldsymbol{\sigma} \cdot \hat{\mathbf{p}}
$$

and chirality, which is an underlying characteristic of the weak interaction, can be expressed as the sign of the helicity operator. Particles with negative chirality are said to be left-handed whilst particles with positive chirality are said to be right-handed. In the Pauli-Dirac representation of the gamma matrices:

$$
\gamma_{5}=\left(\begin{array}{ll}
0 & I_{2} \\
I_{2} & 0
\end{array}\right)
$$

and using the (fermionic) Dirac spinor solutions from equation 23:

$$
\gamma_{5}\left(\begin{array}{l}
u_{a} \\
u_{b}
\end{array}\right)=\left(\begin{array}{ll}
0 & I_{2} \\
I_{2} & 0
\end{array}\right)\left(\begin{array}{c}
\phi \\
\frac{\sigma \cdot \mathbf{p}}{E+m} \phi
\end{array}\right)=\left(\begin{array}{c}
\frac{\sigma \cdot \mathbf{p}}{E+m} \phi \\
\phi
\end{array}\right)
$$

As the mass of the particle tends to zero then $E \rightarrow|\mathbf{p}|$ and:

$$
\gamma_{5}\left(\begin{array}{c}
u_{a} \\
u_{b}
\end{array}\right)=\left(\begin{array}{c}
(\boldsymbol{\sigma} \cdot \hat{\mathbf{p}}) \phi \\
\phi
\end{array}\right)=\left(\begin{array}{c}
(\boldsymbol{\sigma} \cdot \hat{\mathbf{p}}) \phi \\
(\boldsymbol{\sigma} \cdot \hat{\mathbf{p}})^{2} \phi
\end{array}\right)
$$

which implies that:

$$
\gamma_{5}\left(\begin{array}{l}
u_{a} \\
u_{b}
\end{array}\right)=\left(\begin{array}{cc}
\boldsymbol{\sigma} \cdot \hat{\mathbf{p}} & 0 \\
0 & \boldsymbol{\sigma} \cdot \hat{\mathbf{p}}
\end{array}\right)\left(\begin{array}{l}
u_{a} \\
u_{b}
\end{array}\right)
$$

Equation 34 shows that as the mass of a particle tends to zero then $\gamma_{5}$ tends to the helicity operator. In the Standard Model the neutrino is assumed to be massless and so the neutrino's helicity is the same as it's chirality. Left-handed chirality state massive particles will have 
mainly left-handed helicity but with some right-handed helicity and vice versa for right-handed chirality state massive particles. Helicity projection operators may be introduced:

$$
P_{L} \equiv\left(\frac{1-\gamma_{5}}{2}\right) \quad, \quad P_{R} \equiv\left(\frac{1+\gamma_{5}}{2}\right)
$$

satisfying:

$$
P_{R}^{2}=P_{R} \quad, \quad P_{L}^{2}=P_{L} \quad, \quad P_{R} P_{L}=P_{L} P_{R}=0 \quad, \quad P_{R}+P_{L}=1
$$

and then the left-handed and right-handed helicity components of Dirac spinors may by defined as:

$$
u_{L} \equiv P_{L} u \quad, \quad u_{R} \equiv P_{R} u
$$

These relations allow us to write the V-A current between to fermionic Dirac spinors as:

$$
\begin{aligned}
\bar{u}_{1} \gamma^{\mu} \frac{1-\gamma_{5}}{2} u_{2} & =\bar{u}_{1} \gamma^{\mu} P_{L} u_{2}=\bar{u}_{1} \gamma^{\mu} P_{L}^{2} u_{2} \\
& =\bar{u}_{1} \gamma^{\mu} P_{L} u_{2 L}=\bar{u}_{1} P_{R} \gamma^{\mu} u_{2 L} \\
& =u_{1}^{\dagger} P_{L} \beta \gamma^{\mu} u_{2 L}=u_{1 L} \gamma^{\mu} u_{2 L}
\end{aligned}
$$

Equation 38 shows that the V-A structure, that has been built into the theory of the weak interaction, implies that only the chiral L components of fermions enter into weak interactions (a similar argument shows that only the chiral $\mathrm{R}$ components of anti-fermions enter into weak interactions). Furthermore, due to the helicity operator transforming as a pseudoscalar under parity, it can be shown that the V-A structure of the weak interaction also implies that the positive helictity components of all massive fermions are suppressed in interactions by factors of order $m / E$ (and vice versa for the negative helicity components of anti-fermions).

The Standard Model does not predict the helicity state of the neutrino but, as it was assumed to be massless, the neutrino could have either fully positive or negative helicity. In 1958 Gold- 
haber et al [16] considered the capture of electrons on ${ }^{152} \mathrm{Eu}$ and showed that the helicity of the emitted neutrinos was (within errors) $100 \%$ negative. This result was taken as strong confirmation of the V-A nature of the weak interaction and strong motivation for massless neutrinos in the Standard Model.

The V-A theory was very successful but it still assumed a current-current interaction which was accompanied by a number of theoretical issues. In the 1960s physicists worked towards a gauge theory of the weak interaction that involved the introduction of a weak gauge symmetry group and resulting intermediate vector boson fields (introduced to keep the Lagrangian invariant under certain local transformations). The weak interaction was unified with the electromagnetic interaction and, through spontaneous symmetry breaking and the Higgs mechanism, these intermediate particles aquired masses and became the familiar photon, $Z^{0}$ and $W^{ \pm}$bosons of the Standard Model.

\subsubsection{The Glashow-Weinberg-Salam Electroweak Gauge Theory}

The theory of weak interactions views $e_{L}^{-}$and $v_{e L}$ as two states of the same particle under charged current (CC) processes which suggests that this pair (along with $\mu_{L}^{-} \leftrightarrow v_{\mu L}, u_{L} \leftrightarrow d_{L} \ldots$ ) transform as doublets under some symmetry group. The group, originally proposed in 1961 by Glashow [17] and the worked with in 1967 by Weinberg [18] and in 1968 by Salam [19]), was $S U(2)$.

The group is labelled $S U(2)_{L}$ to denote the fact that only the left-handed chiral components of the fields enter the weak interaction and corresponds to transformations in the internal space of weak isospin. $S U(2)$ is isomorphic to $S O(3)$ and so such tranformations can be thought of as rotations in a three-dimensional weak isospin-space. We use $I$ and $I_{3}$ to denote the quantum numbers of weak isospin and make the following assignments for the leptonic fields:

$$
I=\frac{1}{2} \quad\left\{\begin{array}{l}
I_{3}=+1 / 2 \\
I_{3}=-1 / 2
\end{array} \quad\left(\begin{array}{c}
\hat{\mathrm{v}}_{e} \\
\hat{e}^{-}
\end{array}\right)_{L}\left(\begin{array}{c}
\hat{\mathrm{v}}_{\mu} \\
\hat{\mu}^{-}
\end{array}\right)_{L}\left(\begin{array}{c}
\hat{\mathrm{v}}_{\tau} \\
\hat{\tau}^{-}
\end{array}\right)_{L}\right.
$$

The transformations can be written as: 


$$
\left(\begin{array}{c}
\hat{\mathrm{v}}_{e} \\
\hat{e}^{-}
\end{array}\right)_{L}^{\prime}=\exp (-i \boldsymbol{\alpha} \cdot \boldsymbol{\tau} / 2)\left(\begin{array}{c}
\hat{\mathrm{v}}_{e} \\
\hat{e}^{-}
\end{array}\right)_{L}
$$

where $\tau$ are the Pauli matrices (denoted such to show that they act in an internal space). This group could be considered as a local gauge invariance (meaning local transformations, $\boldsymbol{\alpha}\left(x^{\mu}\right)$, should be allowed but not change the observed physics) and would entail the introduction of three gauge fields (for local definitions of the three axes in weak isospin-space); two of the fields would have 'charge' \pm 1 (to allow transitions between the doublet members) with the third being a neutral field. Weak neutral current (NC) interactions were first reported in 1973 by Hasert et al [20] using the Gargamelle bubble chamber experiment at CERN and are known not to be pure V-A in nature. As such this third potential gauge field, coming from the pure V-A phenomenology, would not suitably describe NC interactions.

The solution was the unification of the weak and electromagnetic interactions via the addition of an extra $U(1)$ gauge group resulting in an $S U(2)_{L} \otimes U(1)$ structure. This new gauge group had to include an entity corresponding to a right-handed electron (for the electromagnetic interaction) and, because such an object is not observed in CC weak interactions, this entity had to be a singlet in weak isospin-space. For example, the first generation of leptons and quarks is arranged into $S U(2)$ doublets:

$$
\left(\begin{array}{c}
\hat{v}_{e} \\
\hat{e}^{-}
\end{array}\right)_{L}\left(\begin{array}{l}
\hat{u} \\
\hat{d}
\end{array}\right)_{L}
$$

and $S U(2)$ singlets; $\hat{e}_{R}^{-}, \hat{u}_{R}$ and $\hat{d}_{R}$. Then the weak hypercharge, $Y$, was introduced (to differentiate between left-handed doublet and right-handed singlet particles) in analogy with the 'ordinary' charge of the electromagentic interaction where:

$$
Y=2 Q-2 I_{3}
$$

where $Q$ is the electric charge and $I_{3}$ the third component of weak isospin. Local transformations in the internal space of weak isospin and local phase changes, corresponding to the $U(1)$ 
part of the electroweak symmetry group, should not affect the observed physics and can be expressed as:

$$
\left.\hat{\chi}_{L}^{\prime}=\exp \left(i g_{w} \boldsymbol{\alpha}\left(x^{\mu}\right) \cdot \boldsymbol{\tau}\right) / 2+i g^{\prime} \beta\left(x^{\mu}\right) Y\right) \hat{\chi}_{L}
$$

where $\hat{\chi}_{L}$ is a left-handed chiral doublet, $g_{w}$ and $g^{\prime}$ are coupling constants and $x^{\mu}$ is a point in space-time. In order to keep the Lagrangian invariant under such local $S U(2) \otimes U(1)$ transformations four new gauge fields must be introduced; two charged $\left(W_{1,2}^{\mu}\right)$ and one neutral field $\left(W_{3}^{\mu}\right)$ for the $S U(2)$ part of the symmetry group and a second neutral field $\left(B^{\mu}\right)$ for the $U(1)$ part of the symmetry group. Again the charged fields are responsible for raising and lowering members of the left handed chiral doublets whilst the two neutral fields will between them account for both weak NC and electromagnetic interactions. The gauge invariant Lagrangian can be written as:

$$
\begin{aligned}
\overline{\hat{\chi}}_{L} \gamma^{\mu}\left(i \partial_{\mu}-g_{w} \frac{\tau}{2} \cdot \hat{\mathbf{W}}_{\mu}-\frac{g^{\prime}}{2} Y \hat{B}_{\mu}\right) \hat{\chi}_{L} & +\overline{\hat{\chi}}_{R} \gamma^{\mu}\left(i \partial_{\mu}-\frac{g^{\prime}}{2} Y \hat{B}_{\mu}\right) \hat{\chi}_{R} \\
& -\frac{1}{4} \hat{W}_{\mu v} \hat{W}^{\mu v}-\frac{1}{4} \hat{B}_{\mu v} \hat{B}^{\mu v}
\end{aligned}
$$

where the final two terms are the self-interactions of the introduced gauge fields and the righthanded chiral fields only interact via the $\hat{B}^{\mu}$ leaving electromagnetism free from $\gamma_{5}$ and parity violating terms.

The electroweak Lagrangian of equation 44 is not permitted to contain mass terms for the gauge fields, such as $m^{2} B_{\mu} B^{\mu}$. However, through spontanteous symmetry breaking the massless $\hat{W}_{1}^{\mu}$ and $\hat{W}_{2}^{\mu}$ combine to form the massive $\hat{W}^{ \pm}$fields and the massless $\hat{W}_{3}^{\mu}$ and $\hat{B}^{\mu}$ combine to form the massive $\hat{Z}^{0}$ and massless photon fields. These fields are viewed as propagators (exchanged virtual bosons) and can be included in the construction of Feynamn diagrams for Standard Model weak processes such as shown in figure 7.

The matrix element for the transition shown in figure 7 can now be written, in terms of Dirac spinors and including the exchanged virtual boson, as: 


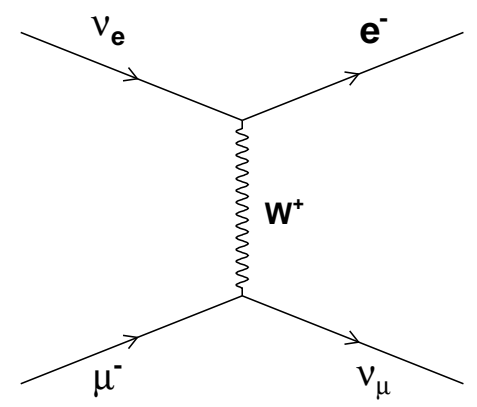

Figure 7: $W^{+}$exchange process for $\mathrm{v}_{e}+\mu^{-} \rightarrow e^{-}+v_{\mu}$.

$$
\left[-\frac{i g_{w}}{\sqrt{2}} \bar{u}_{v_{\mu}} \frac{1}{2} \gamma^{\mu}\left(1-\gamma_{5}\right) u_{\mu^{-}}\right]\left[i \frac{-g^{\mu v}+q^{\mu} q^{v} / M_{W}^{2}}{q^{2}-M_{W}^{2}}\right]\left[-\frac{i g_{w}}{\sqrt{2}} \bar{u}_{e^{-}} \frac{1}{2} \gamma_{\mu}\left(1-\gamma_{5}\right) u_{v_{e^{-}}}\right]
$$

where $g_{w} / \sqrt{2}$ is the coupling strength at each vertex, $g^{v \mu}$ is the metric tensor and $q^{2}$ the squared four-momentum transfer between the vertices.

At low $q^{2}\left(q^{2} \ll M_{W}^{2}\right)$ the W-propagator can be replaced by the constant term $g^{\mu v} / M_{W}^{2}$ leading to a matrix element, as shown in equation 46 , that looks very similar to the current-current form. In fact the V-A current-current interaction can be thought of as the low energy approximation of the full Glashow-Weinberg-Salam gauge theory of electroweak interactions.

$$
-\frac{i g_{w}^{2}}{8 M_{W}^{2}} \bar{u}_{v_{\mu}} \frac{1}{2} \gamma^{\mu}\left(1-\gamma_{5}\right) u_{\mu^{-}} \bar{u}_{e^{-}} \frac{1}{2} \gamma^{\mu}\left(1-\gamma_{5}\right) u_{v_{e^{-}}}
$$

where it can be seen that $g_{w}$ relates to Fermi's constant via:

$$
\frac{G_{F}}{\sqrt{2}}=\frac{g_{w}^{2}}{8 M_{W}^{2}}
$$

Equation 46 illustrates how the transition amplitudes for simple leptonic scattering in the Standard Model can be built from V-A currents involving Dirac spinors, a virtual boson propagator and interaction coupling strengths. The weak interaction is known as 'universal' because all lepton pairs undergo the same form of the V-A coupling, with the same 'strength parameter', 
and this property means that cross sections for a large number of scattering processes can be computed using the above formalism. However, the phenomenology of neutrino scattering off nucleons, and nucleons that are bound in nuclei, faces some additional complications.

\subsection{Neutrino-Nucleus Scattering}

\subsubsection{Neutrino-Nucleon Scattering}

The pure V-A structure of weak interactions, being the equal difference of the vector and axial parts, is also valid at weak vertices where we have a current between quarks such as:

$$
\bar{u}_{u} \gamma_{\mu}\left(1-\gamma_{5}\right) u_{d}
$$

but when moving from the quark level to vertices including nucleons there are additional strong interaction effects to consider. The proton and neutron are not simple pointlike particles but have some internal structure with quantum chromodynamics (QCD) allowing many processes such as the emission of gluons from valence quarks and quark-antiquark pair formation from the gluons. It is known that, even with such processes occuring, the net electric charge is conserved (the proton always has a charge of $e$ ) but there is no reason to believe that the same is true in the weak interaction. For example, when a quark-antiquark pair is formed the net contribution of this pair to the weak interaction may not be zero.

If intermediate vector boson and $q^{2}$ considerations are, for the moment, put aside then the effects of the strong interactions can be accounted for by making the following replacement in the weak current:

$$
\left(1-\gamma_{5}\right) \rightarrow\left(c_{V}-c_{A} \gamma_{5}\right)
$$

where the correction factors $c_{V}$ and $c_{A}$ can be determined experimentally. Experiments, such as those looking at neutron $\beta$-decay, have measured these constants to be:

$$
c_{V}=1.000 \pm 0.003 \quad, \quad c_{A}=1.26 \pm 0.02
$$


Equation 50 shows that the vector part of the weak current is not modified by the presence of strong interations in the nucleon. This suggests that there is some conservation law that 'protects' the vector current in the same way that the electromagnetic charge is protected. This conservation law is known as the Conserved Vector Current (CVC) hypothesis and was theoretically formulated in 1958 by Feynman and Gell-Mann [21]. They postulated that the vector part of the weak current $\bar{u}_{p} \gamma_{\mu} u_{n}$, it's conjugate current $\bar{u}_{n} \gamma_{\mu} u_{p}$ and the electromagnetic current $\bar{u}_{p} \gamma_{\mu} u_{p}$ form a triplet of conserved currents in the internal isospin space of the strong interaction.

Equation 50 also shows that the axial part of the weak current is not heavily modified by the presence of the strong interaction and hinted at the Partially Conserved Axial Current (PCAC) hypothesis that was presented in a paper in 1960 by Gell-Mann and Levy [22].

\subsubsection{The Kinematics of Neutrino-Nucleon Scattering}

Before further discussing the theory of neutrino-nucleon scattering it is helpful to formally introduce the kinematic quantities associated with a general $\mathrm{CC}$ scattering process between a neutrino and a nucleon. Figure 8 shows the diagram for the process $v_{\mu}+P \rightarrow \mu^{-}+X$ (where $X$ denotes the hadronic system) and labels the measured (lab frame) quantites for such an event using the MINOS detectors and the centre of mass frame four-momenta of the involved particles.

Table 2 lists the Lorentz invariant kinematic quantities (quantities that take the same value independant of the frame of reference in which they are calculated) that describe the interaction and presents the formulae for constructing these variables using both the centre of mass frame particle four-momenta (as labelled in figure 8) and using the lab-frame measured quantities.

In a quasi-elastic scattering (QEL) event, $\mathrm{v}_{\mu}+n \rightarrow \mu^{-}+p$, the neutrino is considered to scatter off an entire nucleon, rather than it's constituent partons, and the target nucleus is modified but does not break up. If an event is assumed to be a quasi-elastic interaction then the hadronic system constitutes a single proton $\left(W^{2}=M_{\text {proton }}^{2}\right)$. 


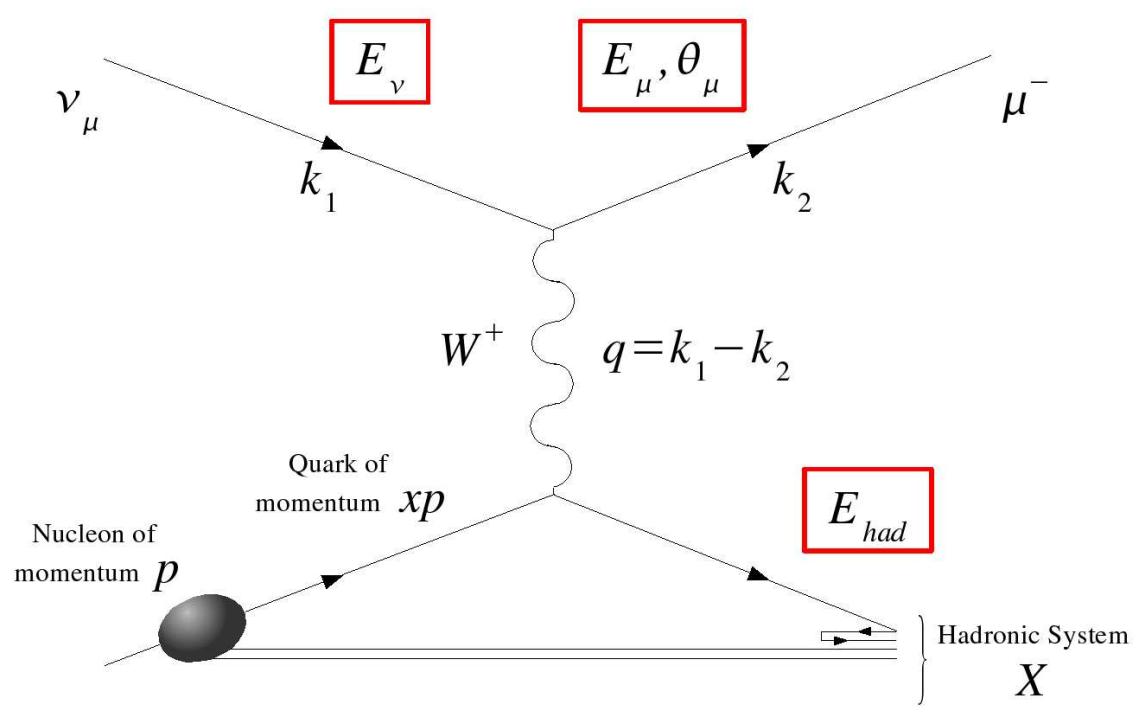

Figure 8: Kinematics of $C C \mathrm{v}_{\mu}-N$ scattering. The diagram labels the four-momenta of the involved particles in the centre of mass frame and the variables contained inside the red boxes correspond to the lab frame quantities measured by the MINOS detectors. The Bjorken scaling variable, $x$, denotes the fraction of the nucleon momentum carried by the struck quark.

\begin{tabular}{|c|c|c|}
\hline Lorentz Invariant & $\begin{array}{c}\text { Centre of Mass } \\
\text { Frame Calculation }\end{array}$ & $\begin{array}{c}\text { Lab Frame } \\
\text { Calculation }\end{array}$ \\
\hline \hline $\begin{array}{c}\text { Energy transferred to } \\
\text { the hadronic system; } v\end{array}$ & $\boldsymbol{p} \cdot \boldsymbol{q} / M$ & $E_{\text {had }}$ \\
\hline Inelasticity; $y$ & $(\boldsymbol{p} \cdot \boldsymbol{q}) /\left(\boldsymbol{p} \cdot \boldsymbol{k}_{1}\right)$ & $E_{\text {had }} / E_{\mathrm{v}}$ \\
\hline $\begin{array}{c}\text { Squared four-momentum } \\
\text { transfer; } Q^{2}\end{array}$ & $-\boldsymbol{q}^{2}$ & $2 E_{\mathrm{v}} E_{\mu}\left(1-\cos \left(\theta_{\mu}\right)\right)$ \\
\hline Bjorken scaling variable; $x$ & $Q^{2} / 2 \boldsymbol{p} \cdot \boldsymbol{q}$ & $Q^{2} / 2 E_{\text {had }} M$ \\
\hline $\begin{array}{c}\text { Squared invariant mass of } \\
\text { of the hadronic system, } W^{2}\end{array}$ & $(\boldsymbol{p}+\boldsymbol{q})^{2}$ & $M^{2}+2 E_{\text {had }} M-Q^{2}$ \\
\hline
\end{tabular}

Table 2: Calculation of Lorentz invariant kinematic quantites describing charged current neutrinonucleon scattering. In the formulae $M$ is the mass of the stuck nucleon. 
A consequence of this assumption is that a number of the kinematic variables describing a quasi-elastic interaction can be redefined using just the measured quantities corresponding to the outgoing muon. These QEL-assumed kinematic quantities are introduced in the following list and both they and the standard kinematic variables of table 2 will be referred to extensively throughout this thesis.

- Neutrino energy: in MINOS the neutrino energy is usually reconstructed by summing the visible energy in the detector for a given event $\left(E_{v}=E_{\mu}+E_{\text {had }}\right)$ but if an event is assumed to be a quasi-elastic interaction then a QEL-assumed neutrino energy, $E_{\mathrm{v}}^{Q E L}$, can be constructed, using just the kinematics of the muon, according to:

$$
E_{\mathrm{v}}^{Q E L}=\frac{E_{\mu} M-\left(M_{\mu}^{2} / 2\right)}{M-E_{\mu}+p_{\mu} \cos \left(\theta_{\mu}\right)}
$$

where $M$ is the mass of the struck nucleon, $M_{\mu}$ is the mass of the outgoing muon and equation 51 neglects terms that are multiplied by the binding energy of the struck nucleon inside the target nucleus.

- Squared four-momentum transfer: the QEL-assumed four-momentum transfer between the leptonic and hadronic vertices, $Q_{Q E L}^{2}$, can also be computed using just the kinematics of the outgoing muon according to:

$$
Q_{Q E L}^{2}=-2 E_{v}^{Q E L}\left[E_{\mu}-p_{\mu} \cos \left(\theta_{\mu}\right)\right]+M_{\mu}^{2}
$$

\subsubsection{The Quasi-Elastic Cross Section}

For a QEL scattering event with four-momenta as given by equation 53:

$$
\mathrm{v}_{\mu}(p)+n(P) \rightarrow \mu^{-}\left(p^{\prime}\right)+p\left(P^{\prime}\right)
$$

we can write the most general matrix element from electroweak theory as: 


$$
\frac{G_{F}}{\sqrt{2}} \bar{u}_{\mu^{-}}\left(p^{\prime}\right) \gamma_{\mu}\left(1-\gamma_{5}\right) u_{v_{\mu}}(p) \cos \left(\theta_{C}\right) \bar{u}_{p}\left(P^{\prime}\right) \Gamma_{C C}^{\mu}\left(q^{2}\right) u_{n}(P)
$$

where $\theta_{C}$ is the Cabibbo angle (a measure of the probability that one flavor of quark will change into other flavors under the weak interaction) and $\Gamma_{C C}^{\mu}\left(q^{2}\right)$ is a term containing complex weak form factors for the nucleon:

$$
\begin{aligned}
\Gamma_{C C}^{\mu} & =\gamma^{\mu} F_{V}\left(q^{2}\right)+\frac{i \sigma^{\mu v} q^{v}}{2 M} F_{M}\left(q^{2}\right)+\frac{q_{\mu}}{M} F_{S}\left(q^{2}\right) \\
& +\left(\gamma^{\mu} F_{A}\left(q^{2}\right)+\frac{i \sigma^{\mu v} q^{v}}{2 M} F_{T}\left(q^{2}\right)+\frac{q_{\mu}}{M} F_{P}\left(q^{2}\right)\right) \gamma_{5}
\end{aligned}
$$

where $M$ is the mass of the struck nucleon. These weak form factors parameterise the amount of each type of weak current participating in the interaction. They are functions of the fourmomentum carried by the vector boson 'probe', $q^{2}$, reflecting the fact that for different values of $q^{2}$ the boson 'sees' different levels of the nucleon internal structure (and hence different amounts of each coupling type contribute) and are related in the $q^{2}=0$ limit to the correction factors $c_{V}$ and $c_{A}$ of equation 49 .

Using table 1 the V-type nucleon form factors can be identified as; the vector form factor $F_{V}$ (also written as $F_{V}^{1}$ ), the weak magnetism form factor $F_{M}$ (often written ${ }^{2}$ as $\xi F_{V}^{2}$ where $\xi=k_{p}-k_{n}+1$ and $k_{p / n}$ are the anomalous magnetic moments of the proton and neutron and $F_{V}^{2}$ is a second V-type form factor) and the scalar form factor $F_{S}$. The A-type form factors can be similarly identified as; the axial-vector form factor $F_{A}$, the pseudotensor form factor $F_{T}$ and the pseudoscalar form factor $F_{P}$.

These, in general complex, form factors must be measured experimentally and it has been confirmed that neither the scalar-type or pseudotensor-type currents contribute to the weak interaction with nucleons. This measurement has also been explained theoretically, for example

\footnotetext{
${ }^{2}$ Note that the superscript ' 2 ' in $F_{V}^{2}$ denotes that this is a second V-type form factor and should not be read as raising the form factor to the second power.
} 
in the 1972 paper of Llewellyn-Smith [23], where the preservation of time reversal invariance (T) requires all the form factors to be real and the preservation of charge symmetry (C) requires both $F_{S}$ and $F_{T}$ to be imaginary. As such these two form factors are set to zero:

$$
F_{S}\left(q^{2}\right)=F_{T}\left(q^{2}\right)=0 \quad \forall q^{2}
$$

In cross section calculations $F_{P}$ is multipled by $m_{\mu}^{2}$ and so for neutrino energies $E_{\mathrm{v}} \gg m_{\mu}^{2}$ the pseudoscalar form factor can be neglected ${ }^{3}$. Neglecting the pseudoscalar interaction and setting the scalar and pseudotensor form factors to zero, equation 55 can be reduced to:

$$
\Gamma_{C C}^{\mu}=\gamma^{\mu}\left[F_{V}^{1}\left(q^{2}\right)-\gamma_{5} F_{A}\left(q^{2}\right)\right]+\frac{i \sigma^{\mu v} q^{v}}{2 M} \xi F_{V}^{2}\left(q^{2}\right)
$$

Using this reduced form of the description of the hadronic vertex and turning the matrix element of equation 54 into a cross section, the differential cross section, with respect to $q^{2}$, for QEL scattering can then be expressed as in equation $58^{4}$ :

$$
\frac{d \sigma}{d\left|q^{2}\right|}=\frac{M^{2} G_{F}^{2} \cos ^{2}\left(\theta_{c}\right)}{8 \pi E_{v}^{2}}\left[A\left(q^{2}\right)-B\left(q^{2}\right) \frac{s-u}{M^{2}}+C\left(q^{2}\right) \frac{(s-u)^{2}}{M^{4}}\right]
$$

where $s-u=4 E_{\mathrm{v}} M+q^{2}-m_{l}^{2}, m_{l}$ is the mass of the produced charged lepton and the factors $A\left(q^{2}\right), B\left(q^{2}\right)$ and $C\left(q^{2}\right)$ can be written:

$$
\begin{aligned}
A\left(q^{2}\right) & =\frac{m_{l}^{2}-q^{2}}{4 M^{2}}\left[\left(4-\frac{q^{2}}{M^{2}}\right)\left|F_{A}\right|^{2}-\left(4+\frac{q^{2}}{M^{2}}\right)\left|F_{V}^{1}\right|^{2}\right. \\
& -\frac{q^{2}}{M^{2}}\left|\xi F_{V}^{2}\right|^{2}\left(1+\frac{q^{2}}{4 M^{2}}\right)-\frac{4 q^{2} R e F_{V}^{1} \xi F_{V}^{2}}{M^{2}} \\
& \left.-\frac{m_{l}^{2}}{M^{2}}\left(\left(F_{V}^{1}+\xi F_{V}^{2}\right)^{2}+\left|F_{A}\right|^{2}\right)\right]
\end{aligned}
$$

\footnotetext{
${ }^{3}$ If scattering involving the $\tau$ was considered $F_{P}$ should not be neglected.

${ }^{4}$ This is the equation for neutrinos $\left(v n \rightarrow l^{-} p\right)$. For anti-neutrinos $\left(\bar{v} p \rightarrow l^{+} n\right)$ the term $-B\left(q^{2}\right)$ must be replaced with $+B\left(q^{2}\right)$.
} 


$$
\begin{gathered}
B\left(q^{2}\right)=-\frac{q^{2}}{M^{2}}\left[\left(F_{V}^{1}+\xi F_{V}^{2}\right) R e F_{A}\right] \\
C\left(q^{2}\right)=\frac{1}{4}\left(\left|F_{A}\right|^{2}+\left|F_{V}^{1}\right|^{2}-\frac{q^{2}}{4 M^{2}}\left|\xi F_{V}^{2}\right|^{2}\right)
\end{gathered}
$$

To further constrain the vector form factors of equation 58 it is helpful to briefly consider the elastic scattering of electrons from nucleons. Since the 1950s it has been known that the proton (and neutron) can not just be a mathematical point charge with a pointlike magnetic moment and in 1950 Rosenbluth [25] was one of the first to consider separating these two parts and using form factors to describe their contributions. The ideas of Rosenbluth were applied to elastic electon-proton scattering and it can be shown, for example in [24], that the electromagnetic current part of the hadronic vertex for the elastic scattering of electrons off nucleons has the following general form:

$$
\bar{u}_{p, n}\left(P^{\prime}\right)\left[\gamma^{\mu} F_{p, n}^{1}\left(q^{2}\right)-\frac{\sigma^{\mu v} q^{v}}{2 M} F_{p, n}^{2}\left(q^{2}\right)\right] u_{n, p}(P)
$$

where $F_{p, n}^{1}$ is the Dirac form factor, which relates to the deviation of the nucleon from a point charge particle, and $F_{p, n}^{2}$ is the Pauli form factor, which relates to the deviation of the nucleon from a pointlike magnetic moment. It is interesting to note that whilst equations 57 and 62 are very similar there are no parity violating $\gamma_{5}$ terms present for the electromagnetic interaction.

The Dirac and Pauli form factors are often combined into the Sachs [26] electric and magnetic form factors for the proton and neutron according to:

$$
G_{E}^{p, n}\left(Q^{2}\right)=F_{p, n}^{1}\left(Q^{2}\right)-\frac{Q^{2}}{4 M^{2}} F_{p, n}^{2}\left(Q^{2}\right) \quad, \quad G_{M}^{p, n}\left(Q^{2}\right)=F_{p, n}^{1}\left(Q^{2}\right)+F_{p, n}^{2}\left(Q^{2}\right)
$$

The Sachs form factors have been well measured in electron scattering experiments and furthermore these measurements have established a common form that describes all of the form factors; the dipole form factor $G_{D}\left(q^{2}\right)$ : 


$$
\begin{array}{cl}
G_{E}^{p}\left(q^{2}\right)=G_{D}\left(q^{2}\right) \quad & G_{E}^{n}\left(q^{2}\right)=0 \\
G_{M}^{p}\left(q^{2}\right)=\mu_{p} G_{D}\left(q^{2}\right) \quad, \quad & G_{M}^{n}\left(q^{2}\right)=\mu_{n} G_{D}\left(q^{2}\right)
\end{array}
$$

where:

$$
G_{D}\left(q^{2}\right)=\frac{1}{\left(1-\frac{q^{2}}{M_{V}^{2}}\right)^{2}}
$$

and $M_{V}$ is the vector mass (which has also been well measured in electron scattering experiments). It should be noted that $G_{E}^{n}\left(q^{2}=0\right)$ has to be zero because the neutron has no net electric charge and this form factor is usually set to zero for all values of $q^{2}$.

Assuming the CVC hypothesis the Sachs form factors (which derived from the nucleon form factors for the electromagnetic part of the elastic scattering of electrons off nucleons as given in equation 62) can be related to the weak nucleon vector form factors of equation 57 according to:

$$
\begin{gathered}
F_{V}^{1}\left(q^{2}\right)=\frac{\left[G_{E}^{p}\left(q^{2}\right)-G_{E}^{n}\left(q^{2}\right)\right]-\frac{q^{2}}{4 M^{2}}\left[G_{M}^{p}\left(q^{2}\right)-G_{M}^{n}\left(q^{2}\right)\right]}{1-\frac{q^{2}}{4 M^{2}}} \\
F_{V}^{2}\left(q^{2}\right)=\frac{\left[G_{M}^{p}\left(q^{2}\right)-G_{M}^{n}\left(q^{2}\right)\right]-\left[G_{E}^{p}\left(q^{2}\right)-G_{E}^{n}\left(q^{2}\right)\right]}{1-\frac{q^{2}}{4 M^{2}}}
\end{gathered}
$$

Equations 66 and 67 show that the weak vector form factors that enter into the calculation of the cross section for QEL scattering can be constrained by the accurately measured Sachs form factors using the CVC hypothesis. In the above prescription (where the Sachs form factors are expressed in terms of the dipole form factor) the weak vector form factors are known as 'Dipole Form Factors'.

The only remaining uncertainty in the cross section (equation 58) for QEL scattering comes 
from the axial-vector form factor, $F_{A}\left(q^{2}\right)$. By analogy with the vector case the axial-vector form factor can be written using a dipole approximation as follows:

$$
F_{A}\left(q^{2}\right)=\frac{F_{A}(0)}{\left(1-\frac{q^{2}}{\left(M_{A}^{O E L}\right)^{2}}\right)^{2}}
$$

where $M_{A}^{Q E L}$ is the axial-vector mass. $F_{A}\left(q^{2}=0\right)$ has been measured in neutron $\beta$-decay experiments (following the discussion of the corrections factors $c_{V}$ and $c_{A}$ ) but the $q^{2}$ dependance of the axial form factor (which is equivalent to measuring $M_{A}^{Q E L}$ ) needs to be extracted from weak CC neutrino-nucleon QEL scattering data. The dipole form is quite similar to the form for a propagated vector boson and the axial-vector mass could be thought of as the mass of the propagated boson corresponding to the axial-vector part of the weak interaction.

Figures 9 and 10 show the differential cross section for CC QEL scattering as a function of $Q^{2}$ for mono-energetic neutrinos scattering off free nucleons using different values of $M_{A}^{Q E L}$. In

the first figure the curves are normalised to area and show that changing the value of $M_{\AA}^{Q E L}$ has an effect on the shape of the cross section whilst in the second figure the curves are absolutely normalised and show that changes to $M_{A}^{Q E L}$ also influence the overall normalisation of the cross section.

\subsubsection{Nuclear Effects}

For values of $Q^{2} \simeq 0.2 \mathrm{GeV}^{2}$ the vector boson probe has a wavelength that is approximately the size of the nuclear diameter for iron (the main nuclei with which neutrinos interact in the MINOS detectors) and so, for $Q^{2}$ values below $\sim 0.2 \mathrm{GeV}^{2}$, the probe does not see the internal structure of an individual nucleon but rather the scattering process is affected by the fact that the target nucleon is embedded in a nucleus. The type and level of nuclear effects are dependant on the target nucleus in question and, for example, older experiments that considered QEL neutrino-deuterium scattering are less sensitive to these effects than MINOS where the neutrinos are primarily incident on heavier iron nuclei. 




Figure 9: Effect of changing $M_{A}^{Q E L}$ upon the free nucleon $Q E L$ scattering crosss section. The curves correspond to mono-energetic neutrinos (at $1 \mathrm{GeV}$ ) and do not include any nuclear effects. The cutoff at higher $Q^{2}$ is purely kinematic and the curves are normalised by area. Figure courtesy of [27].



Figure 10: Effect of changing $M_{A}^{Q E L}$ upon the free nucleon $Q E L$ scattering crosss section. The curves correspond to mono-energetic neutrinos (at $1 \mathrm{GeV}$ ) and do not include any nuclear effects. The cutoff at higher $Q^{2}$ is purely kinematic and the curves are absolutely normalised. Figure courtesy of [27]. 
Nuclear effects will change both $\sigma_{Q E L}$ and the kinematics of the final state. They include the Fermi motion of the struck nucleon (the movement of the nucleon inside the target nucleus), it's binding energy in the nucleus, Pauli blocking (a consequence of the application of the Pauli Exclusion Principle to the nucleus) and final state interactions (FSIs) such as intranuclear re-scattering and the Coulomb interaction with the nuclear remnant.

Pauli blocking is a consequence of the fact that identical fermions cannot occupy the same quantum state. In the case of QEL neutrino scattering this means that the struck nucleus can only be excited if there is an unoccupied final energy state for the outgoing nucleon. Recent experiments have used mean-field (MF) models such as the Hartree-Fock, shell and Fermi Gas models to describe Pauli blocking (and nucleon Fermi motion) for QEL scattering. In MF models the excitation of the nuclear system is described as the transition of a nucleon from a state below the Fermi surface (a certain value of nucleon momentum, the Fermi momentum, below which all energy levels are filled) to one above the Fermi surface. The MF models represent the nucleus as a translationally invariant system composed of an infinite number of nucleons with a momentum distribution given by:

$$
n(|\mathbf{p}|)=\frac{\tau}{\frac{4}{3} \pi k_{F}^{3}} \Theta\left(k_{F}-|\mathbf{p}|\right)
$$

where $\Theta$ is the Heaviside function, $\tau=Z$ or $N$ for the nucleus in question, $k_{F}$ is the Fermi momentum and $\mathbf{p}$ is the nucleon 3-momentum. The value of the Fermi momentum depends upon the nucleus in question but is typically 200-300 MeV. In Fermi Gas (FG) simulations all energy levels up to the Fermi surface are considered to be filled and any interaction with a momentum transfer that leaves the final state nucleon with a momentum less than the Fermi momentum is considered to be Pauli blocked.

QEL neutrino-nucleus scattering was first evaluated in a FG model in 1972 by Smith and Moniz [28] and can have large effects at low values of $Q^{2}$. Figure 11 shows an example of the effect of moving from a free nucleon description of $\sigma_{Q E L}$ to a FG model with 3 different values of the Fermi momentum. 


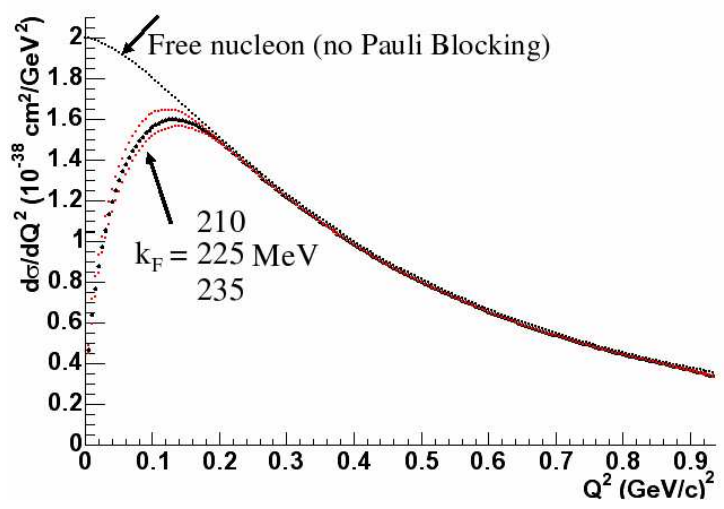

Figure 11: Implications of nuclear effects in a FG model, with 3 different values of the Fermi momentum, on the QEL cross section as a function of $Q^{2}$ compared to the free nucleon prediction. This figure was taken from a talk by M. Sakuda at the NuFact 05 conference.

The FG model improves agreement with experimental data, especially when combined with a model for FSIs, but it is not perfect in the $Q^{2}<0.2 \mathrm{GeV}^{2}$ region and much progress has been made in the last decade to improve understanding of nuclear effects beyond the MF approximation. Of particular interest is the development of nuclear spectral functions which include a better description of the momentum distribution of nucleons within nuclei [29].

Pauli blocking is the most prominent nuclear effect for studies of QEL scattering at low neutrino energies and low $Q^{2}$ but final state interactions are also important. FSIs deal with the passage of the final state through the nucleus and in the case of a $v_{\mu}$-CC QEL interaction the final state proton can be re-absorbed or re-scattered by the nuclear remnant leading to different observable particles and event kinematics in a detector. The propagation of the final state is usually handled, in current simulations, by an intranuclear cascade model such as presented in [30]. The ramifications of intranuclear re-scattering for MINOS are discussed further in [31].

\subsubsection{Deviations from the Dipole Form}

The amount of experimental data on the electromagnetic form factors of the proton and neutron has increased dramatically since the proposition of the dipole form approximation. In 2003 new fits were performed [32] to electron scattering data using an inverse polynomial form for each of the Sachs form factors: 


$$
G_{E, M}^{N}\left(Q^{2}\right)=\frac{G_{E, M}^{N}\left(Q^{2}=0\right)}{1+a_{2} Q^{2}+a_{4} Q^{4}+a_{6} Q^{6}+\cdots}
$$

$G_{E}^{n}$ is the Sachs electric form factor for the neutron and must take the value of zero at $q^{2}=0$ because the neutron has no net electric charge. Many neutrino experiments had assumed that this form factor was zero for all values of $q^{2}$ but the new 'BBA-2003 Form Factors' allowed $G_{E}^{n}$ to evolve away from zero with $q^{2}$. The change from using Dipole Form Factors to the BBA-2003 Form Factors has a reasonably large effect on the QEL cross section and figure 12 shows that the cross section is reduced by up to $\sim 7 \%$.

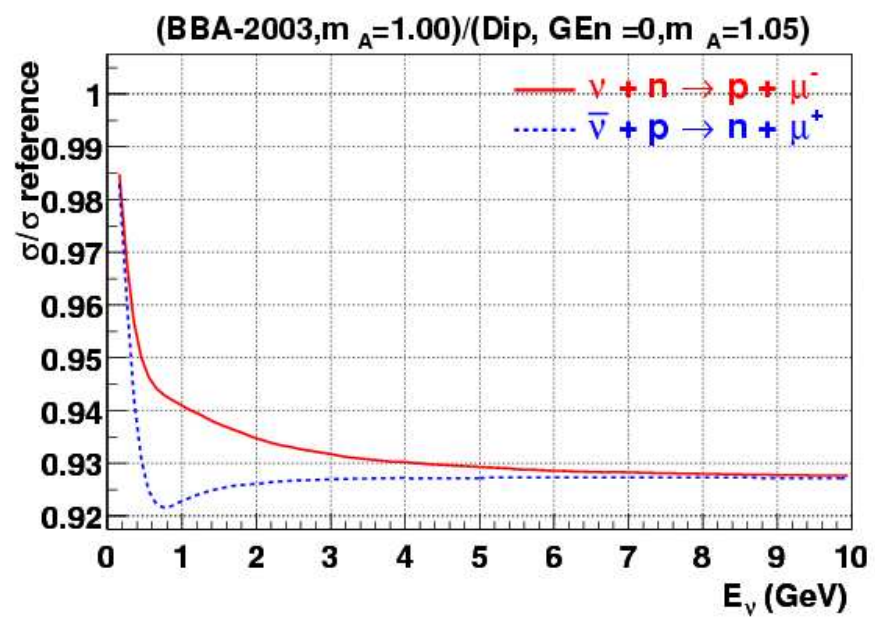

Figure 12: Ratios of $\sigma_{Q E L}$ versus energy using the BBA-2003 Form Factors with $M_{A}^{Q E L}=1.00 \mathrm{GeV}$ versus the Dipole Form Factors with $M_{A}^{Q E L}=1.05 \mathrm{GeV}$. The $M_{A}^{Q E L}$ values are chosen such that the sample $Q^{2}$ distributions are as similar as possible. Figure taken from [32].

Figures 13 are again taken from [32] and show the cross sections for QEL scattering on Carbon using the BBA-2003 Form Factors and $M_{A}^{Q E L}=1.0 \mathrm{GeV}$ for free nucleon and FG calculations along with experimental data taken using a number of different nuclear targets. They illustrate, in the case of neutrinos, that the shape of the QEL cross section is approximately flat with energy (above $\sim 1 \mathrm{GeV}$ ) and relatively well constrained whereas the overall normalisation of the cross section is not so well known.

In 2005 the BBA-2003 Form Factors were revised [33] using a new fitting function for the 

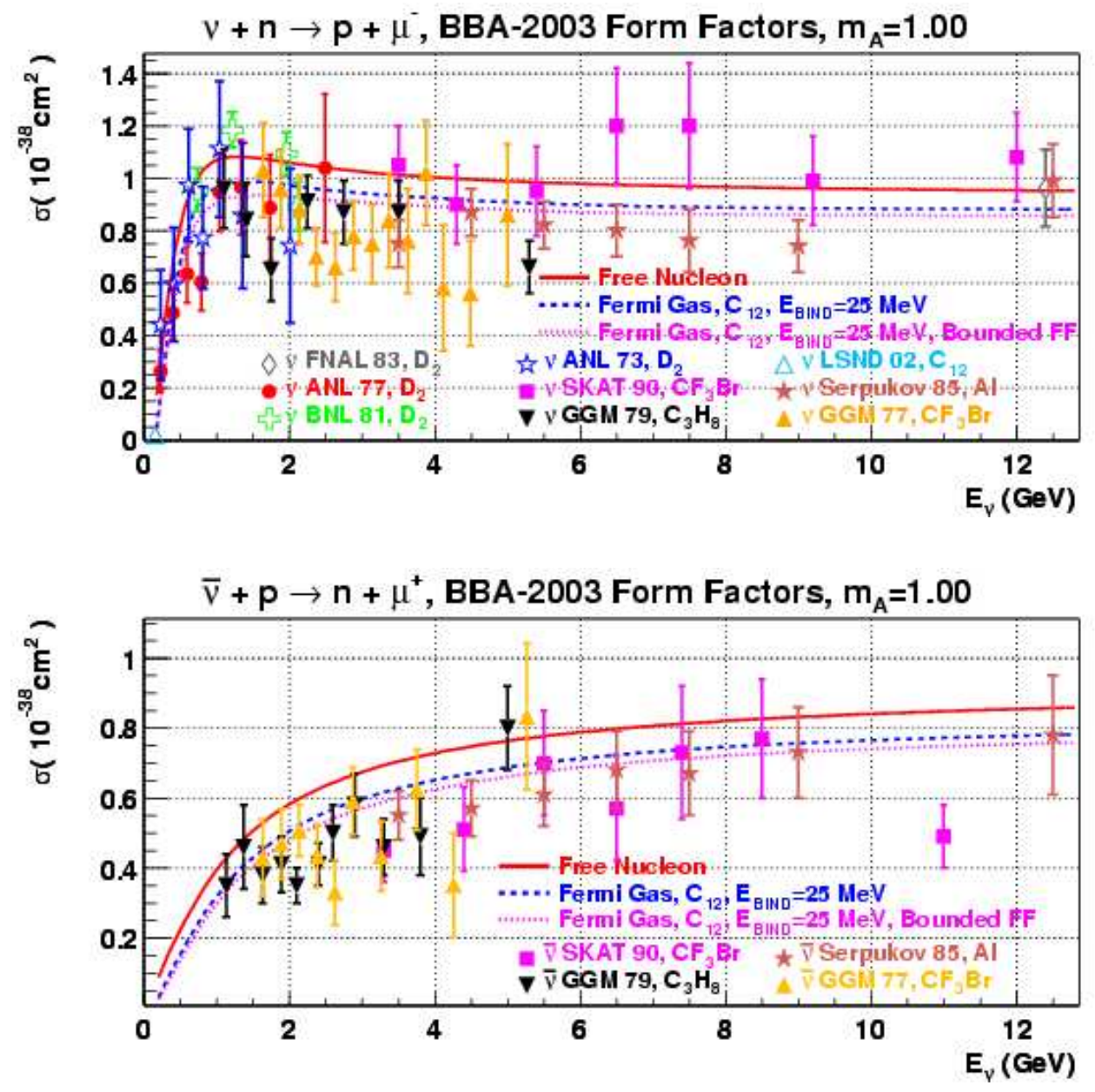

Figure 13: Cross sections for $v_{\mu}$-CC QEL and $\bar{v}_{\mu}$-CC QEL scattering along with data from various experiments. The calculations use $M_{A}^{Q E L}=1.0 \mathrm{GeV}, F_{A}(0)=-1.267, M_{V}^{2}=0.71 \mathrm{GeV}^{2}$ and BBA-2003 vector form factors. These figures were taken from [32]. 
nucleon electromagentic form factors and with the addition of some additional constraints. The resulting QEL scattering cross sections did not change significantly from those presented in [32] but, to differentiate this parameterisation from earlier work, these form factors are known as the 'BBA-2005 Form Factors'.

It is also worth restating that the dipole approximation for the axial-vector form factor, $F_{A}$, is not necessarily accurate and furthermore that it could depend upon the nuclear environment. As such, fixed target neutrino scattering experiments that use different nuclear targets could expect to measure slightly different values of $M_{A}^{Q E L}$ (within the dipole approximation) due to the different underlying parameterisations of $F_{A}$ required.

The next chapter of this thesis will introduce some of the previous measurements of $M_{\AA}^{Q E L}$, present a compilation of previous results and discuss some of the complications inherent in comparing the measurements made with various experiments stemming from sources such as differences in the target nuclei. 


\section{Measuring $M_{A}^{Q E L}$}

Electron scattering experiments, such as those at the Jefferson Laboratory in Virginia, have measured the vector form factors of the nucleons and neutron $\beta$-decay experiments have measured the value of $F_{A}\left(q^{2}=0\right)$ with both sets of measurements achieving high precision. However, only neutrino scattering experiments can extract the $q^{2}$ evolution of the axial-vector form factor and so, assuming a dipole form for $F_{A}$, measurements of the QEL cross section are essentially measurements of the axial-vector mass, $M_{\AA}^{Q E L}$.

There are a large number of previous experiments that have extracted $M_{A}^{Q E L}$ for a variety of nuclear targets and neutrino energies and with several different methods. This chapter will first present a brief introduction to the methodology of extracting $M_{A}^{Q E L}$ from QEL neutrinonucleus scattering data. Older results from a deuterium-filled bubble chamber will be discussed followed by results from more recent experiments using carbon and oxygen targets. The final section will collate results from a larger number of experiments and present the current worldaverage values.

\subsection{General Method for Extracting $M_{A}^{Q E L}$}

The only uncertainty in the differential cross section for quasi-elastic scattering as a function of $Q^{2}$ (as given by equation 58), when the measurements mentioned above are taken into account, is the axial-vector form factor $F_{A}\left(Q^{2}\right)$. In turn the only uncertainty in the dipole approximation for $F_{A}$ is the axial-vector mass, $M_{A}^{Q E L}$. Consequently neutrino scattering experiments extract $M_{A}^{Q E L}$ by analysing the $Q^{2}$ distribution for weak charged current QEL events.

Figures 9 and 10 from the previous chapter showed that changes to the value of $M_{A}^{Q E L}$ will affect both the shape and normalisation of the $Q^{2}$ distribution for QEL events and so experiments can consider using pure rate information, pure shape information or both. In figure 9 the curves were normalised by area illustrating the features that a shape-only fit uses to differentiate between values of $M_{A}^{Q E L}$ whilst in figure 10 the curves were absolutely normalised and illustrated the extra information that can be used in fits that look at both the shape and normalisation of the $Q^{2}$ distribution for QEL events. 


\subsection{Results from the Argonne 12-Foot Bubble Chamber}

Some of the most accurate previous measurements of $M_{A}^{Q E L}$ came from the 12-foot bubble chamber at the Argonne National Laboratory which was exposed to a neutrino beam produced at the Zero Gradient Synchrotron (ZGS) facility. The ZGS brought $12.4 \mathrm{GeV}$ protons to focus on a beryllium target with the produced charged hadrons focussed by two ${ }^{5}$ magentic horns. The hadrons, mainly $\pi^{-}$, were allowed to decay in a 30m long drift space with a steel and lead shield aborbing the resulting hadrons and charged leptons. The muon neutrinos passed through the shielding and this beam was incident on the deuterium filled bubble chamber.

The neutrino beam flux peaked at $\sim 0.5 \mathrm{GeV}$ with a tail out to $6 \mathrm{GeV}$ and was modelled using a simulation of the neutrino beamline and utilizing the measured yields of hadrons from $p-B e$ collisions. The uncertainty on the flux was estimated to be $\pm 15 \%$ except at the higher neutrino energies where the fraction of neutrinos coming from kaon decay increases and the lack of $K^{+}$ production measurements drove the uncertainty to $\pm 25 \%$.

The bubble chamber was filled with liquid deuterium heated to just below it's boiling point. As particles entered the chamber the pressure was decreased and the deuterium entered a superheated phase. Charged particles in the chamber then left ionization tracks around which the deuterium vaporised forming tiny bubbles. These bubbles grew in size as the chamber was expanded until the point where they could be photographed by an array of cameras. The bubble density around a track (or 'prong') was proportional to the particle energy loss and, since a magnetic field was applied to the chamber, the tracks were helical and allowed for a momentum measurement. The final analysis used a data sample consisting of $2.4 \times 10^{6}$ pictures of the chamber.

The bubble chamber pictures were scanned by physicists and the interesting one-, two- and three-prong events were recorded. The overall efficiency of the scanning process was estimated to be $(98 \pm 2) \%$ for events within the fiducial volume. In a deuterium filled bubble chamber

\footnotetext{
${ }^{5}$ The experiment originally ran with just one horn but a second was later added.
} 
the spectator proton, denoted $p_{s}$, from the QEL scattering process $v d \rightarrow \mu^{-} p p_{s}$ is sometimes visible leading to a three-prong event. All two- and three-prong events underwent geometric reconstruction and kinematic fitting to the QEL hypothesis ${ }^{6}$ and the final background level in the QEL sample was estimated to be $(2 \pm 2) \%$. The final analysis paper [34] used a data sample of 1737 events and, although this is not a large statistics sample, the extremely high QEL purity allowed for a precision measurement of $M_{A}^{Q E L}$.

The $Q^{2}$ distribution for the QEL sample events was fitted using a dipole description of the axial-vector form factor and making the same assumptions about CVC, charge symmetry and time-reversal invariance that were presented earlier when discussing the QEL scattering cross section. Fermi motion and Pauli blocking effects were taken into account using a correction factor that evolved with $Q^{2}$, although these nuclear effects were not strong given the light deuterium target nuclei.

The authors used a variety of different likelihood functions in the fits; rate-only, shape-only, rate and shape and finally a flux independant likelihood function. The shape-only and flux independant likelihood statistics are defined below in equations 71 and 72 respectively:

$$
\begin{gathered}
\mathscr{L}_{\text {shape }}=\sum_{i=1}^{N_{\text {data }}} W\left(Q_{i}^{2}\right) \ln \left[\frac{\frac{d \sigma}{d Q^{2}}\left(Q_{i}^{2}, E_{\mathrm{v} i} ; M_{A}^{Q E L}\right) R\left(Q_{i}^{2}\right) \Phi\left(E_{\mathrm{v} i}\right)}{\iint \frac{d \sigma}{d Q^{2}}\left(Q_{i}^{2}, E_{\mathrm{v} i} ; M_{A}^{Q E L}\right) R\left(Q_{i}^{2}\right) \Phi\left(E_{\mathrm{v} i}\right) d Q^{2} d E_{\mathrm{v}}}\right] \\
\mathscr{L}_{F I}=\sum_{i=1}^{N_{\text {data }}} W\left(Q_{i}^{2}\right) \ln \left[\frac{\frac{d \sigma}{d Q^{2}}\left(Q_{i}^{2}, E_{\mathrm{v} i} ; M_{A}^{Q E L}\right) R\left(Q_{i}^{2}\right)}{\iint \frac{d \sigma}{d Q^{2}}\left(Q_{i}^{2}, E_{\mathrm{v} i} ; M_{A}^{Q E L}\right) R\left(Q_{i}^{2}\right) d Q^{2}}\right]
\end{gathered}
$$

where $W\left(Q_{i}^{2}\right)$ is the weight due to scanning efficiency, $R\left(Q_{i}^{2}\right)$ is the correction factor accounting for nuclear effects, $\Phi\left(E_{\mathrm{v} i}\right)$ is the neutrino flux and $d \sigma / d Q^{2}$ is the differential cross section. The

\footnotetext{
${ }^{6}$ The kinematics of QEL events are constrained and so the measured information from the main muon track and the incident neutrino can be used to predict the energy and momentum of the outgoing proton. The energy and momentum of the second prong in a candidate QEL event were then be compared to this kinematic prediction for the proton and any differences used to remove background events from the sample.
} 


\begin{tabular}{|c|c|}
\hline Likelihood Type & Best Fit $M_{A}^{Q E L}(\mathrm{GeV})$ \\
\hline \hline Rate & $0.74 \pm 0.12$ \\
\hline Shape & $1.05 \pm 0.05$ \\
\hline Rate and Shape & $1.03 \pm 0.05$ \\
\hline Flux Independant & $1.00 \pm 0.05$ \\
\hline
\end{tabular}

Table 3: Maximum likelihood values for $M_{A}^{Q E L}$, with a dipole axial-vector form factor, as presented in [34].

results from [34] are summarized in table 3 although there are also results presented in two preceeding publications from the Argonne 12-foot bubble chamber; [35] and [36].

Figure 14 shows the $Q^{2}$ distribution of the QEL event sample from the Argonne 12-foot deuterium bubble chamber along with the prediction (based on equation 58) using their best fit $M_{A}^{Q E L}$ value from the flux independant likelihood analysis. It should be noted that the fit was only performed for $Q^{2}>0.05 \mathrm{GeV}^{2}$.

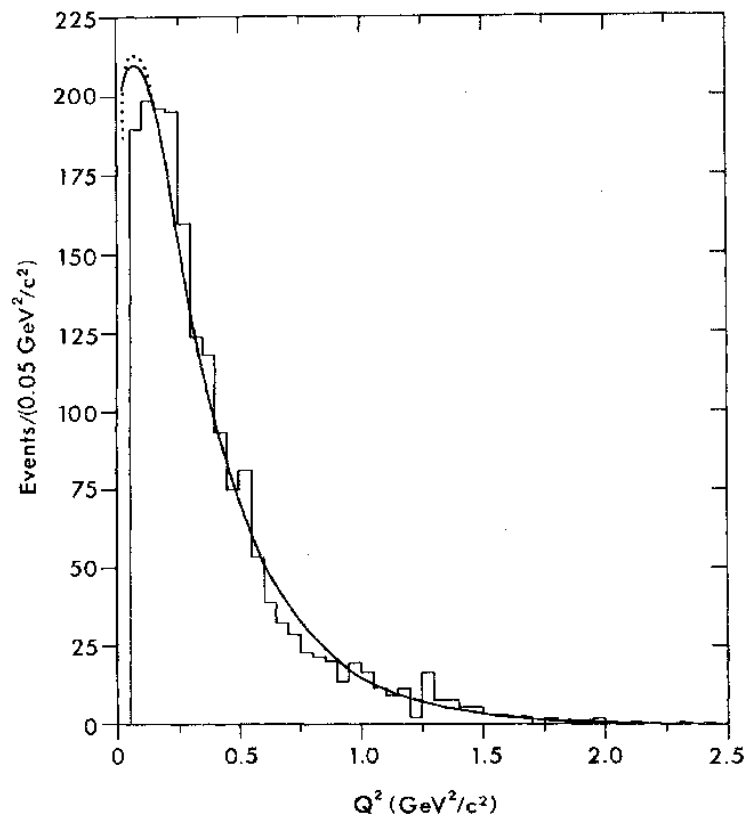

Figure 14: $Q^{2}$ distributions from [34]. The histogram shows the data whilst the solid curve corresponds to a dipole axial-vector form factor with $M_{A}^{Q E L}=1.00 \mathrm{GeV}$. The dotted curve shows an alternate form for the axial-vector form factor with $M_{A}^{Q E L}=1.11 \mathrm{GeV}$. 


\subsection{Results from the K2K Scintillating Fiber Detector}

In 2006 a measurement of $M_{A}^{Q E L}$ for the QEL scattering of muon neutrinos off of oxygen using the K2K Scintillating Fiber ( $\mathrm{SciFi}$ ) detector was presented [37]. The KEK accelerator in Tsukuba, Japan, was used to generate a neutrino beam that passed through a number of 'Near' detectors and the Super-Kamiokande water Čerenkov 'Far' detector. K2K was a long baseline neutrino oscillation experiment but the proximity of the Near detectors to the production point of the neutrino beam allowed for high statistics data samples with which to make neutrino interaction cross section measurements.

The KEK accelerator complex produced a beam of $12 \mathrm{GeV}$ protons that were impinged on an aluminium target. Similar to many experiments that produce neutrino beams, the resulting charged pions and kaons were focussed using two magnetic horns and then allowed to decay to hadrons, leptons and neutrinos. The positively charged hadrons were focussed such that the resulting decay particles travelled towards the Near detectors with a beam dump absorbing all but the muon neutrinos. The neutrino energy spectrum ranged from $0.3 \mathrm{GeV}$ to $5 \mathrm{GeV}$ and peaked at $1.2 \mathrm{GeV}$. The uncertainties on the shape of this spectrum were estimated to be $\pm 20 \%$.

There were a number of different detectors placed 300m downstream of the aluminium target, one of which was the SciFi detector. The SciFi detector consisted of 20 scintillating fiber tracking layers interspersed with water filled aluminium tanks and was read out using CCD cameras. The fiducial mass fraction breakdown was $\sim 70 \% \mathrm{H}_{2} \mathrm{O}, \sim 22 \% \mathrm{Al}$ and $\sim 8 \% \mathrm{CH}$. Unlike the deuterium filled bubble chamber experiment the SciFi detector did not 'see' the spectator nucleons (those nucleons not directly participating in the interaction) in QEL interactions but rather observed the outgoing proton and muon. In practice the analysis event selection introduced a threshold momentum, of $600 \mathrm{MeV}$ for protons and $200 \mathrm{MeV}$ for pions, for the second track to be observable in the detector. As such the K2K QEL scattering analysis used samples of oneand two-track events.

The $M_{A}^{Q E L}$ extraction used the $Q^{2}$ distribution of three distinct samples; the one-track sample and two sub-divisions of the two-track sample. For the two-track sample the reconstructed kinematics of the muon and neutrino were used to predict the kinematics of the outgoing proton 
and then this prediction could be compared to the observed kinematics of the second track. An event selection criterion was placed on how well each two-track event matched the QEL hypothesis allowing for a QEL-enriched two-track sample and a non-QEL two-track sample.

The shapes of the $Q^{2}$ distributions for each of these three samples were fit in separate regions of neutrino energy so as to minimize the systematic error coming from the uncertainties on the incident neutrino flux. The fits used a dipole approximation for the axial-vector form factor (with the usual assumptions about $\mathrm{CVC}$ and the removal of $F_{S}, F_{T}$ and $F_{P}$ ) with $M_{A}^{Q E L}$ as a free parameter and also included a number of systematic parameters. The region below $Q^{2}=$ $0.2 \mathrm{GeV}^{2}$ was excluded in the fit because in this regime the mis-modelling of nuclear effects, which are much more important for QEL scattering off of oxygen as compared to deuterium, constitutes a large uncertainty on the measurement.

The best fit value for the axial-vector mass was $1.27 \pm 0.12 \mathrm{GeV}$ with the dipole approximation for $F_{A}$. The main result quoted in [37] used the updated BBA-2003 Form Factors which gave a best fit $M_{A}^{Q E L}$ of $1.20 \pm 0.12 \mathrm{GeV}$ and this result which was shown to be robust under reasonable changes to the analysis selection criteria and fitted range in $Q^{2}$. Although many more events were used in this analysis, compared to the Argonne results, the quoted error is larger. This is probably due to the extremely high purity of the QEL event sample collected in the bubble chamber and it's enhanced resolution for kinematic variables such as $Q^{2}$. Figure 15 shows the $Q^{2}$ distributions of the QEL event samples for the first part of the K2K data taking, 'K2K-I', along with curves generated with a Monte Carlo simulation of the experiment that used the best fit $M_{A}^{Q E L}$ value.

The one-track and two-track QEL enhanced samples have the highest purity but figure 15 shows that there are relatively large contributions from backgrounds. The dominant background comes from resonant single pion production, such as $\nu_{\mu} p\left(\rightarrow \Delta^{++}\right) \rightarrow \mu^{-} p \pi^{+}$. In such events a final state particle can either be below threshold for detection or be re-absorbed in the nucleus and both these processes will lead to a two-prong event being observed in the detector. The kinematic matching to the QEL hypothesis can remove some events from the two-track QEL enhanced sample although intranuclear re-scattering of the final state particles can change 

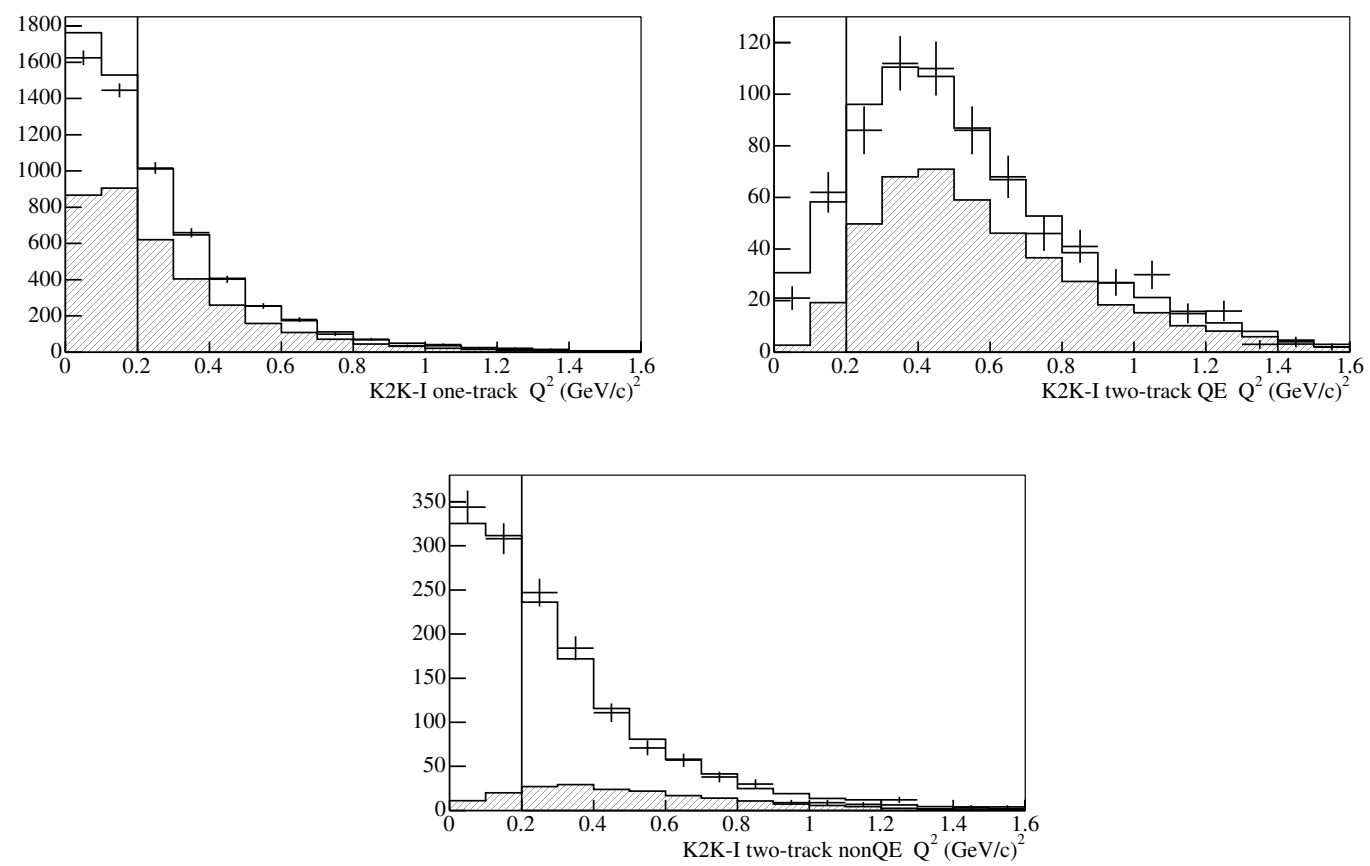

Figure 15: $Q^{2}$ distributions from [37]. The points with errors show the data for each of the three subsamples whilst the larger solid histograms show the prediction from a Monte Carlo simulation generated using a dipole axial-vector form factor with $M_{A}^{Q E L}=1.20 \mathrm{GeV}$ and BBA-2003 vector form factors. The smaller solid histograms show the true QEL events in each of the simulated sub-samples.

the observed kinematics. In fact these single pion events are kinematically very similar to QEL scattering events and have their own axial-vector form factor and associated axial vector mass, $M_{A}^{1 \pi}$. This suggests a possible explanation for the difference in $M_{A}^{Q E L}$ values reported by the deutrium experiments and $\mathrm{K} 2 \mathrm{~K}$, namely that the lower purity $\mathrm{K} 2 \mathrm{~K}$ samples are being fitted for $M_{A}^{Q E L}$ but are 'seeing' some of the value of $M_{A}^{1 \pi}$ in this measurement.

\subsection{Results from the MiniBooNE Experiment}

One of the most recent measurements of $M_{A}^{Q E L}$ came in June 2007 from the MiniBooNE collaboration [38]. The MiniBooNE experiment at FNAL uses a beam of muon neutrinos incident on a detector filled with 800 tons of mineral oil, $\mathrm{CH}_{2}$. The FNAL booster accelerator provides 8.89 $\mathrm{GeV}$ protons which are brought into collision with a beryllium target located inside a magnetic horn. The magnetic field focusses the $\pi^{+}$and $K^{+}$such that when they decay the trajectory of the muon neutrinos passes through the detector. The MiniBooNE beam provides a mean 
neutrino energy of $0.7 \mathrm{GeV}$ with $99 \%$ of the neutrino energies below $2.5 \mathrm{GeV}$. The detector is a spherical tank filled with $\mathrm{CH}_{2}$ and instrumented with photomultiplier tubes (PMTs) to collect the Čerenkov light produced by the particles produced in the neutrino-carbon interactions.

MiniBooNE identifies QEL events by measuring the primary muon from the scattering and then the electron from the subsequent decay of this muon; $\mu^{-} \rightarrow e^{-} v_{\mu} \bar{v}_{e}$. This technique helps to remove single $\pi^{+}$resonant events where a second electron is detected from the decay chain of the pion. The MiniBooNE event sample consisted of 193709 events and was estimated to have an QEL efficiency of 35\% and a QEL purity of 74\%.

The MiniBooNE neutrino interaction simulation uses a Relativistic Fermi Gas (RFG) model for the scattering of neutrinos from carbon nuclei. The $M_{A}^{Q E L}$ extraction proceeds in a similar way to the previous analyses in that the $Q^{2}$ distribution of the QEL events is fitted using $M_{A}^{Q E L}$ as a free parameter and the Dipole Form Factors are used. This analysis is slightly different though and includes a second free parameter, $\kappa$, which is used to control the amount of Pauli blocking at low values of $Q^{2}$. MiniBooNE fits the shape of the $Q^{2}$ distribution all the way down to $Q^{2}=0 \mathrm{GeV}^{2}$ and obtains best fit values of $M_{A}^{Q E L}=1.23 \pm 0.20 \mathrm{GeV}$ and $\kappa=1.019 \pm 0.011$. Figure 16 is taken from [38] and shows the data and best fit MC $Q^{2}$ distributions along with a $1 \sigma$ confidence level contour as a function of $M_{A}^{Q E L}$ and $\kappa$.

The best fit $M_{A}^{Q E L}$ accomdates the $Q^{2}$ behaviour of the QEL event sample at higher values of $Q^{2}$ whereas the Pauli blocking suppression factor $\kappa$ allows the fit to include the low $Q^{2}$ region (below $0.2 \mathrm{GeV}^{2}$ ). In this measurement $M_{A}^{Q E L}$ should be considered to be an effective parameter because it is likely accounting for some mis-modelled feature in the RFG simulation such as the momentum distribution of nucleons within the carbon nuclei. The following section will comment further on the interpretation of $M_{A}^{Q E L}$ measurements and comparisons between the various experiments.

\subsection{Summary of Current Knowledge and World-Average Values}

Table 4 summarises many of the $M_{A}^{Q E L}$ measurements that have been made previously along with the target nuclei for each experiment. The weighted average for $M_{A}^{Q E L}$ from neutrino 


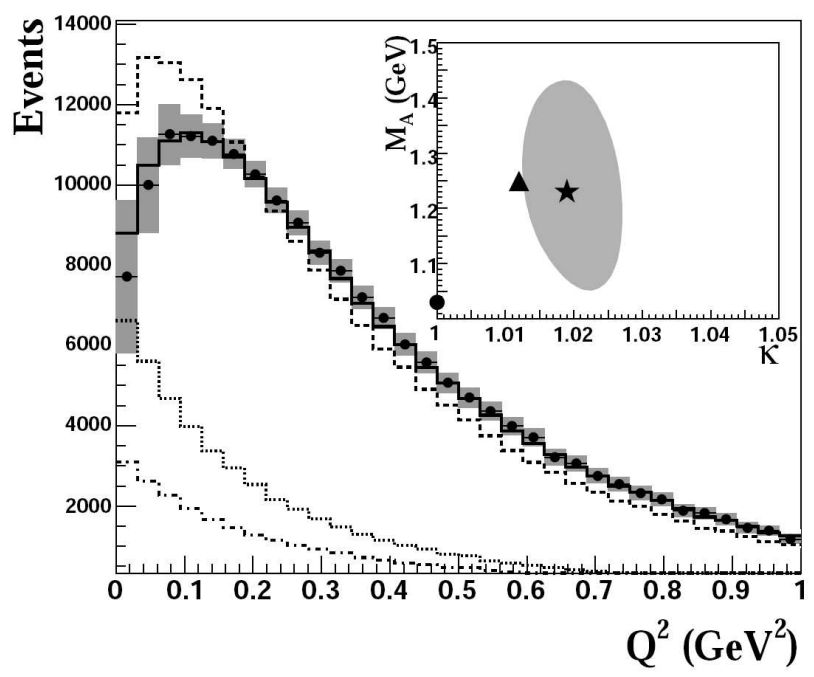

Figure 16: $Q^{2}$ distributions from [38]. The dashed histogram shows the output of a Monte Carlo simulation of the experiment before the fit whilst the solid histogram shows the simulated prediction with the best fit parameter values applied. The dotted and dot-dash histograms show the backgrounds that are not CC QEL and not CC QEL-like respectively and the points show the data. The inset figure shows the $1 \sigma$ confidence contour as a function of $M_{A}^{Q E L}$ and $\kappa$ with the star showing the best fit point, the circle showing the starting values and the triangle showing the fit results after varying the background shape in $Q^{2}$.

scattering experiments, as compiled in 2001 [46] (and hence not including the K2K and MiniBooNE measurements), and using the data selection criteria of the Particle Data Group (PDG) [47], was $1.026 \pm 0.021 \mathrm{GeV}$.

There are a number of factors that need to be taken into consideration when comparing measurements of $M_{A}^{Q E L}$ including the nuclear target, analysis methodology, flux and background cross section uncertainties and the set of form factors used. Depending on these particulars, $M_{A}^{Q E L}$ measurements should often be considered as effective measurements where the extracted value is also accounting for some other physics effects. The above sections introduced some of these factors and their importance is summarised below:

- Target Nucleus: For heavier target nuclei the modelling of nuclear effects has large uncertainties at low $Q^{2}$. Most previous fits for $M_{A}^{Q E L}$ have used a lower cut-off in $Q^{2}$ and a large number of these measurements were shown to be consistent under small changes 


\begin{tabular}{|c|c|c|c|}
\hline Experiment & Target & $M_{A}^{Q E L}(\mathrm{GeV})$ & Comments \\
\hline \hline ANL 1982[34] & $\mathrm{D}$ & $1.00 \pm 0.05$ & Dipole \\
\hline FNAL 1983[39] & $\mathrm{D}$ & $1.05_{-0.16}^{+0.12}$ & Dipole \\
\hline BNL 1990[40] & $\mathrm{D}$ & $1.07_{-0.045}^{+0.040}$ & $\begin{array}{c}\text { Dipole } \\
M_{A}^{1 \pi}=1.28_{-0.10}^{+0.08} \mathrm{GeV}\end{array}$ \\
\hline CERN 1977[41] & $\mathrm{CF}_{3} \mathrm{Br}$ & $0.94 \pm 0.17$ & Dipole \\
\hline SKAT 1990[42] & $\mathrm{CF}_{3} \mathrm{Br}$ & $1.05 \pm 0.14$ & Dipole \\
\hline SKAT 1990[42] & $\mathrm{CF}_{3} \mathrm{Br}$ & $0.79 \pm 0.20$ & Dipole, anti-neutrino \\
\hline BNL 1969[43] & $\mathrm{Fe}$ & $1.05 \pm 0.20$ & Dipole \\
\hline BNL 1987[44] & $\mathrm{HC}, \mathrm{Al}^{2}$ & $1.06 \pm 0.05$ & Dipole, NC elastic scattering \\
\hline BNL 1988[45] & $\mathrm{HC}_{1} \mathrm{Al}$ & $1.09 \pm 0.04$ & Dipole, anti-neutrino \\
\hline K2K 2006[37] & $\mathrm{H}_{2} \mathrm{O}, \mathrm{Al}$ & $1.20 \pm 0.12$ & BBA-2003 \\
\hline K2K 2006[37] & $\mathrm{H}_{2} 0, \mathrm{Al}$ & $1.27 \pm 0.12$ & Dipole \\
\hline MiniBooNE 2007[38] & $\mathrm{CH}$ & $1.23 \pm 0.20$ & Dipole, $\kappa=1.019 \pm 0.011$ \\
\hline
\end{tabular}

Table 4: $M_{A}^{Q E L}$ measurements from previous measurements grouped by target nucleus. The values given all come from pure shape information (shape-only or flux independant) fits to the $Q^{2}$ distributions although some references also quota results from shape and rate fits. The comments column includes the type of weak vector form factors used for each measurement.

to the cut-off value. It is not yet completely clear whether $M_{A}^{Q E L}$ should be a constant for all nuclei or take different values depending on the nucleus in question. Furthermore the form of the axial-vector form factor itself could potentially be slightly different for different target nuclei. It should be noted that a significant benefit of using heavier target nuclei is a much increased rate of neutrino interactions.

- Fit Methodology: Previous measurements have used one or more of several different methods to extract $M_{A}^{Q E L}$ from their QEL scattering data; rate-only, shape-only, rate and shape, flux independant. The rate and shape method uses the most information although can be susceptible to uncertainties in background levels and the incident neutrino flux. Most previous measurements that use multiple methods find consistent values for $M_{A}^{Q E L}$ (within errors) but there are some exceptions.

- Flux Uncertainties: Many previous experiments did not have sufficient knowledge of the incident neutrino flux to perform a fit that included rate information. The shape- 
only and flux independant methods minimise the effect of such uncertainties (as the $Q^{2}$ distributions for QEL scattering events are not very dependant upon $E_{v}$ ) although the dependance increases at low values of $Q^{2}$.

- Background Cross Section Uncertainties: The deuterium bubble chamber experiments achieved very high QEL purities and so mostly eliminated the effect of uncertainties on the levels of background in their samples. However, for the other experiments the uncertainties in the cross sections (and cross section shapes) for the background processes contributed significantly to the errors on the best fit $M_{A}^{Q E L}$ values. The dominant background for QEL scattering is resonant single pion production events which have a corresponding $M_{A}^{1 \pi}$. Experiments with lower purity could have an artifically high or low extracted $M_{A}^{Q E L}$ value due to the presence of such events.

- Vector Form Factors: Most previous measurements of $M_{A}^{Q E L}$ have used the dipole approximation for the weak vector form factors of the nucleon. However more recent work, such as presented in [32], has suggested alternate forms for these form factors. The extraction of $M_{A}^{Q E L}$ is sensitive to both the set of vector form factors used and also to the constants, such as $F_{A}\left(Q^{2}=0\right)$, whose world average values have changed over time.

- Standard Assumptions: Assumptions, such as those of CVC and PCAC, are often made and imply that $M_{A}^{Q E L}$ is the only free parameter in the differential cross section for QEL scattering with respect to $Q^{2}$. Another assumption that is often made is a dipole description of the axial-vector form factor. Some previous experiments have also tried fitting alternate expressions for $F_{A}\left(Q^{2}\right)$ and such choices can effect the extracted $M_{A}^{Q E L}$ value. 


\section{The MINOS Experiment}

MINOS [48] is a long baseline neutrino oscillation experiment that uses a neutrino beam produced by the NuMI [49] facility at FNAL. The neutrinos pass through a Near Detector (ND) about $1 \mathrm{~km}$ downstream of the production target and then through the Far Detector (FD) a further $734 \mathrm{~km}$ away in the Soudan Mine in northern Minnesota. The general layout of the MINOS experiment is shown in figure 17. This chapter will discuss in detail the NuMI beamline, the MINOS detectors and the physics goals for the experiment.

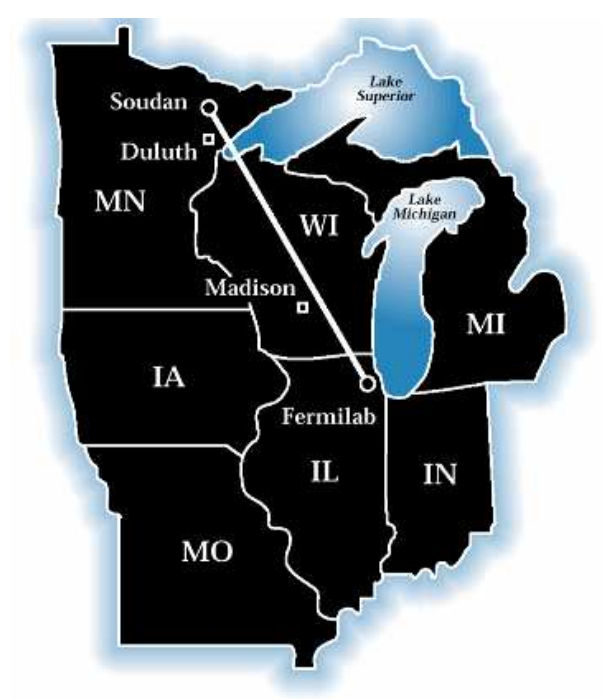

Figure 17: Overview of the MINOS experimental layout. Image courtesy of [50].

\subsection{The NuMI Beamline}

The first stage in the production of the NuMI neutrino beam is the acceleration of protons using a linear accelerator (Linac), the Booster circular accelerator and finally the Main Injector (MI) circular accelerator. The Linac takes the protons to a momentum of $400 \mathrm{MeV} / \mathrm{c}$, the Booster brings this up to $8 \mathrm{GeV} / \mathrm{c}$ and finally the MI forms the protons into batches and accelerates them to a momentum of $120 \mathrm{GeV} / \mathrm{c}$. For the majority of the first year of NuMI running the MI contained seven batches, five of which were earmarked for the NuMI primary-proton line and two of which were destined for the Tevatron accelerator. 
The whole acceleration process takes $1.6 \mathrm{~s}$ after which the protons are extracted from the MI using 'kicker' magnets and this beam is bent downward at an angle of $58 \mathrm{mrad}$, in order to point towards the Soudan mine, and sent $350 \mathrm{~m}$ to the NuMI target. The proton batches were extracted in an $8.6 \mu$ s spill with a typical spill containing about $2.1 \times 10^{13}$ protons and a typical repetition rate for the machine of about 2.2s. The NuMI beam has been designed to provide an average beam power of up to $400 \mathrm{~kW}$. Figure 18 shows an overhead view of the FNAL accelerator complex.

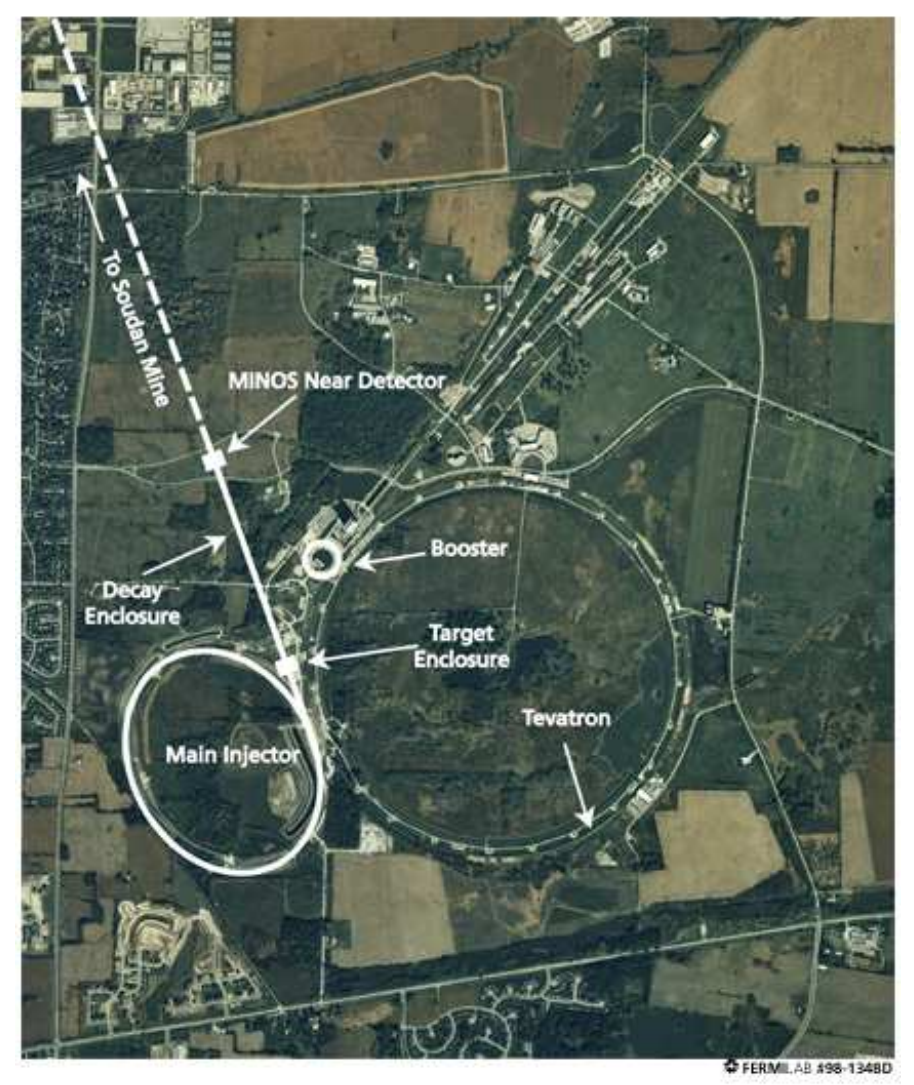

Figure 18: Overhead view of the FNAL accelerator complex. Image courtesy of [50].

The NuMI target is a water-cooled graphite rod (shown in figure 19). The target dimensions are $6.4 \times 15 \times 940 \mathrm{~mm}^{3}$ and the target is segmented longitudinally into forty-seven fins. The target is designed to be narrow so as to minimize the re-absorption of particles produced in the primary proton collision whilst being long enough to maximise hadron production. 


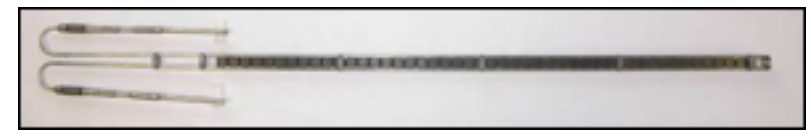

Figure 19: Photograph of the NuMI graphite target. Photograph courtesy of [50].

The proton collisions with the carbon produce a spray of particles, mainly pions and kaons. These secondary particles are focused (or de-focused depending on the experimental setup and the particle charge) by a pair of parabolic focussing 'horns'. The horns are pulsed with a nominal current of $200 \mathrm{kA}$ to produce a toroidal magnetic field with a maximum strength of $30 \mathrm{kG}$. The horns act as magnetic lenses that sign-select and momentum-select the secondary hadrons. Figure 20 shows photographs of the NuMI horns.


Figure 20: Photographs of the NuMI parabolic focussing horn inner conductors (left) and bottom-view (right). Photographs courtesy of [50].

After being focussed by the horns the charged hadrons enter a $675 \mathrm{~m}$ long, $2 \mathrm{~m}$ diameter evacuated decay pipe in which many of them decay to neutrinos through processes such as $\pi^{+}\left(K^{+}\right) \rightarrow$ $\mu^{+} v_{\mu}$. Hadrons that make it through this decay volume are stopped by a $5 \mathrm{~m}$ hadron absorber consisting of water-cooled aluminium core surrounded by steel blocks and then a layer of concrete.

Finally the remaining muons interact in the $300 \mathrm{~m}$ of dolomite rock that lies between the hadron 
absorber and the ND cavern leaving a beam of neutrinos whose path takes them through the MINOS detectors. Figure 21 shows the various components of the NuMI beamline. The neutrino beam comprises of $97.8 \% v_{\mu}$ with the major contaminations of $1.8 \% \bar{v}_{\mu}$ coming from $\mu^{+}$decays and target-produced $\pi^{-}$decaying to $\mu^{-} \bar{v}_{\mu}$ and $0.4 \% v_{e}$ coming from $\mu^{+}$decays and target-produced $K_{e 3}^{+}$decays (these numbers are based on a Monte Carlo simulation of the neutrino beamline and the hadron production off of the NuMI target).

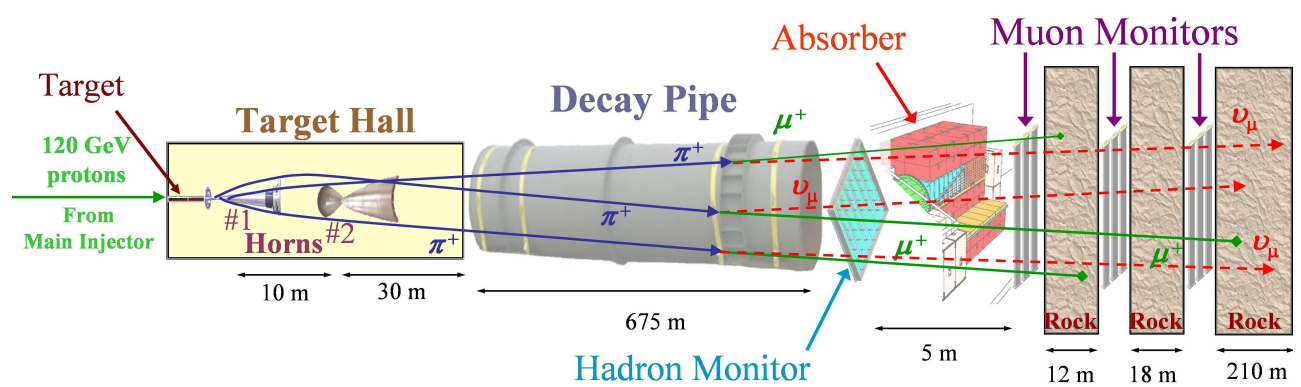

Figure 21: The components of the NuMI beamline. Image courtesy of [51].

The NuMI beam has a lot of flexibilty and can be setup to change the neutrino energy spectrum seen by the MINOS detectors. Different neutrino energy spectra can be produced by moving the NuMI target with respect to the first focussing horn or changing the magnetic field produced by the horn by varying the electric current. Both methods result in different momentum hadrons being focussed which leads to different energies of the resulting neutrinos. Table 5 lists the different beam configurations in which MINOS has taken data. In addition to changing the neutrino energy spectra the NuMI horn current could be reversed in the future to produce a beam comprised predominantly of $\overline{\mathrm{v}}_{\mu}$.

Figure 22 shows the weekly and total integrated protons-on-target (POT) delivered by the NuMI beamline (in all beam configurations) from May 2005 through to March 2006. The dataset used for the analysis presented in this thesis is taken from $20^{\text {th }}$ May 2005 through to $25^{\text {th }}$ February 2006 and corresponds to an exposure of $\sim 1.27 \times 10^{20}$ POT in the L010z185i beam configuration. 


\begin{tabular}{|c|c|c|c|c|}
\hline $\begin{array}{c}\text { Beam } \\
\text { Configuration }\end{array}$ & $\begin{array}{c}\text { Target } \\
\text { Position }(\mathrm{cm})\end{array}$ & $\begin{array}{c}\text { Horn } \\
\text { Current }(\mathrm{kA})\end{array}$ & $\begin{array}{c}\text { Most Probable } \\
E_{\mathrm{v}} \pm \mathrm{RMS} \\
(\mathrm{GeV})\end{array}$ & $\begin{array}{c}\text { Exposure } \\
\left(10^{18} \text { POT }\right)\end{array}$ \\
\hline \hline LE10/0kA & 10 & 0 & $7.4 \pm 4.1$ & 2.69 \\
\hline LE10/170kA & 10 & 170 & $3.1 \pm 1.1$ & 1.34 \\
\hline LE10/185kA & 10 & 185 & $3.3 \pm 1.1$ & 127. \\
\hline LE10/200kA & 10 & 200 & $3.5 \pm 1.1$ & 1.26 \\
\hline LE100/200kA & 100 & 200 & $5.6 \pm 1.5$ & 1.11 \\
\hline LE250/200kA & 250 & 200 & $8.6 \pm 2.7$ & 1.55 \\
\hline
\end{tabular}

Table 5: Beam configuration definitions and exposures, quantified by protons-on-target (POT), for the corresponding datasets as recorded in the first year of running. The majority of NuMI running uses the LE10/185kA configuration as the resulting neutrino flux is maximised in the region of interest suggested by the Super-Kamiokande best fit oscillation parameters. The LE10/OkA beam configuration has the broadest neutrino energy distrubution as the target-produced charged hadrons are not focussed. The mean and RMS neutrino energies are calculated using a Monte Carlo simulation of the NuMI beamline and the charged-current cross section.

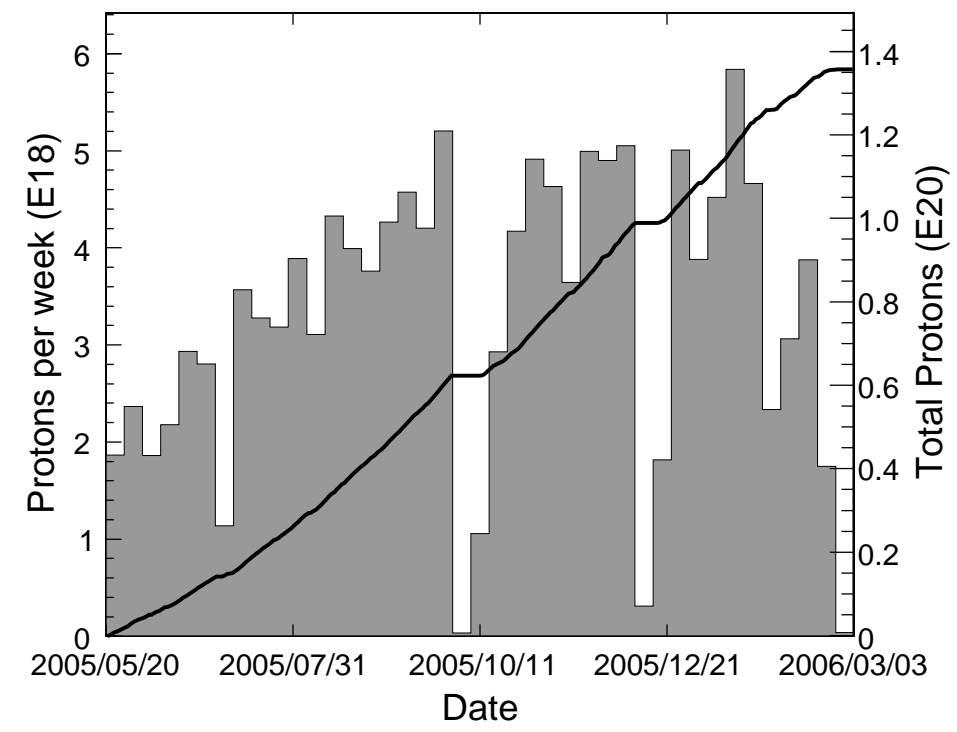

Figure 22: Weekly and integrated POT delivered by NuMI during it's first year of operation. Figure taken from [11]. 


\subsection{The MINOS Detectors}

MINOS uses three detectors; the Near and Far detectors measure the NuMI neutrino interactions whilst the smaller Calibration detector (CalDet) was exposed to a test beam at the CERN Proton Synchrotron (PS). The results from CalDet characterise the response of the MINOS detectors to electrons, muons, protons and pions.

The MINOS detectors are designed to be functionally identical in order to minimise the systematic uncertainties involved in the two-detector oscillation analysis but do have differences stemming from the different environments in which they must operate. This section will describe the common features of the MINOS detectors before focussing on the particulars of the Near, Far and Calibration detectors.

\subsubsection{Common Features of the Detectors}

The MINOS detectors are steel-scintillator tracking calorimeters. Both the Near and Far detectors are magnetised with toroidal fields, with a field strength of up to $1.5 \mathrm{~T}$, to allow momentum and charge-sign measurements of the muons produced in $v_{\mu} N$ interactions.

Each detector consists of a number of steel-scintillator 'planes'. Each plane consists of a $2.54 \mathrm{~cm}$ thick plate of steel attached to a $1.0 \mathrm{~cm}$ thick layer of scintillator. The detectors consist of large numbers of planes which are hung perpendicular to the incident neutrinos with a $2.4 \mathrm{~cm}$ air gap between each plane. The higher density steel provides the main medium in which the neutrinos will interact whilst the scintillator constitutes the active element of the detectors.

The scintillator layers consist of $1.0 \mathrm{~cm} \times 4.1 \mathrm{~cm}$ cross-section strips of polystyrene that can measure up to $8 \mathrm{~m}$ in length and are doped with the fluors PPO (1\%) and POPOP $(0.03 \%)$. The strips are arranged side-by-side and then encased in aluminium to form light-tight scintillator modules which can be mounted on the steel sheets. Strips in adjacent planes are oriented orthogonal to one another to allow for a three-dimensional reconstruction of neutrino interactions in the detectors.

Particles traversing the scintillator strips produce scintillation light which is collected by $1.2 \mathrm{~mm}$ 
diameter wavelength-shifting (WLS) optical fibres. Each strip has a $2 \mathrm{~mm}$ deep groove running along one side into which the WLS fibres are glued. The WLS fibres shift the average wavelength of photons from blue $(\lambda=460 \mathrm{~nm})$, which are emitted from the scintillator, to green $(\lambda=530 \mathrm{~nm})$.

The strips have a $\mathrm{TiO}_{2}$ doped outer reflective coating which helps to trap and reflect scintillation light until it is collected by the WLS fibres and routed out of the planes to be measured. After exiting the strips the WLS fibres are collected in a manifold before terminating in an optical connector. Figure 23 shows an individual scintillator strip along with a collection of strips arranged into a module.



Figure 23: Photograph of a single scintillator strip containing a WLS fibre along with a photograph of a collection of strips mounted side-by-side in a module. The WLS fibres are collected by the manifold at the bottom of the picture to be routed out to the optical connector. Photographs courtesy of [50].

After reaching the optical connector photons are routed via clear optical fibres, which have a much longer attenuation length, to multi-anode photomultiplier tubes (PMTs). The PMTs convert the collected photons to an electrical signal which can then be processed by the data acquisition (DAQ) systems. Figure 24 shows a schematic of a scintillator plane (in the FD) and it's readout.

The two main MINOS detectors each have specially designed front-end electronics which will be described in the following sections. The detectors need different electronics to handle their vastly different event rates; the ND is situated close to the neutrino production point and, in the 


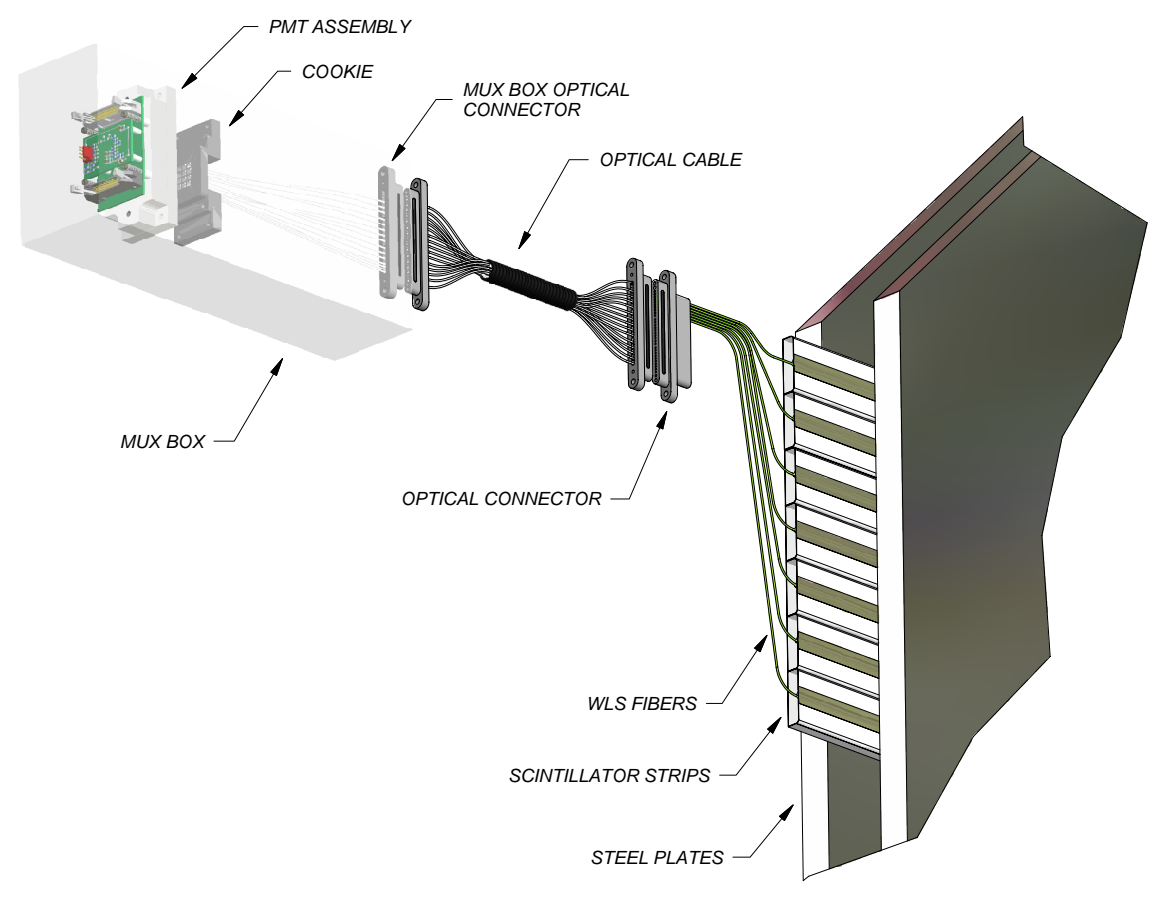

Figure 24: Optical readout of a MINOS detector scintillator plane. The scintillation light from particle interactions is collected by WLS fibres and then, through optical connectors and clear optical fibres, routed to PMTs. The multiplex (MUX) box will be discussed further in the section describing the FD. This image was taken from [11].

L010z185i beam configuration, observes activity from an average of 16 neutrino interactions per spill whereas at the FD the beam has diverged to a width of $\sim 1 \mathrm{~km}$ with the FD observing only a few neutrino interactions per day.

\subsubsection{The Far Detector}

The $5.4 \mathrm{kT}$ Far detector is the largest of the three MINOS detectors and is situated $705 \mathrm{~m}$ underground at the Soudan Underground Laboratory in Minnesota, USA. The detector consists of a total of 484 octagonal planes measuring $8 \mathrm{~m}$ across. The detector is divided into two 'supermodules', of 249 and 237 planes, seperated by an air gap of $1.2 \mathrm{~m}$ because of restrictions on the length of the magnetic coil. As such each super-module is independantly magnetised by a $15 \mathrm{kA}$ field-coil running though the centre of the detector which provides an average field of 1.27 $\mathrm{T}$ in the steel. Figure 25 shows a photograph of the completed FD. 


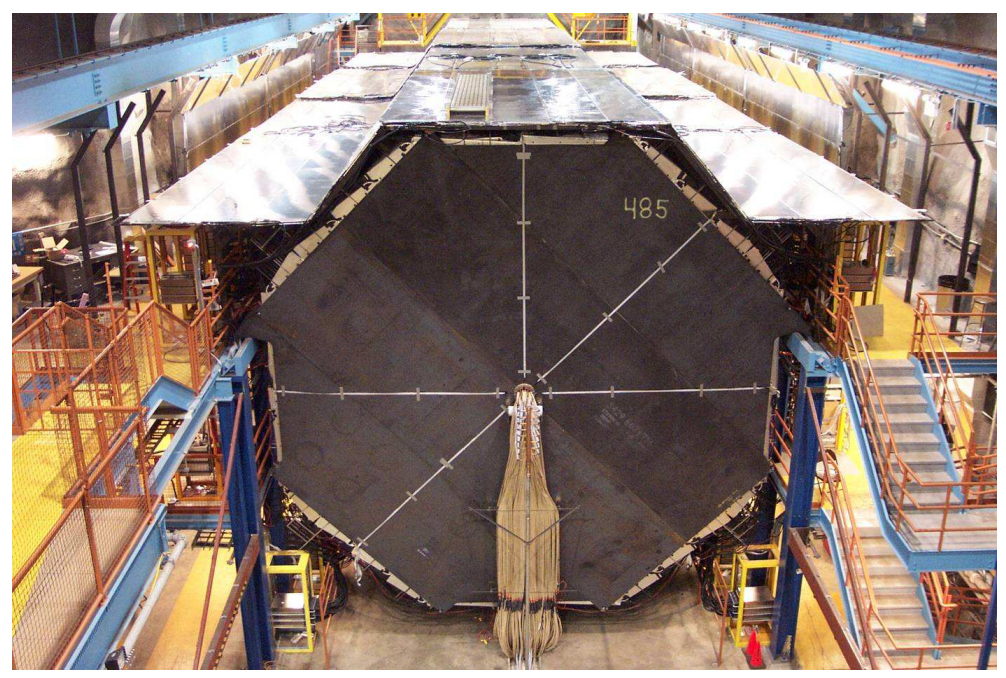

Figure 25: Photograph of the Far detector. The magnetic coils can be seen entering the centre of the first $8 \mathrm{~m}$ diameter octagonal plane. The PMTs and readout electronics are situated at the left-and right-hand sides of the detector along the walkways. The cosmic ray veto shield can be seen above the detector. Photograph courtesy of [50].

Each plane has a total of 192 scintillator strips arranged side-by-side and oriented at $45^{\circ}$ to the vertical which are readout at both ends by Hamamatsu M16 PMTs. There are a total of 185856 strip-ends in the FD and to cope with this large number of channels the fibres from eight different strips are readout by each PMT pixel using a 'multiplexing' technique. In order to resolve the ambiguity in which strips were actually hit the strip-to-pixel pattern is different for each side of the detector. This complex optical 'summing' is performed inside multiplexing (MUX) boxes with each MUX box containing three PMTs and reading-out one side of two planes.

The front-end electronics at the FD is based on a multi-channel application-specific integrated circuit (ASIC) known as a 'VA chip' with one VA chip servicing each PMT. The electronics at the FD is continuously live and each PMT can trigger independantly. The VA chips digitise the signals from the PMTs with a precision of $2 \mathrm{fC}$ and are triggered when the summed signal from all the pixels of a single PMT (the dynode signal) exceeds a threshold of $\sim 0.25$ photoelectrons. In addition the front-end electronics time-stamp the signal with a least significant bit of 1.56 ns. 
These signals are pedestal suppresed and passed to a PC farm which then apply some higherlevel triggering conditions to decide whether the data is saved. During data taking the main triggering condition is known as the 'spill trigger' which causes the FD to be readout coincident with a beam spill from the MI. The Near and Far detectors are synchronised using Global Positioning System (GPS) clocks and when a beam spill occurs at FNAL a time-stamp is sent via the internet to the FD which then records all hits in a $\pm 40 \mu$ s window centred about the spill time. This 'spill-window' is extended to ensure that it is bounded by an activity-free period of at least $156 \mathrm{~ns}$ so as not to split candidate neutrino events.

The FD is equipped with a veto shield positioned above the detector (see figure 25). The veto shield provides a vital rejection of cosmic ray events which form a background to the analysis of atmospheric neutrino interactions. MINOS has already published results [52] on the oscillation parameters measured using atmospheric neutrino interactions in the FD.

\subsubsection{The Near Detector}

The Near detector has a mass of $0.98 \mathrm{kT}$ and is located in a specially contructed cavern 98 $m$ underground on-site at FNAL. The ND was designed to cope with a large rate of neutrino interactions (the neutrino beam has a divergence of only $\sim 0.5 \mathrm{~m}$ at the detector). The ND consists of 282 planes arranged into a single super-module and has an asymmetric octagonal shape with each plane being $6.2 \mathrm{~m}$ wide and $3.8 \mathrm{~m}$ high. There is a $30 \times 30 \mathrm{~cm}^{2}$ hole offset $0.56 \mathrm{~m}$ from the horizontal centre of the detector through which the magnetic field-coil passes. The neutrino beam spot lies $0.93 \mathrm{~m}$ from the horizontal centres of the planes (in the direction opposite to the coil) where the 40kA field-coil provides a magnetic field of $1.17 \mathrm{~T}$. Figure 26 shows a photograph of the ND taken during it's installation.

The ND is seperated into two parts; the upstream 120 planes constitute the calorimeter section in which every steel plane is instrumented with scintillator whilst the remaining downstream planes constitute the spectrometer section where only one in five planes is instrumented. The calorimeter section is used to precisely measure the interaction vertices and toplogies whereas the spectrometer section is used to measure the momentum of the produced muons via their range (if they stop in the detector) or their curvature in the magnetic field (if they exit the 




Figure 26: Photograph of the Near detector during installation. The coil hole can be seen along with an aluminium-covered scintillator module, the module does not extend the entire length of the steel as this is a partially-instrumented plane. The racks on the right-hand side run along one side of the detector and hold the PMTs and readout electronics. Photograph courtesy of [50].

detector).

One further difference in the two sections is that whilst one in five of all of the 284 planes are fully instrumented the remaining four out of five calorimeter planes are only partially instrumented (see figure 26). This partial instrumentation is adaquate for measuring the development of hadronic showers from the neutrino interactions (as they are centred about the beam spot). Figure 27 shows a schematic of the various sections of the ND.

The scintillator strips in the ND are shorter than those at the FD and the planes are readout at one end (the opposite strip-ends are coated with a reflective material to increase the level of light being readout). ND strips are readout using Hamamatsu M64 multi-anode PMTs with partially instrumented planes requiring one PMT (64 pixels for 64 strips) and fully instrumented planes requiring $1 \frac{1}{2}$ PMTs to readout their 96 strips. In the spectrometer section the amount of intrumentation required is reduced by summing the signal from sets of four adjacent pixels on each PMT into a single electronics channel. The adjacent pixels are not connected to adjacent strips and so the four-fold ambiguity can be resolved by tracking events in the calorimeter and projecting the events into the spectrometer to select one of the four possibilities. As such the 


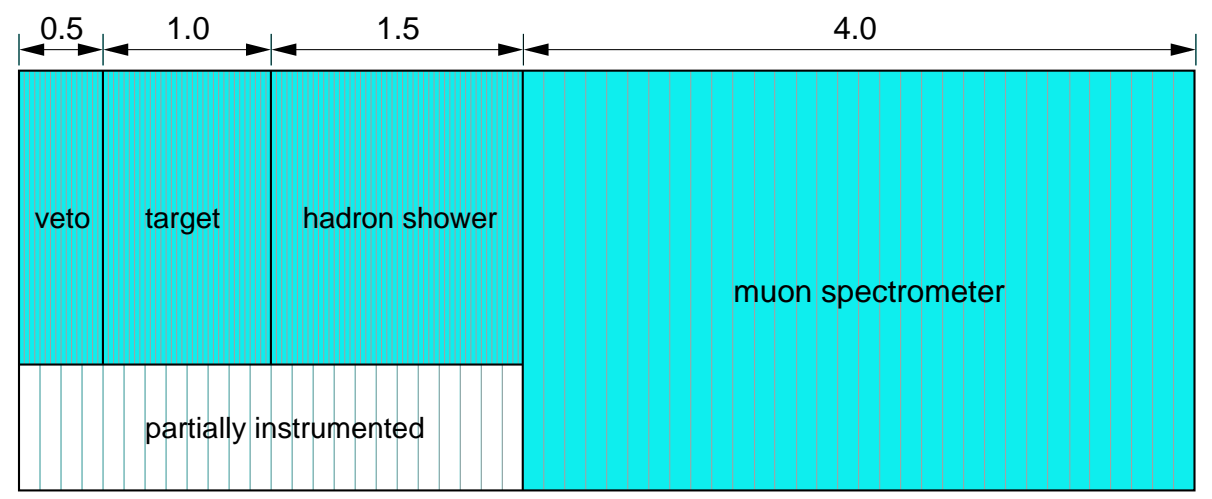

Figure 27: Sections of the Near detector. The calorimeter section can be further divided into the veto section (planes 0-20), the target section (planes 21-60) and the hadron shower section (planes 61-120). Events from the veto section can consist of muons from neutrino interactions in the rock upstream of the detector and can be removed due to the lack of knowledge of the hadronic shower. Events in the target section will be well measured and can be used in comparisons of the neutrino energy spectra at the two detectors. The hadronic shower section contains the showers whilst the spectrometer section is used to provide estimates of the muon energy. Diagram taken from [53].

ambiguity cannot be resolved for events whose vertices are not located inside the calorimeter section.

There are an average of sixteen events occurring in the ND for each beam spill (in the L010z185i beam configuration) and so special high-speed and deadtime-less front-end electronics are used. The system is based on the QIE chip that was developed at FNAL and used by the $\mathrm{CDF}$ and KTEV experiments. In the primary triggering mode, the spill-trigger, the output from every photomultiplier pixel is readout from $1.5 \mu$ s before the beam spill arrival time at the ND continuously for a total of $13 \mu \mathrm{s}$. There is also a dynode trigger mode which is used when taking cosmic ray data in the ND. Further details of the ND electronics can be found in [54].

\subsubsection{The Calibration Detector}

The 12 tonne Calibration detector was constructed to measure the response and topology of the main MINOS detectors to hadronic and electromagnetic interactions. It was operated during 2001-2003 and was exposed to beams of $p^{+}, e^{ \pm}, \mu^{ \pm}$and $\pi^{ \pm}$in the few-GeV regime at the CERN PS accelerator. 
CalDet consisted of sixty $1 \mathrm{~m}$ square planes with each plane having twenty-four scintillator strips. CalDet was operated with electronics and readout that simulated that of both the Near and Far detectors (sometimes simultaneously with each strip-end being readout by either the ND or FD setup). CalDet was also instrumented with a time-of-flight system (to act as a trigger and discriminate between $e, \pi$ and $p$ ) and Čerenkov counters that were used to tag electrons in the detector. CalDet's input to the calibration of the main MINOS detectors will be discussed further in the next chapter. Figure 28 shows a photograph of the calibration detector.

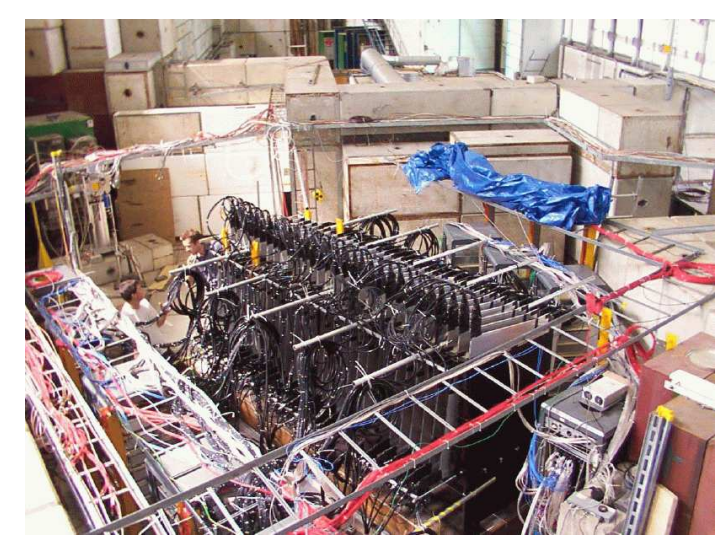

Figure 28: Photograph of the Calibration detector. The readout of the strips was setup to simulate the configurations of both the ND and FD including using optical fibres with lengths closely approximating those used at the detectors. These optical fibres can be seen at the left of the detector. Photograph courtesy of [50].

\subsection{MINOS Physics Goals}

The main physics goal of the MINOS experiment is to establish neutrino oscillations as the mechanism responsible for the apparent loss of atmospheric $v_{\mu}$ and to precisely measure the parameters governing these oscillations; the mass splitting $\Delta m_{32}^{2}$ and the mixing angle $\theta_{23}$. This measurement compares the neutrino energy spectra at the Near and Far detectors in order to search for an energy dependant disappearance of $v_{\mu}$. The latest MINOS results on the atmospheric neutrino oscillation parameters using NuMI neutrino interactions in the MINOS detectors are presented in [11]. 
It is expected that the 'missing' $v_{\mu}$ are predominantly oscillating to $v_{\tau}$. The region of oscillation parameter space indicated by the results from Super-Kamiokande [7] suggests an oscillation maximum in the FD at neutrino energies of $\sim 1-2 \mathrm{GeV}$. As such the neutrino enegies are mostly below threshold for $\tau$ production $(3.5 \mathrm{GeV})$ and the corresponding appearance search has not yet been performed.

Figures 4 and 5 from chapter 1 showed the latest results from MINOS [11]. Figure 29 below shows estimates for the sensitivity of MINOS to the atmospheric neutrino oscillation parameters using larger exposures of POT as well as indicating how the more exotic disappearance models of neutrino decoherence [55] and neutrino decay [56] can be ruled-out by MINOS data.
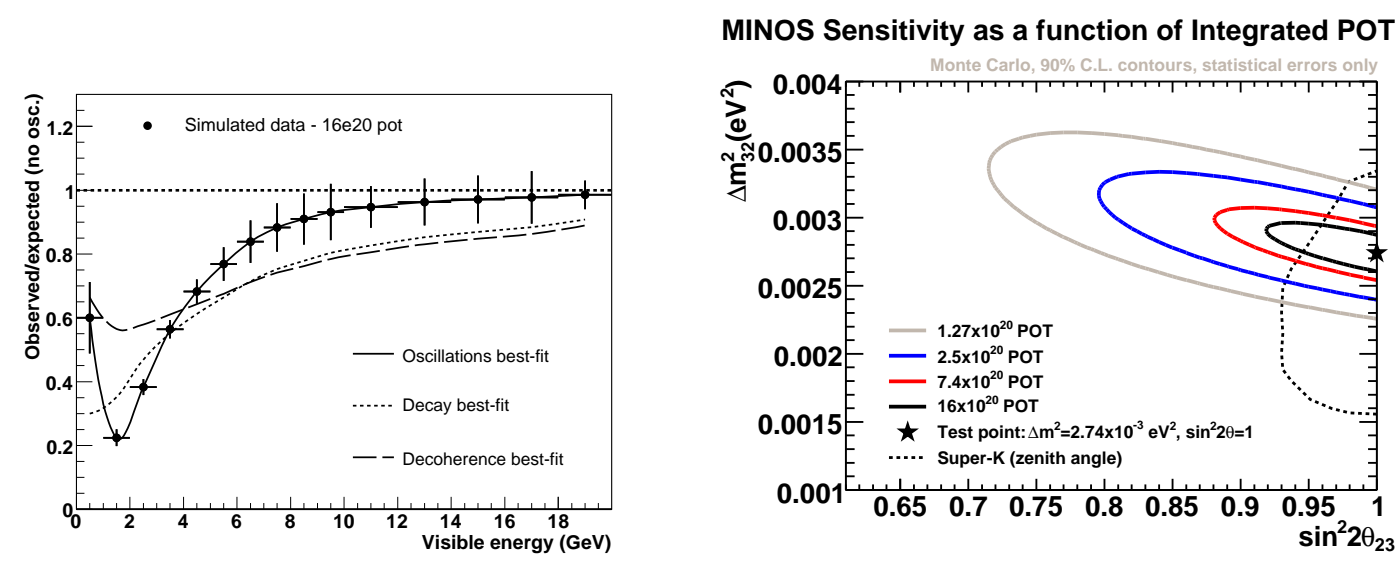

Figure 29: MINOS sensitivity to the atmospheric neutrino oscillation parameters based on a Monte Carlo simulation. The left-hand plot shows the ratio of simulated, oscillated data over the Monte Carlo expectation as a function of the reconstructed neutrino energy. The solid line shows the best fit to the oscillation hypothesis whereas the dotted and dashed lines show the best fits for the decay and decoherence hypotheses respectively. The right-hand plot shows the $90 \%$ confidence contours as a function of the oscillation parameters, $\Delta m_{32}^{2}$ and $\sin ^{2} \theta_{23}$, for a number of different POT exposures along with the results from the Super-Kamiokande experiment. As of the end of 2007 MINOS had collected $\sim 3 \times 10^{20}$ POT.

MINOS also hopes to be able to measure, or improve upon the existing limit of, the oscillation parameter $\theta_{13}$ which governs the sub-dominant oscillations of $v_{\mu} \rightarrow v_{e}$. This is a very difficult analysis both because the MINOS detectors were not designed to accurately reconstruct $v_{e}$ interactions and because the backgrounds, such as the $v_{e}$ contamination present in the NuMI 
beam, cannot be expected to be well modelled by Monte Carlo simulation.

This analysis constitutes an appearance search in the FD and figure 30 shows the MINOS sensitivity to $\sin ^{2} 2 \theta_{13}$ as a function of the CP-violating phase factor $\delta$. The current best limit on $\theta_{13}$ was set by the $\mathrm{CHOOZ}$ collaboration in 1999 [57] and if the neutrino mass hierarchy and values of $\delta$ and $\theta_{13}$ take certain values MINOS could exceed this limit or even discover $v_{\mu} \rightarrow v_{e}$ oscillations, given a large enough exposure of POT.

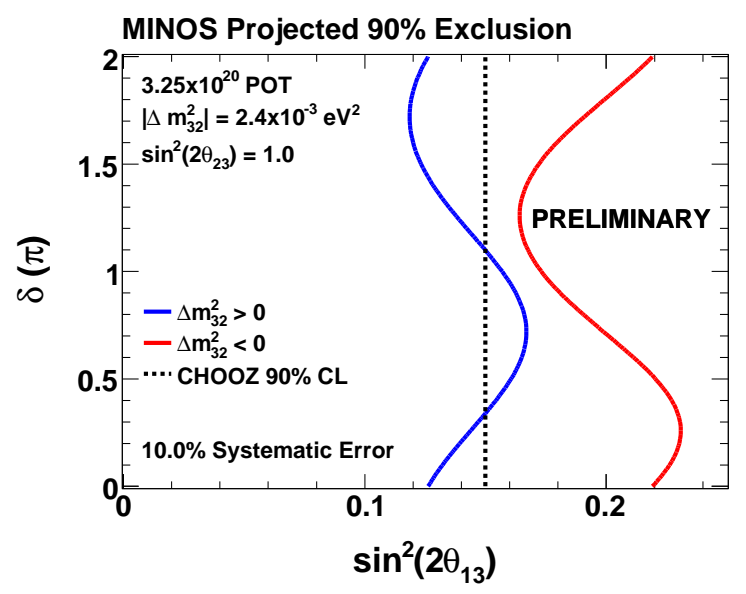

Figure 30: MINOS sensitivity to $\theta_{13}$ as a function of the CP-violating phase factor $\delta$ based on a Monte Carlo simulation. The dotted line shows the $90 \%$ confidence limit from the CHOOZ experiment whilst the red and blue curves show the MINOS sensitivity in either the normal or inverted neutrino mass eigenstate hierarchies. This plot also assumes the values of the atmospheric neutrino oscillation parameters, a total exposure of $3.25 \times 10^{20}$ POT and a $10 \%$ systematic error.

The second disappearance analysis that MINOS can pursue considers neutral current interactions which are sensitive to the presence of a sterile neutrino. Such a neutrino would have to have no Standard Model weak interactions otherwise it would have influenced the number of neutrino family measurements made by the LEP experiments at CERN using the width of the $Z^{0}$ boson [58].

A sterile neutrino has been proposed as an explanation for the results of the LSND experiment [59] which looked for $\bar{v}_{e}$ appearance in an $\bar{v}_{\mu}$ beam and found a neutrino mass splitting different from both the solar and atmospheric $\Delta m^{2}$ values (which would require four neutrino mass 
eigenstates). The LSND result was recently checked by the MiniBooNE collaboration [60] which did not find any evidence for neutrino oscillations in the region of parameter space suggested by LSND. Nevertheless the possibility of the existence of sterile neutrinos and neutrino oscillations corresponding to the LSND result remains an interesting topic in neutrino physics.

If $v_{\mu}$ neutrinos from the NuMI beam were oscillating into a sterile neutrino then MINOS would expect to see a disappearance at the FD in both the CC and NC energy spectra whereas if only the 'active' neutrinos were involved in oscillations then the FD NC energy spectrum would be unaffected by these oscillations. Figure 31 shows an illustration of oscillations to sterile neutrinos in MINOS.
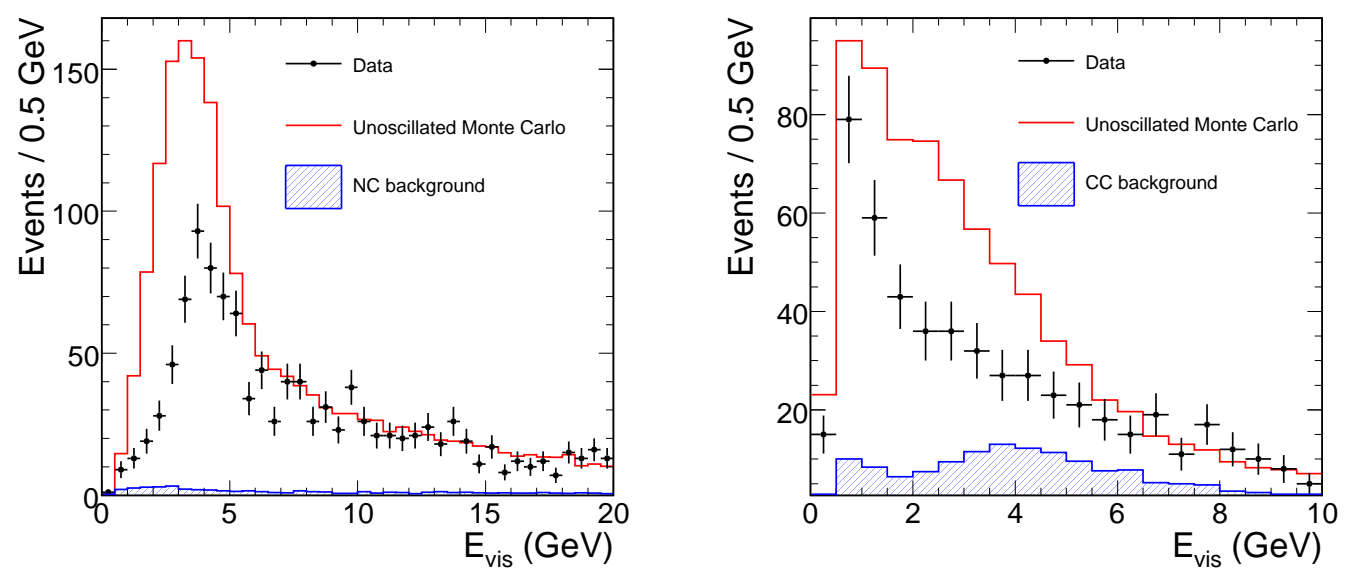

Figure 31: Example of oscillations into sterile neutrinos based on a Monte Carlo simulation. The lefthand plot shows the CC simulated data and MC along with the NC contamination in the sample whilst the right-hand plot shows the NC visible energy spectrum. This illustration assumes that $50 \%$ of the NuMI neutrinos oscillate to the sterile state.

MINOS can also use the large statistics data sample from the ND to perform non-oscillation neutrino physics analyses including measurements of the incident neutrino flux and neutrino interaction cross sections. Figure 32 shows the inclusive $\mathrm{CC}$ cross section as a function of neutrino energy, as measured by fifteen experiments, and it can be seen that the data points below $30 \mathrm{GeV}$ have large error bars. In the main running beam configuration for MINOS, the L010z185i beam, 70\% of the neutrino interactions are below $10 \mathrm{GeV}$ and so MINOS can make significant contributions to the knowledge of the inclusive CC cross section. 


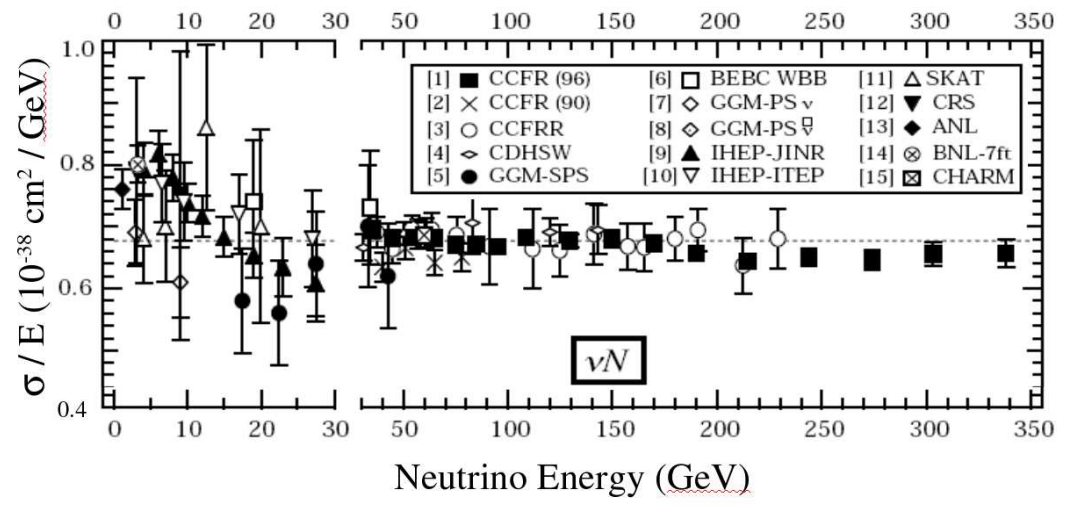

Figure 32: Inclusive $C C \mathrm{v}+N$ scattering cross section divided by neutrino energy as a function of neutrino energy as measured by fifteen experiments. The $x$-axis breaks at $30 \mathrm{GeV}$ above which the cross section is well constrained but below which the data points have large errors bars.

In addition to the inclusive measurements MINOS can look at specific channels. Deep-inelastic scattering (DIS) events can be used to extract the proton structure function $F_{2}\left(x, Q^{2}\right)$ as a function of $Q^{2}$ and figure 33 illustrates that MINOS can add data points in a new kinematic region. In the QEL scattering channel the work presented in this thesis will form the basis of a measurement of the cross section in terms of the axial-vector mass, $M_{A}^{Q E L}$. 




Figure 33: MINOS sensitivy to the proton structure function $F_{2}\left(x, Q^{2}\right)$ as a function of $x$ and $Q^{2}$ based on a Monte Carlo simulation. The plot assumes an exposure of $3.7 \times 10^{20}$ POT and the simulated MINOS data points overlap with experiments such as CCFR and NuTeV as well as extending into a new kinematic region. 


\section{Near Detector Data and Monte Carlo Simulation}

This chapter will discuss the reconstruction, calibration and modelling of neutrino interactions in the MINOS detectors. The first section will briefly introduce the separation of candidate single neutrino events from each beam spill in the ND and the tracking and energy reconstruction of these candidates. The second section will detail the calibration of the MINOS detectors and the third section will demonstrate the stability of the fully reconstructed and calibrated event sample recorded by the ND. The final section will present the Monte Carlo simulation of neutrino interactions in the MINOS detectors.

\subsection{Event Reconstruction}

MINOS uses the level of energy deposition, topology and timing of scintillator strips through which particles have traversed (hits) to reconstruct neutrino interactions inside the detectors. At the FD there is typically only one (or in most cases zero) neutrino interaction in the detector for a single beam spill but at the ND there are many interactions per beam spill. The first step in the ND reconstruction is to seperate out the candidate single neutrino events using the timing and spatial seperation of the hits. Figure 34 shows a single ND beam spill, containing multiple neutrino events, which is 'sliced' to form single neutrino events.

The timing and topology of the hits in each slice are then used to reconstruct tracks, based on a Hough Transform, and showers, using a clustering algorithm. Figure 35 shows an example of a $v_{\mu}$-CC and an NC Monte Carlo simulated event in a single view of the detector.

The reconstructed tracks are used to estimate the particle energy via it's range, if it stops inside the detector, or via it's curvature, if it exits the detector. For the curvature measurement a Kalman Fitter technique is used to fit the track trajectory and takes into account effects such as multiple Coulomb scattering. The energy resolution for the reconstructed muon energy is $\sim 5 \%$ when measured from range and $\sim 10 \%$ when measured from curvature. The curvature measurement also provides an estimate of the sign of the charged particle.

Showers are reconstructed by forming clusters of hits that are localised in space and time. The 

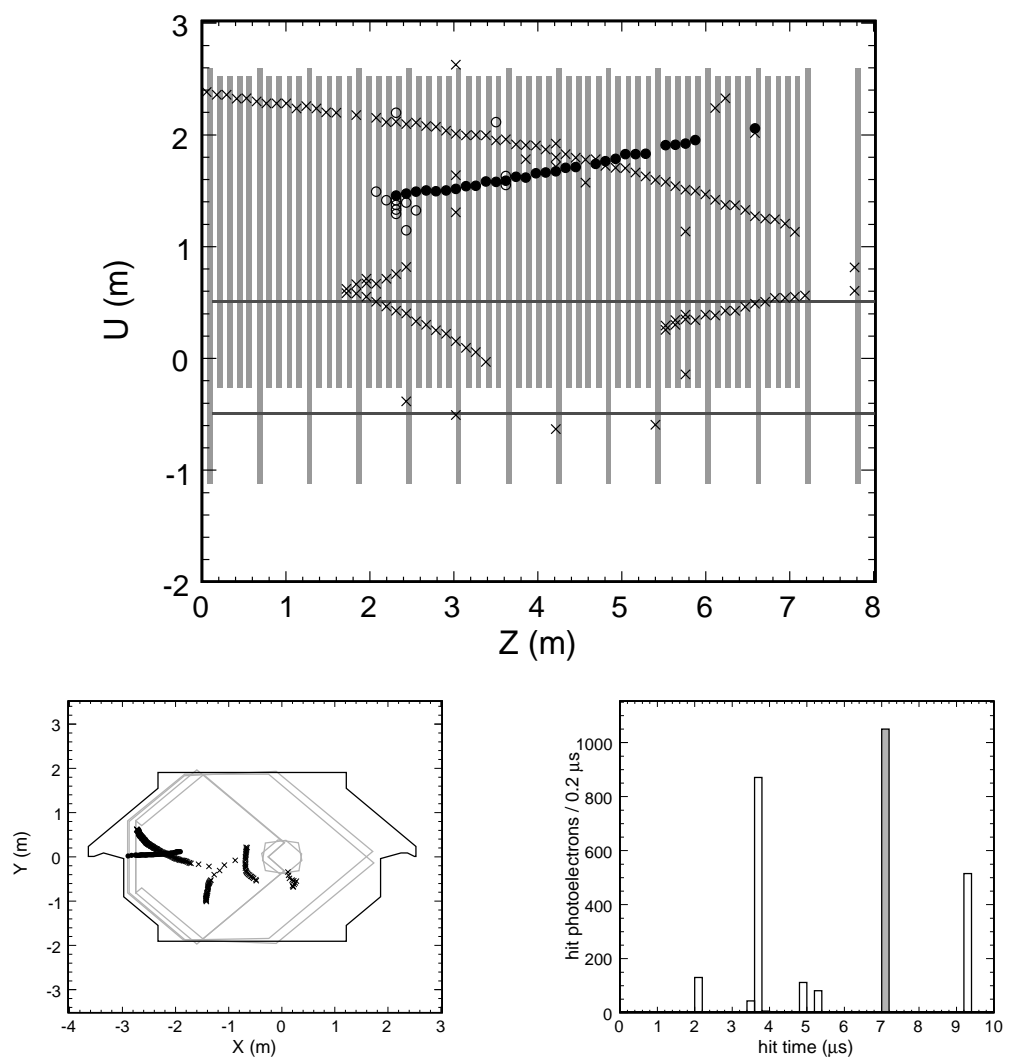

Figure 34: Activity in the ND during a single beam spill (a spill with less than the average 16 neutrino interactions has been chosen for clarity). The top figure shows one of the two possible orthogonal strip orientations (known as the $U$ and $V$ views). In the top figure; $\times$ denote hit strips and a single neutrino interaction has been selected with $\bullet$ denoting the reconstructed track hits and $\circ$ denoting reconstructed hadronic shower (and PMT cross talk) hits. The bottom-left figure shows a 'beam's eye view' of the spill along with an outline of the ND steel and grey outlines of the partially and fully instrumented regions. The bottom-right figure shows the time profile of the energy deposition in the detector with a shaded bin corresponding to energy deposition from the selected event. Figures taken from [11].

individual energy depositions of the hits in the cluster are then summed to provide an estimate of the energy of the shower. Often a hit can be reconstructed as part of both a track and a shower and in these cases the fraction of energy deposited that belongs to the track is removed (based on a minimum ionising particle assumption) and the remaining energy is attributed to the shower.

The reconstructed neutrino energy is then formed by summing the visible energy in an event coming from any reconstructed tracks and showers. The following chapter will further discuss 


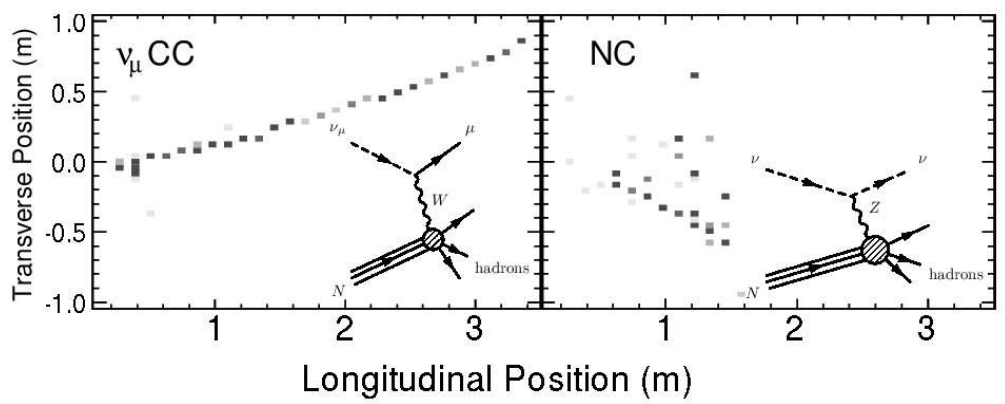

Figure 35: Two-dimensional views of a $\mathrm{v}_{\mu}$-CC and an NC event in the MINOS ND. The shaded rectangles show the hit strips with the darker shading corresponding to a larger energy deposition. The topology of the two event types is quite disimilar with the CC event dominated by a muon track, with some small amount of hadronic activity around the vertex, whilst the NC event consists of a diffuse shower. Figures taken from [11].

the selection of $v_{\mu}$-CC events in the ND as well as presenting the resolutions for a number of kinematic variables.

\subsection{Calibration of the MINOS Detectors}

The calibration of the MINOS detectors uses information from a special LED-based lightinjection (LI) system, test-bench scintillator measurements, measurements from CalDet and cosmic-ray muons in the detectors. The overall goal of the calibration procedure is to take a raw output signal $Q_{\text {raw }}(d, s, t, x)$, measured in detector $d$ by strip $s$ at time $t$ and at position $x$ along the strip, and convert it to a fully corrected signal $Q_{c o r}$. This is done via a number of 'calibration constants' that multiply the raw signal:

$$
Q_{\text {cor }}=Q_{\text {raw }} \times D(d, t) \times L\left(d, s, Q_{\text {raw }}\right) \times S(d, s) \times A(d, s, x) \times M(d)
$$

where the constants $D, L, S, A$ and $M$ correspond to the following corrections:

- Drift Correction: a time-dependant correction that accounts for changes in the response of the PMTs, readout electronics and scintillator. Originally this correction was perfomed using LI data to measure the individual strip gains but the variations between strips were found to be consistent enough for the drift calibration to be performed using 
the mean signal per plane induced by through-going cosmic-ray muons for each of the Near and Far detectors. Remaining differences between the strip outputs are corrected by the strip-to-strip correction mentioned below. Figure 36 shows that the $\%$ change in the detector responses over the data taking period used for the analysis presented in this thesis is $< \pm 1 \%$. A calibration constant for the drift in the two detectors is measured once per day.


Figure 36: \% change in the ND and FD response, in terms of mean signal per plane, to through-going cosmic-ray muons as a function of time. The variations are largely due to environmental considerations such as the temperatures in the ND and FD halls. Note that the zero point for the y-axes is arbitrary. Figure taken from [11].

- Linearity Correction: a correction that linearises the response of the PMTs and readout electronics as a function of deposited signal (also known as pulse height, PH). The LI system is used to produce 'gain curves' for each strip which map out the response as a function of PH. The linearity corrections are measured once per month.

- Strip-to-Strip Correction: a correction that normalises the individual strip responses to a mean strip response (in each detector seperately) by looking at cosmic-ray muons. This 'uniformity' correction accounts for differences in the light-output between different strips and uses the attenuation corrections mentioned below, an event-by-event 
accounting for the muon path length through the detector and also takes into account the expected inefficiency coming from the relatively low light-levels (the possibility that a muon does not leave any signal). The strip-to-strip calibration constants are measured once per month in the ND and once every three months in the FD and reduce the variation in strip responses from $\sim 30 \%$ to $\sim 8 \%$.

- Attenuation Correction: a procedure that corrects for the attenuation of the signal from each strip depending upon the position of the muon crossing along the strip's length. Prior to the installation of the detectors a test-bench setup involving a radiactive source was used to measure the signal output from each scintillator module at a number of different locations along each strip. The test-bench results were fit to a model of the optical attenuation along both the WLS fibres running along each strip and the clear optical fibres that route the light from the optical connectors to the PMTs. Figure 37 shows the effect of the various stages of the calibration upon the mean signal per scintillator strip per muon crossing. The attenuation corrections vary (on average) by factors of four along the $8 \mathrm{~m}$ FD strips and factors of 1.8 over the $3 \mathrm{~m}$ ND strips.

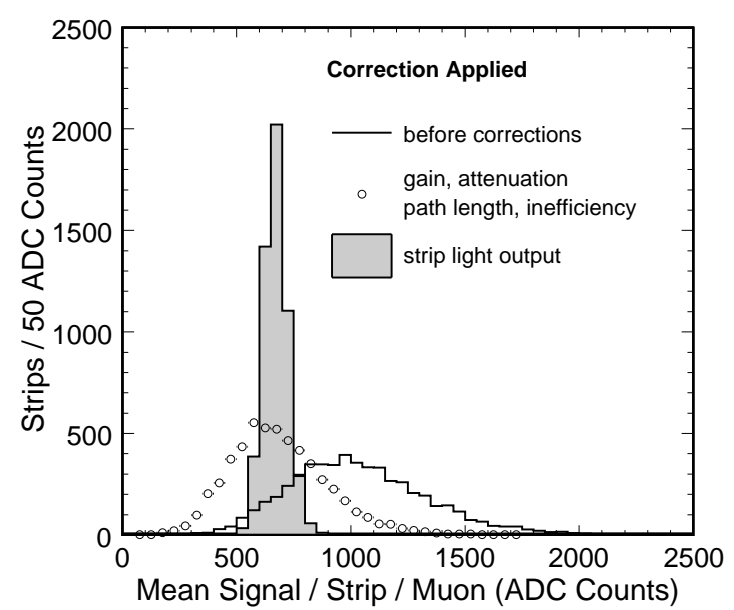

Figure 37: Effect of calibrations upon the mean signal per strip per muon crossing. The solid histogram shows the uncorrected data, the open circle points include the gain, attenuation, path length and inefficiency corrections and finally the shaded histogram shows the strip signal output after all of the corrections described up to this point. Figure taken from [11]. 
- Absolute Energy Scale: this final scale factor fixes the absolute energy scale in the detectors using the latter parts of the tracks of cosmic-ray muons that stop in the detectors [61]. At these latter parts of the tracks the muon energy loss is best understood and, having already been corrected by the above calibrations, the mean response is calculated for each strip with the average value from all the strips defining the 'muon equivalent unit' (MEU).

Now that the MEU has been defined the last calibration step is to set the MEU to GeV conversion factor for muons and neutrino-induced hadronic and electromagnetic showers. For muons comparisons of the corrected data from the Near, Far and Calibration detectors with the MINOS Monte Carlo simulation as well as information on muon stopping power from [62] yield the result that $1 \mathrm{MEU}$ corresponds to $2.00 \pm 0.02 \mathrm{MeV}$ of muon energy loss in scintillator. This calibration was performed independantly at both the ND and FD and the results agree to within $2 \%$.

Results from CalDet are also used to verify the electromagnetic and hadronic shower simulation performed using GEANT/GCALOR [63][64]. The measurements, an example of which are shown in figure 38, showed that the simulation agrees with the data at the level of $1-5 \%$ (depending upon the particle type). An energy dependant MEU to $\mathrm{GeV}$ conversion factor was extracted from the simulation such that the reconstructed shower energy estimates the energy transferred to the hadronic system.

\subsection{Near Detector Data Quality}

MINOS continually monitors the neutrino events being reconstructed in the ND which provide a check on the stability and quality of the neutino beam being produced by NuMI. Figure 39 shows that the number of reconstructed events per unit exposure in the ND is constant as a function of the run number across the data set used for the analysis presented in this thesis. The NuMI spill intensity varied over this set of runs, as the experiment moved on from the later stages of commissioning into regular beam operation, and figure 39 also shows that the number of reconstructed events in the ND is independant of the spill intensity. 


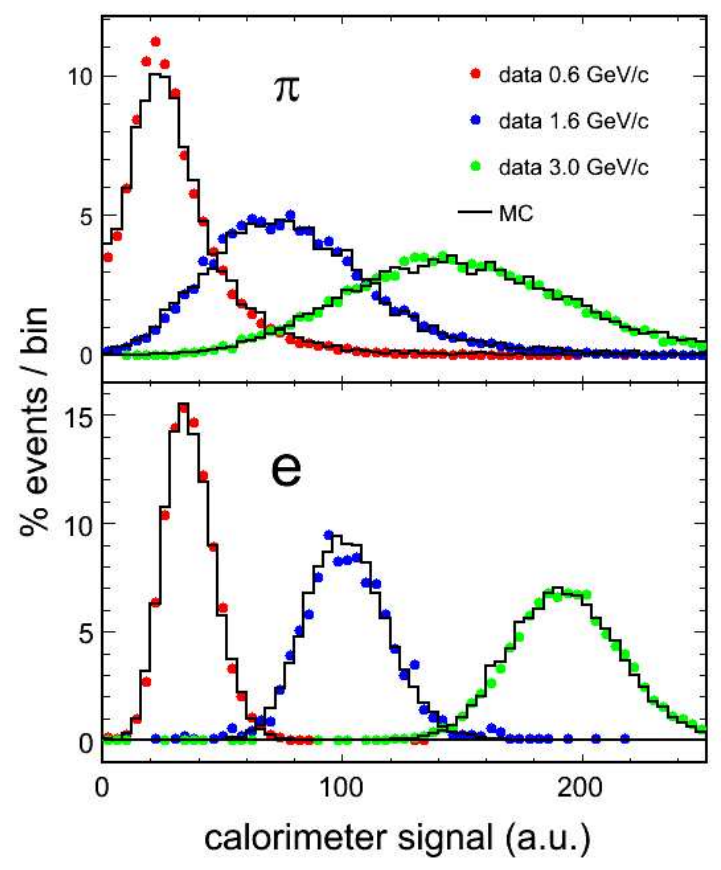

Figure 38: Detector response to the passage of electrons and pions of various energies. The coloured points show CalDet data and the solid histograms show the Monte Carlo simulation. The data agrees well with the simulation and it can be seen that for hadronic showers the width of the response curves is greater than that for the electromagnetic showers. The energy resolution for hadronic showers is $55 \% / \sqrt{E}$ and the energy resolution for electromagetic showers is 22\%/ $\sqrt{E}$ [63][64].

Figure 40 shows the mean reconstructed neutrino, muon and shower energies as a function of run number and shows that the ND event energy reconstruction is stable as a function of run number. This is important because the MINOS neutrino oscillation analysis compares the energy spectra of neutrinos at the Near and Far detectors. Figure 40 also shows the mean position of the reconstructed muon track vertex in the three cartesian coordinates as a function of run number and the flatness of these three sets of points confirms that the neutrino beam incident on the ND is focussed towards the same region as a function of time. Figure 41 confirms the stability of the NuMI neutrino energy spectrum and reconstruction and shows the reconstructed neutrino energy spectrum broken down by month of data taking.

The NuMI beam is instrumented with twenty-four capacitative beam position monitors (BPMs), two toroids, ten retractable segmented foil secondary emission monitors (SEMs) and three 

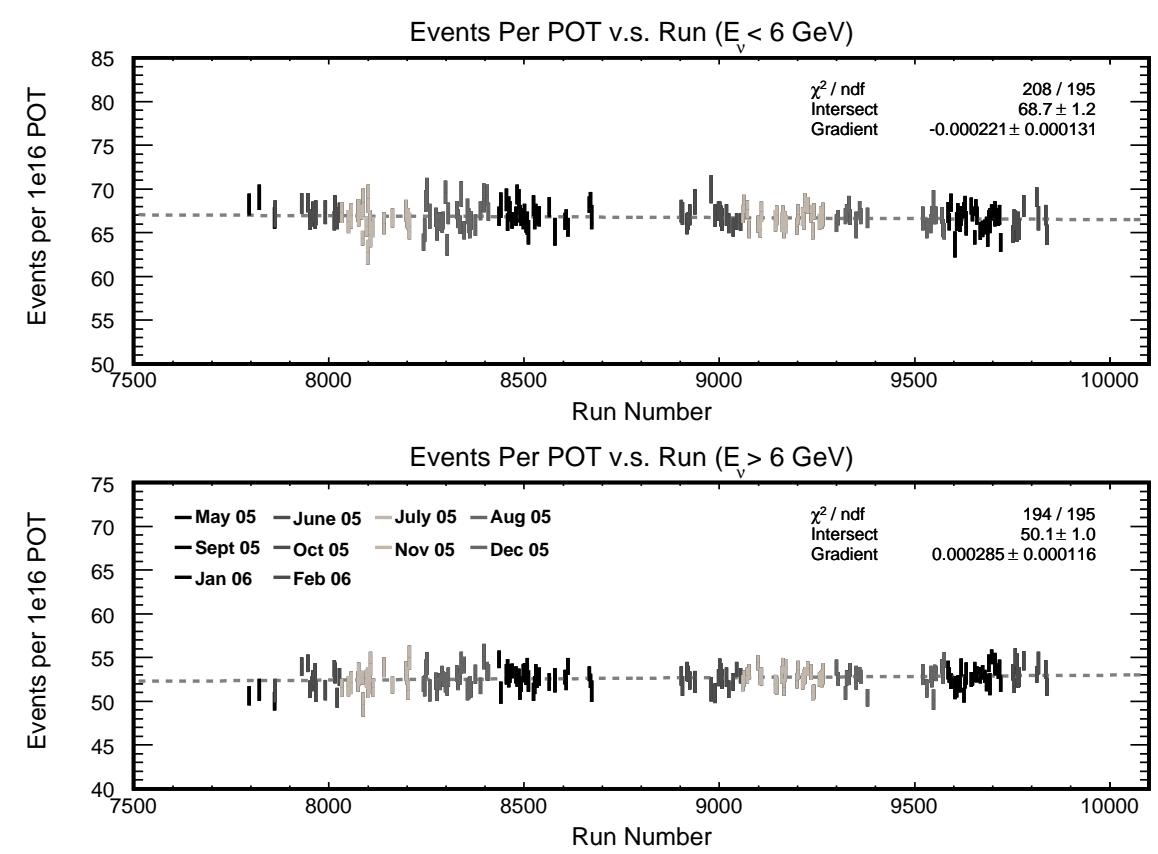

Figure 39: Number of reconstructed charged-current-like (CC-like) events per POT versus run number in the ND for data taking in the L010z185i beam configuration. The exact definition of CC-like will be presented in the following chapter. The top part of the figure corresponds to events with reconstructed energy below $6 \mathrm{GeV}$ and monitors the stability of the neutrino energy spectrum peak whereas the bottom part of the figure corresponds to events with reconstructed energy above $6 \mathrm{GeV}$ and monitors the stability of the high energy tail of the spectrum. The x-axis shows the MINOS run number and whilst usually a single run lasts 24 hours this is not always the case and so the $x$-axis does not quite have a linear correspondance with time. For presentational purposes runs with a total exposure of less than $2.5 \times 10^{17}$ POT are excluded from this figure. Such runs account for less than $10 \%$ of the overall total exposure. The dotted lines show fitted first order polynomials and illustrate that the event rate is very stable over this period of data taking.

muon stations (ionisation chambers) which allow measurements of the stability of the neutrino beam, it's position on the target and it's profile and halo. The ND data quality checks provide an extra layer of quality control and also serve to check the calibration and reconstruction of the NuMI neutrino events. 

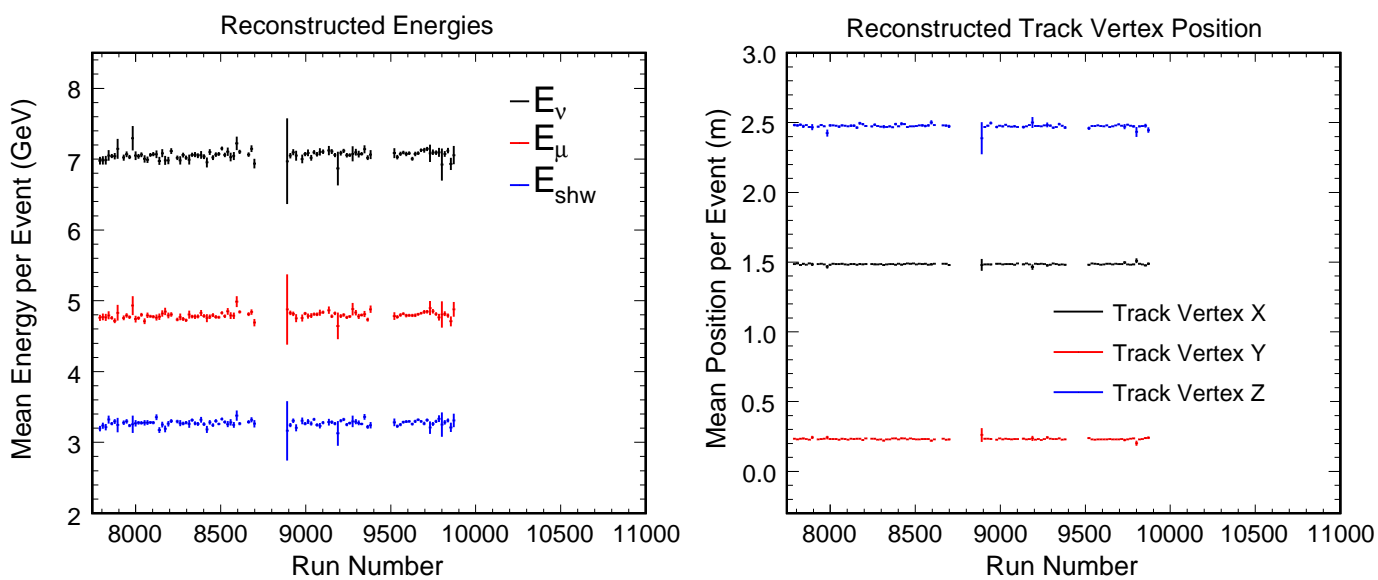

Figure 40: The left-hand plot shows the mean reconstructed neutrino, muon and shower energies for CC-like events in the L010z185i beam configuration as a function of run number. All three sets of points are consistent with straight lines verifying that the neutrino, muon and shower energies and their reconstruction are stable as a function of time. The right-hand plot shows the mean reconstructed muon track vertex position for CC-like events in the L010z185i beam configuration as a function of run number. These three sets of points show that the beam position at the ND and the track vertex reconstruction are stable as a function of time.

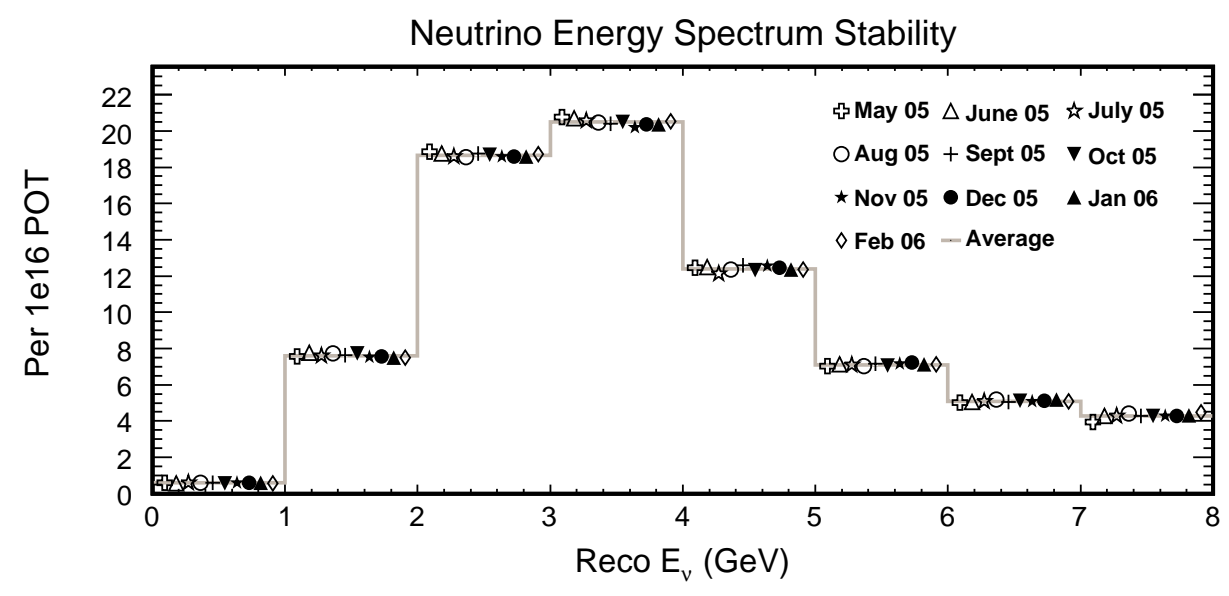

Figure 41: Reconstructed neutrino energy spectrum for CC-like events broken down by month of data taking in the L010z185i beam configuration. The gray histograms shows the average value over the months and each point shows the bin content for that month with the same binning as the average value histogram. The month-by-month neutrino energy spectra agree well with a largest deviation between months of $\sim 2-3 \%$. 


\subsection{MINOS Monte Carlo Simulation}

The MINOS Monte Carlo simulation (MC) consists of the simulation of hadron production off the NuMI target, the propogation of these hadrons and the resulting neutrinos to the Near and Far detectors, the simulation of neutrino interactions in the detectors and finally simulations of particles traversing the detectors and the detectors' response and readout.

The FLUKA 5 Monte Carlo simulation is used to calculate the production of secondary hadrons coming from the inside of the NuMI target as well as to model their passage through it. The simulation of the other components of the NuMI beamline, is performed in the GEANT-3 [65] based program GNUMI. Hadronic decays in which a neutrino is produced are recorded to be later used as input for the neutrino interactions and detector response simulations.

Neutrino-nucleus interactions in the MINOS detectors are modelled using the NEUGEN [66] event generator. NEUGEN includes descriptions of the most important neutrino interaction processes that occur in the MINOS detectors: quasi-elastic scattering, resonance production, deep-inelastic scattering and coherent pion production. Figure 42 shows the NEUGEN cross section predictions for a number of interaction modes along with data points from various experiments.

Quasi-elastic scattering is modelled, following the formalism presented in chapter 2, using BBA-2005 form factors and a dipole form for the axial-vector form factor. The value of

$F_{A}\left(Q^{2}=0\right)$ is set to -1.267 and the nominal value of $M_{A}^{Q E L}$ is $0.99 \mathrm{GeV}$. NEUGEN also uses a RFG model of the nucleus which includes the effects of Fermi motion and Pauli blocking.

NEUGEN uses a Rein-Seghal [67] treatment of resonance production and a Bodek-Yang [68] description of deep-inelastic scattering that has been extended [69] to improve the treatment of the transition region from resonance production to deep-inelastic scattering. At lower invariant masses ( $W<2.3 \mathrm{GeV}$ ) a modified $\mathrm{KNO}$ [70] scaling is used to describe the multiplicity of the final state but for higher invariant masses this hadronic shower model is integrated with PYTHIA/JETSET [71]. The transition from the KNO model to the PYTHIA/JETSET model takes place gradually between $2.3<W<3.0 \mathrm{GeV}$ above which the PYTHIA/JETSET model 


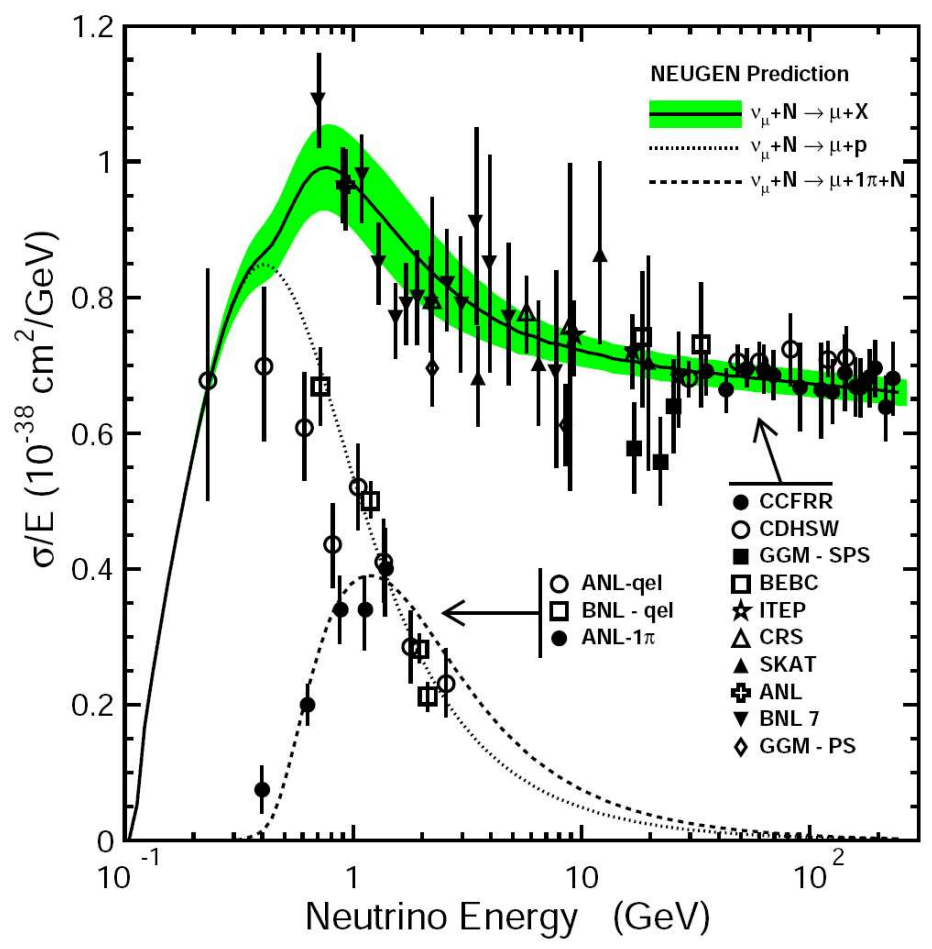

Figure 42: NEUGEN cross sections for inclusive $\mathrm{v}_{\mu}$-CC scattering, quasi-elastic scattering and single pion production as a function of neutrino energy. The cross sections assume an isoscalar averaged nucleon and the shaded band corresponds to the assumed uncertainty on the inclusive cross section. A systematic uncertainty of 3\% is assumed for the normalisation of the deep-inelastic scattering cross section $\left(W>1.7 \mathrm{GeV}^{2}\right)$ whilst $10 \%$ is assumed for the quasi-elastic scattering and resonance production cross sections.

is used exclusively. NEUGEN uses a modified version of the INTRANUKE [72] package to describe FSIs such as the re-absorption and re-scattering of final state pions and nucleons within the nucleus.

The modelling of the MINOS detectors uses the GEANT - 3 based program GMINOS. The simulation randomly samples the neutrinos saved from the FLUKA05/GNUMI output and traces them through the Near and Far detectors. Events are simulated both inside the detectors and in the surrounding rock and GMINOS is interfaced with NEUGEN to generate neutrino interactions. Particles produced by the neutrino interactions are transported in the detector by GMINOS. Hadronic interactions are simulated using the GCALOR [73] package which was benchmarked against measurements made at CalDet. 
Finally in the simulation the energy depositions are converted into light signals and then electronic signals in the PMTs and front-end electronics. These MC events are then presented to the MINOS reconstruction and analysis software in the same format as the real data. 


\section{Near Detector Event Selection}

This chapter will first describe the selection of a $v_{\mu}$-CC-enriched (CC-like) event sample in the ND. The resulting sample was observed to differ from the MC expectation in a way consistent with a mis-modelling of the NuMI neutrino beam. A reweighting procedure, using beam optics and target secondary hadron production parameters, is introduced and shown to improve the agreement between the ND data and MC. Next a procedure to select a $\mathrm{v}_{\mu}$-CC QEL-enriched (QEL-like) sample is described and comparisons of the QEL-like sample characteristics in data and $\mathrm{MC}$ are presented. Finally the MC is used to quantify the ND resolution for a number of kinematic variables.

\subsection{Selecting $v_{\mu}$-CC Events}

A number of criteria are imposed on the ND data and MC in order to define good $\nu_{\mu}$-CC events. For the data, checks are first made on the configuration and quality of the beam delivered by NuMI:

- Beam configuration: the $M_{A}^{Q E L}$ analysis presented in this thesis only considers data (and MC) from the L010z185i beam configuration. Data taken with the other beam configurations listed in table 5 is not considered in the extraction of $M_{A}^{Q E L}$ but is used for the $\mathrm{MC}$ tuning procedure that will be described in a coming sub-section.

- Coincidence with spill trigger: a cut is placed on the time a reconstructed event occured with respect to the nearest spill trigger. This cut removes the majority of the cosmic-ray muons that traverse the ND because the spill trigger window is short.

- Beam position on the target: the beam position on the target is a good measure of the stability of the beam being delivered by NuMI. The MC does not simulate neutrino interactions produced by an off-target beam (just the tails of the on-target beam) and so this cut also ensures that the $\mathrm{MC}$ and data samples originate from the same beam position on the target.

- Horizontal and vertical beam width: as with the previous cut the horizontal and vertical beam width is also a measure of the quality of the NuMI beam. The MC does not 
simulate an increased or reduced beam size and so this cut also ensures that the MC and data samples originate from a beam of the same horizontal and vertical width.

- Magnetic field: a check is made that the ND magnetic coil was operational. This cut ensures that the information on the curvature of particle tracks is available for analysis.

- Horn current: a check is made on the current flowing through the parabolic focussing horns. Different horn currents lead to different neutrino energy spectra at the ND and so this cut ensures that events used in the analysis come from the same underlying neutrino flux.

The beam quality checks listed above (excluding the selection of data taken with a particular beam configuration) remove approximately $1 \%$ of the data sample. Then the following CC-like sample pre-selection criteria are imposed on both the data and MC:

- Fiducial volume: reconstructed event vertices must lie within a $1 \mathrm{~m}$ diameter cylinder, centred about the neutrino beam spot and extending from $1 \mathrm{~m}$ to $5 \mathrm{~m}$ along the $\mathrm{z}$-axis. The diameter of the cylinder ensures that for most events the reconstructed hadronic shower is contained in the detector and also that charged particles entering through the sides of the detector are not accepted. The $z>1 \mathrm{~m}$ cut ensures that the analysis does not include events where the incident neutrino interacted in the rock upstream of the detector producing a muon that travels into the front face since in this case a measurement of the hadronic energy (and hence the neutrino energy) is not possible. The $z<5 \mathrm{~m}$ cut ensures that the hadronic showers from neutrino interactions are contained inside the calorimeter section of the ND allowing for a much more accurate estimate of the shower energy than is possible in the spectrometer region.

- Presence of a track: at least one track must have been reconstructed in the event. This cut removes a large portion of the NC events although without further cuts the remaining sample impurity from $\mathrm{NC}$ events where a track was reconstructed is $\sim 15 \%$. The text following this list of pre-selection cuts will describe the method used to further seperate $v_{\mu}-\mathrm{CC}$ and $\mathrm{NC}$ events. 
- Track reconstruction quality: the track finding algorithm quantifies the quality of the reconstructed track for a given event based on the reduced $\chi^{2}$ from the fit to the particle trajectory. This cut requires that the algorithm considered the track to be well reconstructed and also that the longitudinal difference in the track vertex position in the two orthogonal ( $\mathrm{U}$ and $\mathrm{V}$ ) views of the ND does not differ by more than six planes. This removes events where a bad fit to the track hits causes the the curvature estimation of the muon energy to be worse as well as events where a mis-reconstructed track vertex can cause both the curvature and range estimates of the muon energy to be worse. This cut also removes many $\mathrm{NC}$ events for which the reconstructed 'track' is really a collection of shower hits that have been mis-reconstructed.

- Track charge sign: the track fitter provides an estimate of the particle charge based on it's curvature in the magnetic field and this cut requires that the track corresponds to a negatively charged particle. This cut removes both $\mathrm{NC}$ events where the misreconstructed track happened to correspond to a positively charged particle and $\bar{v}_{\mu}$ interactions that produce a $\mu^{+}$.

The presence of a long muon track reconstructed in a MINOS neutrino event is a good indicator that the underlying interaction was $\mathrm{CC}$ but at low neutrino energies tracks are sometimes reconstructed in $\mathrm{NC}$ events; either spuriously or because of a pion ranging-out in the detectors and leaving hits that are truly consistent with a track. MINOS has developed [74] an event identification parameter to seperate $\mathrm{CC}$ (specifically $\mathrm{v}_{\mu}$-CC) from $\mathrm{NC}$ events beyond what is possible by simply requiring a reconstructed track.

The parameter is formed using a probability distribution function (PDF) based likelihood method. A number of 1-dimensional and 2-dimensional PDFs are contructed that show differences for true $v_{\mu}$-CC and NC neutrino interactions. The PDFs are based on the following distributions:

- Number of planes in the reconstructed track: reconstructed tracks in NC events will mostly be shorter than the long tracks from muons as they are due to reconstruction failures or pions, which do not travel as far as muons in the detectors. This variable is essentially a measurement of the track energy. 
- Number of track-like planes: for most CC events the muon track extends further in the detector than the hadronic shower whereas for $\mathrm{NC}$ events the reconstructed track is most likely contained inside the hadronic shower (either due to mis-reconstruction or a track-like set of strip hits corresponding to a final state pion). This variable is defined as the number of track planes containing strips that belong only to the track and not to a reconstructed shower and as such can distinguish between CC and NC interactions.

- Goodness of track fit: the track finding algorithm, used to estimate the muon energy via it's curvature in the magnetic field, provides a percentage error on the momentum measurement which can be used as a measure of the goodness of the track fit. For CC events, which contain real muon tracks, a better fit and hence a smaller percentage error is expected as opposed to the tracks reconstructed in NC events which are either misreconstructed sets of hit strips or correspond to hadronic tracks.

- Track mean PH per plane: the mean pulse height (PH) for muon track planes should be strongly peaked, as for the majority of the track the muon can be considered to be a minimum ionising particle (MIP). The mean PH for NC track planes will be more spread out with hits corresponding to hadronic particles such as pions (which do not deposit a consistent level of energy in the detector).

- Reconstructed y: the reconstructed y (inelasticity) is defined as the ratio of the reconstructed shower energy to the reconstructed neutrino energy. For CC events the $\mathrm{y}$ distribution is approximately flat but for NC events the hadronic shower energy usually constitutes the majority of the neutrino energy and as such the reconstructed y for NC events will be peaked towards one.

- Track charge sign: for $v_{\mu}$-CC events the reconstructed charge sign should almost always equal -1 whereas for reconstructed tracks from NC events the charge sign could take either the positive or negative value. This represents the fact that NC 'tracks' could be due to pions of either charge or reconstruction failures.

Figure 43 shows the above 6 variables for $v_{\mu}$-CC events and NC events from the L010z185i beam configuration $\mathrm{CC}$-like data and $\mathrm{MC}$ samples in the ND. The MC sample shown in fig- 
ure 43 has been weighted according to the reweighting scheme that will be described in the following sub-section and agrees well with the data points.
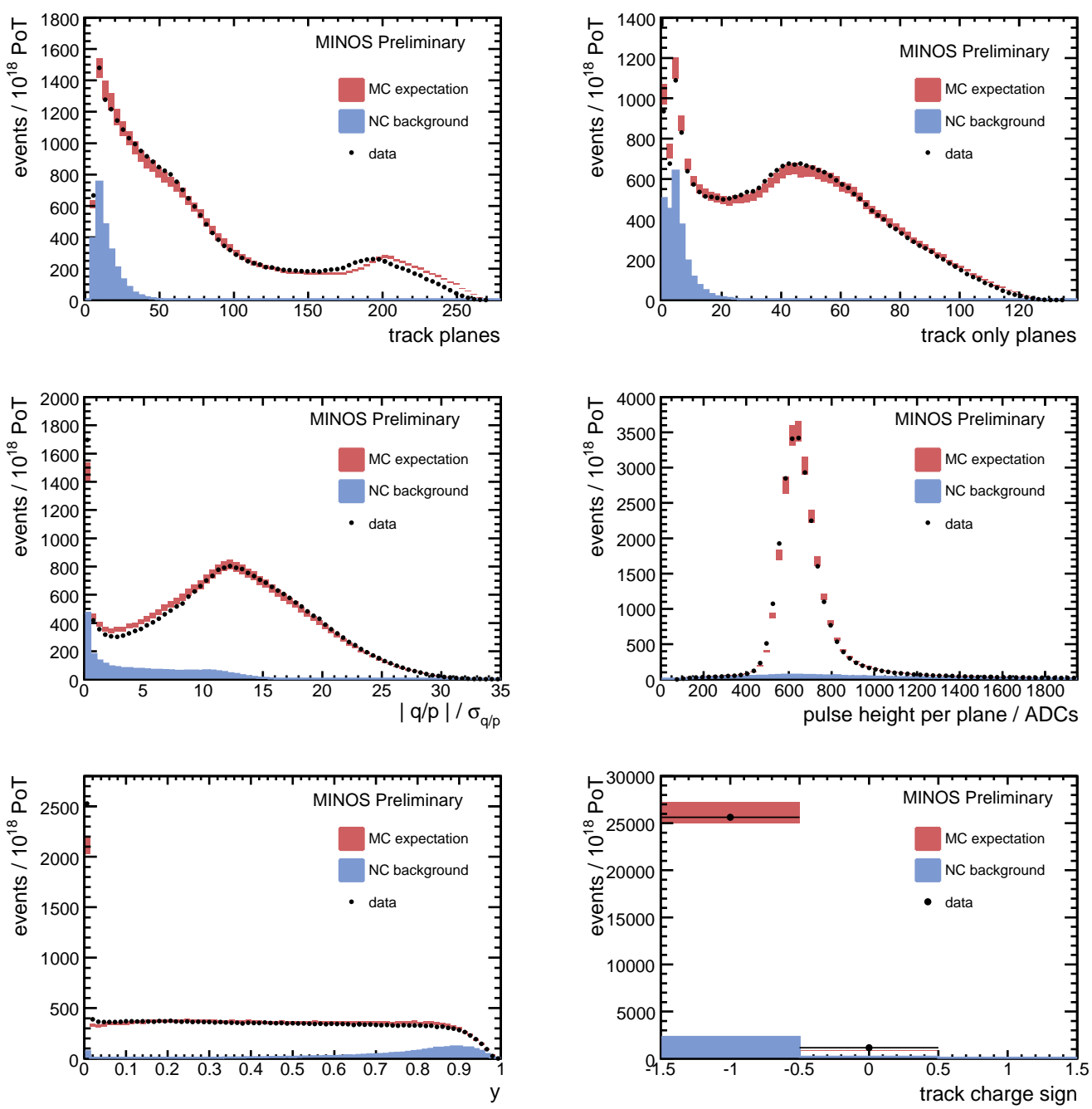

Figure 43: ND CC-like data and MC distributions of the event identification parameter input variables mentioned in the text. The black points show the data, the red histograms show the fully reweighted MC expectation for $\mathrm{v}_{\mu}$-CC events and the blue shaded histograms show the MC expectation for NC events. The red shaded envelope represents the uncertainties associated with the reweighting scheme that is described in the following section.

These distributions, when normalised to unity, represent the underlying PDFs for a given event to be either $\mathrm{CC}$ or $\mathrm{NC}$ as a function of the parameter values. For each event the compound probabilities for the event to be either $\mathrm{CC}$ or $\mathrm{NC}, P_{C C}$ and $P_{N C}$, are formed according to: 


$$
P_{C C, N C}=\prod_{i} f_{i}\left(x_{i}\right)_{C C, N C}
$$

where $f_{i}$ is the $i^{t h}$ PDF for either CC or NC events and $x_{i}$ represents either the one or two dimensions of this PDF. The event identification parameter, PID, is then defined according to:

$$
P I D=\frac{P_{C C}}{P_{C C}+P_{N C}}
$$

and takes values close to unity for $\mathrm{CC}$ events and close to zero for NC events. Figure 44 shows that the L010z185i ND CC-like data and MC identifiaction parameter distributions agree well. The final cut used to define the CC-like sample is a cut on the CC/NC separation parameter of $P I D>0.85$.

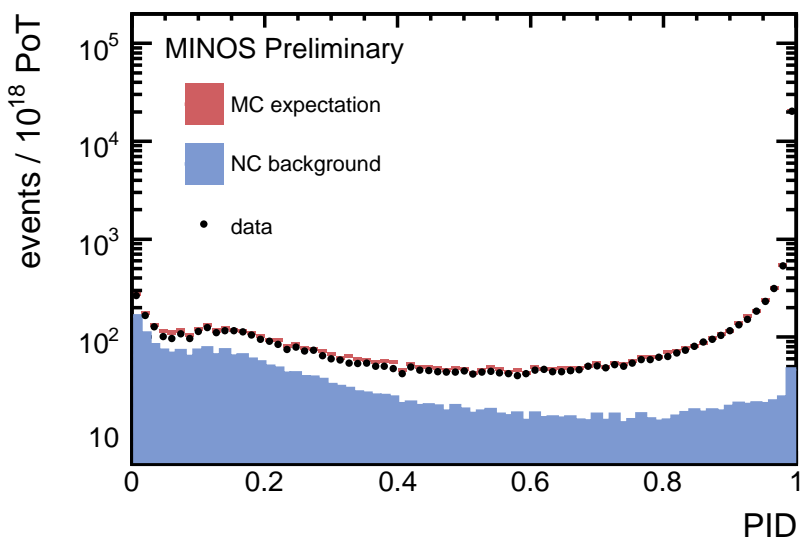

Figure 44: $C C / N C$ separation parameter for $C C$-like data and $M C$ in the $N D$. As with figure 43 the $M C$ has been fully reweighted according to the procedure described in the following section. The data and $M C$ distributions agree well, especially in the region where a cut is placed (>0.85) to select CC-like events.

Figure 45 shows the efficiency and purity (relative to the pre-selected sample) of this cut. The CC-like sample has high efficiencies and purities except at low reconstructed neutrino energies $\left(E_{v}<1 \mathrm{GeV}\right)$. In this low energy regime even the muon tracks from true $v_{\mu}$-CC interactions do not extend far and may be associated with large vertex hadronic showers causing the $\mathrm{CC}$ and $\mathrm{NC}$ separation to become more difficult. 


\begin{tabular}{|c|c|c|c|c|}
\hline Interaction Type & $\begin{array}{c}\text { Events per } \\
\text { 1e20 POT }\end{array}$ & $\begin{array}{c}\text { Percentage } \\
\text { of Total }\end{array}$ & $\begin{array}{c}\text { Events per } \\
1 \mathrm{e} 20 \mathrm{POT} \\
\left(E_{\mathrm{v}}<6 \mathrm{GeV}\right)\end{array}$ & $\begin{array}{c}\text { Percentage } \\
\text { of Total } \\
\left(E_{\mathrm{v}}<6 \mathrm{GeV}\right)\end{array}$ \\
\hline \hline $\mathrm{v}_{\mu}$-CC QEL & 333089.2 & 17.99 & 290399.6 & 25.24 \\
\hline $\mathrm{v}_{\mu}$-CC RES & 480091.2 & 25.93 & 402442.4 & 34.98 \\
\hline $\mathrm{v}_{\mu}$-CC DIS & 998035.9 & 53.91 & 428310.2 & 37.23 \\
\hline $\mathrm{v}_{\mu}$-CC COH & 10672.4 & 0.58 & 7780.2 & 0.68 \\
\hline $\mathrm{v}_{\mu}$-NC & 20423.1 & 1.10 & 17114.0 & 1.49 \\
\hline$\overline{\mathrm{v}_{\mu}}$ & 7849.0 & 0.42 & 3811.8 & 0.33 \\
\hline
\end{tabular}

Table 6: Numbers of selected CC-like events per le20 POT in nominal MC broken down by true interaction type for reconstructed $E_{\mathrm{v}}<120 \mathrm{GeV}$ and $E_{\mathrm{v}}<6 \mathrm{GeV}$. The second set of columns correspond to the peak of the neutrino energy spectrum.
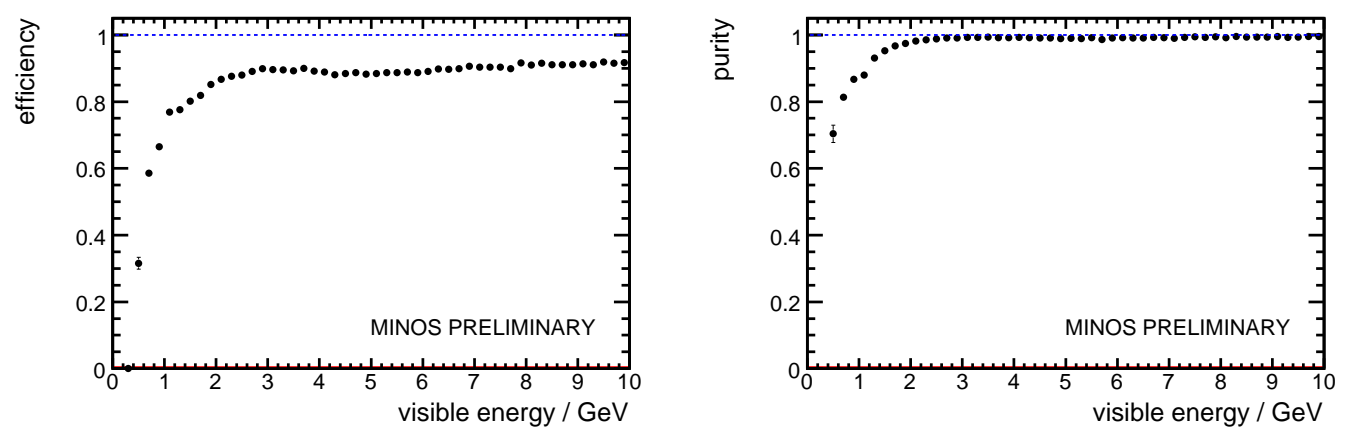

Figure 45: Efficiency and purity of the PID $>0.85$ cut as a function of reconstructed neutrino energy based on $M C$.

Table 6 shows a breakdown of the CC-like sample, in MC, by truth interaction channel in terms of the numbers of events per POT and the percentage contribution of that channel to the CC-like sample.

One of the main purposes of the MINOS ND is to provide a check on the simulation of the NuMI neutrino beam. The reconstructed neutrino energy spectra for ND data and MC in a number of different beam configurations are shown in figure 46. Figure 46 shows that the nominal MC does not agree well with the data and moreover that the disagreement changes as the beam configuration changes. This observation suggests that the difference between the data and MC is not due to effects such as cross section or detector mis-modelling (which would 
occur at a particular energy in all beam configurations) but rather due to a mis-modelling of the NuMI neutrino beamline.

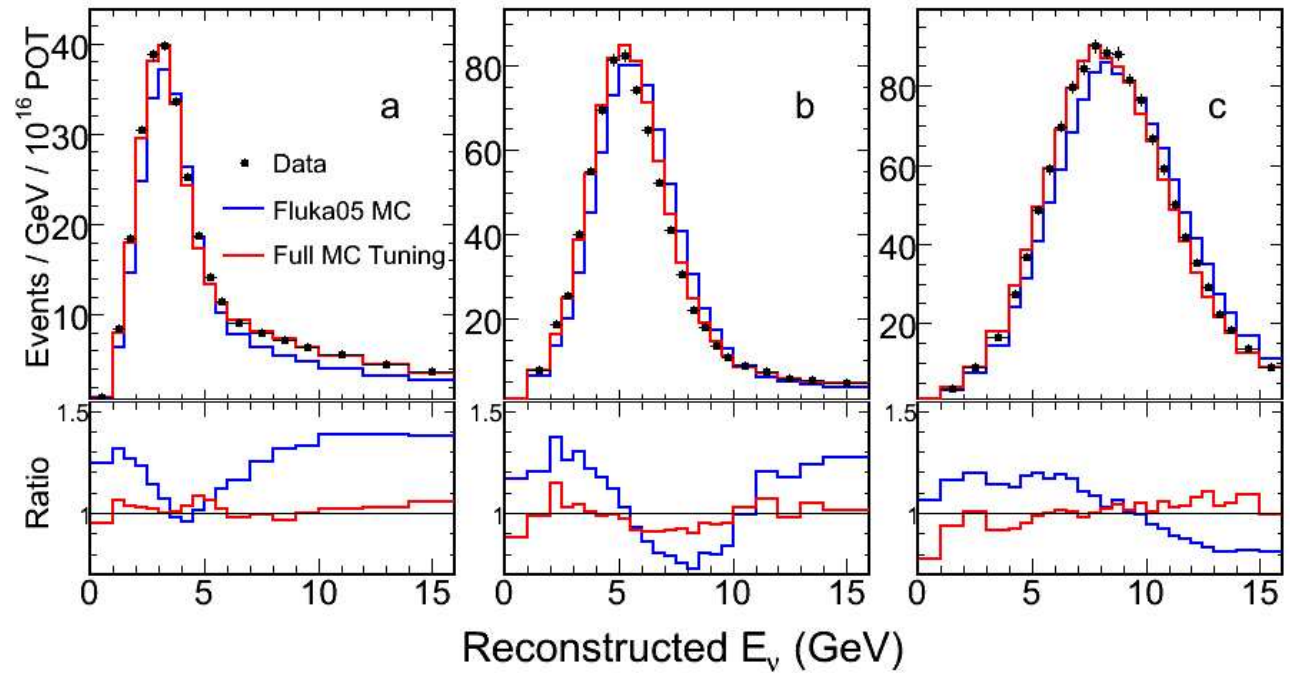

Figure 46: Comparison of ND data to nominal and fully reweighted MC in three different beam configurations; (a) the L010z185i beam, (b) the L100z200i beam and (c) the L250z200i beam configuration. The ratio figures exhibit a 'dip' structure that occurs on the falling edge of the neutrino energy spectrum in each of the different beam configurations.

\subsubsection{Beam and Hadron Production Parameter Reweighting}

The versitility of the NuMI beamline provides a powerful tool for understanding the neutrino flux incident on the ND. Figure 46 shows a data/MC disagreement that occurs at the falling edge of the neutrino energy spectrum in three different beam configurations and is suggestive of a mis-modelling of the NuMI beam. This disagreement is attributed to both a poor knowledge of secondary hadron production off the NuMI target and also from a number of beam focussing effects. A method has been developed by MINOS [75] to constrain the NuMI neutrino flux calculation using a fit to the reconstructed energy spectra of $v_{\mu}$-CC events in a number of different beam configurations.

The uncertainty in the modelling of hadron production from the NuMI target can be addressed because the relative positions of the target and first focussing horn and the horn current (which 
can be varied to yield the different beam configurations described in table 5) focus pions of different longitudinal and transverse momentum. Figure 47 shows the pion yields from the NuMI target in the six different beam configurations mentioned in table 5.
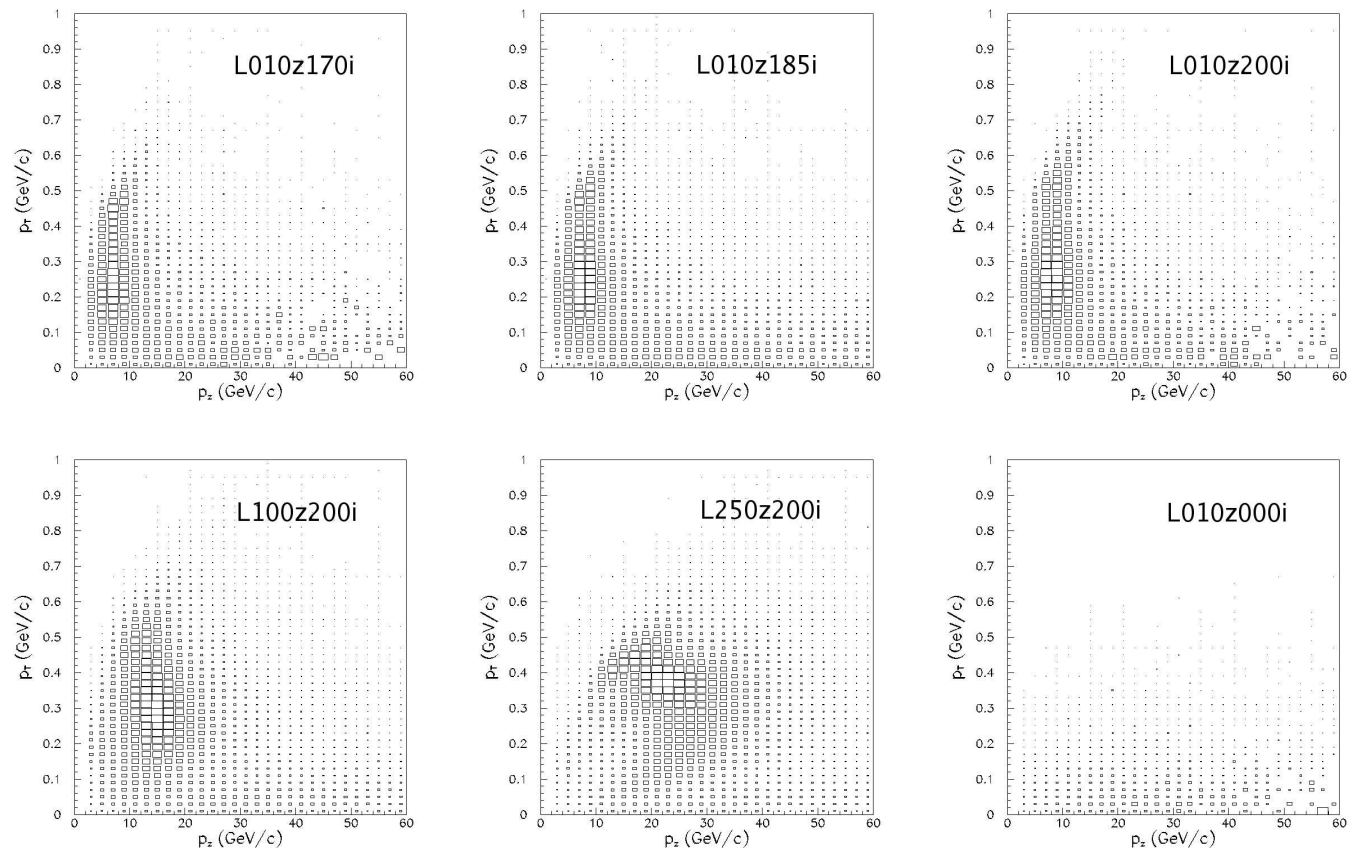

Figure 47: Pion yields from the NuMI target, as a function of pion $p_{z}$ and $p_{T}$, for six different beam configurations. The box sizes are proportional to the probability that a pion with the given $p_{z}$ and $p_{T}$ produced $a v_{\mu}$-CC interaction in the detector. Using CC-like energy spectra from the six beam configurations allows a sampling of $\left(p_{z}, p_{T}\right)$-space. Figure taken from [75].

The method first involves representing the FLUKA 05 target hadron production yield, $d^{2} N / d p_{z} d p_{T}$, as a parametric function. The parameterisation used is based on that originally proposed in [77]:

$$
\frac{d^{2} N}{d p_{z} d p_{T}}=\left[A\left(p_{z}\right)+B\left(p_{z}\right) p_{T}\right] \exp \left(-C\left(p_{z}\right) p_{T}^{3 / 2}\right)
$$

where the functions $A, B$ and $C$ can be written (in terms of $x_{F}=p_{z} / 120 \mathrm{GeV}$ ) as follows:

$$
A\left(x_{F}\right)=a_{1}\left(1-x_{F}\right)^{a_{2}}\left(1+a_{3} * x_{F}\right) x_{F}^{-a_{4}}
$$




$$
\begin{gathered}
B\left(x_{F}\right)=b_{1}\left(1-x_{F}\right)^{b_{2}}\left(1+b_{3} * x_{F}\right) x_{F}^{-b_{4}} \\
C\left(x_{F}\right)=\frac{c_{1}}{x_{F}^{c_{2}}}+c_{3}\left[x_{F}<0.22\right] \\
=c_{1} e^{c_{2}() x_{F}-c_{3}}+c_{4} x_{F}+c_{5}\left[x_{F}>0.22\right]
\end{gathered}
$$

The function $A$ can be thought of as determining the low $p_{T}$ yields whilst $B$ controls how fast the distribution rises with $p_{T}$ and $C$ influences the high $p_{T}$ fall-off. Figure 48 shows the fitted FLUKA05 yields as a function of $p_{T}$ and for a number of different values of $x_{F}$.



Figure 48: Parametric fits to the predicted target hadron production yields as given by FLUKA05. The black dots show the FLUKA 05 predictions, the black solid lines show overall fits using equation 76 and the solid red lines show the fitted yields using equations 77-79. Figure taken from [75].

Given the above parameterisation of the target hadron production yields, the MC energy spectra for the CC-like samples from the various beam configurations are fit to the data including 
a number of systematic parameters; modifications to the target hadron production yield, beam focussing/optics and detector-based parameters. Each of these sets of parameters will be briefly described below.

The parameters used in the fit to change the target hadron production yields are linear warpings in $x_{F}$ of the functions $A, B$ and $C$ :

$$
A^{\prime}=A\left(p_{0}+p_{1} x_{F}\right) \quad B^{\prime}=B\left(p_{2}+p_{3} x_{F}\right) \quad C^{\prime}=C\left(p_{4}+p_{5} x_{F}\right)
$$

where $p_{0}$ through $p_{5}$ are the fitted parameters. The weights used in the fits are constructed for positively charged hadrons $\left(\pi^{+}\right.$and $\left.K^{+}\right)$using a total of twelve parameters, $p_{0}$ through $p_{11}$, according to:

$$
W^{+}\left(\text {hadron type }, p_{T}, x_{F}\right)=\frac{\left[A^{\prime}\left(p_{z}\right)+B^{\prime}\left(p_{z}\right) p_{T}\right] \exp \left(-C^{\prime}\left(p_{z}\right) p_{T}^{3 / 2}\right)}{\left[A\left(p_{z}\right)+B\left(p_{z}\right) p_{T}\right] \exp \left(-C\left(p_{z}\right) p_{T}^{3 / 2}\right)}
$$

and for negatively charged hadrons the weights are tied to the weights for the positively charged hadrons using four parameters, $p_{12}$ through $p_{15}$, according to:

$$
W^{-}=W^{+}\left(p_{12}+p_{13} x_{F}\right)
$$

where only two parameters each are used to tune the target $\pi^{-}$and $K^{-}$yields because a much smaller fraction of anti-neutrinos (coming from the decay of negatively charged hadrons) are seen in the Near Detector CC-like sample.

The fits also consider a number of beam optics effects which relate to the uncertainties on the focussing provided by the horns, the error on the counting of the number of protons-on-target (POT) and the scraping of the beam on the collimator baffle. Figure 49 shows the effect of $1 \sigma$ changes to these parameters upon the true neutrino energy spectrum in the Near Detector.

The final set of parameters used in these fits are three detector-based parameters that are included to ensure that the fitted beam optics and hadron production parameters do not 'absorb' 


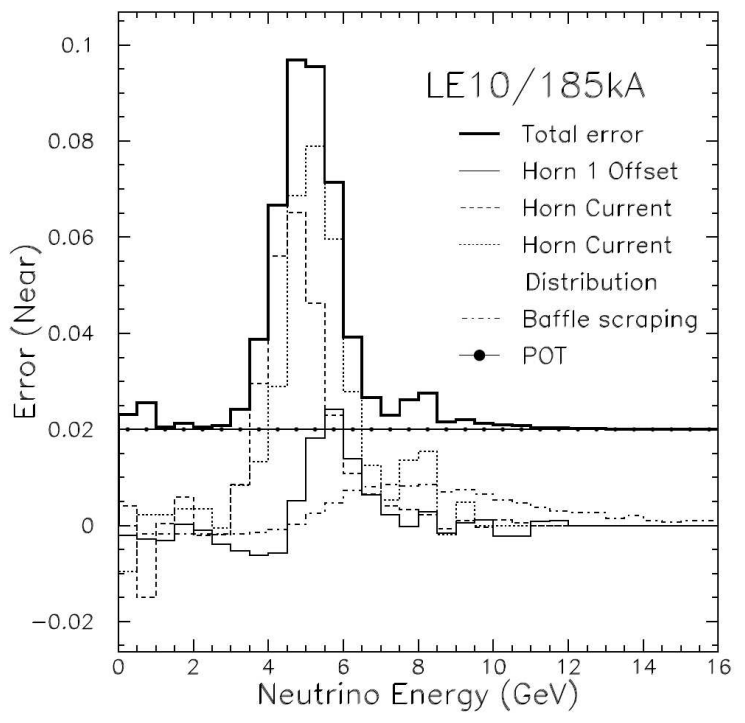

Figure 49: Errors on the ND neutrino energy spectrum coming from beam optics/focussing effects. The lines show the effects of $1 \sigma$ shifts due to an offset in the horn position, uncertainties in the current flowing through the horn's inner conductor (both it's absolute value and distribution), proton beam scraping on the collimator baffle and a $2 \%$ constant error on the absolute normalisation stemming from uncertainties in the counting of POT. Figure taken from [75].

a disagreement between data and MC that stems from uncertainties in the neutrino interaction cross sections and/or detector effects such as reconstruction and calibration uncertainties. Three parameters are included; a neutrino energy scale factor, a hadronic shower energy offset and a scaling factor for the true NC background events that are present in the CC-like sample. Similar MC flux tuning techniques have been employed by a number of previous experiments, such as [76].

In the results of these fits none of the fitted beam optics or physics parameters are shifted by more than their assumed $1 \sigma$ errors and the weights given to the parent hadrons are close to unity in the regions which give rise to neutrinos in the Near Detector (as shown in figure 50). Figure 46 shows that the fully reweighted MC agrees better with the data throughout including a large change in the high energy tail of the L010z185i beam configuration where the MC spectrum is shifted up by $20-30 \%$. The specifics of the fitting procedure and fit parameter constraints are discussed further in [11] and [75]. 


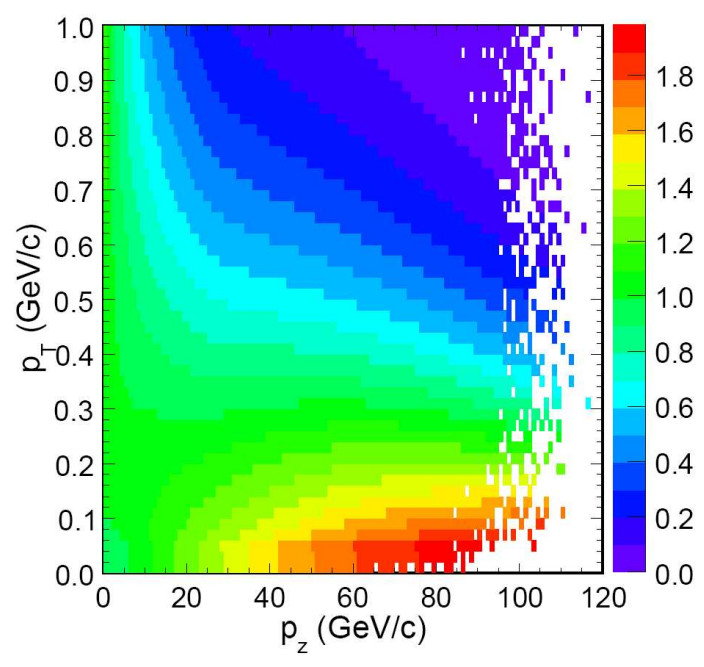

Figure 50: Scale factors for target-produced $\pi^{+}$as a function of $p_{z}$ and $p_{T}$. The L010z185i beam configuration is dominated by pions with average $p_{z} \simeq 10 \mathrm{GeV} / \mathrm{c}$ and average $p_{T} \simeq 0.3 \mathrm{GeV} / \mathrm{c}$ and in this regime the weights are close to unity. Figure taken from [75].

\subsubsection{Comparison of Data to Monte Carlo}

This section will present comparisons of the selected CC-like events in ND data and MC from the L010z185i beam configuration. The comparisons will make use of the MC reweighting procedure described above but will only apply the best fit values corresponding to the hadron production and beam optics parameters. The remaining detector response parameters were included in the above fits to ensure that the beam-related parameters did not absorb effects that originate from mis-modelling of the neutrino interaction cross sections or detector response but it is precisely these effects that the $M_{A}^{Q E L}$ analysis investigates. As such the following figures will show both nominal and 'tuned' MC with the label 'tuned' referring not to the full reweighting procdure (as was used in figures 43-46) but rather to a reweighting based solely on the best fit beam optics and hadron production parameters.

All of the distributions correspond to the samples of data and $\mathrm{MC}$ used in the $M_{A}^{Q E L}$ analysis, presented in the coming chapters of this thesis, with exposures of 1.2623e20 POT for the data and 9.4496e19 POT for the MC. The figures also show the dominant interaction types for the tuned MC, as selected using the MC truth information. These interaction types are $v_{\mu^{-}}$ $\mathrm{CC}$ quasi-elastic scattering (QEL), $v_{\mu}$-CC resonance production (RES), $v_{\mu}$-CC deep-inelastic 
scattering (DIS) and neutral current (NC) events. In general error bars are only shown for the data and tuned $\mathrm{MC}$ although the tuned MC errors are also indicative of the errors present for the nominal MC.

Figures 51 through 54 show some lower level reconstructed quantities; the reconstructed track vertex positions along the $x, y$ and $z$ directions and the reconstructed muon angle with respect to the incident neutrino beam. The shapes of the track vertex distributions are generally well modelled by the $\mathrm{MC}$ although there is a slight asymmetry between data and $\mathrm{MC}$ visible in the vertex $x$ distribution. The beam and hadron production tuning significantly improves the agreement between data and MC in all cases and the dominant effect here is the 20-30\% increase that was shown in figure 46 and that comes from increased weights for the target produced $\mathrm{K}^{+}$. The muon scattering angle shows reasonable agreement between the data and the tuned MC although there is evidence of a shift to higher angles in the data. The tuning process can been seen to be having the biggest effect at low muon scattering angles which corresponds to the higher neutrino energy events (that gain the largest weights from the reweighting procedure).
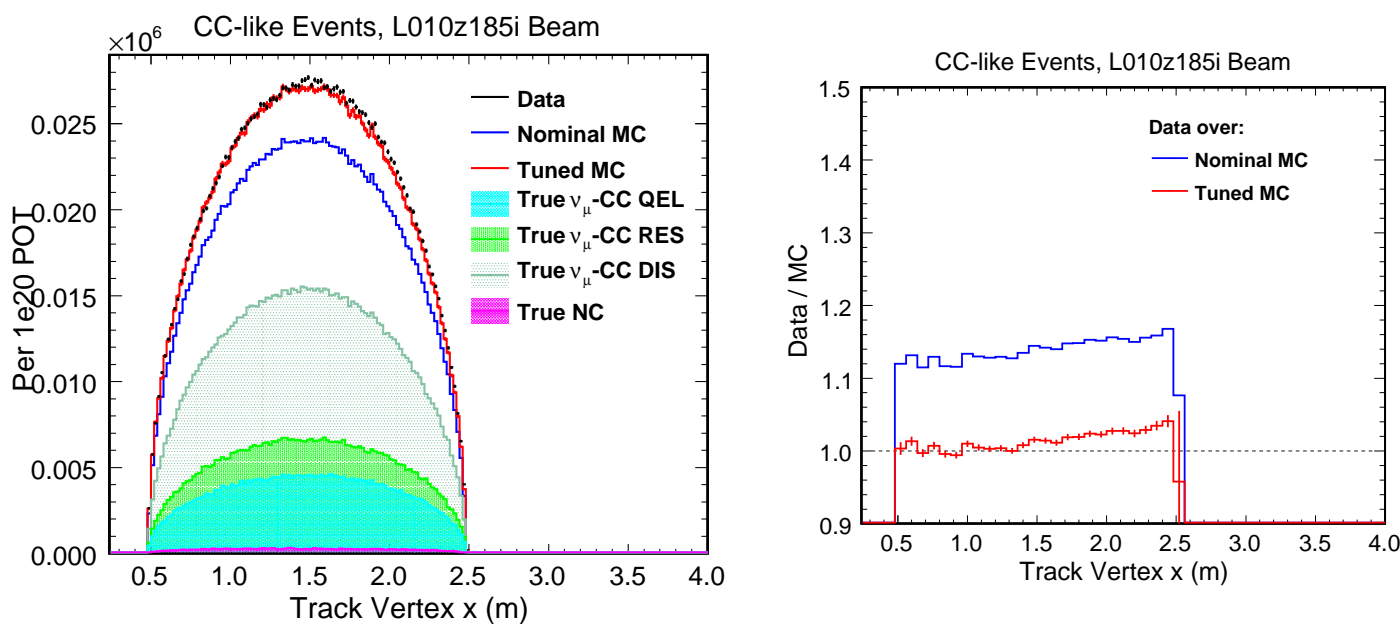

Figure 51: Reconstructed track vertex positions along the x-axis in data, nominal MC and tuned MC. The left-hand plot shows the distributions whilst the right-hand plot shows the ratios of data to the two MC distributions. 

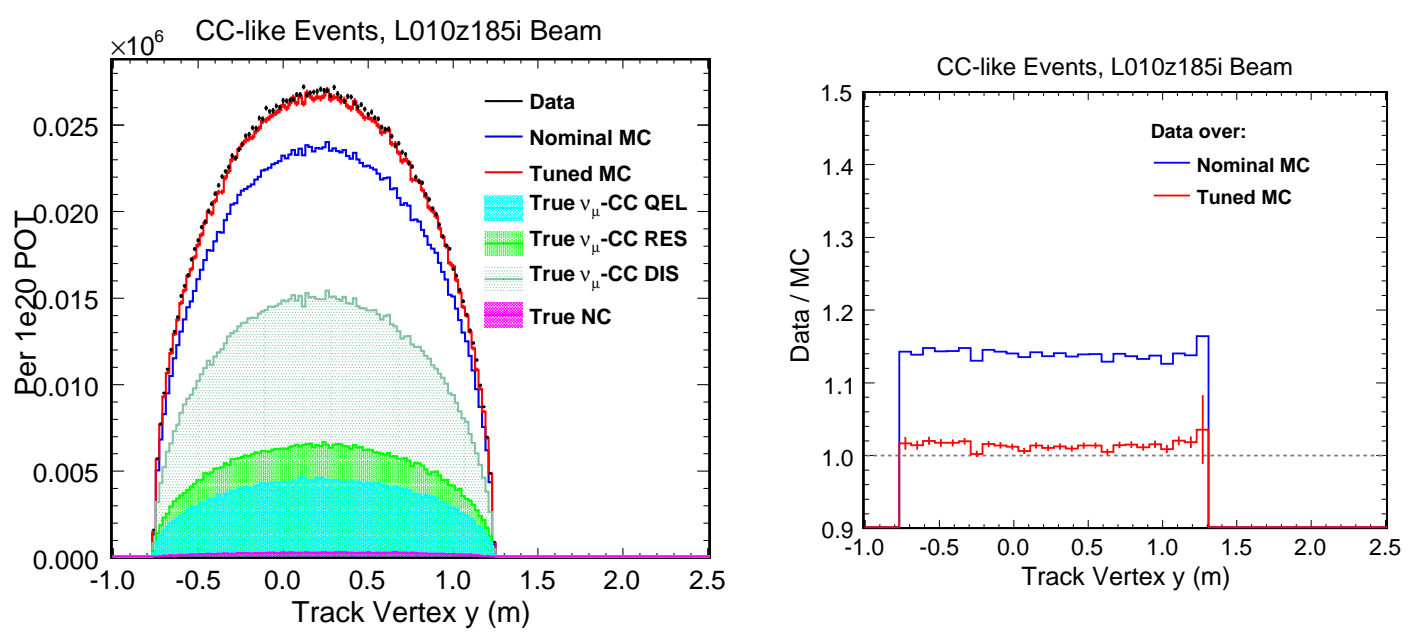

Figure 52: Reconstructed track vertex positions along the $y$-axis in data, nominal MC and tuned MC. The left-hand plot shows the distributions whilst the right-hand plot shows the ratios of data to the two MC distributions.
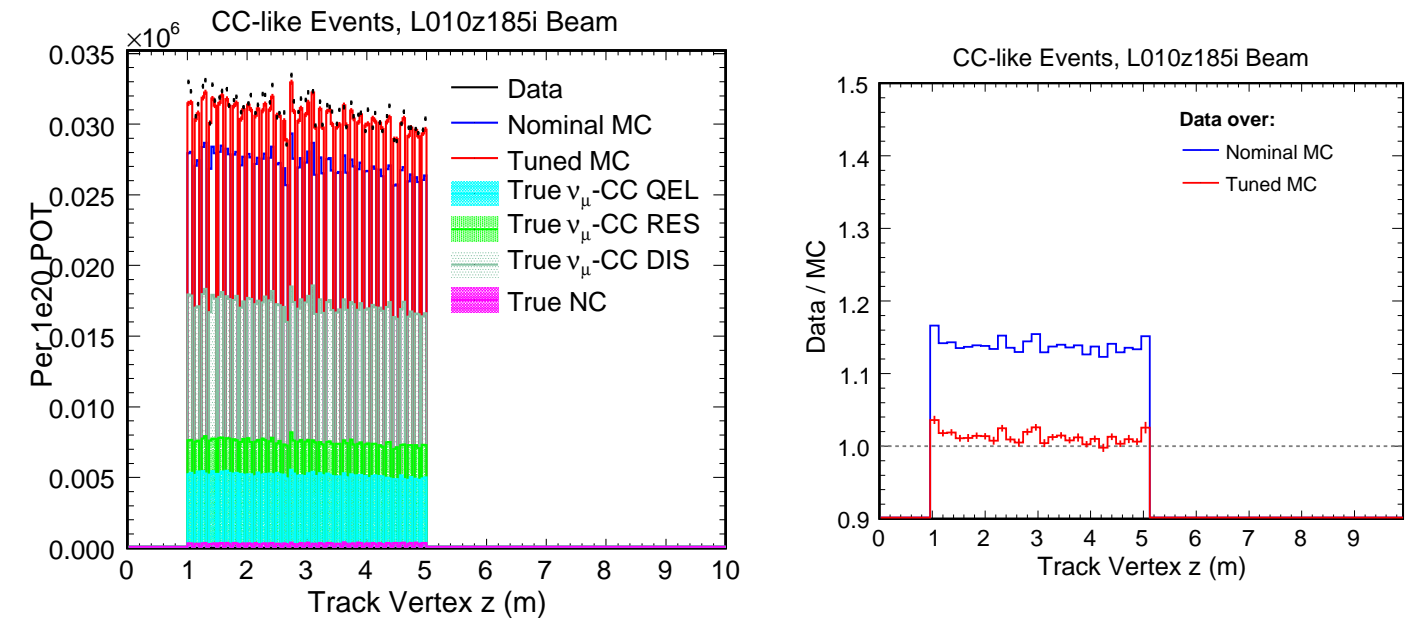

Figure 53: Reconstructed track vertex positions along the z-axis in data, nominal MC and tuned MC. The left-hand plot shows the distributions whilst the right-hand plot shows the ratios of data to the two MC distributions. 

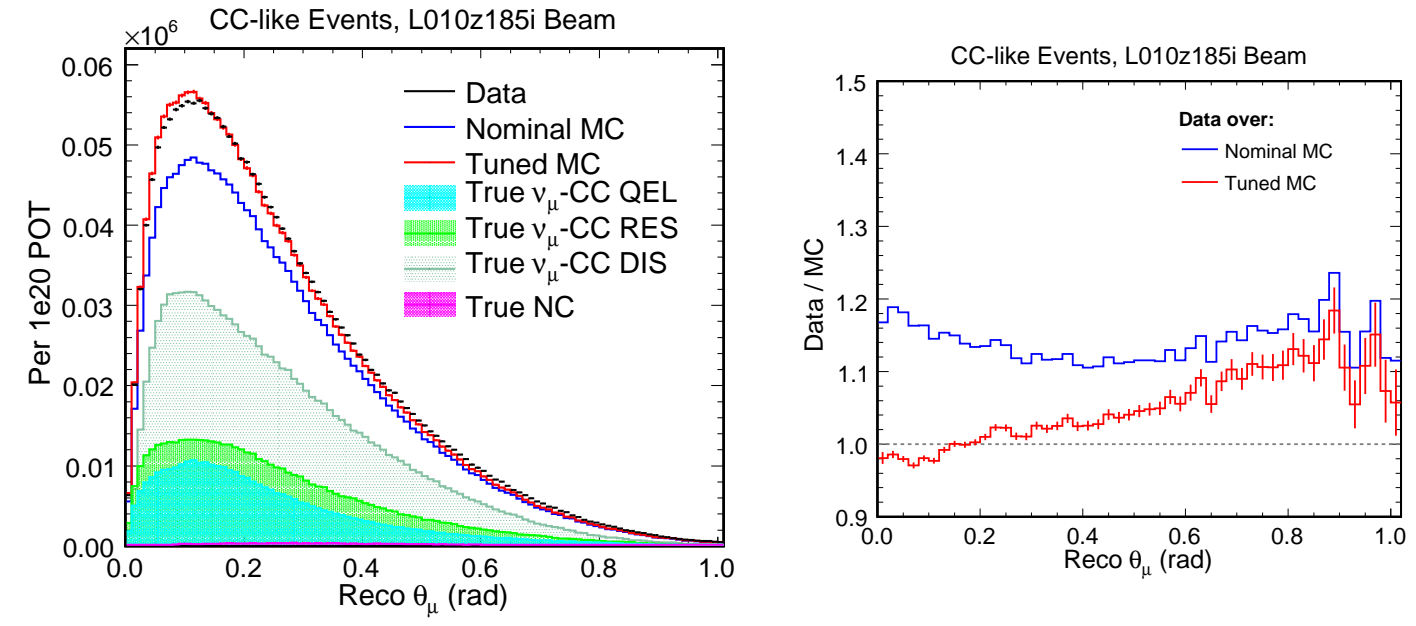

Figure 54: Reconstructed muon scattering angle with respect to the incident neutrino direction in data, nominal MC and tuned MC (left-hand figure) and ratios of data over MC (right-hand figure). 
Figures 55-59 show some higher level reconstructed quantities; the reconstructed muon and shower energies and the resulting reconstructed neutrino energy as well as the reconstructed $Q^{2}$ and invariant mass of the hadronic system. In all cases the tuning improves the agreement between data and MC although there is evidence throughout the figures of a deficit of events in the $\mathrm{MC}$ and differences in the shapes of the distributions between the data and MC. This MC deficit is prevalent in areas of the distributions where the QEL and RES interaction types dominate whereas where the DIS events are situated the data and MC agree better. This deficit is consistent with the uncertainties in the cross sections for these processes (see the caption of figure 42) with the higher energy and higher invariant mass DIS events being the best constained experimentally.
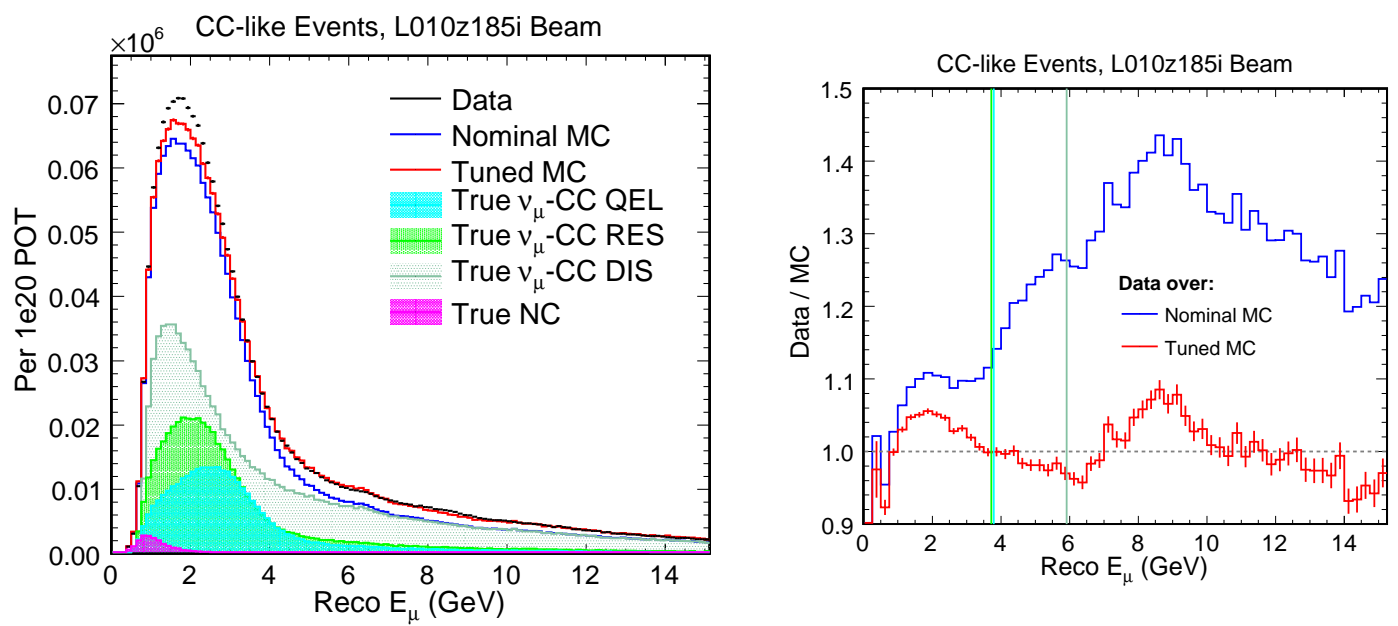

Figure 55: Reconstructed muon energy in data, nominal MC and tuned MC (left-hand figure) and ratios of data over MC (right-hand figure). The coloured vertical lines in the ratio figure show the mean values for true QEL, RES and DIS events (using the colour scheme of the legend in the left-hand figure) in the tuned MC and illustrate which interaction types dominate in the regions where the data and MC disagree. The mean for the QEL and RES events is very similar.

These data and MC comparisons show that, in general, there is a good level of agreement and that after the tuning process the MC is representing the data well. However, they are suggestive of possible cross section mis-modelling in the QEL and/or RES modes which further motivates the extraction of $M_{A}^{Q E L}$ presented in this thesis. 

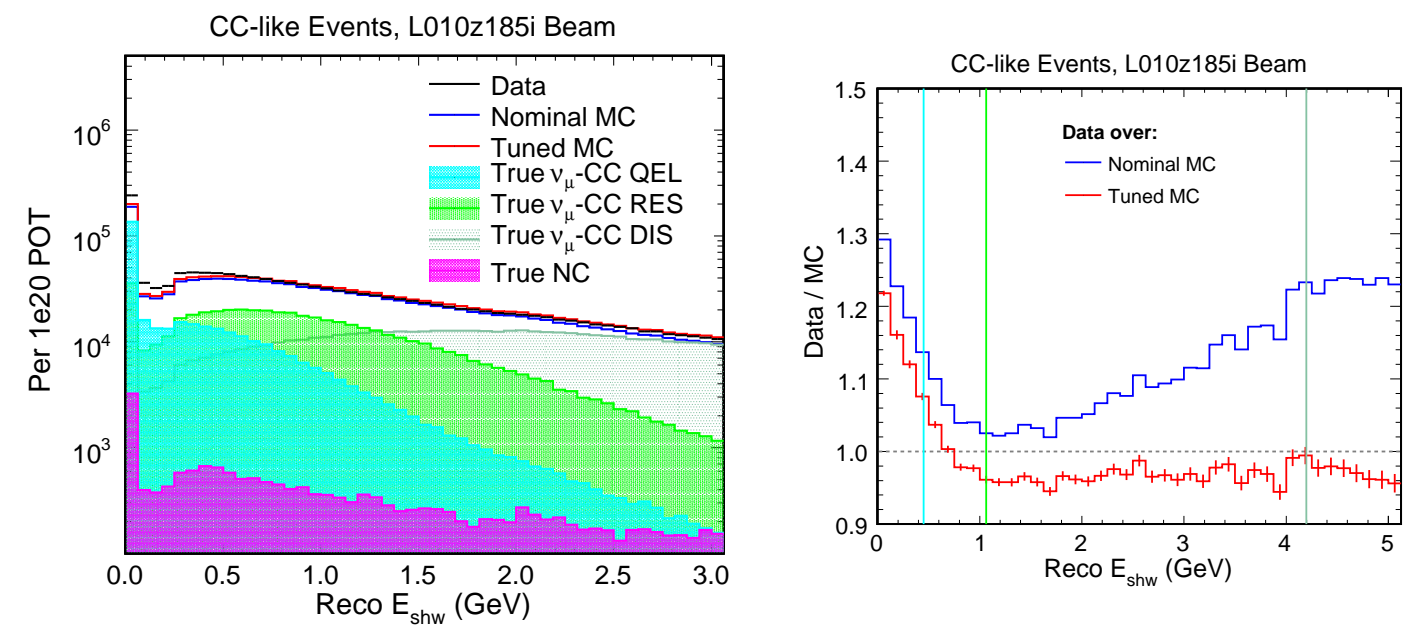

Figure 56: Reconstructed hadronic shower energy in data, nominal $M C$ and tuned $M C$ (left-hand figure) and ratios of data over MC (right-hand figure). The coloured vertical lines in the ratio figure show the mean values for true QEL, RES and DIS events (using the colour scheme of the legend in the left-hand figure) in the tuned MC and illustrate which interaction types dominate in the regions where the data and MC disagree. The x-axis has been extended in the right-hand figure to include the mean value for DIS events.
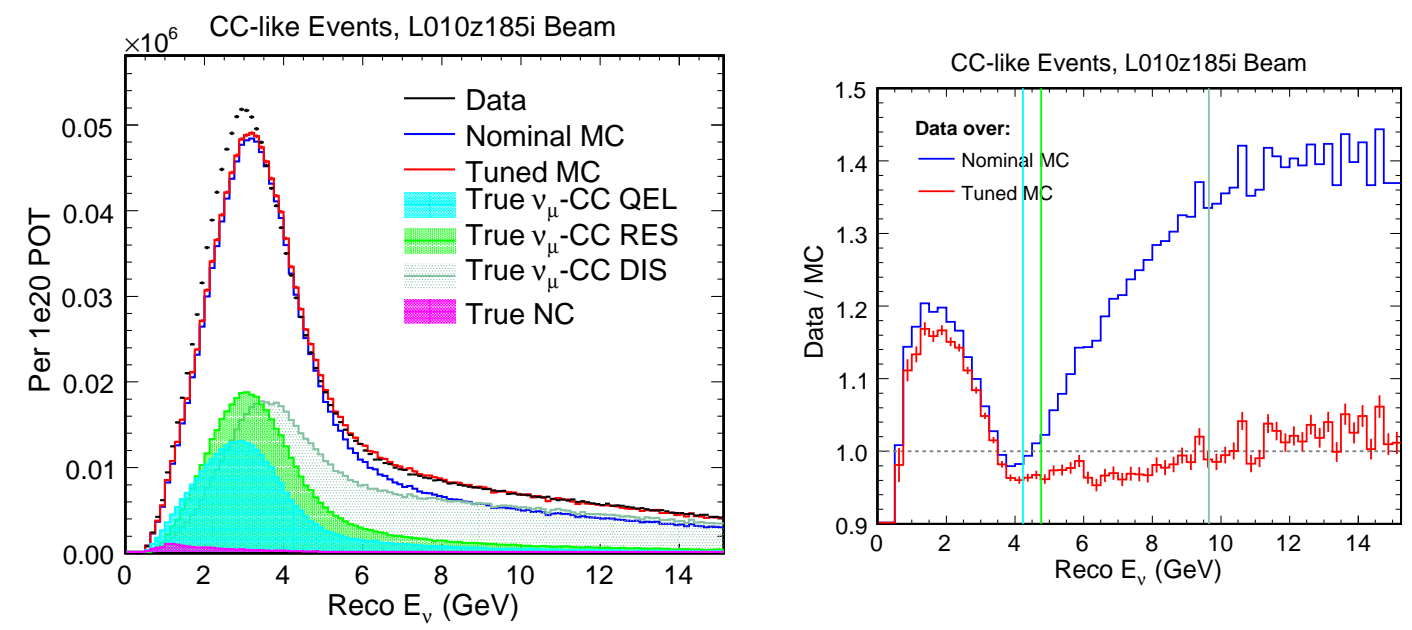

Figure 57: Reconstructed neutrino energy in data, nominal MC and tuned MC (left-hand figure) and ratios of data over MC (right-hand figure). The coloured vertical lines in the ratio figure show the mean values for true QEL, RES and DIS events (using the colour scheme of the legend in the left-hand figure) in the tuned MC and illustrate which interaction types dominate in the regions where the data and MC disagree. 



Figure 58: Reconstructed squared four-momentum transfer in data, nominal MC and tuned MC (lefthand figure) and ratios of data over MC (right-hand figure). The coloured vertical lines in the ratio figure show the mean values for true QEL, RES and DIS events (using the colour scheme of the legend in the left-hand figure) in the tuned MC and illustrate which interaction types dominate in the regions where the data and MC disagree.
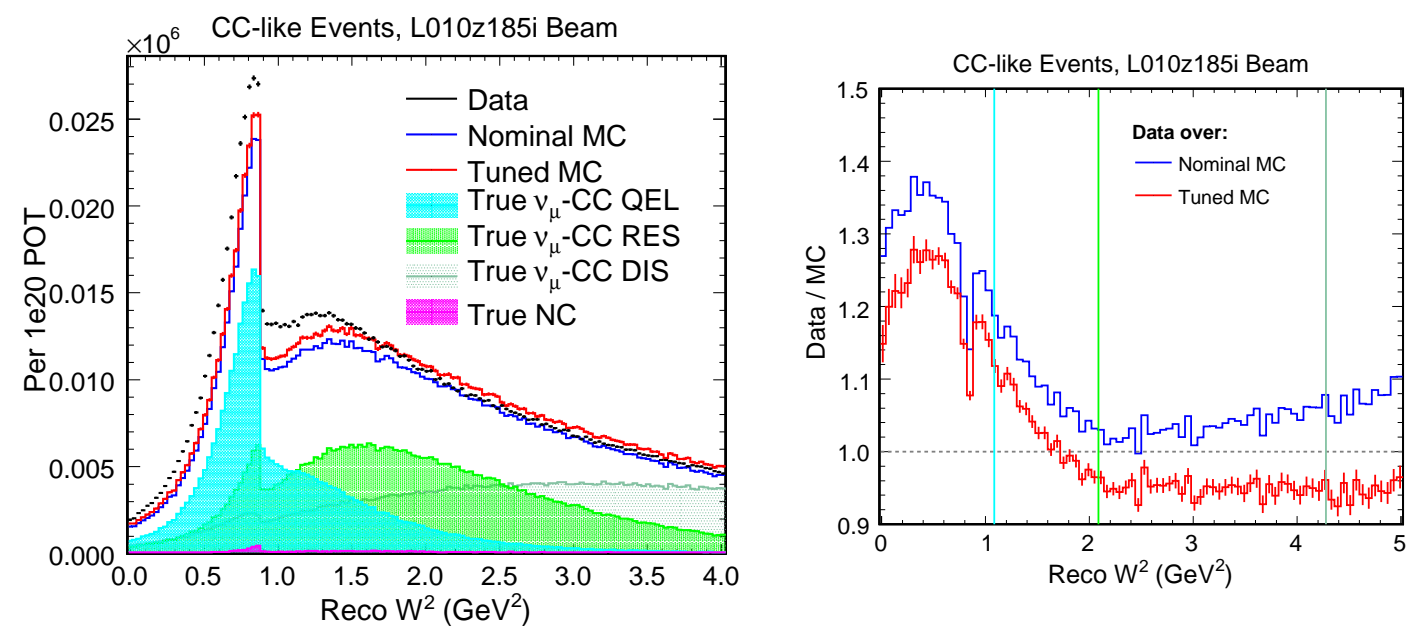

Figure 59: Reconstructed invariant mass squared of the hadronic system in data, nominal MC and tuned MC (left-hand figure) and ratios of data over MC (right-hand figure). The coloured vertical lines in the ratio figure show the mean values for true QEL, RES and DIS events (using the colour scheme of the legend in the left-hand figure) in the tuned $M C$ and illustrate which interaction types dominate in the regions where the data and $M C$ disagree. The x-axis has been extended in the right-hand figure to include the mean value for DIS events. 


\subsection{Selecting $v_{\mu}$-CC QEL Events}

A QEL-like sample is selected in data and MC by requiring two more criteria on top of the CC-like sample cuts:

- Reconstructed hadronic energy $<250 \mathrm{MeV}$ : the three major components of the CClike event sample are QEL, RES and DIS interactions. These interaction types could be defined (at the generator level) by considering the multiplicity of the hadronic final state with QEL events producing a single proton, RES events producing a proton and a pion and DIS events producing many pions. As such QEL events are likely to leave the smallest energy hadronic showers in the detector and figure 56 shows that below 250 MeV QEL interactions dominate the hadronic energy distribution.

- One and only one reconstructed track: the most likely interaction type in which two or more tracks could be reconstructed is DIS events with large hadronic showers. These showers can have spuriously reconstructed tracks traced through them or reconstructed tracks that correspond to pions that range-out in the detector. This cut does not remove many QEL events because for these events there is little hadronic activity (a single proton) around the vertex through which a track may be reconstructed.

The above QEL-enriched sample selection criteria are very simple and a number of more sophisticated methods have been tried:

- PFD-based likelihood: a likelihood method, using variables that show differences between QEL events and other interaction types as one-dimensional PDFs, was developed. These variables used the topology of the reconstructed vertex hadronic shower to discriminate between events and as such were taking advantage of the different hadron particle types and multiplicities found in the various interaction modes. This method was not used because it was found not to have a significant advantage over the simpler hadronic energy cut as well as requiring a large MC dataset for constructing PDFs and training the cut on the resulting identification parameter.

- Two-prong selection: an event selection has been developed [78] that removes the $E_{\text {had }}<250 \mathrm{MeV}$ events and concentrates on recovering QEL-like events from the re- 
maining sample. This technique attempts to identify the number of prongs (tracks) contained in an event and is able to separate QEL events, which have two prongs corresponding to the muon and proton, from RES and DIS events which have higher vertex hadronic shower particle multiplicites. This selection is complimentary to that gained by imposing the $E_{\text {had }}<250 \mathrm{MeV}$ cut but is not yet ready for inclusion in the analysis presented in this thesis. In the future it is hoped that this event selection will be incorporated into the $M_{A}^{Q E L}$ extraction because it selects higher $Q^{2}$ events than the sample obtained with just the hadronic energy cut. Figure 60 illustrates the higher $Q^{2}$ reach of the two-prong selection.

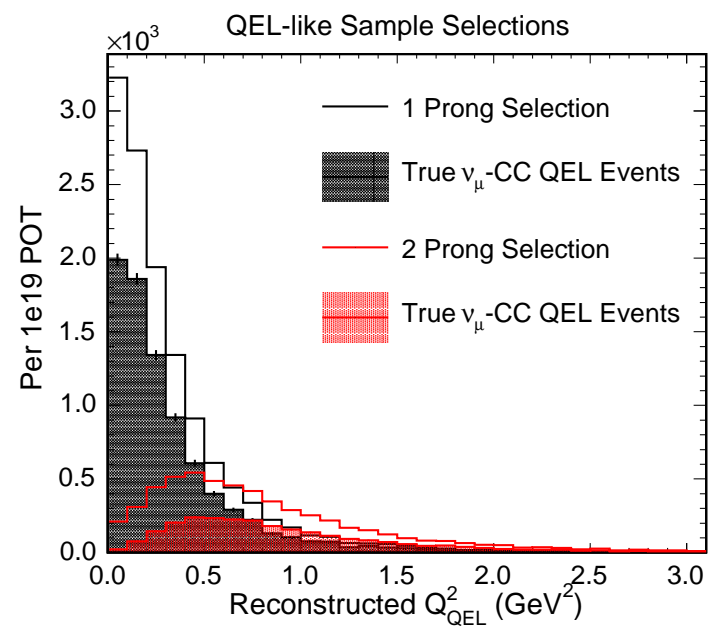

Figure 60: Selected QEL-like samples in MC as a function of the reconstructed $Q_{Q E L}^{2}$ using the oneprong (hadronic energy cut) and two-prong methods. The solid lines show the total selected samples whilst the shaded histograms show the true QEL event contributions to these samples. The two selection methods are complimentary with the one-prong method selecting primarily lower $Q_{Q E L}^{2}$ events and the two-prong method having a higher reach in $Q_{Q E L}^{2}$.

Figure 61 shows the efficiency and purity, relative to the CC-like sample, for selecting $\mathrm{v}_{\mu}$-CC QEL events as a function of the reconstructed $Q^{2}$ and $E_{\mathrm{v}}$. The purity is relatively flat as a function of $Q^{2}$ but the efficiency drops-off because the higher $Q^{2}$ events tend to have larger reconstructed hadronic energies. As a function of $E_{\mathrm{v}}$ the purity initially rises (and conversely the efficiency drops) reflecting the fact that at very low neutrino energies all interaction types are likely to leave only small amounts of visible hadronic energy whereas at 3-4 GeV the RES, 
DIS and NC events will produce larger showers in the detector and not pass the QEL-like selection cut. Above $4 \mathrm{GeV}$ the efficiency and purity flatten-off corresponding to the greater chance that even the true QEL events deposit more than $250 \mathrm{MeV}$ of hadronic energy in the detector.

Figure 61 also shows that these features are stable if the hadronic energy cut value is changed to either $200 \mathrm{MeV}$ or $300 \mathrm{MeV}$. Table 7 shows the numbers of events and percentages of the total QEL-like sample for the main interaction types. The major contamination comes from RES events where either the outgoing proton or pion is absorbed in the nucleus leading to little visible hadronic energy in the detector. Such events are kinematically very similar to the QEL signal events and, to the extent that they can be called background, this background source is irreducible when using the hadronic energy cut. The remainder of the QEL-like sample consists of $\sim 10 \%$ DIS events and then small contributions from NC and $\bar{v}_{\mu}$ interactions.
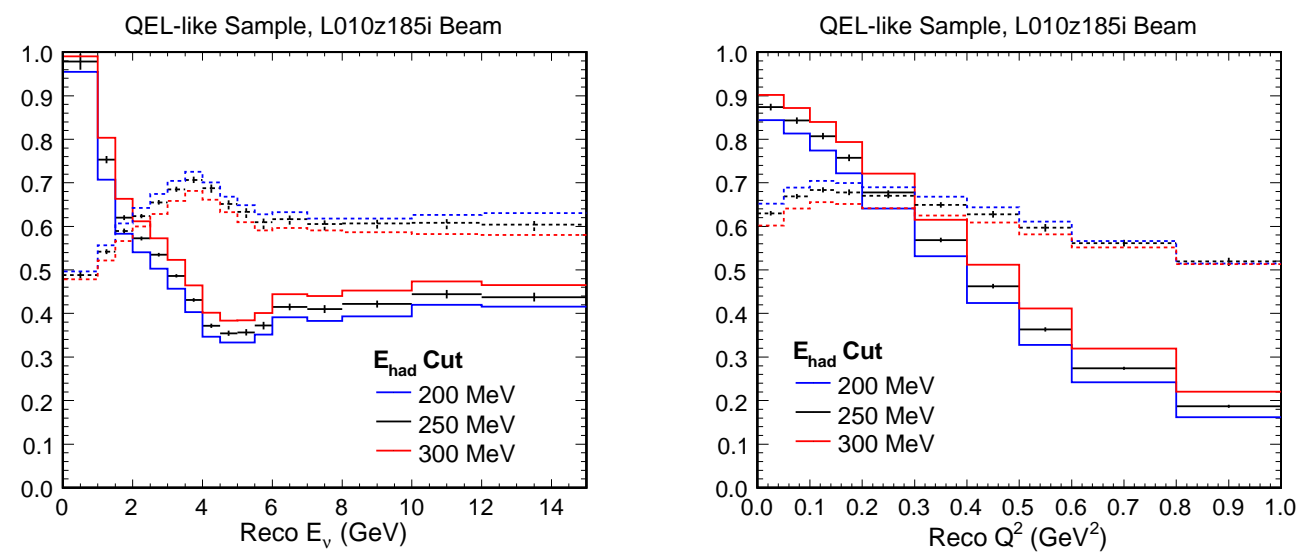

Figure 61: Efficiency (solid lines) and purity (dashed lines) of the selected QEL-like event sample, relative to the CC-like sample, as a function of the reconstructed neutrino energy and the reconstructed $Q^{2}$ for three different values of the reconstructed hadronic energy cut.

\subsubsection{Comparison of Data to Monte Carlo}

Figures 62 and 63 show the reconstructed QEL-assumed neutrino energy and squared fourmomentum transfer (as defined by equations 51 and 52) for QEL-like events and assume the same conventions about beam configuration, POTs and MC tuning that were described when 


\begin{tabular}{|c|c|c|}
\hline Interaction Type & $\begin{array}{c}\text { Events per } \\
\text { 1e20 POT }\end{array}$ & $\begin{array}{c}\text { Percentage } \\
\text { of Total }\end{array}$ \\
\hline \hline$v_{\mu}$-CC QEL & 168970.1 & 62.97 \\
\hline$v_{\mu}$-CC RES & 61064.0 & 22.76 \\
\hline$v_{\mu}$-CC DIS & 31402.4 & 11.70 \\
\hline$v_{\mu}$-CC COH & 1270.0 & 0.47 \\
\hline$v_{\mu}$-NC & 4023.5 & 1.50 \\
\hline$\overline{v_{\mu}}$ & 1307.0 & 0.49 \\
\hline
\end{tabular}

Table 7: Numbers of selected QEL-like events per 1e20 POT in nominal MC broken down by true interaction type.

presenting the data and $\mathrm{MC}$ comparisons for the $\mathrm{CC}$-like sample. The $\mathrm{MC}$ deficit that was evident in the data and MC comparisons for the CC-like sample is magnified with individual histogram bins having up to $40 \%$ more events in data. It can be seen that the tuned MC does agree slightly better with the data than the nominal MC although the beam and hadron production reweighting does not have a large effect on these predominantly lower neutrino energy events.

Figures 62 and 63 show that the QEL-like sample is not well modelled by the MC and that the $\mathrm{MC}$ reweighting process, that tries to improve the agreement between data and $\mathrm{MC}$ using parameters that change the incident neutrino flux, has little effect. The large data excess could be addressed in terms of an increase in the QEL cross section in the MC which would in turn correspond to an increase in the axial-vector mass, $M_{A}^{Q E L}$. 

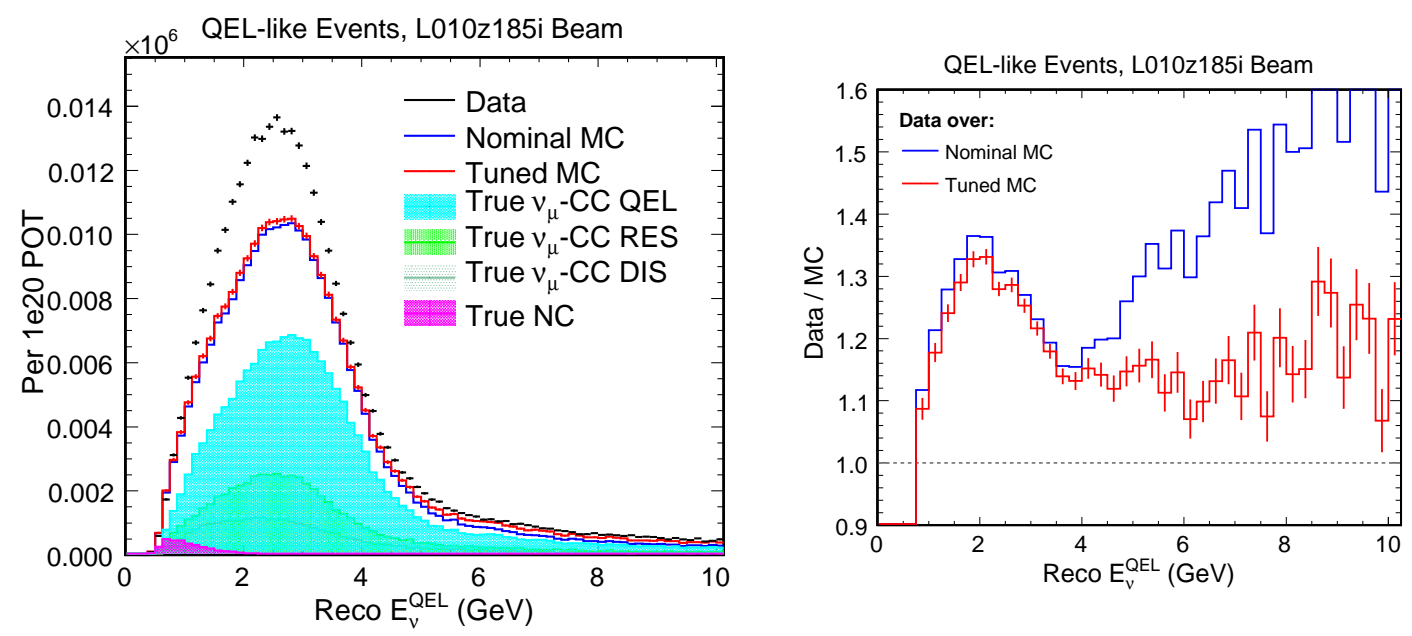

Figure 62: Reconstructed QEL-assumed neutrino energy for QEL-like events in data, nominal MC and tuned MC (left-hand figure) and ratios of data over MC (right-hand figure).
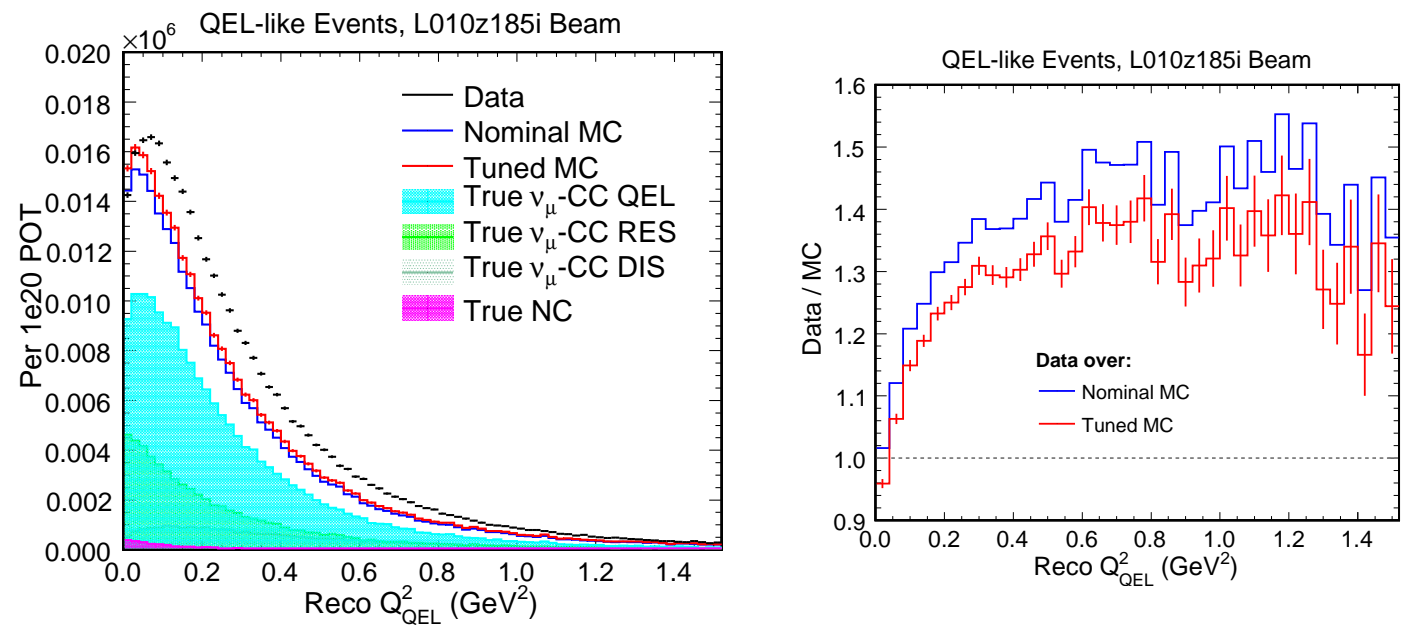

Figure 63: Reconstructed QEL-assumed squared four-momentum transfer for QEL-like events in data, nominal MC and tuned MC (left-hand figure) and ratios of data over MC (right-hand figure). 


\subsection{Near Detector Kinematic Resolutions}

In this section the MC will be used to quantify the ND resolutions for a numbers of kinematic variables that are of importance to the $M_{\AA}^{Q E L}$ extraction to be presented in the coming chapters. This information is used to determine the choice of distribution binnings to be used in the $M_{A}^{Q E L}$ fits but also allows the reader to further judge the quality of the data taken in the Near Detector beyond the data/MC comparisons shown previously.

\subsubsection{Resolution Fitting Method}

The resolution analysis proceeds by considering distributions of reconstructed minus truth $(R-$ $T$ ) variables in a number of slices of a certain truth variable. In each of these slices the $R-T$ distribution is fitted with a log-normal distribution which may be written as:

$$
f(x, \mu, \sigma, t, N)=N \exp \left\{-\frac{1}{2}\left[\log \left(1+t\left(\frac{x-\mu}{\sigma}\right) \frac{\sinh (t K)}{t K}\right)\right]^{2}+\frac{t^{2}}{2}\right\}
$$

where $K=\sqrt{\log (4)}, x$ is the variable in question, $\mu$ is the mean, $\sigma$ is the width, $t$ is a tail parameter and $N$ a normalisation factor. This functional form describes a Gaussian distribution with a one-sided tail. In the limit that $t \simeq 0$ the fitted function is given by:

$$
f(x, \mu, \sigma) \propto N \exp \left[-\frac{1}{2}\left(\frac{x-\mu}{\sigma}\right)^{2}\right]
$$

Figure 64 shows an example of the fitted $R-T Q^{2}$ distributions for true $v_{\mu}$-CC events in the CC-like sample in a number of slices of true $Q^{2}$. In the resolution fitting analysis only the true $v_{\mu}$-CC (or true $v_{\mu}$-CC QEL) events that are selected as CC-like are considered in order to remove contaminations, such as $\mathrm{NC}$ events, that will bias the extracted resolutions.

The log-normal form provides a better estimate of the mean and width than can be obtained either directly from the distributions (or via a standard Gaussian fit) by neglecting the contributions of the tails of the $R-T$ distributions. The Gaussian means from the log-normal fits correspond to the pull on the resolution and provide an estimate for the accuracy of the reconstruction. The Gaussian widths provide an estimate of the resolution (precision of the 

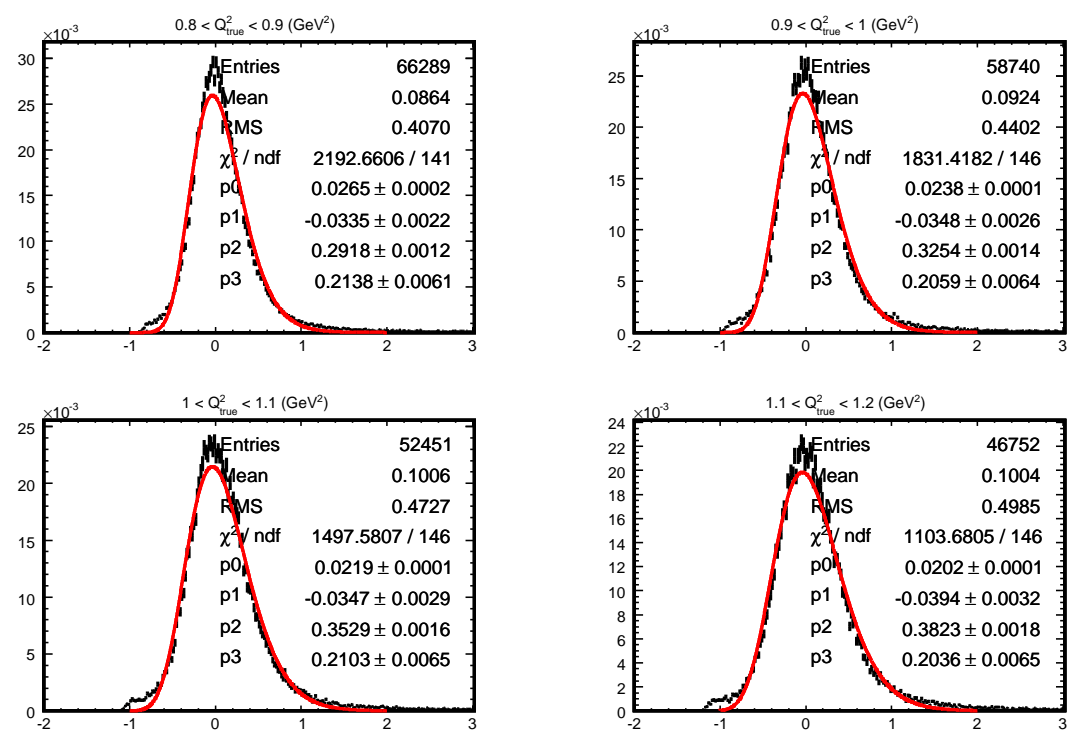

\section{$\mathrm{Q}_{\text {reco }}^{2}-\mathrm{Q}_{\text {true }}^{2}$ : True $v_{\mu}-\mathrm{CC}$ Events in CC-like Sample}

Figure 64: Distributions of $R-T Q^{2}$ for true $v_{\mu}$-CC events in the CC-like sample in four slices of true $Q^{2}$. The red lines show the fitted log-normal distributions and the parameter 00 corresponds to the overall normalisation, $p 1$ to the Gaussian mean, $p 2$ to the Gaussian width and $p 3$ to the tail parameter.

reconstruction) for the variable in question. The Gaussian widths fitted in each slice can then be plotted as a function of the truth variable in question and this distribution fitted to provide a functional form to describe the detector resolution.

\subsubsection{Results for ND Kinematic Resolutions}

Using the methodology described above, this section will present the ND resolutions for the muon energy, the muon angle (with respect to the incident neutrino direction) and the resulting squared four-momentum transfer for true $v_{\mu}$-CC and true $v_{\mu}$-CC QEL events that are selected as being CC-like. The resolution analysis uses the same MC sample and selection criteria as were described previously in this chapter.

The muon energy resolution is broken down into two classes; the resolution for muons that stop in the detector and whose momentum is measured using range and the resolution for muons that exit the detector and whose momentum is measured by curvature in the magnetic field. In the $M_{A}^{Q E L}$ extraction from ND data, to be presented in the coming chapters, only events with muons 
that stop in the detector are considered and so this section will just present the resolution for the muon energy as measured by range. Figure 65 shows the fitted Gaussian means and widths (from the log-normal fits to the $R-T$ muon energy distributions in slices of true muon energy) as a function of true muon energy for true $v_{\mu}$-CC events in the CC-like sample and for true $v_{\mu}$-CC QEL events in the CC-like sample.

Figure 65 shows that above $1 \mathrm{GeV}$ the fractional resolution for the reconstruction of the muon energy, as measured by range in the detector, is almost flat as a function of the true muon energy for both true CC and true QEL events. The fractional resolution is $\sim 5-8 \%$ with fractional biases in the reconstructed value of less than $2 \%$ below $8 \mathrm{GeV}$. Above $8 \mathrm{GeV}$ there are almost no events containing stopping muons and the drop-off to larger negative biases that can be observed in the fractional pull distributions from figure 65 is likely due to muon tracks being incorrectly assigned as stopping in the detector leading to an under-estimate of their energy. Below $1 \mathrm{GeV}$ the muon energy resolution increases and this is because very low energy tracks contain few hits and are more susceptible to mis-assignment of hadronic shower hits to the track or track hits to the hadronic shower. It can be seen that this effect is reduced for the true QEL events (and also that the resolution for low energy muons in general is improved for the QEL events) because of the small size of the hadronic shower compared to the RES and DIS events. It should also be noted that for true QEL events the energy of the hadronic shower is small and so the resolution for the neutrino energy for true QEL events is essentially the same as the resolution for the muon energy as given by figure 65 .

Figure 66 shows the resolution and pull on the resolution for the muon scattering angle with respect to the incident neutrino direction, $\theta_{\mu}$, for true $v_{\mu}$-CC and true $v_{\mu}$-CC QEL events, that are selected as being CC-like, as a function of the true scattering angle. In both cases the absolute resolution for the angle increases almost linearly as a function of the true angle as might be expected given that lower angles correspond to higher energy muons, which leave longer and straighter tracks in the detector, for which the angle is better constrained. The fractional resolution for the angle is better for the true QEL events than for the true CC events because the smaller hadronic showers reduce the possibilty of mis-reconstruction of the track vertex and the first hits on the muon track. Figure 66 also shows that the fractional pull on the 

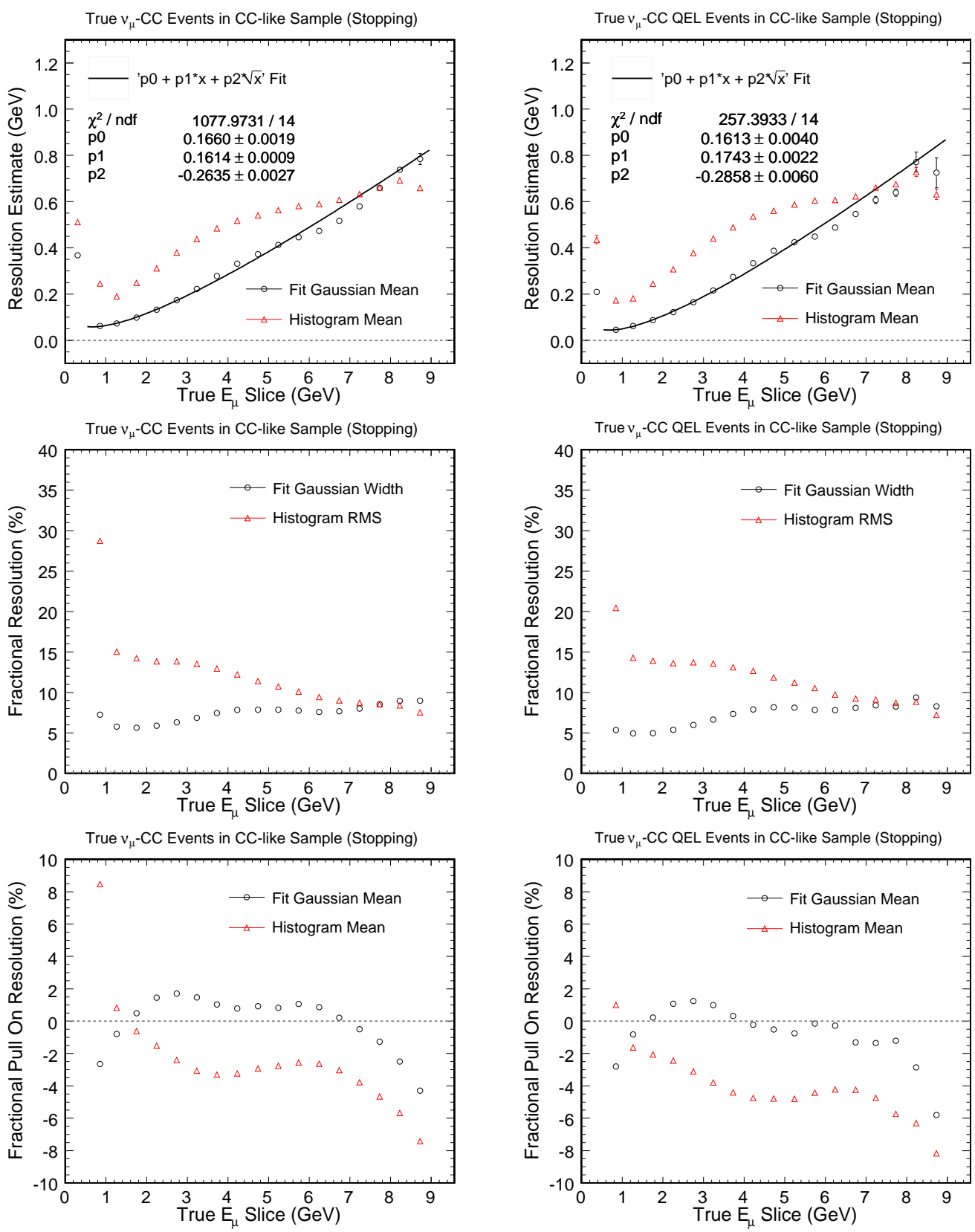

Figure 65: The left-hand figures correspond to true $\mathrm{v}_{\mu}$-CC events in the CC-like sample whilst the righthand figures correspond to true $v_{\mu}$-CC QEL events in the CC-like sample. The top figures show the Gaussian widths from the log-normal fits to the $R-T$ distributions as black circles along with the $R M S$ values of the $R-T$ histograms as red triangles. The black curves correspond to a fitted parameterisation of the detector resolution and all points are plotted at the mean value of the true muon energy in a given slice rather than at the centre of that slice to make the parameterisations more accurate. The second row of figures show the fractional fitted Gaussian widths and $R-T$ histogram RMS values whilst the bottom row of figures show the fractional fitted Gaussian means and $R-T$ histogram means. In all cases the differences between the black circles and red triangles correspond to the influence of the tails of the $R-T$ distributions. 

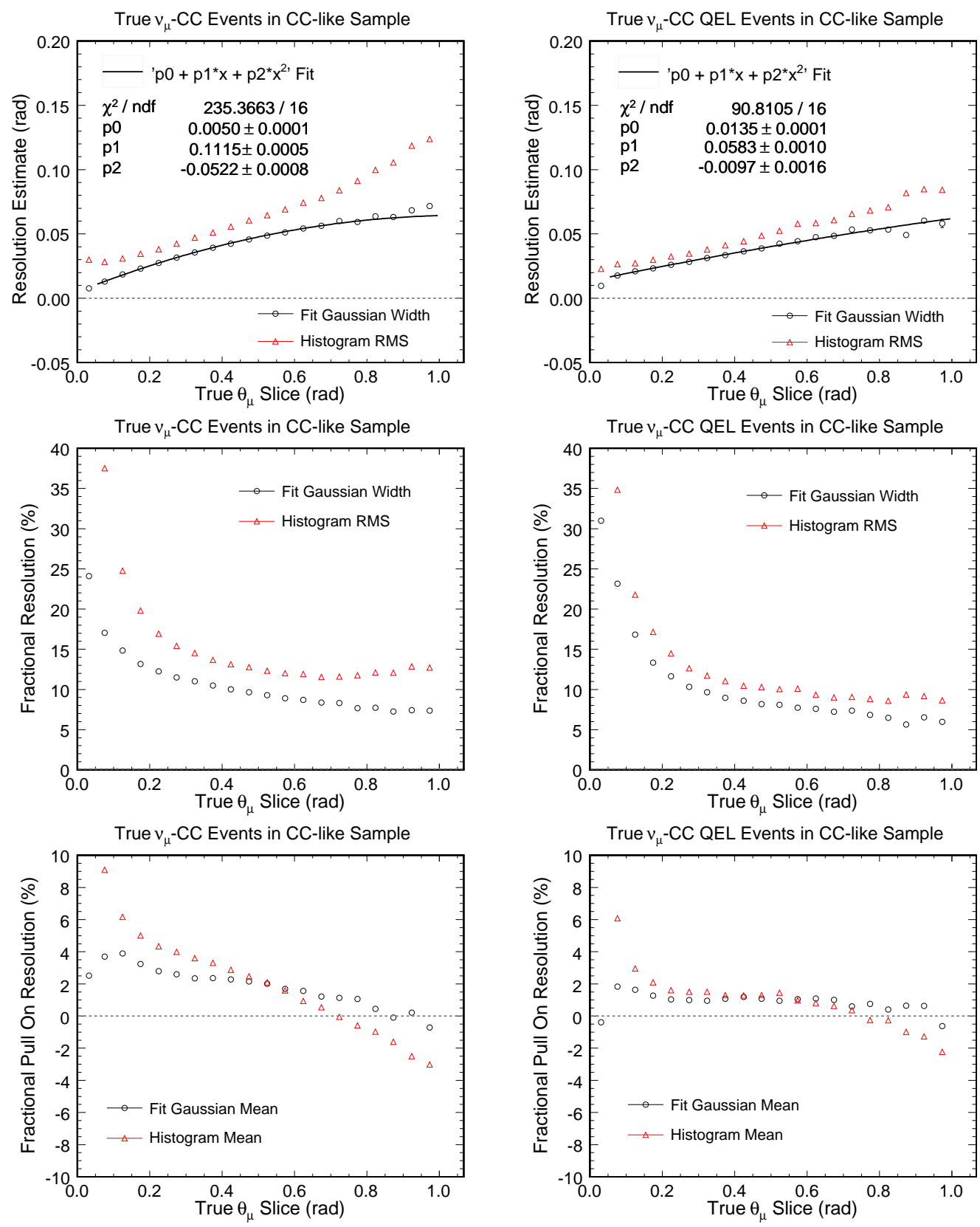

Figure 66: The left-hand figures correspond to true $v_{\mu}$-CC events in the CC-like sample whilst the righthand figures correspond to true $\mathrm{v}_{\mu}$-CC QEL events in the CC-like sample. The top figures show the Gaussian widths from the log-normal fits to the $R-T$ distributions as black circles along with the $R M S$ values of the $R-T$ histograms as red triangles. The black curves correspond to a fitted parameterisation of the detector resolution and all points are plotted at the mean value of the true muon scattering angle in a given slice rather than at the centre of that slice to make the parameterisations more accurate. The second row of figures show the fractional fitted Gaussian widths and $R-T$ histogram RMS values whilst the bottom row of figures show the fractional fitted Gaussian means and $R-T$ histogram means. In all cases the differences between the black circles and red triangles correspond to the influence of the tails of the $R-T$ distributions. 
resolution is at most $4 \%$ for the true CC events and at most $2 \%$ for the true QEL events and that these fractional pulls are everywhere smaller than the fractional resolutions for the scattering angle.

The squared four-momentum transfer is reconstructed using the above variables and figure 67 shows the resolution for $Q^{2}$ for the true $v_{\mu}$-CC events in the CC-like sample and for $Q_{Q E L}^{2}$ for the true $v_{\mu}$-CC QEL events in the CC-like sample with both sets of figures only considering events where the muon stopped in the detector. In both cases the resolution rises almost linearly with the true $Q^{2}$ whilst the fractional resolution improves as the true $Q^{2}$ increases. The $Q^{2} / Q_{Q E L}^{2}$ fractional resolution is a convolution of the fractional resolution for the muon energy and the fractional resolution for the muon scattering angle and it can be seen that it takes the expected shape given the dependance of $Q^{2}$ on the muon energy and angle (the most probable value for $Q^{2}$ is reasonably flat as a function of the muon energy whereas lower $Q^{2}$ events tend to correspond to muons with lower scattering angles). The resolution for $Q^{2}$ for the true QEL events is better than that for the true CC events and this effect can again be attributed to the 'cleaner' hadronic environment. The modulus of the fractional bias on the reconstructed $Q^{2}$ is at most $\sim 15 \%$ for both sets of events and is everywhere smaller than the fractional resolution. 

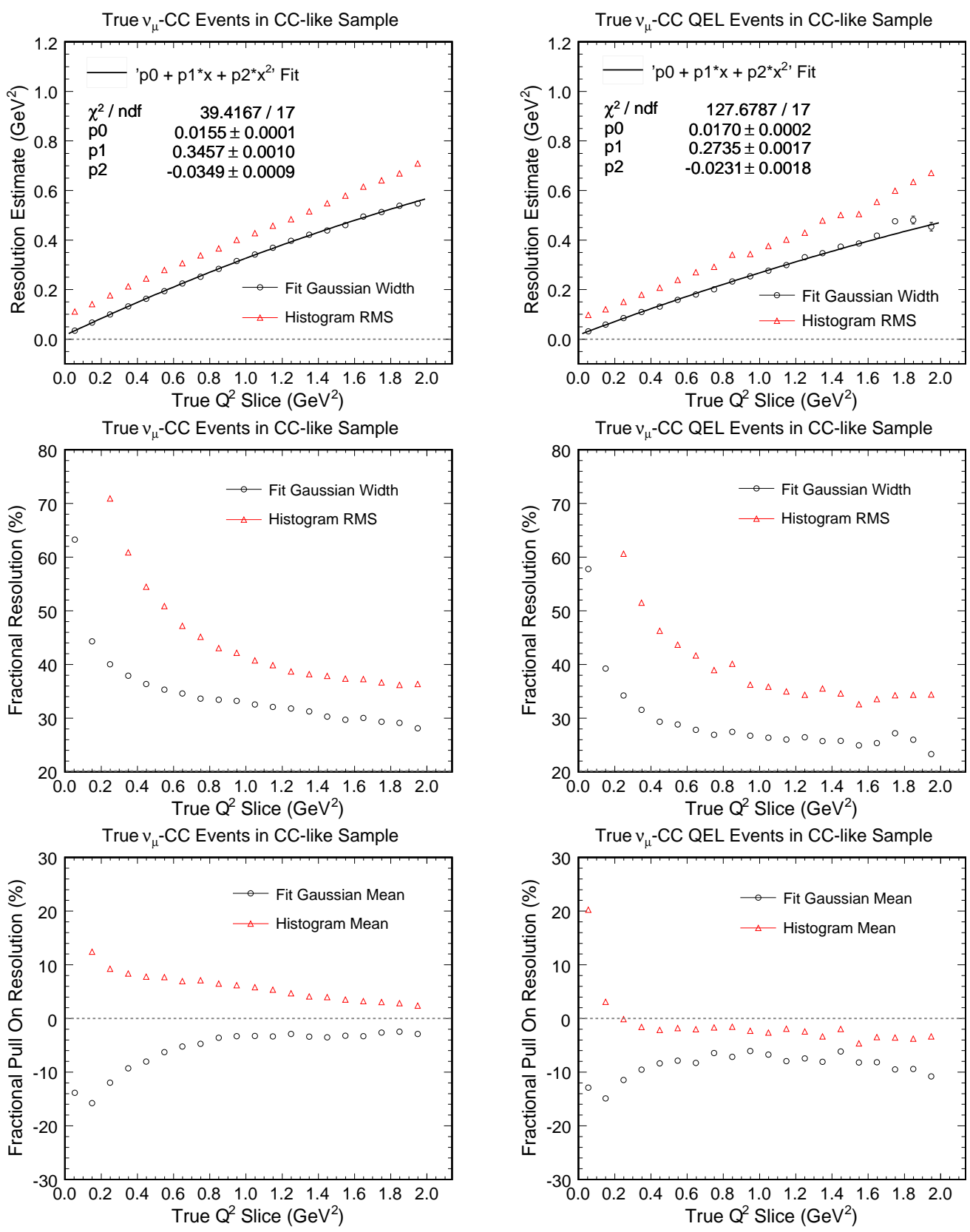

Figure 67: The left-hand figures correspond to true $v_{\mu}$-CC events in the CC-like sample whilst the righthand figures correspond to true $v_{\mu}$-CC QEL events in the CC-like sample and both sets of figures only use events containing muons that stopped in the detector. The top figures show the Gaussian widths from the log-normal fits to the $R-T$ distributions as black circles along with the RMS values of the $R-T$ histograms as red triangles. The black curves correspond to a fitted parameterisation of the detector resolution and all points are plotted at the mean value of the true squared four-momentum transfer in a given slice rather than at the centre of that slice to make the parameterisations more accurate. The second row of figures show the fractional fitted Gaussian widths and $R-T$ histogram RMS values whilst the bottom row of figures show the fractional fitted Gaussian means and $R-T$ histogram means. In all cases the differences between the black circles and red triangles correspond to the influence of the tails of the $R-T$ distributions. 


\section{$7 M_{A}^{Q E L}$ Fit Procedure}

This chapter will introduce the fit method that will be used to extract $M_{A}^{Q E L}$ from MINOS ND data. The first section will present an overview of the fit with the following two sections discussing the fit parameters and configurations in more detail. Next some fit convergence issues will be highlighted and a method, based on the smearing of $\mathrm{MC}$ event variables, to alleviate these problems will be introduced. The subsequent section will show an example fit to a mock data sample before the final section presents a number of studies aimed at establishing the robustness and accuracy of the fitting method.

\subsection{Overview of the Fit}

The MC QEL-like sample reconstructed $Q_{Q E L}^{2}$ and $E_{\mathrm{v}}^{Q E L}$ distributions (where these variables are defined by equations 52 and 51) are fit to the data in an iterative procedure in which the underlying parameters, in particular $M_{A}^{Q E L}$ (via a scale factor applied to it's nominal NEUGEN value of $0.99 \mathrm{GeV}$ ), are varied and event weights re-calculated. This procedure uses the MC directly to account for acceptance effects.

Two different fitting techniques have been developed. The first fits the data using only the $Q_{Q E L}^{2}$ distribution, in a method similar to that used by previous experiments such as those mentioned in chapter 3, and the second fits the data using both the $Q_{Q E L}^{2}$ and $E_{\mathrm{v}}^{Q E L}$ distributions, a method which allows for a more natural inclusion of neutrino flux information. The fit can be configured to use any combination of shape and/or normalisation information for these distributions and further details on the fit configuration will be presented in a later section. Figure 68 shows the effect of up to $\pm 20 \%$ changes to the value of $M_{A}^{Q E L}$ on the QEL-like sample $Q_{Q E L}^{2}$ and $E_{\mathrm{v}}^{Q E L}$ distributions and illustrates the shape and normalisation features that the fit will use to constrain $M_{A}^{Q E L}$.

The fit is performed using the MINUIT package to minimise one of the following functions:

$$
-2 \mathscr{L}=\sum_{i=1}^{n \text { Bins }} 2\left[e_{i}\left(\alpha_{1}, \ldots, \alpha_{N}\right)-o_{i}+o_{i} \log \left(\frac{o_{i}}{e_{i}\left(\alpha_{1}, \ldots, \alpha_{N}\right)}\right)\right]+\sum_{j=2}^{N} \frac{\Delta \alpha_{j}^{2}}{\sigma_{\alpha_{j}}^{2}}
$$



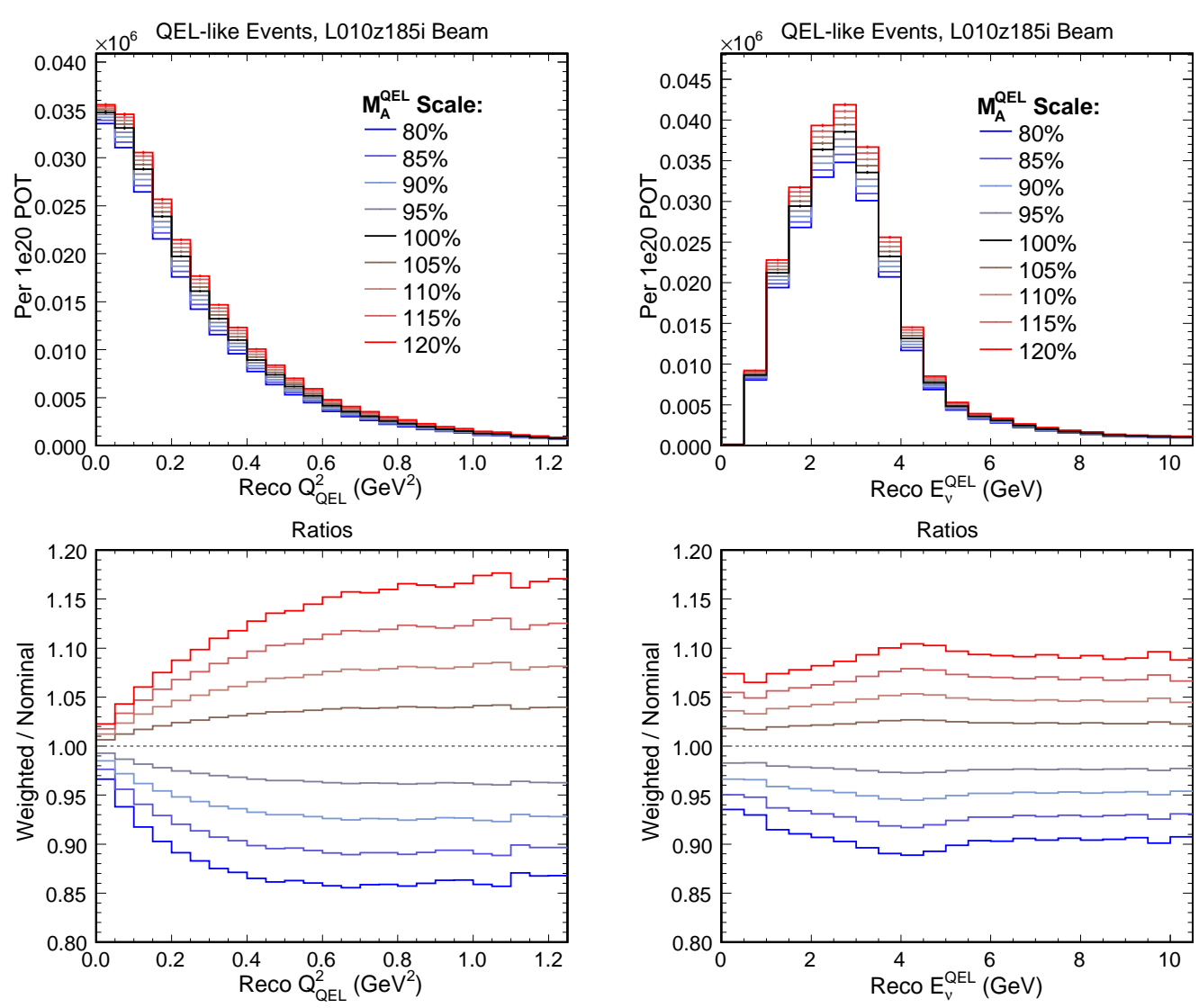

Figure 68: Effect of up to $\pm 20 \%$ changes to the value of $M_{A}^{Q E L}$ upon the $Q_{Q E L}^{2}$ and $E_{\mathrm{v}}^{Q E L}$ distributions of $Q E L$-like events in $M C$. The left-hand plots show $Q_{Q E L}^{2}$ and illustrate that there is both a change in shape and normalisation when $M_{A}^{Q E L}$ is varied. The positive and negative changes are not symmetric due to the presence of non- $v_{\mu}$-CC QEL events in the QEL-like sample; such events are not reweighted when $M_{A}^{Q E L}$ is varied. The right-hand plots show $E_{v}^{Q E L}$ and as expected, given that the QEL cross section is flat down to neutrino energies of $\sim 1 \mathrm{GeV}$ (see figure 13), the effect of changing the value of $M_{A}^{Q E L}$ is largely a change in the normalisation of the distribution.

$$
\begin{gathered}
\chi^{2}=\sum_{i=1}^{n B i n s} \frac{\left(o_{i}-e_{i}\left(\alpha_{1}, \ldots, \alpha_{N}\right)\right)^{2}}{o_{i}}+\sum_{j=2}^{N} \frac{\Delta \alpha_{j}^{2}}{\sigma_{\alpha_{j}}^{2}} \\
\chi_{M \text { Cstats }}^{2}=\sum_{i=1}^{n \text { Bins }} \frac{\left(o_{i}-e_{i}\left(\alpha_{1}, \ldots, \alpha_{N}\right)\right)^{2}}{o_{i}+S e_{i}\left(\alpha_{1}, \ldots, \alpha_{N}\right)}+\sum_{j=2}^{N} \frac{\Delta \alpha_{j}^{2}}{\sigma_{\alpha_{j}}^{2}}
\end{gathered}
$$


where $o_{i}$ is the observed number of events in data for bin $i, e_{i}\left(\alpha_{1}, \ldots, \alpha_{N}\right)$ is the expected number of events in MC for bin $i$ given the fit parameters, $\alpha_{j}$ is the $j^{\text {th }}$ fit parameter, $S$ is the scale factor applied to normalize the MC histogram and the penalty term is computed with $\Delta \alpha_{j}$, the shift from nominal for the $j^{\text {th }}$ systematic parameter, and $\sigma_{\alpha_{j}}$, the assumed $1 \sigma$ error on the $j^{t h}$ systematic parameter. The first fit parameter $\alpha_{1} \equiv M_{A}^{Q E L}$ and this is treated as a free parameter in the fit and does not influence the penalty term. Note that the sum over bins could refer to just the bins of the $Q_{Q E L}^{2}$ distribution or both the bins of the $Q_{Q E L}^{2}$ and $E_{\mathrm{V}}^{Q E L}$ distributions depending upon the fit configuration.

The $-2 \mathscr{L}$ function is appropriate for cases where the sample has few events because it assumes an underlying Poisson nature for the bin contents and was used when only small samples of data were available. The $\chi^{2}$ and $\chi_{M C s t a t s}^{2}$ functions both assume a Gaussian nature for the bin contents and in the limit of infinite statistics $\chi^{2}$ should give the same result as $-2 \mathscr{L}$ (in practice if there are more than $\sim 100$ events per bin the two functions will give almost identical results). The $\chi_{M C s t a t s}^{2}$ function is an attempt to include the statistical uncertainties in the MC directly although this is not a trivial addition and in the future a more sophisticated treatment, such as that presented in [79], could be adopted.

\subsection{Fit Parameters}

In addition to considering $M_{A}^{Q E L}$ as a free parameter the fit also includes a number of systematic parameters that contribute both to the portion of $\chi^{2}$ coming from bin contents of the fitted distribution(s) and also to the penalty term. The following four sources are considered to be the dominant systematic uncertainties on the extraction of $M_{A}^{Q E L}$ from MINOS ND data and are directly included in the fit:

- Muon energy scale: the muon energy enters directly into the computation of $Q_{Q E L}^{2}$ for a given event and changes to this parameter will move events to lower or higher $Q_{Q E L}^{2}$ values. For the QEL-like events the muon energy dominates the reconstruction of the neutrino energy and so changes to this parameter will also move events to lower or higher $E_{\mathrm{v}}^{Q E L}$ values. An assumed $1 \sigma$ error of $2 \%$ is used in the penalty term for the muon 
energy scale parameter as justified in [11]. Figure 69 shows the effect of $\pm 2 \%$ changes to the muon energy scale.


Figure 69: Effect of $\pm 2 \%$ changes to the muon energy scale on the $Q_{Q E L}^{2}$ (left-hand plots) and $E_{\mathrm{v}}^{Q E L}$ (right-hand plots) distributions for QEL-like events in MC. The muon energy scale parameter does not change the weight given to a particular MC event but rather distorts the shapes of the two distributions.

Recently the ND magnetic field has been re-calibrated and results showed that it increased by $12.3 \%$ (averaged over the detector). This change shifts the muon momentum scale for muons that exit the detector (with momentum measured by curvature) by $\sim 6 \%$ [11] but has a minimal effect upon the momentum measurement for muons that stop in the detector for which the path-length corrected range and detector material stoppingpower are used to reconstruct the momentum. As a result only muons that stop in the ND are used when fitting the data for $M_{A}^{Q E L}$ (the previously shown data and MC comparisons and the mock data fit studies use both stopping and exiting muons). This does not result in a large loss of statistics as most of the QEL-like events correspond to lower energy muons that stop in the detector. 
The assumed error for the muon energy scale could be reduced in the future because the figure of $2 \%$ was calculated using all $\mathrm{v}_{\mu}$-CC interactions whereas this analysis only considers stopping muon events with small amounts of hadronic activity and for these events the uncertainty in the energy scale could be smaller. In particular, recent work [80] has shown that for low energy muons interacting in CalDet the data and MC agree to better than $2 \%$. Figure 70 shows that for $2 \mathrm{GeV}$ muons interacting in CalDet the positions of the peaks of the track length distributions, which are used to reconstruct the energy of these stopping muons, in data and MC agree to $1 \%$.

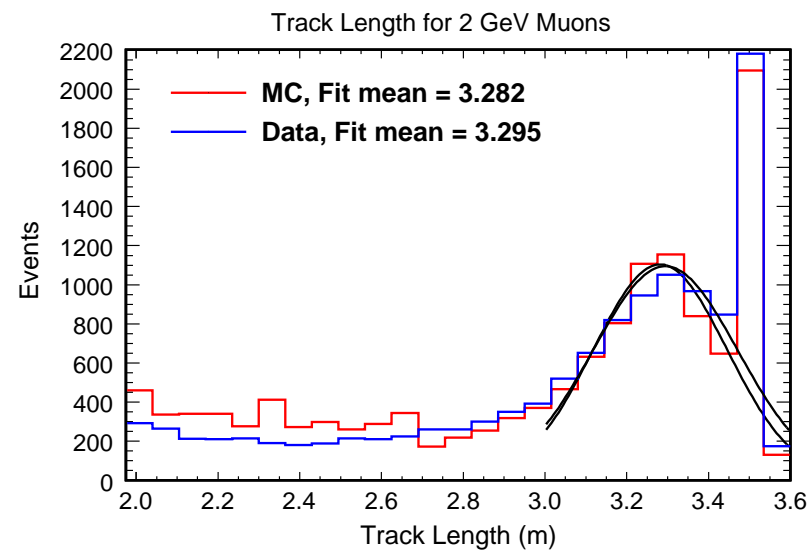

Figure 70: Comparisons of the track length of $2 \mathrm{GeV}$ muons for data and MC events in CalDet. Fits to the peaks of these distributions show that the means agree to 1\%. Figure taken from [80].

- Hadronic energy offset: the hadronic energy does not enter the reconstruction of $Q_{Q E L}^{2}$ or $E_{\mathrm{v}}^{Q E L}$ directly but changes these distributions by causing a different number of events to pass the hadronic energy cut that defines the QEL-like sample (as described in chapter 6). Figure 71 shows the average $Q^{2}$ and $E_{v}$ values for CC-like events as a function of the reconstructed shower energy. In the region of the QEL-like sample hadronic energy cut, at $250 \mathrm{MeV}$, there is a slope to these profiles and so changes to the hadronic energy offset will not just be reflected as a flat change in normalisation for the fitted $Q_{Q E L}^{2}$ and $E_{\mathrm{v}}^{Q E L}$ distributions but also will alter their shapes by a small amount.

This systematic parameter does encompass uncertainties in the detector modelling, cal- 

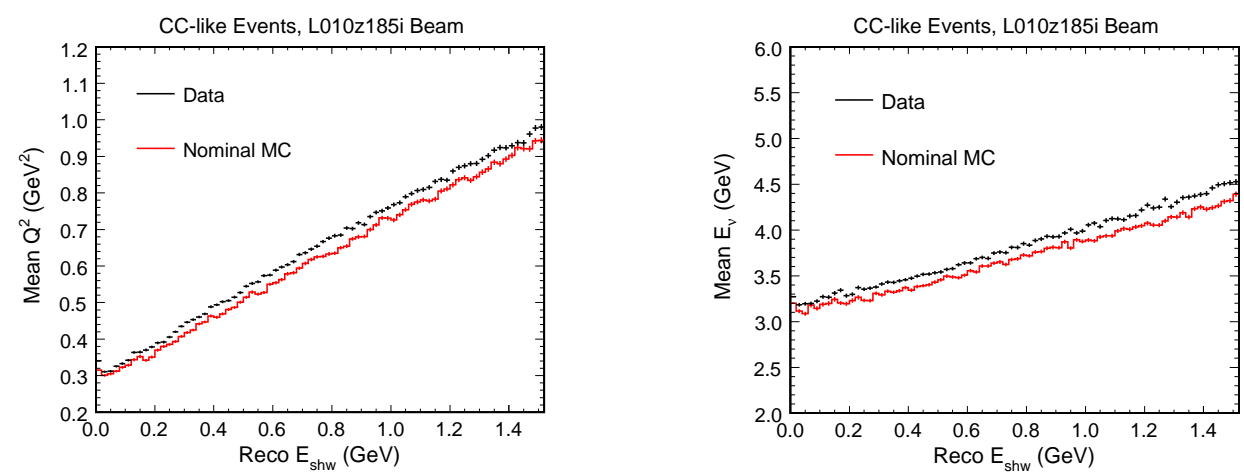

Figure 71: Mean $Q^{2}$ (left-hand plot) and $E_{\mathrm{v}}$ (right-hand plot) values in slices of reconstructed shower energy for CC-like events in data and nominal MC. Around the region of the QEL-like sample hadronic energy cut at $250 \mathrm{MeV}$ the distributions all exhibit slopes indicating that changes to the hadronic energy will induce small shape changes in the fitted $Q_{Q E L}^{2}$ and $E_{\mathrm{v}}^{Q E L}$ distributions.

ibration and reconstruction of hadronic showers but was chosen to be an offset rather than a scale factor that might be usually associated with such sources of uncertainty. The choice of an offset versus a scale is somewhat arbitrary but the offset was chosen because the dominant uncertainty for these low hadronic energy events comes from the potential mis-modelling of FSIs such as the probability that a proton in a QEL event, or a pion from a RES event, makes it out of the nucleus to deposit energy in the detector. Changes to the INTRANUKE model result in discrete changes in the reconstructed hadronic energy on an event-by-event basis (rather than a simple scaling law that depends upon the energy of the hadronic system) and studies of the effect of variations in INTRANUKE parameters support an assumed $1 \sigma$ error for the hadronic energy offset parameter of 35 $\mathrm{MeV}$. Figure 72 shows the effect of $\pm 35 \mathrm{MeV}$ changes to the hadronic energy.

- Inelastic background scale: the inelastic background scale parameter is applied to all non- $v_{\mu}$-CC QEL events in the QEL-like sample, the major component of which comes from $v_{\mu}$-CC RES events (which are kinematically similar to the signal). There is also a small but non-negligable contribution from DIS events. Previous MINOS analyses have used an uncertainty of $10 \%$ for resonant axial-vector mass [11] $\left(M_{A}^{1 \pi}\right.$ relates to the cross section for resonance production in a similar way to the relation between $M_{A}^{Q E L}$ and the cross section for QEL scattering) and a 3\% uncertainty for the normalisation of 

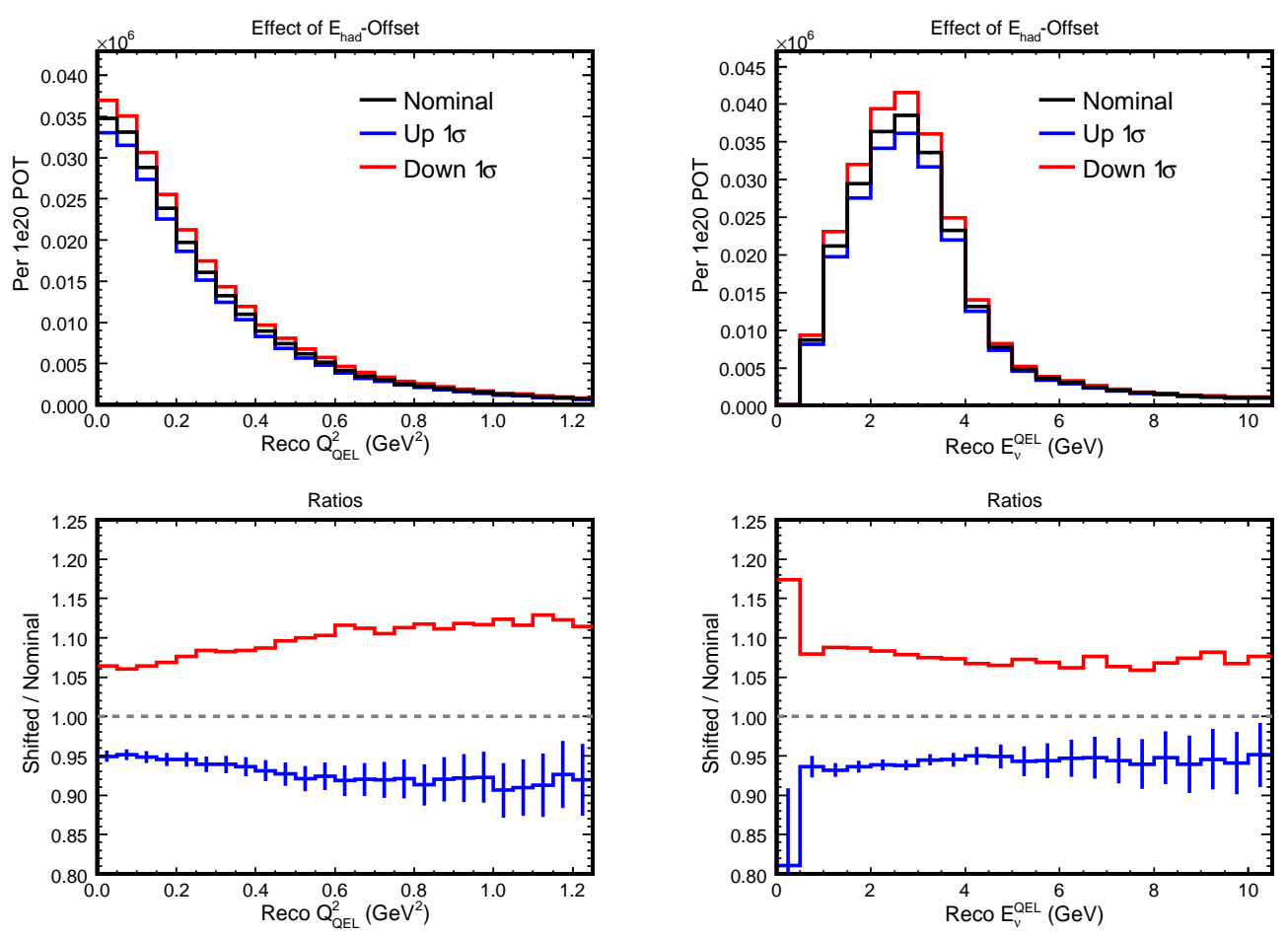

Figure 72: Effect of $\pm 35 \mathrm{MeV}$ changes to the hadronic energy offset on the $Q_{Q E L}^{2}$ (left-hand plots) and $E_{\mathrm{v}}^{Q E L}$ (right-hand plots) distributions for QEL-like events in MC. These changes cause more or less CC-like events to be classified as QEL-like and so predominantly effect the normalisation of the distributions.

the deep-inelastic scattering cross section at high invariant masses $(W>1.7 \mathrm{GeV})$. The DIS component of the QEL-like sample is small and located at lower invariant masses and so an assumed $1 \sigma$ uncertainty of $10 \%$ is assigned to the background scale parameter. Figure 73 shows the effect of $\pm 10 \%$ changes to the background scale.

This systematic parameter is fairly basic in that no attempts have yet been made to preserve the inclusive $v_{\mu}$-CC cross section at high energies (where it is well constrained experimentally) and also because it incorporates all the background interaction modes into a single parameter. In the future the background scale could be replaced by a number of systematic parameters that effect both the shape and normalisation of the background contributions to the QEL-like sample. Such parameters could include $M_{A}^{1 \pi}$, to alter the cross section for resonant inelastic processes, and changes to the parameters governing 



Figure 73: Effect of $\pm 10 \%$ changes to the background scale on the $Q_{Q E L}^{2}$ (left-hand plots) and $E_{\mathrm{v}}^{Q E L}$ (right-hand plots) distributions for QEL-like events in MC. These changes weight the non- $\mathrm{v}_{\mu}-C C Q E L$ events, which are distributed similarly to the signal in $Q_{Q E L}^{2}$ and $E_{\mathrm{v}}^{Q E L}$, and so mostly induce changes to the normalisation of the distributions. Note that this parameter does not change the shapes of the background distributions in $Q_{Q E L}^{2}$ and $E_{\mathrm{v}}^{Q E L}$.

the KNO scaling model (as mentioned in chapter 5), to alter the non-resonant inelastic scattering events.

- Incident neutrino flux: the last systematic uncertainty that can be included in the fit corresponds to uncertainties in both the shape and normalisation of the incident neutrino flux. This uncertainty is accomodated by a number of 'flux factors' which act as scale factors for slices of the true neutrino energy distribution. Currently seven slices are considered, defined as 0-2, 2-3, 3-4, 4-6, 6-10, 10-20 and 20-40 GeV with each flux factor acting as a weight for events whose true neutrino energies lie in that particular slice. Studies of the beam and hadron production parameter reweighting procedure show that a reasonable estimate for the $1 \sigma$ errors on the flux factors is $8 \%$ for $E_{v}^{\text {true }} \leq 6 \mathrm{GeV}$ 
and $15 \%$ for $E_{v}^{\text {true }}>6 \mathrm{GeV}$. Figure 74 shows the effect of $\pm 8 \%$ changes to the true neutrino flux between 3 and $4 \mathrm{GeV}$.
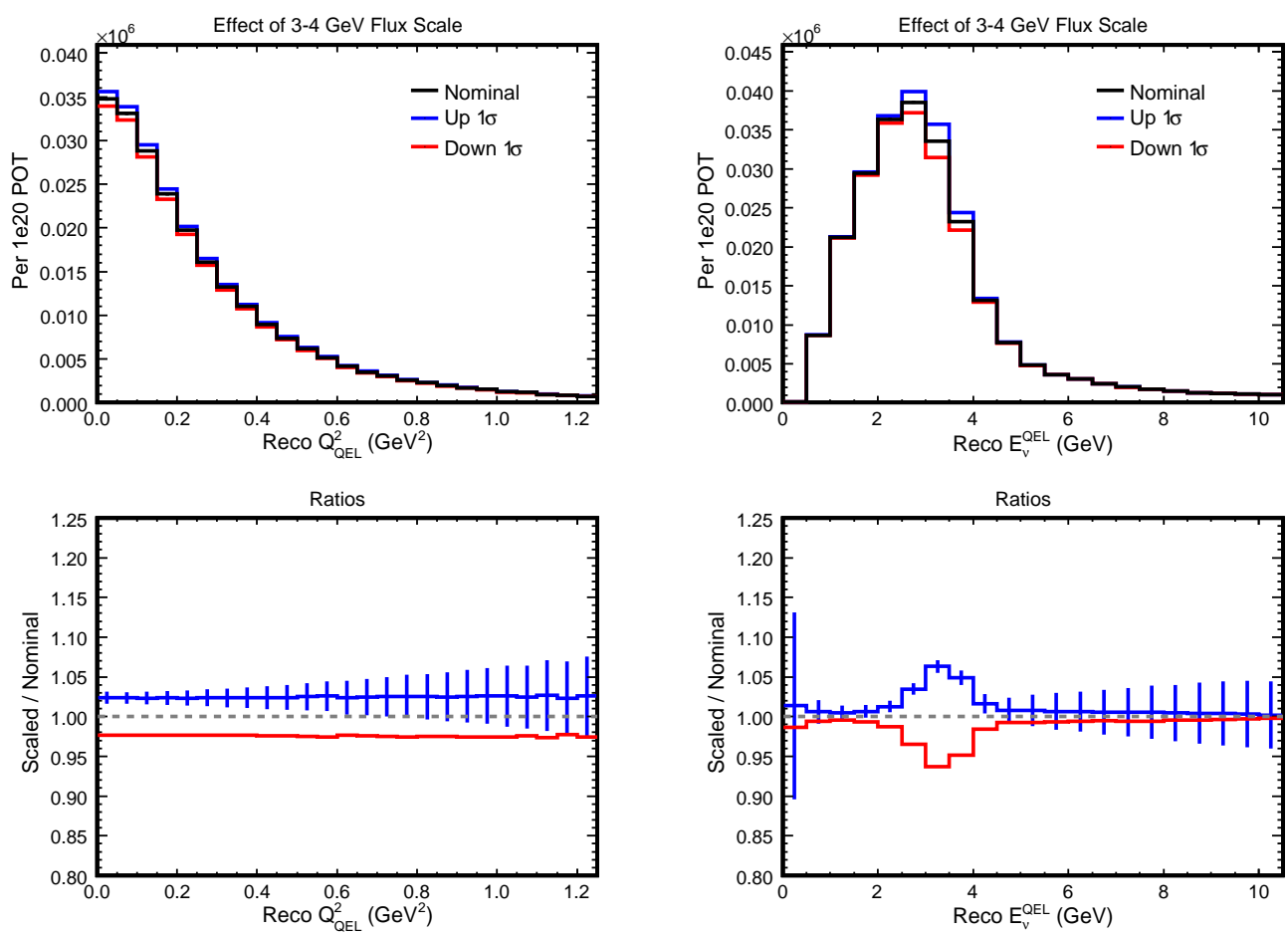

Figure 74: Effect of $\pm 8 \%$ changes to the neutrino flux for events with $E_{v}^{\text {true }} \in[3.0,4.0) \mathrm{GeV}$ on the $Q_{Q E L}^{2}$ (left-hand plots) and $E_{\mathrm{v}}^{Q E L}$ (right-hand plots) distributions for QEL-like events in MC. Changes to the neutrino flux have little effect on the shape of the $Q_{Q E L}^{2}$ distribution except at the lowest neutrino energies. Changes to the flux have a large effect on the $E_{\mathrm{v}}^{Q E L}$ distribution and, because the flux factors are based upon the true neutrino energy, this scaling is smeared over a number of bins.

These flux factors simply weight large 'chunks' of the neutrino energy spectrum and in the future a more sophisticated method could be introduced to smooth the weights that get applied to events from adjacent flux factor slices. A further advancement that could be considered would be to combine the beam and hadron production fitting procedure (as described in chapter 6) with the $M_{A}^{Q E L}$ fitting procedure.

One problem with the use of the flux factors is that the slice-to-slice variations in the scale values should not be allowed to differ by large amounts as this would introduce 
unreasonable discontinuities and warping into the true neutrino energy spectrum. The fit has been designed to calculate a penalty term for the flux factors that differs from that presented in equations $85-87$ and uses a full covariance matrix equation according to:

$$
\Delta \alpha^{\mathbf{T}} \mathbf{V}^{-1} \Delta \alpha=\left(\Delta \alpha_{k}, \ldots, \Delta \alpha_{N}\right)\left(\begin{array}{cc}
\rho_{\alpha_{k} \alpha_{k}} \sigma_{\alpha_{k}}{ }^{2} & \cdots \\
\rho_{\alpha_{k+1} \alpha_{k}} \sigma_{\alpha_{k+1}} \sigma_{\alpha_{k}} & \ddots \\
\vdots &
\end{array}\right)^{-1}\left(\begin{array}{c}
\Delta_{\alpha_{k}} \\
\vdots \\
\Delta_{\alpha_{N}}
\end{array}\right)
$$

where $\mathbf{V}^{-1}$ is the inverse of the covariance matrix, $\Delta \alpha$ are the deviations of the $(N-k)$ flux factors from their nominal values, $\sigma_{\alpha_{i}}$ is the assumed $1 \sigma$ error for the $i^{\text {th }}$ flux factor and $\rho_{\alpha_{i} \alpha_{j}}$ is the correlation coefficient between the $i^{t h}$ and $j^{\text {th }}$ flux factors. The diagonal terms $(i=j)$ penalise each flux factor depending on it's deviation from the nominal value (as with the previous penalty term forms) whereas the off-diagonal $\rho_{\alpha_{i} \alpha_{j}}$ terms ensure that there are no large deviations between adjacent flux factors and hence no discontinuous and unphysical 'jumps' introduced to the true neutrino energy spectrum. Unfortunately there is currently no obvious way to decide what the correlations between the various true neutrino energy bins (which result from the a priori errors on the flux prediction) should be and so a $7 \times 7$ matrix of correlation coefficients is put in by hand and is given by:

$$
\boldsymbol{\rho}=\left(\begin{array}{lllllll}
1.0 & 0.5 & 0.0 & 0.0 & 0.0 & 0.0 & 0.0 \\
0.5 & 1.0 & 0.5 & 0.0 & 0.0 & 0.0 & 0.0 \\
0.0 & 0.5 & 1.0 & 0.5 & 0.0 & 0.0 & 0.0 \\
0.0 & 0.0 & 0.5 & 1.0 & 0.5 & 0.0 & 0.0 \\
0.0 & 0.0 & 0.0 & 0.5 & 1.0 & 0.5 & 0.0 \\
0.0 & 0.0 & 0.0 & 0.0 & 0.5 & 1.0 & 0.5 \\
0.0 & 0.0 & 0.0 & 0.0 & 0.0 & 0.5 & 1.0
\end{array}\right)
$$

This matrix of correlation coefficients is based on intuition and is designed to only penalise deviations in adjacent flux factors with the remaining correlations set to zero. A 
number of alternate matrices were considered although the constraint that the matrix be invertible (as given by equation 88) means that it can be difficult to chose appropriate values for all of the 42 off-diagonal correlations. Similar forms to that shown in equation 89 where considered but with the adjacent correlations set to 0.25 and 0.75 and almost identical results were returned by example fits using each of these choices. It seems that the fits are not very sensitive to the actual value of the adjacent flux factor correlations used but the fits must include some way to penalise unphysical warpings of the true neutrino energy spectrum and so the matrix given above was used to construct the penalty term for fits involving the flux factors. Most of the mock data studies, to be presented in the subsequent sections, are based on shape-only fits to just the $Q_{Q E L}^{2}$ distribution and do not include the flux factors because, in this case, the weak dependance of $Q_{Q E L}^{2}$ on $E_{v}^{Q E L}$ means that this fit configuration is not very sensitive to the incident neutrino flux.

The following section will discuss in more detail the various possible fit configurations and their advantages and disadvantages with respect to the systematic parameters mentioned above.

\subsection{Fit Confi gurations}

The fit is highly configurable and the following list discusses a number of the more important options:

- Fit type: the fit can be configured to use any permutation of $Q_{Q E L}^{2}$ and/or $E_{v}^{Q E L}$ with each distribution normalised by either area (equal integrated histogram bin contents and hence a shape-only fit) or POT (absolute normalisation and hence a shape and rate fit). Many of the earlier measurements of $M_{A}^{Q E L}$ used shape-only fits to the $Q_{Q E L}^{2}$ distribution because this dramatically reduces the impact of a number of the systematic parameters. The above section showed that only $M_{A}^{Q E L}$ itself and the muon energy scale parameter had a significant effect on the shape of the $Q_{Q E L}^{2}$ distribution. Figure 75 shows that the mean $Q^{2}$ for QEL-like events is fairly flat as a function of neutrino energy, except at the lowest energies, and reinforces the statement that the shape of the $Q^{2}$ distribution is not very sensitive to the underlying neutrino flux. This fit configuration does have the least sensitivity in terms of the amount of information it uses but if, for example, the flux 
were a significant systematic uncertainty then a shape-only fit to $Q_{Q E L}^{2}$ could provide the smallest error on the fitted $M_{A}^{Q E L}$.
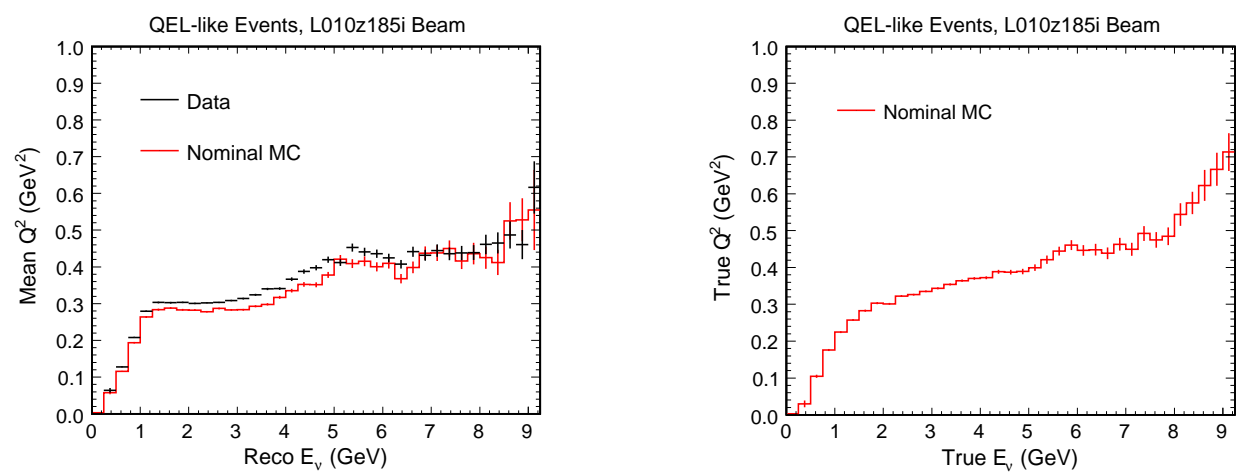

Figure 75: Mean reconstructed $Q^{2}$ in slices of reconstructed neutrino energy for QEL-like events containing stopping muons in data and nominal MC (left-hand figure) and mean true $Q^{2}$ in slices of true neutrino energy (right-hand figure). The figures show that for large parts of the reconstructed neutrino energy spectrum the $Q^{2}$ distribution does not depend strongly of the neutrino energy, in particular in the peak of the QEL-like sample neutrino energy spectrum from 1.5 to $4 \mathrm{GeV}$. The difference in shape between the nominal MC in the left-hand and right-hand figures is a result of the detector resolution for $Q^{2}$ and $E_{\mathrm{v}}$.

The $E_{\mathrm{v}}^{Q E L}$ distribution would not normally be fitted alone, because a large part of the sensitivity to $M_{A}^{Q E L}$ comes from the shape of the $Q_{Q E L}^{2}$ distribution, but a shape and normalisation fit to both $Q_{Q E L}^{2}$ and $E_{\mathrm{v}}^{Q E L}$ could be advantageous. In this configuration all the systematic parameters are important but it allows for a much better way to try to address the flux uncertainty as well as using the maximum information available. Another benefit of this combined fit is that systematic uncertainties are better constrained by the use of two distributions, in particular the muon energy scale parameter is heavily constrained by the peak of the $E_{\mathrm{v}}^{Q E L}$ distribution.

- Range in $\boldsymbol{Q}_{Q E L}^{2}$ : the fit can have any range (and indeed binning) in $Q_{Q E L}^{2}$ and for many of the mock data studies to be presented in this chapter events with $Q_{Q E L}^{2}$ values down to zero have been considered. However, the low $Q_{Q E L}^{2}$ regime $\left(<0.2 \mathrm{GeV}^{2}\right)$ is the most affected by the mis-modelling of nuclear effects and so when fitting the ND data this part of the distribution is likely to cause problems for the fit. The binning of $Q_{Q E L}^{2}$ distribution 
will be driven by the resolution studies presented at the end of the last chapter as well as by the numbers of events contained in these bins. It is worth noting that only events with neutrino energies that are reconstructed in a user-defined range will contribute to the $Q_{Q E L}^{2}$ distributions.

- Range in $\boldsymbol{E}_{\mathrm{v}}^{Q E L}$ : the fit can also have any range or binning in $E_{\mathrm{v}}^{Q E L}$. When only fitting the $Q_{Q E L}^{2}$ distribution this range can be changed to allow an extraction of $M_{A}^{Q E L}$ in a number of independant slices of neutrino energy. Nominally, when filling the $E_{\mathrm{v}}^{Q E L}$ distribution, only events where $Q_{Q E L}^{2}$ is reconstructed inside the range of the $Q_{Q E L}^{2}$ distribution to be used in the fit will contribute to the neutrino energy distribution. However, a further possibility in the fit is to set the allowed range in $Q_{Q E L}^{2}$, for events to contribute to the $E_{\mathrm{v}}^{Q E L}$ distribution, independantly from the actual range of the fitted $Q_{Q E L}^{2}$ distribution. This option allows the low $Q_{Q E L}^{2}$ events, where nuclear effect mis-modelling is likely, to be ignored for the purposes of fitting $Q_{Q E L}^{2}$ but to be included when filling the $E_{\mathrm{v}}^{Q E L}$ distribution. This setup is valid because low $Q_{Q E L}^{2}$ nuclear effects are not expected to represent a large uncertainty on the reconstruction of the neutrino energy, which is dominated by the reconstructed muon.

\subsection{Fit Convergence Problems}

Early tests of the fit revealed that it was highly susceptible to non-convergence in MINUIT and that this problem was found to be related to a number of effects that introduce fine-scale discontinuities to the $\chi^{2}$-surface and stem from the fact that the size of the MC sample used is not infinite:

- Hadronic energy offset parameter: changes to this systematic parameter result in different numbers of events passing the QEL-like sample hadronic energy cut. The finite size of the MC sample means that very small changes to this parameter between different iterations of a fit will result in a discontinous number of events being present in the fitted distributions. For example a shift of $+x \mathrm{MeV}$ may result in $y$ fewer events being present in the QEL-like sample but a shift of $+2 x \mathrm{MeV}$ may not necessarily result in $2 y$ fewer events. 
- Muon energy scale parameter: changes to the muon energy will move events between bins in both the $Q_{Q E L}^{2}$ and $E_{\mathrm{v}}^{Q E L}$ distributions and again very small changes to the parameter will induce discontinous numbers of events in certain bins and hence introduce discontinuities into the fit $\chi^{2}$ surface.

- Ranges of fitted distributions: another discontinuity can be introduced at the boundaries of the distributions where events could be shifted either into or out of the fitted range as a result of changes to the systematic parameters. This effect can be reduced by ensuring that the boundaries occur at the tails of the distributions where there are fewer events. This problem is most noticable when fitting in a number of slices of neutrino energy because slices with boundaries in the peak of the energy spectrum are likely to experience large numbers of events moving into or out of the fitted range.

Figure 76 shows an example of a discontinuous $\chi^{2}$ surface from a fit. These problems not only cause MINUIT to find a number of local minima and fail to converge but also invalidate the errors it calculates for the fitted parameters and the correlations between them. A method has been developed to artifically smooth the $\chi^{2}$ surface by applying a smearing to some of the kinematic quantities of individual MC events and this method allows the numerical minimisation to converge.

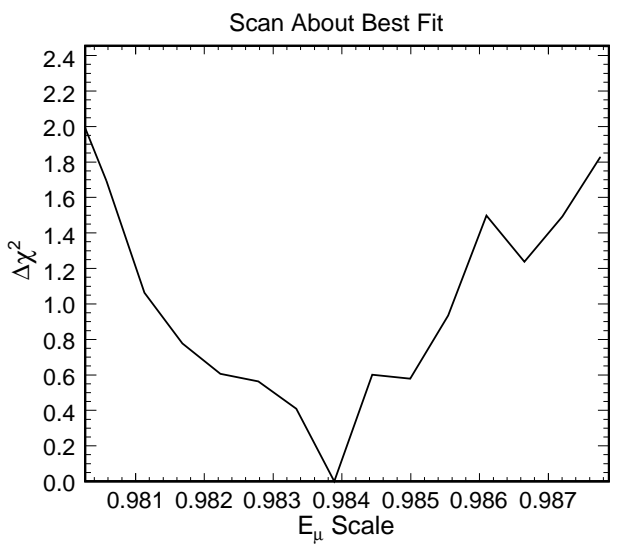

Figure 76: Example of a discontinuous $\chi^{2}$ surface. The figure shows a projection of $\Delta \chi^{2}$ as a function of the muon energy scale parameter value. 


\subsubsection{Convergence with MC Smearing}

The smearing procedure is applied to individual events in the MC and replaces the discrete value of a certain kinematic variable for each event with a Gaussian distribution centred at that value and with a certain width. As such each MC event will then have a contribution to a number of bins in that kinematic distribution, although in practice the smearing widths are so small that only events near to bin edges or cut boundaries are smeared into multiple bins. A schematic of the smearing procedure is shown in figure 77. It should also be noted that the Gaussian widths are chosen such that the resulting distortions in the kinematic distributions are negligible. Smearing is applied to both of the possible fitted variables; the reconstructed $Q_{Q E L}^{2}$ and $E_{\mathrm{v}}^{Q E L}$ as well as to the reconstructed hadronic shower energy.


Figure 77: Schematic of MC smearing procedure. The left-hand figure shows a MC event with a weight of 1 at value 1 that is replaced with a Gaussian of width $\sigma$ and area equal to the weight. In the middle figure 2 MC events are shown, with weights of 1 and 0.8 , and each event is replaced by a Gaussian of width $\sigma$ and area equal to the event weight. The right-hand figure illustrates how the kinematic variable distribution is built from the individual MC event Gaussians.

- $Q_{Q E L}^{2}$ smearing: both Gaussian widths that evolve according to the detector resolution (as defined in the previous chapter) and constant Gaussian widths of between 1 and $50 \mathrm{MeV}^{2}$ have been considered. The area under the Gaussian for each MC event is constructed to be equal to the weight for that event which is a combination of the beam and hadron production tuning weights (if they are chosen to be applied) and the weights coming from the fit parameter values. If the Gaussian distribution for an event has a contribution that has been smeared into the un-physical negative $Q_{Q E L}^{2}$ region then this 
contribution is added back to the positive $Q_{Q E L}^{2}$ part of the Gaussian distribution in such a way as to preserve both the event weight and the Gaussian width. The result is that events near bin edges and distribution boundaries in $Q_{Q E L}^{2}$ after smearing contribute to multiple bins and in this way differences between bin and sample contents, coming from the slightly different fit parameter values used in different iterations of the fit, can be smeared out to yield a smooth and continuous $\chi^{2}$-surface.

- $E_{\mathrm{v}}^{Q E L}$ smearing: this smearing is designed to smooth the $\chi^{2}$ surface in the same way as the $Q_{Q E L}^{2}$ smearing and proceeds in the same manner. Again both Gaussian widths that evolve according to the detector resolution and constant Gaussian widths (of between 1 and $100 \mathrm{MeV}$ ) have been considered.

- $\boldsymbol{E}_{\text {had }}$ smearing: the MC event Gaussian in hadronic energy is constructed with an area equal to unity and then the fraction of the event that passes the QEL-like sample hadronic energy cut is propagated as an event weight through the rest of the analysis chain. Constant Gaussian widths of between 1 and $50 \mathrm{MeV}$ have been considered. This smearing process ensures that the number of events that pass into the QEL-like sample changes in a continuous way under very small changes to the hadronic energy offset systematic parameter and results in a smooth and continuous $\chi^{2}$-surface.

The criteria for choosing the values of the widths of the Gaussian distributions for the three variables to be smeared were that they should allow for fit convergence with a smooth $\chi^{2}$ surface whilst preserving the underlying shapes of the distributions. This second criterion ensures that the smearing process does not warp the fitted $Q_{Q E L}^{2}$ and $E_{\mathrm{v}}^{Q E L}$ distributions as such a warping would bias the fitted $M_{A}^{Q E L}$ value.

It was found that these criteria were satisfied by Gaussian smearing widths of $5 \mathrm{MeV}^{2}$ in $Q_{Q E L}^{2}, 10 \mathrm{MeV}$ in $E_{\mathrm{v}}^{Q E L}$ and $2.5 \mathrm{MeV}$ in $E_{\text {had }}$. These widths were the smallest values that resulted in all the test fits converging and figure 78 shows an example of the smooth onedimensional projections of the $\Delta \chi^{2}$-surface along the muon energy scale and hadronic energy offset systematic parameters for one of these test fits. Figure 79 shows the ratios of the smeared 
MC to the unsmeared MC and illustrates that the smearing process has a neglibible sub-1\% effect on the shapes and normalisation of the fitted distibutions.
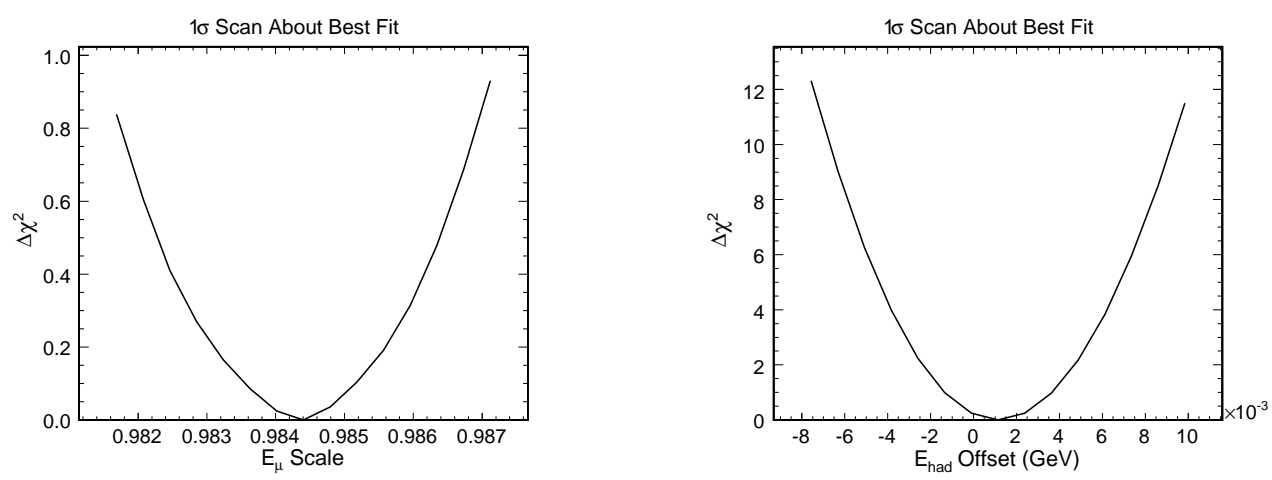

Figure 78: Projections of $\Delta \chi^{2}$ for the two main problematic fit parameters after a fit that used the MC smearing of the three kinematic variables. These projections are now smooth and continuous (compare to the dis-continuous $\Delta \chi^{2}$ surface shown in figure 76 ) and resulted from a well behaved and converged fit.
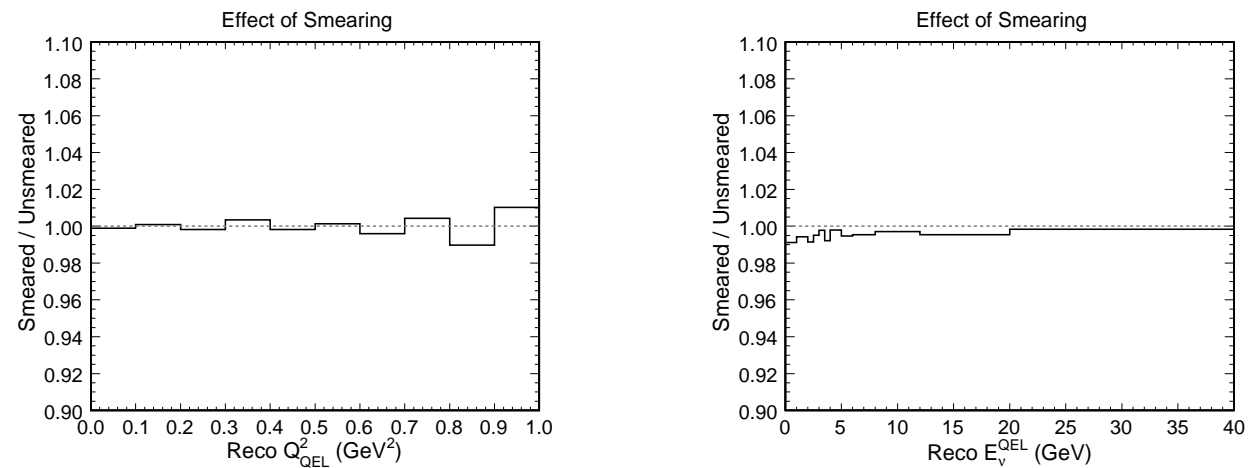

Figure 79: Ratios of smeared to unsmeared MC. The smearing can be seen to be having less than a $1 \%$ effect over the majority of the two possible fit distributions and in particular that their shapes are entirely consistent. 


\subsection{Headline Fit Confi gurations}

This section will detail the two main mock data fit configurations (as briefly mentioned in section 7.3) and present example results from a fit with each. Firstly there are a number of fit configuration options that are common to both these mock data headline fits:

- Hadronic energy cut: the nominal hadronic energy cut that defines the QEL-like sample requires $E_{\text {had }}<250 \mathrm{MeV}$. This value can be changed in order to study the robustness of the fit and to consider QEL-like samples with higher efficiency (larger cut value) or purity (lower cut value).

- Minimised function: the nominal function to be minimised in the fits is $\chi_{M C S t a t s}^{2}$ as defined by equation 87 .

- MC samples: for these mock data fits two independant sub-samples of MC are used and they correspond to exposures of 2.26e19 POT for the MC and 2.27e19 POT for the mock data. These are substantially lower exposures than will be used for the fit to the data but do allow the fit to converge faster.

- MINUIT routines: the fits nominally use three routines inside MINUIT; SIMPLEX is called first to get close to the minimum quickly then MIGRAD is called to find the exact minimum before HESSE is called to provide a better estimate of the errors on the fitted parameter values and to estimate the correlations between the parameters. These routines will be discussed further in a following section along with an alternate error calculation using the MINOS routine.

- Smearing of MC events: in all mock data fits smearing is applied according to Gaussian widths of $5 \mathrm{MeV}^{2}$ in $Q_{Q E L}^{2}, 10 \mathrm{MeV}$ in $E_{\mathrm{v}}^{Q E L}$ and $2.5 \mathrm{MeV}$ in $E_{\text {had }}$.

The headline fit configurations are then further defined in the following sub-sections.

\subsubsection{Headline Fit Configuration I}

A shape-only fit to the $Q_{Q E L}^{2}$ distribution. In this fit only three systematic parameters are used; the muon energy and background scales and the hadronic energy offset. The incident neutrino 
flux has only a small effect on the shape of the $Q_{Q E L}^{2}$ distribution and so the flux factor parameters are not used. The understanding of the systematic uncertainties was improving while this work was under way and so the mock data studies that were performed with this fit configuration used smaller assumed $1 \sigma$ errors than those chosen for the fits to the data; $20 \mathrm{MeV}$ for the hadronic energy offset parameter (rather than $35 \mathrm{MeV}$ ) and 5\% for the background scale (rather than $10 \%$ ). The assumed $1 \sigma$ error for the muon energy scale was, however, set to it's appropriate value of $2 \%$. Due to the large amount of work required these studies have not been repeated using larger errors. However, the hadronic energy offset and background scale parameters do not have a large effect on the shape of the fitted distribution and so these smaller error values are not expected to change the conclusions of the mock data studies of the fit robustness and accuracy.

The $Q_{Q E L}^{2}$ distribution is constructed using bins of width $0.1 \mathrm{GeV}^{2}$ from $0.0 \rightarrow 1.0 \mathrm{GeV}^{2}$ and a large overflow bin from $1.0 \rightarrow 10.0 \mathrm{GeV}^{2}$. There are very few events with reconstructed $Q_{Q E L}^{2}$ above $\sim 2 \mathrm{GeV}^{2}$ however this overflow bin helps to mitigate against the discontinuities in the $\chi^{2}$-surface that can result from events migrating into and out of the fitted distributions. Events with neutrino energies from $0.0 \rightarrow 40.0 \mathrm{GeV}$ are used although the vast majority of events in the QEL-like sample have neutrino energies below $20 \mathrm{GeV}$ (the cross section for QEL scattering peaks at $\sim 1 \mathrm{GeV}$ and decreases as the incident neutrino energy rises).

\subsubsection{Headline Fit Configuration II}

A shape and normalisation fit to both the $Q_{Q E L}^{2}$ and $E_{\mathrm{v}}^{Q E L}$ distributions. In this fit a total of eleven parameters are included; the $M_{A}^{Q E L}$-scale, the three systematic parameters used in fit I and the seven flux factors which are utilised because the inclusion of the neutrino energy distribution in the fit provides some sensitivity to the incident neutrino flux. For the mock data studies performed with this fit configuration the penalty term for the flux factors only considered the diagonal elements of the covariance matrix equation and so large deviations in adjacent flux factors are not penalised. For this fit the appropriate assumed $1 \sigma$ errors (as described in the text) are used for all fit parameters.

The $Q_{Q E L}^{2}$ distribution is binned in the same way as described for fit I and the $E_{\mathrm{v}}^{Q E L}$ distribution 


\begin{tabular}{|c|c|c|c|}
\hline Fit Parameter & $\begin{array}{c}\text { Assumed 1 } \sigma \\
\text { Error }\end{array}$ & $\begin{array}{c}\text { Mock Data } \\
\text { Value }\end{array}$ & $\begin{array}{c}\text { Best Fit } \\
\text { Value }\end{array}$ \\
\hline \hline$M_{A}$-scale & Free & 0.854 & $0.851 \pm 0.037$ \\
\hline$E_{\mu}$-scale & 0.02 & 0.988 & $0.986 \pm 0.003$ \\
\hline$E_{\text {had }}$-offset $(\mathrm{GeV})$ & 0.02 & 0.010 & $0.022 \pm 0.009$ \\
\hline BG-scale & 0.05 & 1.013 & $0.993 \pm 0.042$ \\
\hline
\end{tabular}

Table 8: Summary of results for example fit I to mock data. The best fit values and symmetric errors are those returned by the HESSE routine. The best fit parameter values agree well with the randomly chosen input mock data parameter values.

has an asymmetric binning scheme that uses finer bins in the peak of the distribution and is best illustrated (see figure 82) rather than described in words. The following example results will show the distribution binnings used for these two headline fit configurations as well as demonstrating the correlations that exist between different fit parameters.

\subsubsection{Example Results for Fit I}

For the purposes of studying the fit performance the mock data sample is adjusted with a certain set of initial fit parameter values which, in the ideal case, would then be 'fitted-out' using the MC. Figure 80 shows the $Q_{Q E L}^{2}$ distribution for an example fit to the mock data and illustrates the choice of analysis binning in $Q_{Q E L}^{2}$. Table 8 shows a summary of the results of this fit and includes the best fit parameter values, the errors returned by the HESSE routine and the mock data input parameter values. The fit does a good job of extracting the input mock data parameters and results in an improvement in $\chi^{2}$ from 114.13 to 22.26 for ten degree of freedom. This number of degrees of freedom comes from the eleven bins of the fitted distribution minus the single free parameter; the $M_{A}^{Q E L}$-scale. Each of the systematic parameters consitute both a degree of freedom and also a measurement point (the assumed nominal value of the parameter) and so have zero net contribution to the total number of degrees of freedom in the fit.

Figure 81 shows two-dimensional contour plots for the $M_{A}^{Q E L}$-scale parameter versus each of the three systematic parameters. The best way to construct such two-dimensional contour plots for an $N$ parameter fit would be to minimize over the remaining $N-2$ parameters at each point on the contour. However, this procedure requires a large amount of time (with a contour 

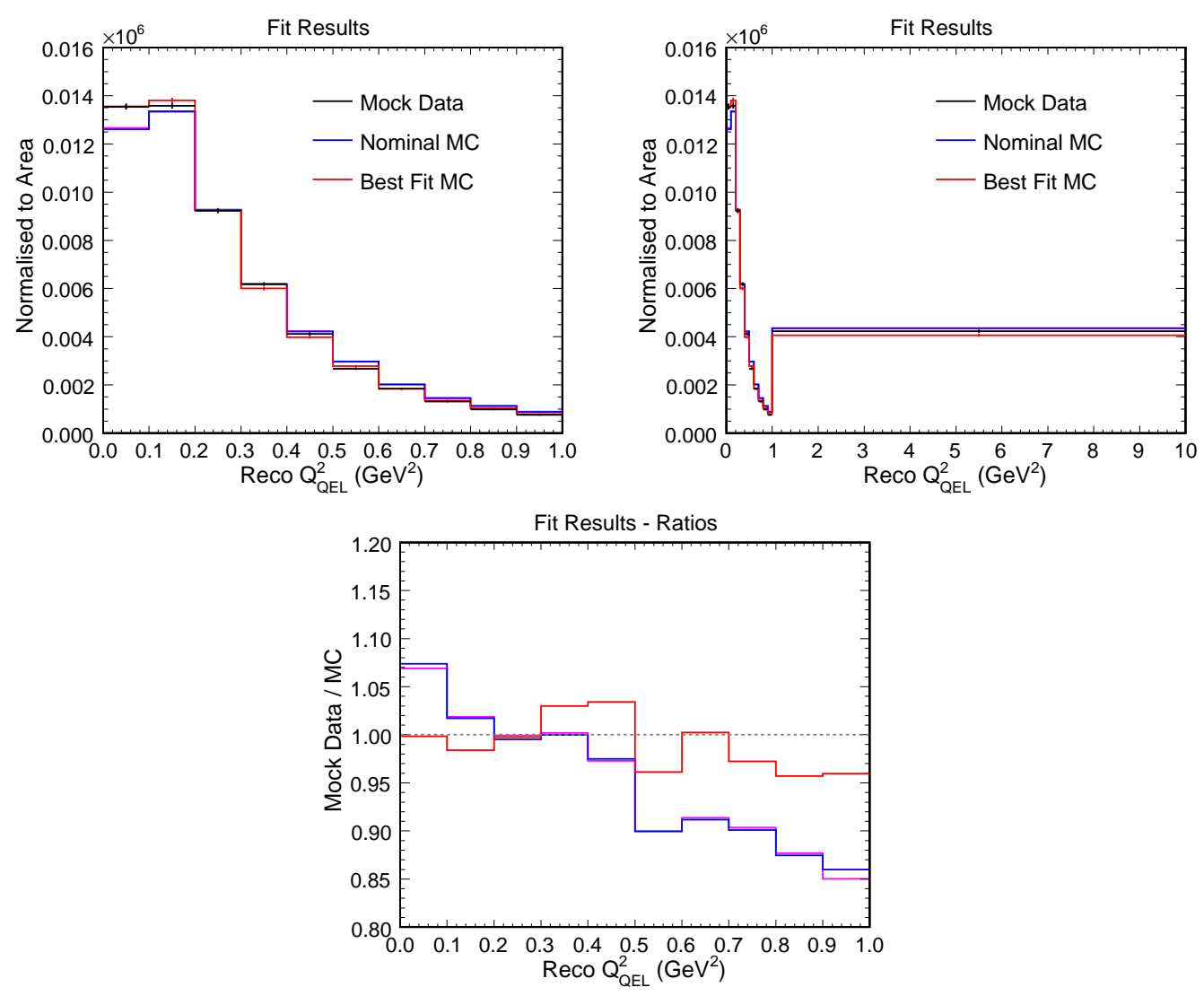

Figure 80: Example results for mock data fit I. The top right figure shows the fitted $Q_{Q E L}^{2}$ distributions whilst the top left figure shows a zoom of the region from $0.0 \rightarrow 1.0 \mathrm{GeV}^{2}$. The black histograms show the mock data, the blue histograms show the nominal MC, the pink histograms show the unsmeared nominal MC (which is almost identical to the smeared MC) and the red histograms show the best fit $M C$. The bottom plot shows the ratios of the mock data to each MC sample.

needing a minimum of about twenty points) and so these contours are not minimized at each point with respect to the parameters not shown on the axes but rather have been approximated using an elliptical equation given by:

$$
\begin{gathered}
-2 \mathscr{L}(\alpha, \beta)=-2 \mathscr{L}(\alpha, \beta)_{\text {min }}-N \\
\Rightarrow \frac{1}{1-\rho_{\alpha \beta}^{2}}\left[\left(\frac{\alpha-\hat{\alpha}}{\sigma_{\hat{\alpha}}}\right)^{2}+\left(\frac{\beta-\hat{\beta}}{\sigma_{\hat{\beta}}}\right)^{2}-2 \rho_{\alpha \beta}\left(\frac{\alpha-\hat{\alpha}}{\sigma_{\hat{\alpha}}}\right)\left(\frac{\beta-\hat{\beta}}{\sigma_{\hat{\beta}}}\right)\right]=N
\end{gathered}
$$

where $\alpha$ and $\beta$ are the two fit parameters for which the contour is to be drawn, $\rho_{\alpha \beta}$ is the 
correlation coefficient between the two parameters as returned by HESSE, $\hat{\alpha}, \hat{\beta}, \sigma_{\hat{\alpha}}, \sigma_{\hat{\beta}}$ are the best fit parameter values and errors as returned by HESSE and $N \in(2.30,6.18,11.83)$ for the $68.27 \%, 95.45 \%$ and $99.73 \%$ level contours respectively.


Figure 81: Fit contours for the $M_{A}^{Q E L}$-scale parameter versus each of the systematic parameters. The black points show the best fit values, the pink points show the initial mock data parameter values and the black, red and blue ellipses show the $1 \sigma, 2 \sigma$ and $3 \sigma$ contours respectively.

The fit parameter correlations returned by HESSE should not be considered to be accurate to more than $10-20 \%$ (this statement will be qualified in the next section) but figure 81 does illustrate the general trends in the correlations with the eccentricity of each ellipse relating to the size of the correlation and the orientation of each ellipse indicating whether the correlation is positive or negative.

As expected for fit I the hadronic energy offset and background scale parameters are not highly 


\begin{tabular}{|c|c|c|c|}
\hline Fit Parameter & $\begin{array}{c}\text { Assumed 1 } \sigma \\
\text { Error }\end{array}$ & $\begin{array}{c}\text { Mock Data } \\
\text { Value }\end{array}$ & $\begin{array}{c}\text { Best Fit } \\
\text { Value }\end{array}$ \\
\hline \hline$M_{A}$-scale & Free & 0.948 & $0.960 \pm 0.040$ \\
\hline$E_{\mu}$-scale & 0.02 & 0.998 & $0.996 \pm 0.003$ \\
\hline$E_{\text {had }}$-offset $(\mathrm{GeV})$ & 0.035 & -0.025 & $-0.028 \pm 0.009$ \\
\hline BG-scale & 0.10 & 0.992 & $0.968 \pm 0.057$ \\
\hline 0-2 GeV Flux Scale & 0.08 & 1.008 & $0.988 \pm 0.035$ \\
\hline 2-3 GeV Flux Scale & 0.08 & 0.997 & $1.029 \pm 0.031$ \\
\hline 3-4 GeV Flux Scale & 0.08 & 0.984 & $0.975 \pm 0.026$ \\
\hline 4-6 GeV Flux Scale & 0.08 & 0.963 & $1.034 \pm 0.031$ \\
\hline 6-10 GeV Flux Scale & 0.15 & 1.000 & $0.994 \pm 0.046$ \\
\hline 10-20 GeV Flux Scale & 0.15 & 1.081 & $1.085 \pm 0.045$ \\
\hline 20-40 GeV Flux Scale & 0.15 & 0.924 & $1.036 \pm 0.065$ \\
\hline
\end{tabular}

Table 9: Summary of results for example fit II to mock data. The best fit values and symmetric errors are those returned by the HESSE routine. The best fit parameter values agree well with the randomly chosen input mock data parameter values. The higher neutrino energy flux factors have larger errors both because the assumed $1 \sigma$ errors on these parameters are larger than for the lower energy flux factors but also because there are relatively few $Q E L$-like events above $\sim 10 \mathrm{GeV}$.

correlated with the $M_{A}^{Q E L}$-scale since they do not have large effects on the shape of the $Q_{Q E L}^{2}$ distribution. The muon energy scale is highly anti-correlated with the $M_{A}^{Q E L}$ scale because they have opposing effects on the $Q_{Q E L}^{2}$ distribution shape (see figures 68 and 69).

\subsubsection{Example Results for Fit II}

For this example, as before, the mock data has had randomly selected input fit parameter values applied and figure 82 shows the mock data, nominal $\mathrm{MC}$ and best fit $\mathrm{MC} Q_{Q E L}^{2}$ and $E_{\mathrm{v}}^{Q E L}$ distributions. Table 9 summarises the results for this fit and shows a good agreement between the input mock data fit parameter values and the resulting best fit point. In this fit the $\chi^{2}$ was reduced from 112.90 to 17.77 for 23 degrees of freedom. This number of degrees of freedom is constructed from the eleven bins of the $Q_{Q E L}^{2}$ distribution plus the thirteen bins of the $E_{\mathrm{v}}^{Q E L}$ distribution minus the single free parameter; the $M_{Q E L}^{A}$-scale. As before, the net contribution from the systematic parameters to the number of degrees of freedom in the fit is zero. 



Figure 82: Example results for mock data fit II. The left-hand plots show the $Q_{Q E L}^{2}$ distributions and the ratios of the mock data to the various MC samples and are all zoomed such that the overflow bin from $1.0 \rightarrow 10.0 \mathrm{GeV}^{2}$ is not visible. The right-hand plots show the neutrino energy distributions and illustrate the choice of binning used in the fits. The peak region uses the smallest bin widths, the widths increase as the numbers of QEL-like events in a given bin decreases and in no region do the widths become smaller than the expected resolution for the neutrino energy. The bins were also chosen such that they do not completely overlap with the definition of the flux factors.

It can be seen from tables 8 and 9 that the HESSE errors for the parameters are similar between fits I and II which is contrary to the expectation that the errors should be smaller for fit II (since it uses extra $E_{\mathrm{v}}^{Q E L}$ and normalisation information). However, the larger number of fit paramaters used in fit II will introduce many more correlations into the fit with the result that the fit sensitivity to the parameters will be degraded. The similarity in the parameter errors for these two headline fit configurations suggests that, assuming the quoted exposures of POT, they can both achieve similar sensitivities for $M_{A}^{Q E L}$ and provides additional justification for the continued use of both. 
Figure 83 shows two-dimensional contour plots for the $M_{A}^{Q E L}$-scale parameter versus each of the three systematic parameters used in fit I and again uses the elliptical approximation as defined by equation 91 . For fit II there is still a fairly strong anti-correlation between the $M_{A}^{Q E L}$ scale and $E_{\mu}$-scale parameters but there are now also stronger correlations between the other parameters, in particular the $M_{A}^{Q E L}$-scale and the $E_{\text {had }}$-offset. This strong positive correlation can be understood as the effect of normalisation in the fit; increases to $M_{A}^{Q E L}$ will give larger weights to the events in the QEL-like sample whereas increases in the hadronic energy will move events out of the QEL-like sample. With eleven fit parameters there are a large number of correlations to consider and further discussion of these correlations can be found in the following chapter when discussing the results of fits to the data.
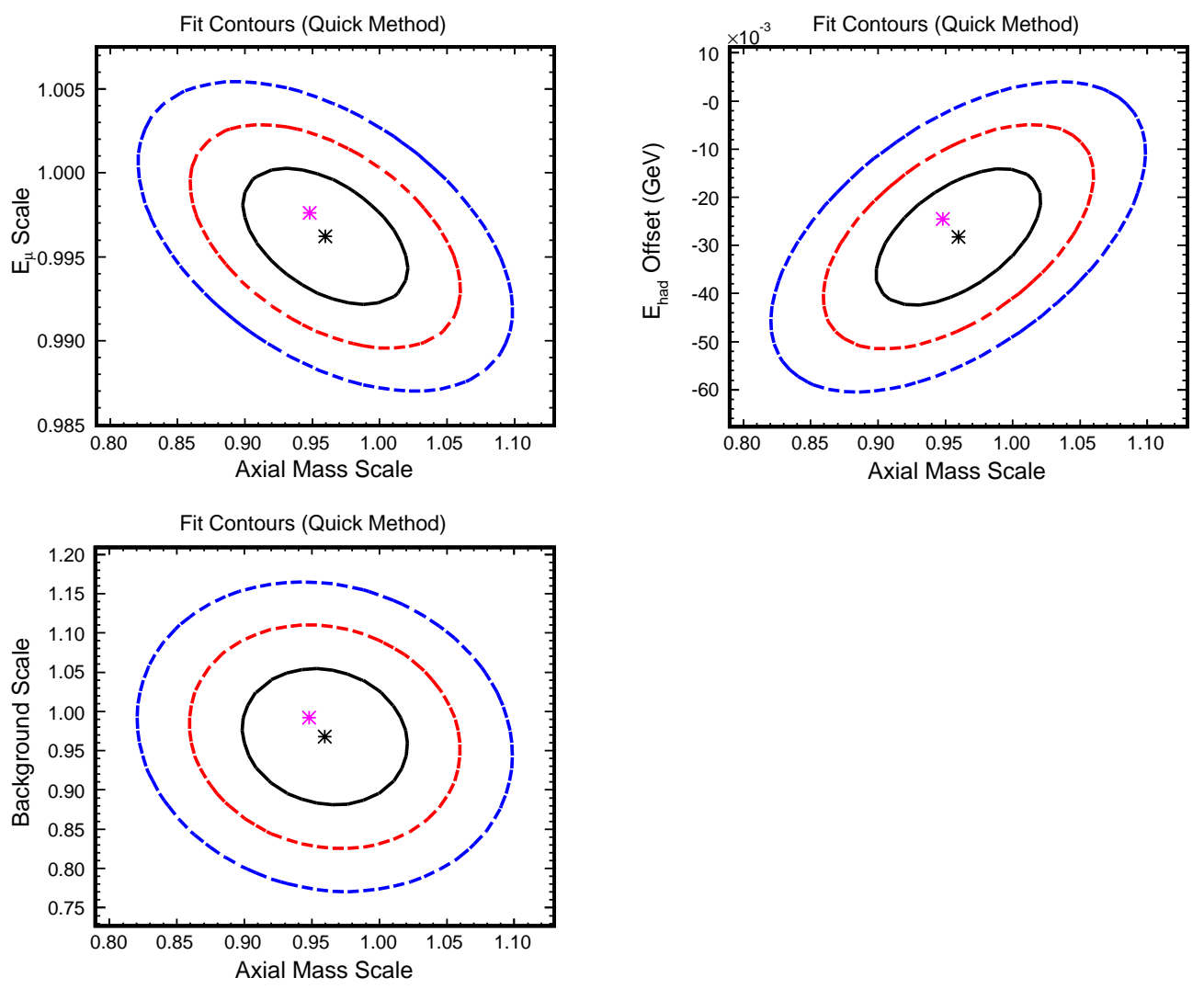

Figure 83: Fit contours for the $M_{A}^{Q E L}$-scale parameter versus the non-flux factor systematic parameters. The black points show the best fit values, the pink points show the initial mock data parameter values and the black, red and blue ellipses show the $1 \sigma, 2 \sigma$ and $3 \sigma$ contours respectively. 


\subsection{Studying the Fit}

This section presents results from a number of mock data studies aimed at quantifiying the robustness and sensitivity of the fit method. These studies were performed by holding all fit configuration choices constant apart from the option in question and then performing fits with a number of different values of this option. Most of the studies were performed with fit I (which should be assumed unless stated otherwise) since it converges far quicker than the more complicated fit II and considered two very different choices of input mock data fit parameter values in order to check for consistency between the results. The following list details checks that were made on the robustness of the fitting method:

- $-2 \mathscr{L}$ v.s. $\chi^{2}$ v.s. $\chi_{\text {MCstats }}^{2}$ : in this study fits were performed using the three different functions defined earlier in equations 85-87. All the fits converged to almost identical parameter values with the best fit $-2 \mathscr{L}$ and $\chi^{2}$ values being almost exactly the same (as expected given the relatively large MC sample statistics). The best fit $\chi_{M C s t a t s}^{2}$ value was about half the size as that given by the other functions which is also expected given that the mock data and MC samples used have almost identical exposures.

- MINUIT initial parameter values: MINUIT requires the user to choose starting values for each parameter to be included in the fit and the purpose of this study was to check that moving the parameter start values, in particular both towards and away from the truth input mock data parameter values, does not influence the results of the fit. Fits were performed with many permutations of parameter start values and the best fit results were consistent at the $0.01 \%$ level.

- MINUIT initial parameter errors: MINUIT also requires the user to choose the initial errors to be assigned to each fit parameter. These errors are used as step sizes in MINUIT's first iteration (in each parameter) and for all subsequent iterations the step sizes are automatically decided inside MINUIT. In this study fits were run with many permutations of initial parameter errors to check that this choice did not influence the results. As with the initial parameter value study all results were consistent at the $0.01 \%$ level.

- MINUIT parameter allowed ranges: MINUIT allows the user to set boundaries for 
each fit parameter which can be useful for problems with unphysical regions of parameter space. The purpose of this study was to check that the chosen range for the fit parameters did not affect the fit results. Fits were performed to many permutations of parameter allowed ranges but did not allow these ranges to go unphysical or very tight about the truth mock data input values. The fit results were once again consistent at the $0.01 \%$ level.

- MINUIT strategy choice: some MINUIT routines, such as MIGRAD, are dependant on the user inputted choice of 'strategy'. The strategy integer governs the way these routines calculate the first and second derivatives of the function to be minimized and can take values of:

- 0: Forces convergence to be fast at the expense of reliability.

- 1: The routine performs it's own internal balancing of speed and reliability.

- 2: Forces convergence to be accurate at the expense of time taken.

Fits were performed with all choices of strategy to check what effect it had on the fit results. The results were almost identical and the default choice of strategy $=1$ has been chosen for use in the fits.

- Iterations of MINUIT routines: nominally the fits are performed with an initial call to the minimization routine S IMP LEX which takes MINUIT to the vicinity of the minimum in a timely fashion. Next the more rigourous minimization routine MIGRAD is called to focus in on the true minimum and finally the HESSE routine is called which calculates the matrix of second derivatives of the minimized function in order to provide better estimates of the parameter errors and correlations. As such HESSE assumes a symmetry in the minimized function and only provides symmetric error estimates for each parameter. A further step, that has not been taken for many of these fit studies due to time considerations, is to call the MINOS routine which provides a much better estimate of the parameter errors. MINOS does not use the matrix of second derivatives directly but rather steps out from the minimum in parameter space and can provide asymmetric estimates of the parameter errors. 
The purpose of this study was to iteratively call the routines MIGRAD, HESSE and MINOS and compare the best fit parameter values, errors and correlations at each step. Several fits were performed with multiple iterations of these three routines (after the initial S IMP LEX call) and the results show that:

- The second call to MIGRAD finds a slighlty better minimum than the first call. This is due to the extra call to HESSE which forces a calculation of the matrix of second derivatives and aids the second MIGRAD iteration. All further iterations of MIGRAD find the same minimum.

- Each iteration of HESSE found different values for the fit parameter errors and correlation coefficients with differences of up to $20 \%$.

- Each iteration of MINOS found almost identical fit parameter errors. The asymmetric errors given by MINOS were generally slightly larger than those returned by HESSE.

The conclusions from this study are that HESSE should only ever be considered as an approximate estimate of the fit parameter errors and correlations (as mentioned previously) and that MINOS should be called to get a good estimate of these errors. The only drawback for MINOS is that it is computationally intensive and, for more than a few fit parameters, can take a long time. For this reason the majority of fits to both mock data and real data do not use the MINOS routine and quote errors and correlations from HESSE with the caveat that these are only an estimate.

- Varying the $\mathbf{E}_{\text {had }}$ cut: the nominal QEL-like sample selection cut is at $E_{\text {had }}<250 \mathrm{MeV}$ and the purpose of this study was to vary this cut value in both mock data and MC to check the resulting fitted parameter values. Figure 84 shows a compilation of the results for fits with seven different values of the hadronic energy cut and for two sets of input mock data parameter values. This study was performed with a modified configuration I; the fit considers both the shape and normalisation of the $Q_{Q E L}^{2}$ distribution rather than just it's shape. 

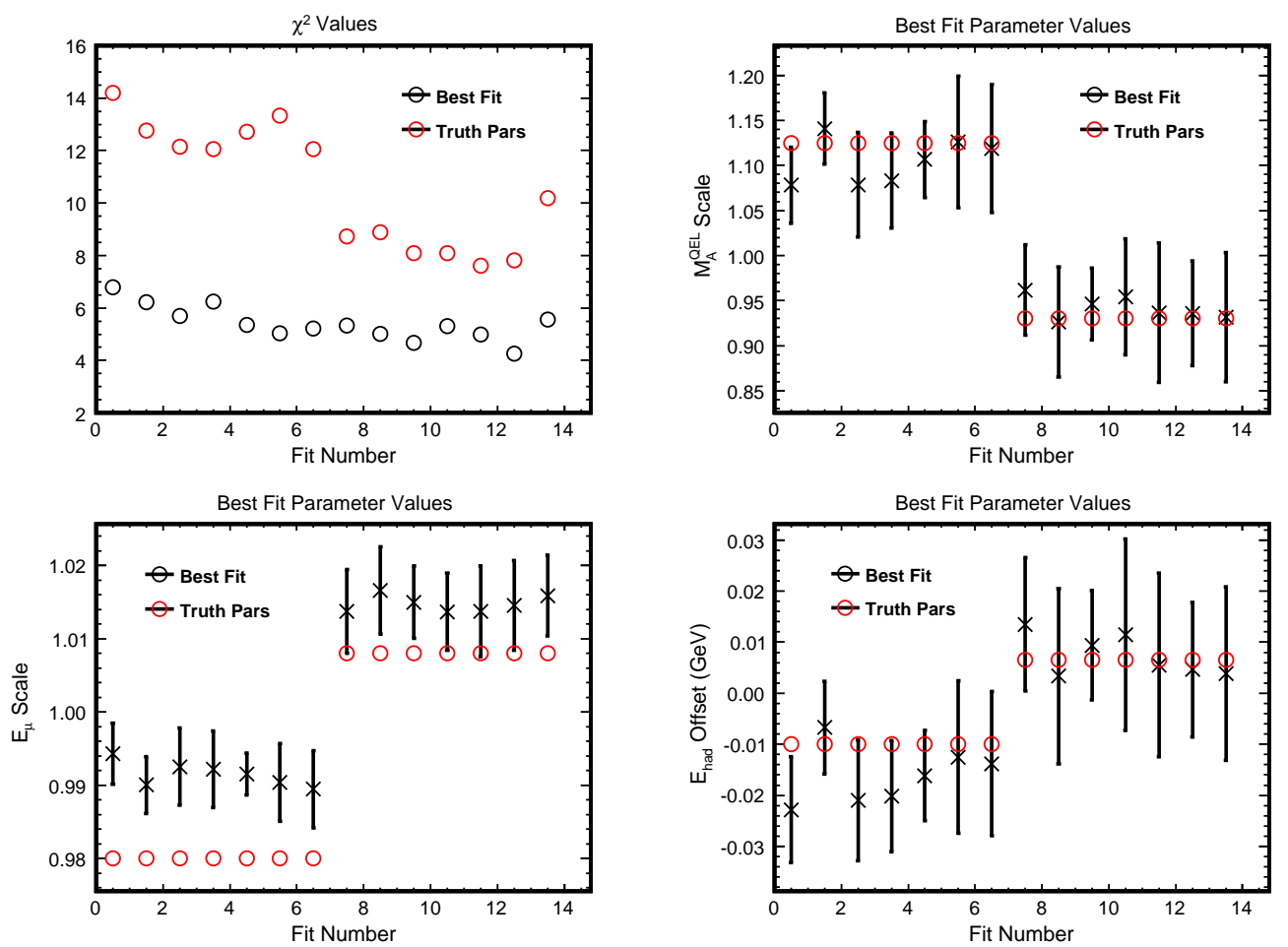

Figure 84: Fit results with a variety of different $E_{\text {had }}$ cut values. For each of the 2 sets of mock data fit parameters there are 7 fits, indexed on the $x$-axes, corresponding to cut values of 200,225,240,250,260,275

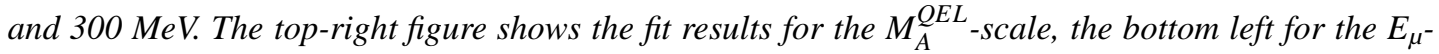
scale and the bottom right for the $E_{\text {had }}$-offset. Error bars for each parameter correspond to the symmetric errors reported by HESSE and are shown in black whilst the input mock data parameter values are shown by the open red circles. The top left figure shows the $\chi_{M C S t a t s}^{2}$ for the best fit MC and for the MC when the true mock data input parameters have been applied.

The top left part of figure 84 shows that the best fit points found in each case do have a better $\chi^{2}$ value than when the true mock data input parameters are used. This is because the mock data and MC samples are statistically independant and there are residual differences in the $Q_{Q E L}^{2}$ distributions for the two samples that the fit will try to accomodate (as well as trying to fit out the input mock data parameter values). For each set of seven fits the remaining parts of figure 84 show that the best fit points for each parameter are very consistent within the returned HESSE errors. The only exception is fit 1 which shows a shift in both the $M_{A}$-scale and the $E_{\text {had }}$-offset relative to the surrounding fits. This is not a bad fit but rather a reflection of the fact that in the shape and normalisation fit these two parameters are highly correlated; an increase in $M_{A}$ will weight up events in the QEL- 
like sample whereas an increase in the hadronic energy will mean that fewer events pass into the QEL-like sample. There is an apparent bias in the fitted parameter values which is particularly evident for the best fit $E_{\mu}$-scales.

- Apparent bias studies: as was mentioned above there are statistical differences in the $Q_{Q E L}^{2}$ distributions for the mock data and MC samples which the fit will have to accomodate on top of any adjustments of the mock data sample generated by the initial fit parameter values. These residual differences result in a bias in the best fit parameters when compared to the input mock data parameter values. Fits were performed where each of the mock data and MC samples was broken down into two further sub-samples. One of these four sub-samples was used as MC and the remaining three as statistically independant sets of mock data. Fits to these separate mock data samples did not show a consistent bias indicating that the apparent bias is not an inherent feature of the fit method but rather due to these small statistical differences in the $Q_{Q E L}^{2}$ distribution bin contents.

As a further check (and to get away from the problem of parameter correlations complicating the issue) shape-only fits to $Q_{Q E L}^{2}$ were performed which included only the $M_{A}^{Q E L}$-scale parameter. In the study presented here two of the four statistically independant samples of MC were each used as both mock data and MC in the fits. The first MC sample was used as mock data (with the second used as MC) and twenty fits were performed to randomly generated (between $\pm 20 \%$ ) mock data $M_{A}^{Q E L}$-scale initial values. A consistent bias in the fit results was observed. Following this the second MC sample was then used as mock data (with the first used as MC) and a further twenty fits were performed to randomly generated $M_{A}^{Q E L}$-scale initial values. Again a consistent bias was observed in the results but this bias was opposite to that observed in the first set of twenty fits. This reversal of the bias under a reversal of the two MC samples confirms that the fit procedure is not inherently biased but rather that the fit is accomodating the statistical differences between the $Q_{Q E L}^{2}$ distributions of the two MC samples. Figure 85 shows the results of the twenty fits to randomly generated (between $\pm 20 \%$ ) mock data $M_{A}^{Q E L}$-scale initial values and also the results of the twenty fits with the MC samples reversed. Fur- 
ther details of the full set of apparent fit bias studies, including fits using the remaining two statistically independant MC samples as well as figures illustrating the statistical differences between the various samples, can be found in appendix A.
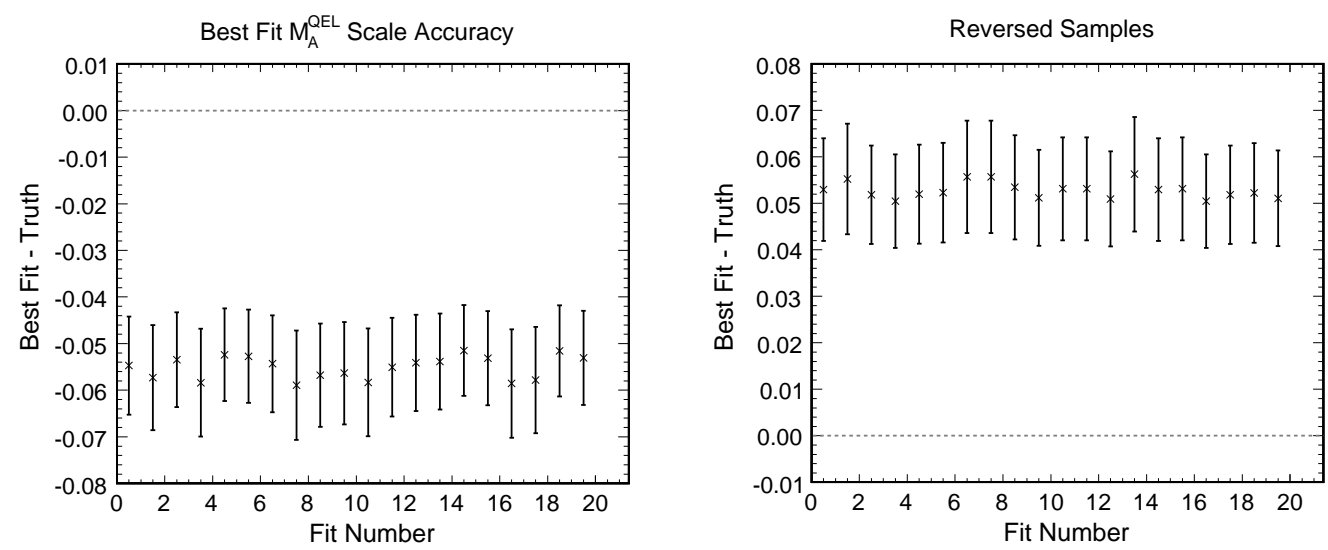

Figure 85: Best fit minus truth $M_{A}^{Q E L}$-scale values for 20 single parameter fits (indexed on the $x$-axis) to a mock data sample with randomly generated $M_{A}^{Q E L}$-scale input values and 20 fits with the mock data and MC samples reversed.

- Range and binning of the $\mathbf{Q}_{\mathrm{QEL}}^{2}$ distribution: the purpose of this study was to see what effect the choice of binning and range in $Q_{Q E L}^{2}$ has upon the fit results. Again a modified version of fit I that fits the $Q_{Q E L}^{2}$ distribution using both shape and normalisation was used for this study and a variety of ranges and binning in $Q_{Q E L}^{2}$ were considered. Figure 86 summarises the results of these mock data fits.

Figure 86 shows that the best fit parameters are consistent within their errors and that the fit is robust to these changes. It also shows that removal of the low $Q_{Q E L}^{2}$ region (where nuclear effect mis-modelling is most likely) does not change the fitted parameter values in these shape and normalization fits. Fits were also performed with the same set of ranges and binning but using a different set of input mock data parameters and these fits confirmed the conclusions drawn here. 

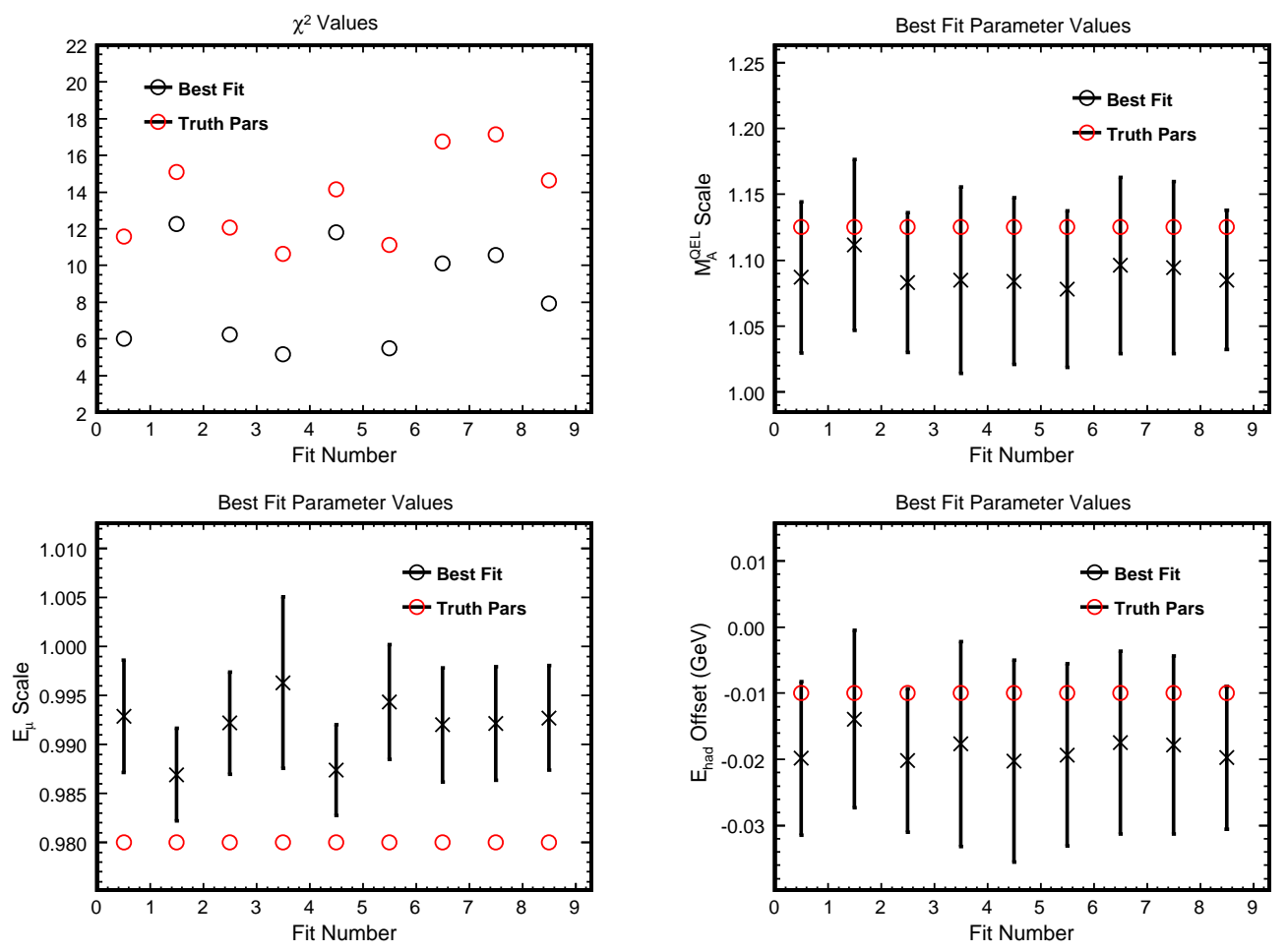

Figure 86: Mock data fit results with a variety of ranges and binning in $Q_{Q E L}^{2}$. The fit numbers are indexed along the $x$-axes, open red circles correspond to the mock data truth parameters and the black points show the best fit values along with the symmetric errors returned by HESSE. Fit 0 uses 10 symmetric bins from 0.0 to $1.0 \mathrm{GeV}^{2}$ whilst fit 1 extends this to 15 bins from 0.0 to $1.5 \mathrm{GeV}^{2}$ and fit 2 uses the nominal binning scheme of 10 bins from 0.0 to $1.0 \mathrm{GeV}^{2}$ with an overflow bin from 1.0 to $10.0 \mathrm{GeV}^{2}$. Fits 3,4 and 5 use the same binning scheme as fits 0,1 and 2 (respectively) but with the low $Q_{Q E L}^{2}$ region from 0.0 to $0.2 \mathrm{GeV}^{2}$ removed. Fits 6,7 and 8 all use a range from 0.0 to $1.0 \mathrm{GeV}^{2}$ with an overflow bin up to $10.0 \mathrm{GeV}^{2}$ but use progressively smaller bin widths for the region below $0.3 \mathrm{GeV}^{2}$ where the majority of the shape information is contained. 
To quantify the sensitivity of the fit, to $M_{A}^{Q E L}$ and the systematic parameters, large numbers of fits to the mock data sample were run in a number of fit configurations and in each fit the mock data input parameter values were sampled uniformly from between the $\pm 1 \sigma$ errors for each parameter (and between $\pm 20 \%$ for the $M_{A}^{Q E L}$-scale). After all of the fits in each configuration had been run the widths of the distributions of best fit value minus truth values for each parameter provide an estimate of the sensitivity of that fit configuration to that parameter.

Figure 87 shows best fit minus truth values for the four non-flux factor fit parameters from the results of 30 configuration II fits to the mock data sample where in each fit the mock data input parameters were chosen at random as decsribed above. Figure 88 shows projections of best fit minus truth values for each of the parameters shown in figure 87 and quotes both mean and RMS values for these projections. The apparent bias that can be seen in the means was discussed above and should not be thought of as an intrinsic bias of the fit but the RMS values do give an estimate of the fit sensitivity to each parameter.

For the first fit shown in figure 87 the initial mock data fit parameter values were set to the nominal values of the parameters and so the best fit results are directly related to accomodating any statistical differences between the $Q_{Q E L}^{2}$ distributions of the mock data and MC. As such the subsequent fits to randomly generated initial mock data parameter values might be expected to exhibit similar biases in the best fit parameter values as the first fit (with some spread being caused by the fit parameter correlations). The fitted zeroth order polynominals shown in figure 87 agree very well with the results of the first fit confirming that, on average, the bias in the subsequent fits is consistent with that displayed by the fit to the nominal mock data.

The systematic parameters are constrained by the inclusion of the penalty term in the $\chi^{2}$ but the $M_{A}^{Q E L}$-scale is a free parameter in the fits and it's distribution of best fit minus truth values is measured to have an RMS of $\sim 0.053$. The size of this RMS is comparable to the quoted errors of many of the previous measurements of $M_{A}^{Q E L}$ (see chapter 3) and only corresponds to an exposure about one tenth of the size of the current ND data sample. It is not obvious how this RMS will scale with POT exposure but it's value, along with the parameter errors quoted from the example fit II shown in the previous section, illustrate that a MINOS $M_{A}^{Q E L}$ measurement 

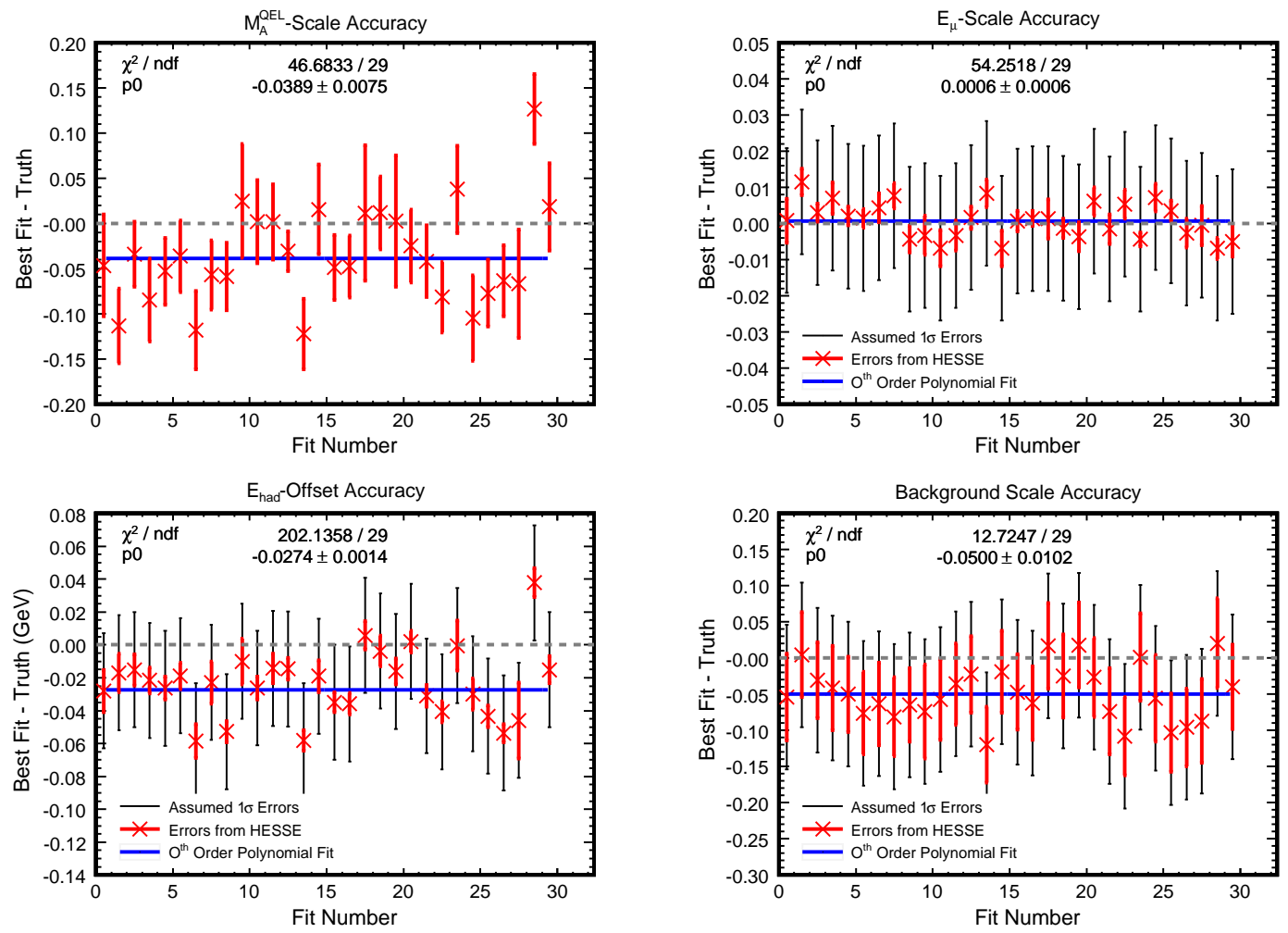

Figure 87: Best fit minus truth values for 30 shape and normalisation fits to the $Q_{Q E L}^{2}$ and $E_{\mathrm{v}}^{Q E L}$ distributions in mock data. The $x$-axes index the fits and the $y$-axes show the best-fit minus truth parameter values for the $M_{A}^{Q E L}$-scale (top left), $E_{\mu}$-scale (top right), $E_{\text {had }}$-offset (bottom left) and background scale (bottom right). The black error bars show the assumed $1 \sigma$ errors for each parameter whilst the red error bars show the symmetric errors reported by HESSE. The blue solid lines correspond to zeroth order polynominal fits to the points and use the errors reported by HESSE in calculating the fit $\chi^{2}$ which is displayed at the top of each figure. These $\chi^{2}$ values illustrate how well each distribution of best fit minus truth points corresponds to a constant value.

could potentially have errors similar to or smaller than all of the previous measurements of $M_{A}^{Q E L}$.

The small value of the RMS also shows that the fit is working well and, modulo the bias caused by the statistical differences between the mock data and MC fitted distributions, can correctly extract randomly generated $M_{A}^{Q E L}$ values with randomly generated systematic parameters applied to the mock data. These conclusions obviously assume that the $\mathrm{MC}$ is perfectly modelling the mock data, apart from the exact parameters that are included in the fit, and it is unlikely 

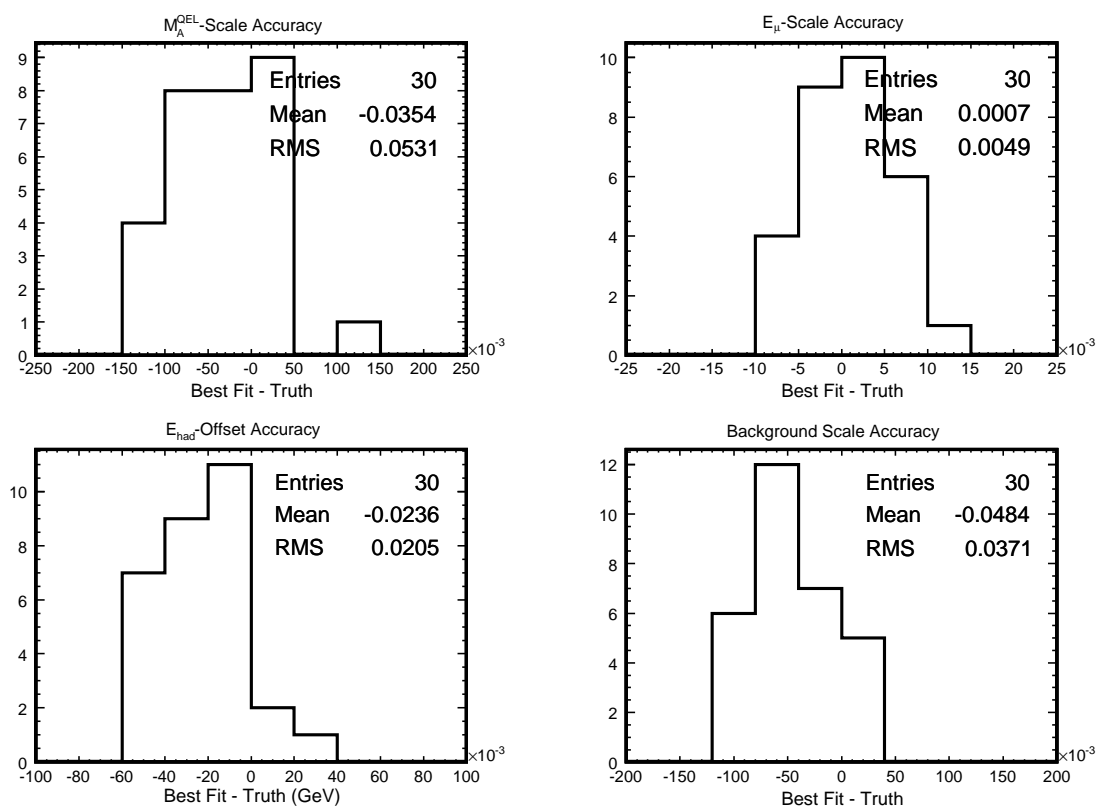

Figure 88: Projections of the 30 best fit minus truth parameter values from figure 87 along with the means and RMS values of these distributions.

that this is the case with the real data.

In addition to these 30 configuration II fits a further 30 fits were run with a modified fit configuration II. For fits to the data the low $Q_{Q E L}^{2}$ region may have to be excluded to obtain a reasonable fit and so this second set of 30 fits used the same configuration choices as defined by fit II but only fitted the $Q_{Q E L}^{2}$ distribution above $0.2 \mathrm{GeV}^{2}$ (these low $Q_{Q E L}^{2}$ events were used to fill the $E_{\mathrm{v}}^{Q E L}$ distribution). Table 10 reports the RMS values of the best fit minus truth parameter values from each of these two sets of 30 fits.

Table 10 shows that the sensitivity to $M_{A}^{Q E L}$ is degraded when only considering events with $Q_{Q E L}^{2}>0.2 \mathrm{GeV}^{2}$ in the fitted $Q_{Q E L}^{2}$ distribution. This is to be expected given that changes to $M_{A}^{Q E L}$ exhibit the largest $Q_{Q E L}^{2}$ distribution shape difference at low $Q_{Q E L}^{2}$. The systematic parameter sensitivites are quite similar for the two types of fits. 


\begin{tabular}{|c|c|c|c|c|}
\hline $\begin{array}{c}\text { Fit } \\
\text { Configuration }\end{array}$ & $\begin{array}{c}M_{A}^{Q E L} \text {-Scale } \\
\text { RMS }\end{array}$ & $\begin{array}{c}E_{\mu} \text {-Scale } \\
\text { RMS }\end{array}$ & $\begin{array}{c}E_{\text {had }} \text {-Offset } \\
\text { RMS }(\mathrm{GeV})\end{array}$ & $\begin{array}{c}\text { Background } \\
\text { Scale RMS }\end{array}$ \\
\hline \hline II & 0.0531 & 0.0049 & 0.0205 & 0.0371 \\
\hline II, no low $Q_{Q E L}^{2}$ & 0.0695 & 0.0048 & 0.0202 & 0.0377 \\
\hline
\end{tabular}

Table 10: RMS values for the distributions of best fit minus truth parameter values from 30 fits with configuration II and 30 fits with configuration II modified such that $Q_{Q E L}^{2}<0.2 \mathrm{GeV}^{2}$ events are not considered in the fitted $Q_{Q E L}^{2}$ distributions.

In addition to running many fits with configuration II large numbers of fits were run with configuration I. In these fits an attempt was made to remove some of the apparent bias caused by statistical differences between the mock data and MC samples. For each fit the mock data $Q_{Q E L}^{2}$ distribution was randomised by fluctuating the total number of events in the fitted distribution according to a Poisson distribution (centred at this original total number of events) and then filling a new mock data $Q_{Q E L}^{2}$ distribution by randomly sampling from the original distribution a number of times equal to the Poisson fluctuated total number of events. Unfortunately there is no obvious similar procedure that can be applied to the MC sample (which is filled for each iteration of a particular fit according to the current set of fit parameter values), which remains the same for each fit, and so an apparent fit bias will remain.

Figures 89 and 90 show the best fit minus truth parameter values for configuration I fits to the 100 randomised mock data samples and their projections. As with configuration II, another 100 fits were run that used a modified fit configuration I with the $Q_{Q E L}^{2}<0.2 \mathrm{GeV}^{2}$ events ignored in the fit. Table 11 shows the RMS values of the best fit minus truth parameter value projections for these two sets of 100 fits.

Table 11 shows similar trends as table 10 in that the removal of the low $Q_{Q E L}^{2}$ events degrades the sensitivity of the fit to $M_{A}^{Q E L}$ whereas the sensitivities to the systematic parameters are roughly equivalent between the two fit configurations. Comparing tables 10 and 11 is difficult given the different numbers of fit parameters and the different fit parameter correlations but both fits I and II seem to perform well, both with and without the low $Q_{Q E L}^{2}$ events. It should also be noted that the increase in the RMS values for the muon energy scale when moving 



Figure 89: Best fit minus truth values for 100 shape-only fits to the $Q_{Q E L}^{2}$ distribution in mock data. The $x$-axes index the fits and the y-axes show the best-fit minus truth parameter values for the $M_{A}^{Q E L}$-scale (top left), $E_{\mu}$-scale (top right), $E_{\text {had }}$-offset (bottom left) and background scale (bottom right). The black error bars show the assumed $1 \sigma$ errors for each parameter whilst the red error bars show the symmetric errors reported by HESSE. The blue solid lines correspond to zeroth order polynominal fits to the points and use the errors reported by HESSE in calculating the fit $\chi^{2}$ which is displayed at the top of each figure. These $\chi^{2}$ values illustrate how well each distribution of best fit minus truth points corresponds to a constant value.

\begin{tabular}{|c|c|c|c|c|}
\hline $\begin{array}{c}\text { Fit } \\
\text { Configuration }\end{array}$ & $\begin{array}{c}M_{A}^{Q E L} \text {-Scale } \\
\text { RMS }\end{array}$ & $\begin{array}{c}E_{\mu} \text {-Scale } \\
\text { RMS }\end{array}$ & $\begin{array}{c}E_{\text {had }} \text {-Offset } \\
\text { RMS }(\mathrm{GeV})\end{array}$ & $\begin{array}{c}\text { Background } \\
\text { Scale RMS }\end{array}$ \\
\hline \hline I & 0.0529 & 0.0067 & 0.0150 & 0.0291 \\
\hline I, no low $Q_{Q E L}^{2}$ & 0.0573 & 0.0066 & 0.0160 & 0.0272 \\
\hline
\end{tabular}

Table 11: RMS values for the distributions of best fit minus truth parameter values from 100 fits with configuration I and 100 fits with configuration I modified such that $Q_{Q E L}^{2}<0.2 \mathrm{GeV}^{2}$ events are not considered in the fitted $Q_{Q E L}^{2}$ distributions. 

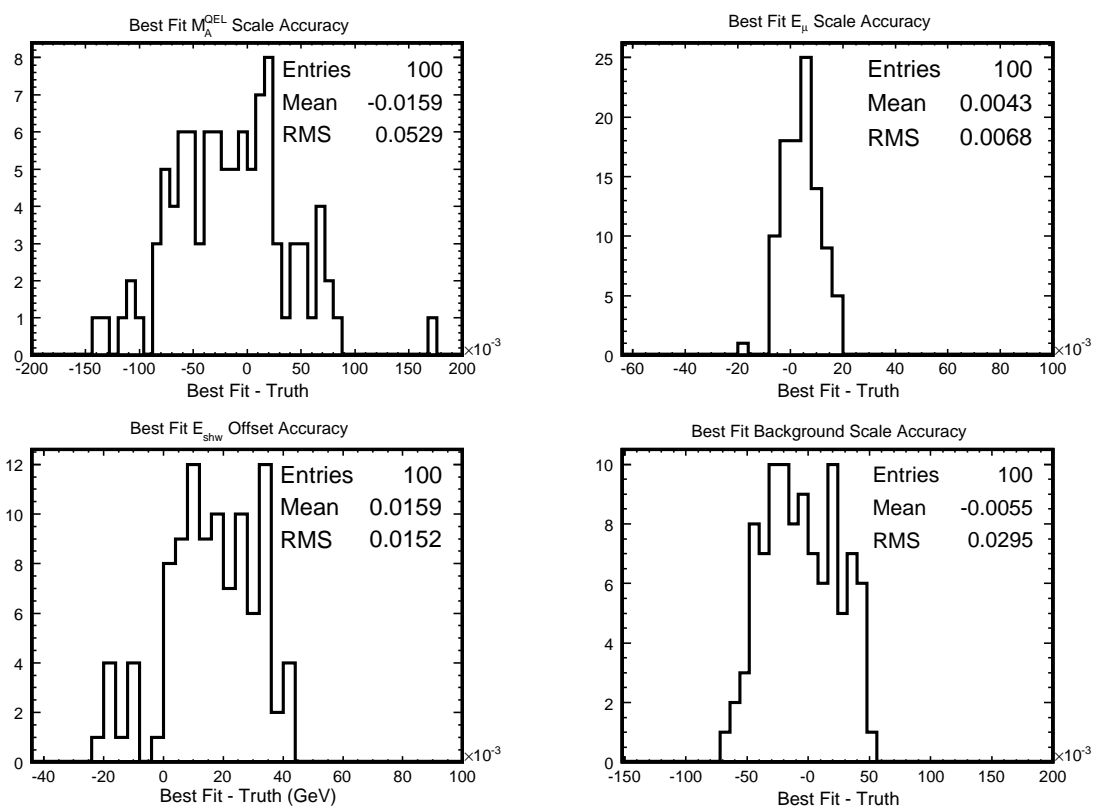

Figure 90: Projections of the 100 best fit minus truth parameter values from figure 89 along with the means and RMS values of these distributions. As before a bias is visible in the distribution means which could not be removed by fluctuating the mock data $Q_{Q E L}^{2}$ distributions.

from fit configuration II to configuration I is likely due to the increased correlation between the $M_{A}^{Q E L}$-scale and $E_{\mu}$-scale when only fitting for the shape of the $Q_{Q E L}^{2}$ distribution.

This chapter has presented the fit procedure for extracting $M_{A}^{Q E L}$ from a QEL-like sample of data in the ND. The main identified sources of systematic uncertainty are included directly in the fit and the resulting discontinuities in the fit $\chi^{2}$ surface are smoothed-out using an eventby-event smearing of MC kinematic variables. Example fit results have been shown in the two headline fit configurations; shape-only fit to $Q_{Q E L}^{2}$ and shape and normalisation fit to both $Q_{Q E L}^{2}$ and $E_{\mathrm{v}}^{Q E L}$. The fit has been studied using a mock data sample and the results of these studies show that the fit is robust to changes in it's internal mechanics and can correctly extract randomly chosen input mock data fit parameter values. The fit sensitivities are encouraging but do assume that the MC perfectly models the mock data and this assumption is not likely to be correct in the case of the real data. The next chapter will present the results of the $M_{A}^{Q E L}$ extraction from the real MINOS ND data. 


\section{$8 M_{A}^{Q E L}$ Measurement with ND Data}

This chapter will present results from a number of fits to the ND data. Two headline fit configurations are used; a shape-only fit to $Q_{Q E L}^{2}$ and a shape and normalisation fit to both $Q_{Q E L}^{2}$ and $E_{\mathrm{v}}^{Q E L}$. These fit configurations are very similar to those used for the mock data studies but do have a few differences that will be described in the following section. The results for the headline fits (and for a number of fits with small changes to the configurations) will be discussed in detail and finally some potential improvements for the fitting method will be suggested.

\subsection{Headline Fits to the Data}

The main differences between the headline fits to the mock data and these headline fits to the data correspond to the removal of exiting muon events (where the muon momentum was measured by curvature in the magnetic field) and to the way flux information is used in the fits. The following options are common to both the two headline data fit configurations:

- Stopping muons only: as was mentioned in the previous chapter the ND magnetic field has recently been re-calibrated and the results changed the momentum scale for muons that exit the detector by $\sim 6 \%$. However, the re-calibration resulted in only a small effect on the momentum scale for muons that stop in the detector and whose momenta are measured by range. As a result of the increased uncertainty on the energy of exiting muons only stopping muons are considered in the data and MC QEL-like samples used for the $M_{A}^{Q E L}$ fits. The removal of the exiting muon events does not result in a large loss in sample statistics, particularly in the energy spectrum peak where most of the muons do not have enough energy to exit the detector.

- Hadronic energy cut: the nominal hadronic energy cut of $<250 \mathrm{MeV}$ is used to select the QEL-like samples in data and MC.

- Minimised statistic: the $\chi_{M C S t a t s}^{2}$ statistic, as defined by equation 87 , is used in all fits to the data.

- Smearing of MC events: in all the fits to the data the $Q_{Q E L}^{2}, E_{\mathrm{v}}^{Q E L}$ and $E_{\text {had }}$ values for each MC event are smeared into Gaussians of widths $5 \mathrm{MeV}^{2}, 10 \mathrm{MeV}$ and $2.5 \mathrm{MeV}$ 
respectively.

- Systematic parameters: all the fits to the data will include the muon energy scale, hadronic energy offset and background scale systematic parameters with assumed $1 \sigma$ errors of $2 \%, 35 \mathrm{MeV}$ and $10 \%$ respectively.

- MINUIT routines: all the fits will call S IMPLEX, MIGRAD and then HESSE. Some of the fits will also include a final call to MINOS to provide the most accurate estimates of the errors on the fitted parameters.

- Sample exposures: the fitted data sample corresponds to a total exposure of $1.27 \mathrm{e} 20$ POT taken between May 2005 and March 2006 and using the L010z185i beam configuration. The MC sample used in the fits corresponds to a total exposure of 9.450e19 POT generated in the L010z185i beam configuration.

The two headline fits can then be further defined as follows:

- Data Fit I: shape-only fit to $Q_{Q E L}^{2}$. The $Q_{Q E L}^{2}$ distribution is binned in a similar way to mock data fit configuration I but the low $Q_{Q E L}^{2}$ region $\left(<0.2 \mathrm{GeV}^{2}\right)$, where the mismodelling of nuclear effects is likely, is not considered in the fit. The fitted distribution uses bins of width $0.1 \mathrm{GeV}^{2}$ from $0.2 \rightarrow 1.0 \mathrm{GeV}^{2}$ and with an overflow bin from 1.0 $\rightarrow 10.0 \mathrm{GeV}^{2}$. The flux factor systematic parameters are not used but instead the beam and hadron production tuning weights are applied to the MC so as to include the best knowledge of the NuMI flux.

- Data Fit II: shape and normalisation fit to $Q_{Q E L}^{2}$ and $E_{\mathrm{v}}^{Q E L}$. The fitted distributions use similar binning to that described for mock data fit configuration II but again the low $Q_{Q E L}^{2}$ region is removed from consideration for the fitted $Q_{Q E L}^{2}$ distribution. The $Q_{Q E L}^{2}$ distribution is binned as was described for data fit configuration I and the $E_{\mathrm{v}}^{Q E L}$ distibution binning is identical to that used for mock data fit configuration II. The low $Q_{Q E L}^{2}$ events are used when filling the neutrino energy distribution as the potential mismodelling of nuclear effects will not have a large effect on the reconstruction of $E_{\mathrm{v}}^{Q E L}$.

The beam and hadron production tuning weights are not applied to the MC but the seven flux factors are included in the fit as systematic parameters. They have assumed $1 \sigma$ 
errors of $8 \%$ for $E_{v}^{\text {true }} \leq 6 \mathrm{GeV}$ and $15 \%$ for $E_{v}^{\text {true }}>6 \mathrm{GeV}$ and the full matrix equation penalty term, as defined by equation 88 , is used to penalise the flux factors. The matrix of correlation coefficients defined by equation 89 is used when constructing this penalty term and as such only penalises deviations in adjacent flux factors.

\subsection{Results for Data Fit Confi guration I}

Table 12 summarises the results for this fit to the ND data. The best fit axial mass value is $\sim 1.39 \mathrm{GeV}$ compared to the nominal NEUGEN value of $0.99 \mathrm{GeV}$. The $\chi^{2}$ value is reduced from 135.965 with the nominal MC to 8.425 at the best fit and the fit contains eight degrees of freedom (corresponding to the nine bins of the fitted distribution minus the single free parameter). Figure 91 shows the fitted $Q_{Q E L}^{2}$ distributions along with the ratios of the data to the nominal and best fit $\mathrm{MC}$ and it can be seen that the best fit parameter values have flattened the ratio of data to $\mathrm{MC}$ as a function of $Q_{Q E L}^{2}$.

\begin{tabular}{|c|c|c|c|c|}
\hline & $\begin{array}{c}M_{A}^{Q E L}(\mathrm{GeV}) \\
\text { (Nominal: } 0.99 \mathrm{GeV})\end{array}$ & $E_{\mu}$-Scale & $\begin{array}{c}E_{\text {had }} \text {-Offset } \\
(\mathrm{GeV})\end{array}$ & $\begin{array}{c}\text { Background } \\
\text { Scale }\end{array}$ \\
\hline \hline Best Fit & 1.38616 & 0.97891 & -0.0323044 & 0.97938 \\
\hline HESSE Error & 0.04387 & 0.00299 & 0.0081325 & 0.07787 \\
\hline +ve MINOS Error & 0.07251 & 0.00331 & 0.0223089 & 0.07915 \\
\hline -ve MINOS Error & 0.05044 & 0.00315 & 0.0111125 & 0.07797 \\
\hline
\end{tabular}

Table 12: Best fit parameter values for a fit to the data with configuration I. The table shows the best fit values, the symmetric errors reported by HESSE and the asymmetric errors reported by MINOS. The correlation coefficients between the various fit parameters can be found in appendix $B$.

Table 12 shows that only the muon energy scale systematic parameter has been pulled by more than it's assumed $1 \sigma$ error (and only by $1.05 \sigma$ ). It is also interesting to note the differences between the parameter errors reported by HESSE and MINOS with the latter providing larger asymmetric errors, in particular for the axial-vector mass and the hadronic energy offset. This asymmetry reflects the non-linearity of the fitted 'problem' and is further demonstrated by figures 92 and 93 which show the two-dimensional fit contours between the various fit parameters (again using the approximation of equation 82). 



Figure 91: $Q_{Q E L}^{2}$ distributions for a fit to the data with configuration I. The left-hand figures show the full fitted $Q_{Q E L}^{2}$ distributions for the data, nominal unsmeared $M C$, nominal smeared $M C$ and best fit $M C$ along with ratios of the data to each MC set. The right-hand figures show the same distributions but zoomed such that the overflow bin is not visible.

Figure 92 shows that, modulo the elliptical approximation and uncertainty in the HESSE parameter correlation coefficients, all the systematic parameters are more highly correlated (or anti-correlated) with $M_{\AA}^{Q E L}$ than was seen in the example mock data fit with mock data fit configuration I. This is due to the removal of the low $Q_{Q E L}^{2}$ region from the fit where changes to $M_{A}^{Q E L}$ have the largest effect on the shape of the distribution. In particular the axial-vector mass and the muon energy scale are tightly anti-correlated and the contours illustrate that $\pm 1 \%$ changes to the muon energy scale could be accomodated by $\sim \pm 15 \%$ changes to the value of the $M_{A}^{Q E L}$-scale parameter. Figure 93 shows that there are no strong correlations between the three systematic parameters implying that, in the situation described above, a large change in 

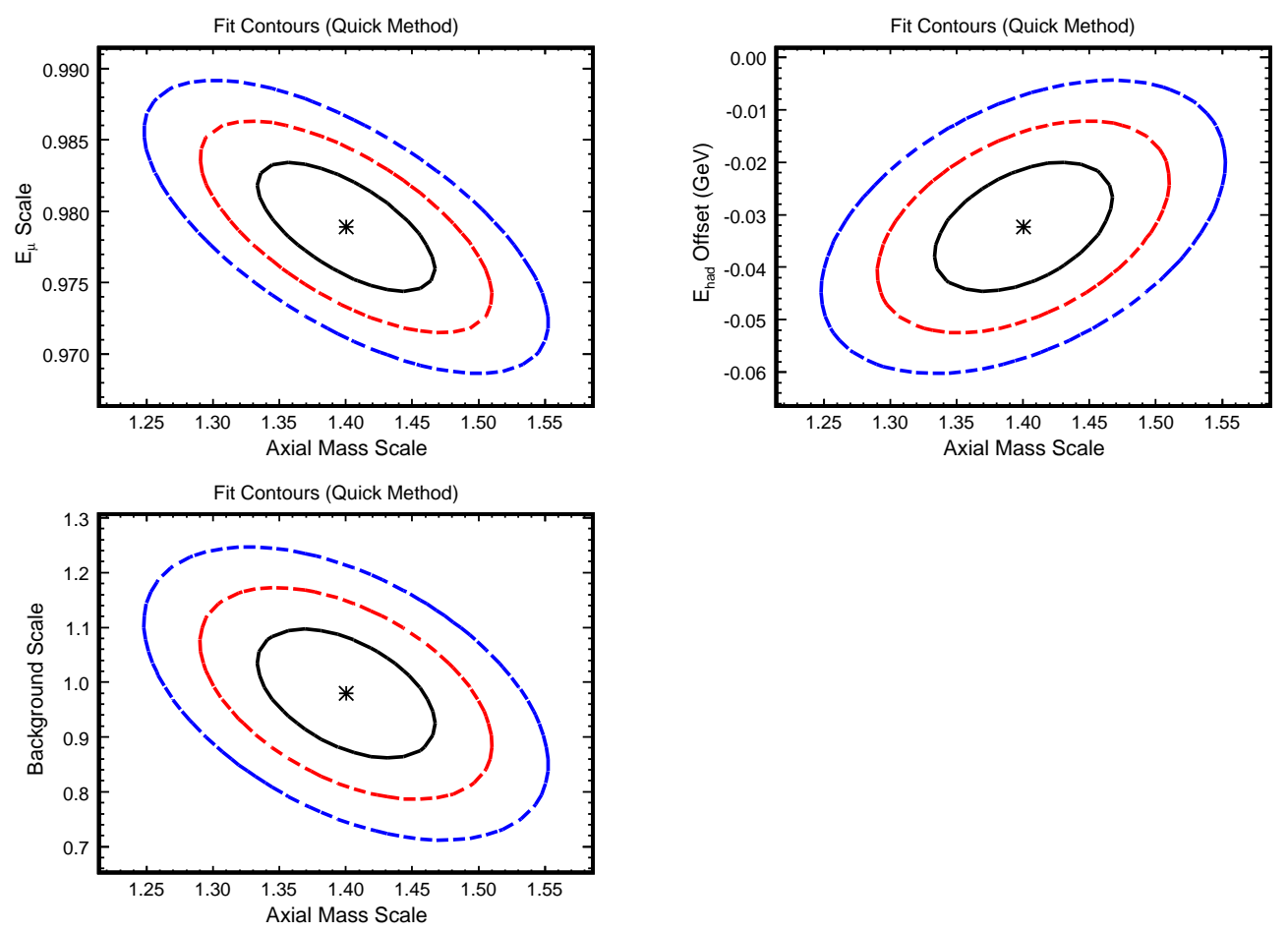

Figure 92: Two-dimensional fit contours for the $M_{A}^{Q E L}$-scale versus each of the three systematic parameters. These contours are made using an elliptical approximation and also use the parameter correlations reported by HESSE which should not be considered accurate to more than $\sim 10-20 \%$. The black point shows the best fit values and the black, red and blue ellipses show the $1 \sigma, 2 \sigma$ and $3 \sigma$ contours respectively.

the $M_{A}^{Q E L}$-scale could be accomodated by movement in a single systematic parameter, most likely the muon energy scale, without there being much of a pull on the remaining systematic parameters.

Figure 94 shows the POT-normalised comparisons of the data to the nominal and best fit MC, with a finer binning than was used in the fit itself, for the $Q_{Q E L}^{2}$ distributions of QEL-like events and also for the $Q^{2}$ distributions of CC-like events. It is not really valid to apply the best fit parameters to the entire CC-like distribution but this figure does suggest the kind of changes that would be induced in the CC-like sample. Figure 94 shows that even though the fit only considered the shape of the $Q_{Q E L}^{2}$ distribution it can do a good job in 'filling-in' the MC deficit. It also shows that in the excluded low $Q_{Q E L}^{2}$ region there is a definite disagreement between the 

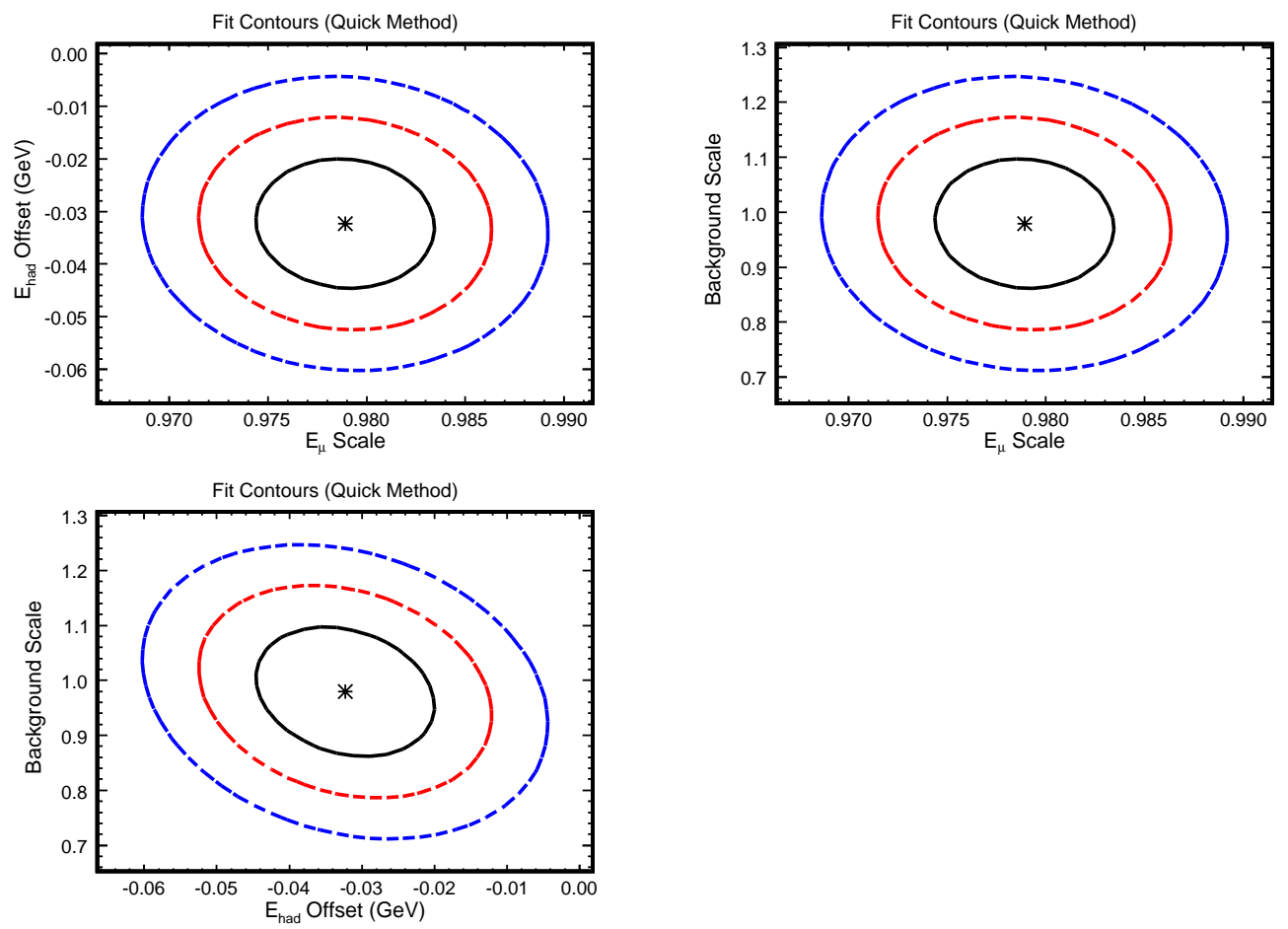

Figure 93: Two-dimensional fit contours for permutations of the three systematic parameters.

data and MC and this is attributed to incorrectly modelled nuclear effects. Similar low $Q_{Q E L}^{2}$ differences between QEL-like data and MC have been seen at previous experiments such as MiniBooNE [38].

Figure 95 shows the POT-normalised comparisons of the data to the nominal and best fit MC for the $E_{\mathrm{v}}^{Q E L}$ distributions of QEL-like events and the $E_{\mathrm{v}}$ distributions of CC-like events. The fit only used the $Q_{Q E L}^{2}$ distribution but there is a much improved agreement in the $E_{\mathrm{v}}^{Q E L}$ distribution for QEL-like events when using the best fit MC. Again it is not entirly valid to apply the best fit parameters when comparing the full CC-like sample but figure 95 shows that the best fit $\mathrm{MC}$ is in better agreement with the data and that, in particular, both the large deficit and shape difference of the nominal MC (relative to the data) in the peak of the energy spectrum have been mitigated. Figure 96 shows the two-dimensional ratios of the data to the nominal and best fit $\mathrm{MC}$ as a function of the reconstructed $Q_{Q E L}^{2}$ and $E_{\mathrm{v}}^{Q E L}$ and it can be seen that the best fit parameter values move the majority of these two-dimensional ratio bins close to unity. 

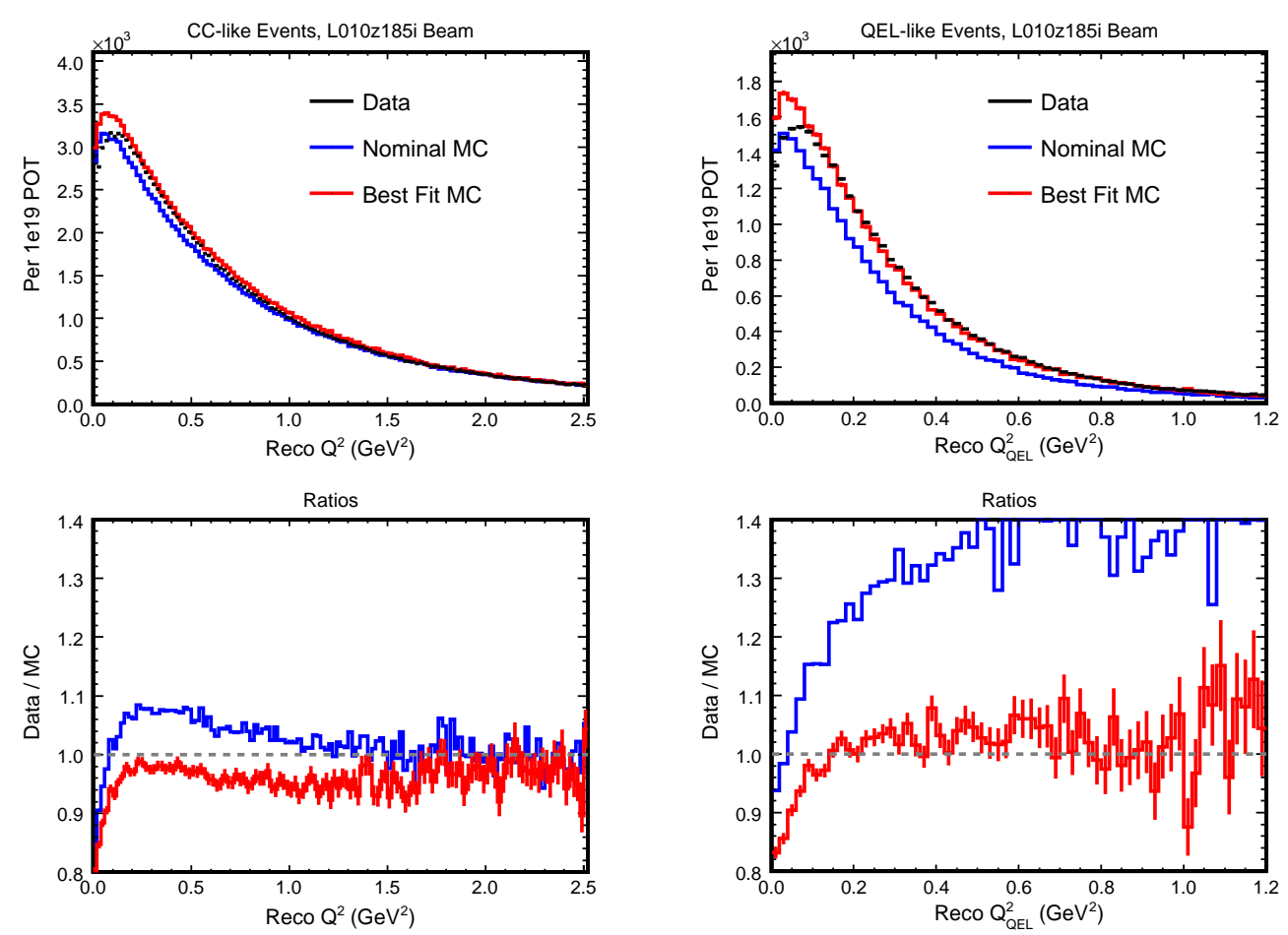

Figure 94: Comparisons of data, nominal and best fit $M C$ for $Q^{2}$ for all CC-like events (left-hand figures) and $Q_{Q E L}^{2}$ for $Q E L$-like events (right-hand figures). The shape-only fit both flattens the $Q_{Q E L}^{2}$ distribution, as should be expected, but also manages to provide an improved agreement in the normalisation between data and MC.

A number of fits were also run with small modifications to data fit configuration $I$ in order to check for consistency between the results; the data was fitted without the application of the beam and hadron production tuning weights to the $\mathrm{MC}$, different hadronic energy cuts were used to define the QEL-like sample and the data was fitted in a number of independant slices of reconstructed QEL-assumed neutrino energy. 

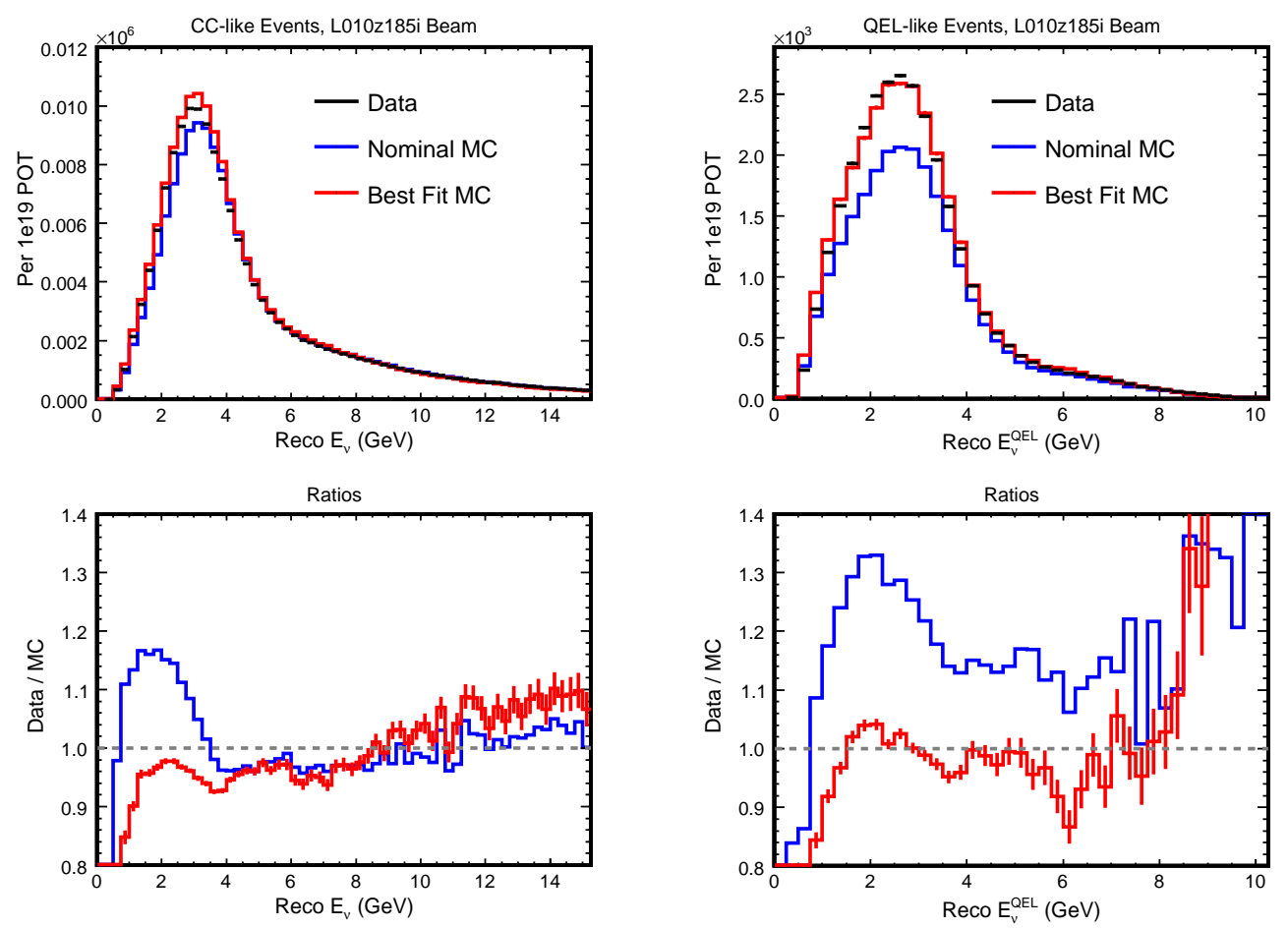

Figure 95: Comparisons of data, nominal and best fit MC for $E_{\mathrm{v}}$ for all CC-like events (left-hand figures) and $E_{\mathrm{v}}^{Q E L}$ for $Q E L$-like events (right-hand figures). The shape-only fit to the $Q_{Q E L}^{2}$ distribution results in an improved agreement between the data and MC neutrino energy spectra in both cases.
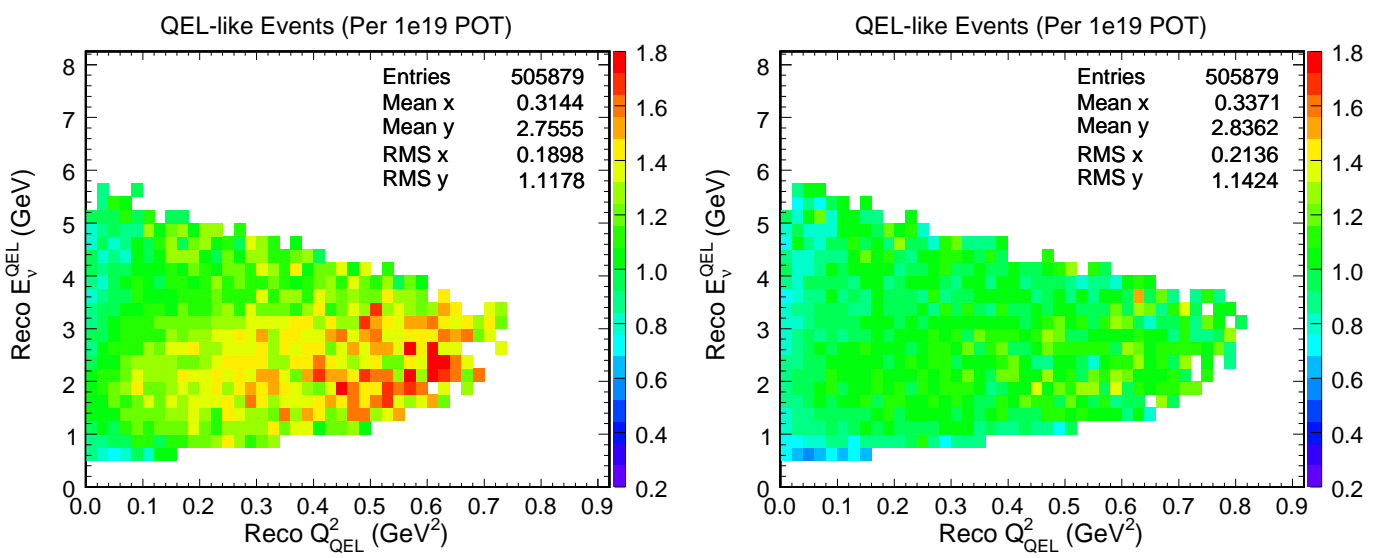

Figure 96: Two-dimensional ratios of the data to the nominal MC (left-hand figure) and to the best fit $M C$ (right-hand figure). For presentational purposes the bins in the ratios are only filled if the number of events in that bin, for both data and $M C$, is above ten. Apart from at low $Q_{Q E L}^{2}$ where nuclear effect mis-modelling is likely, the fit moves the majority of these ratio bins close to unity. 
Table 13 shows the headline fit I results from table 12 along with the results from an identical fit except that the beam and hadron production weights were not applied to the MC. The $Q_{Q E L}^{2}$ distribution is not very sensitive to the underlying neutrino flux and so the absense of the reweighting should not have a large impact on the results of the fit. Table 13 confirms that this is the case with all the fit parameters consistent within their MINOS errors between the two fits.

\begin{tabular}{|c|c|c|c|c|c|}
\hline $\begin{array}{c}\text { Fit } \\
\text { Configuration }\end{array}$ & $\begin{array}{c}M_{A}^{Q E L} \\
(\mathrm{GeV})\end{array}$ & $E_{\mu}$-Scale & $\begin{array}{c}E_{\text {had }} \text {-Offset } \\
(\mathrm{GeV})\end{array}$ & Bg-Scale & $\begin{array}{c}\left(\chi_{\text {Nom }}^{2}\right) \\
\chi_{B F}^{2}\end{array}$ \\
\hline \hline I & $\mathbf{1 . 3 8 6 1 6}$ & $\mathbf{0 . 9 7 8 9 1}$ & $\mathbf{- 0 . 0 3 2 3 0 4}$ & $\mathbf{0 . 9 7 9 3 8}$ & $(135.965)$ \\
& 0.04387 & 0.00299 & 0.008133 & 0.07787 & 8.42545 \\
& 0.07251 & 0.00331 & 0.022309 & 0.07915 & \\
& 0.05044 & 0.00315 & 0.011113 & 0.07797 & \\
\hline I without beam & $\mathbf{1 . 4 6 3 5 3}$ & $\mathbf{0 . 9 7 9 7 8}$ & $\mathbf{- 0 . 0 0 9 7 5 9}$ & $\mathbf{0 . 9 7 8 2 0}$ & $(170.096)$ \\
and hadron & 0.05307 & 0.00287 & 0.012996 & 0.08370 & 9.45951 \\
weights applied & 0.06378 & 0.00304 & 0.013776 & 0.09367 & \\
to the MC & 0.07581 & 0.00293 & 0.023603 & 0.09828 & \\
\hline
\end{tabular}

Table 13: Results for data fit configuration I with and without the beam and hadron production tuning weights applied to the MC. The bold numbers show the best fit values whilst the row of numbers directly beneath show the symmetric errors reported by HESSE. The final two rows of numbers for each fit show the MINOS positive (3rd row) and negative (4th row) errors. The last column in the table shows the inital $\chi^{2}$ value between the data and nominal MC in brackets and the best fit $\chi^{2}$ value below this.

Table 14 shows the results from the fit to the data with configuration I along with fits that were performed with the same configuration but only using events from particular slices of reconstructed $E_{\mathrm{V}}^{Q E L}$. It can be seen that the fits in the slices $0.0 \rightarrow 2.0 \mathrm{GeV}$ and $3.0 \rightarrow 4.0 \mathrm{GeV}$ produced results that are very consistent with the original fit, to events from the full neutrino energy spectrum, but that the fit in the slice from $2.0 \rightarrow 3.0 \mathrm{GeV}$ has produced a very different set of best fit parameter values.

In this neutrino energy slice the best fit $M_{A}^{Q E L}$ is much lower than that from headline fit I, the muon energy scale is now pulled higher and the hadronic energy offset and background scale parameters are pulled by more than their assumed $1 \sigma$ errors. Figure 97 shows comparisons of the QEL-like data, nominal MC and best fit MC (all normalised to POT) using these different 


\begin{tabular}{|c|c|c|c|c|c|}
\hline $\begin{array}{c}\text { Fit } \\
\text { Configuration }\end{array}$ & $\begin{array}{c}M_{A}^{Q E L} \\
(\mathrm{GeV})\end{array}$ & $E_{\mu}$-Scale & $\begin{array}{c}E_{\text {had }} \text {-Offset } \\
(\mathrm{GeV})\end{array}$ & Bg-Scale & $\begin{array}{c}\left(\chi_{\text {Nom }}^{2}\right) \\
\chi_{B F}^{2}\end{array}$ \\
\hline \hline I & $\mathbf{1 . 3 8 6 1 6}$ & $\mathbf{0 . 9 7 8 9 1}$ & $\mathbf{- 0 . 0 3 2 3 0 4}$ & $\mathbf{0 . 9 7 9 3 8}$ & $(135.965)$ \\
& 0.04387 & 0.00299 & 0.008133 & 0.07787 & 8.42545 \\
& 0.07251 & 0.00331 & 0.022309 & 0.07915 & \\
& 0.05044 & 0.00315 & 0.011113 & 0.07797 & \\
\hline I with $E_{\mathrm{v}}^{Q E L}$ & $\mathbf{1 . 3 8 1 5 2}$ & $\mathbf{0 . 9 8 6 7 2}$ & $\mathbf{- 0 . 0 0 0 0 9 2}$ & $\mathbf{0 . 9 8 4 2 9}$ & $(39.089)$ \\
$\in(0.0,2.0) \mathrm{GeV}$ & 0.07284 & 0.00393 & 0.005023 & 0.09705 & 13.69950 \\
& 0.07610 & 0.00381 & 0.007774 & 0.09761 & \\
\hline I with $E_{\mathrm{v}}^{Q E L}$ & 0.07210 & 0.00476 & 0.005619 & 0.09793 & \\
$\in[2.0,3.0) \mathrm{GeV}$ & $\mathbf{1 . 0 7 8 1 3}$ & $\mathbf{1 . 0 1 0 8 6}$ & $\mathbf{- 0 . 0 4 0 8 9 9}$ & $\mathbf{0 . 8 2 8 5 5}$ & $(55.735)$ \\
& 0.05728 & 0.00387 & 0.006220 & 0.08106 & 19.07930 \\
& 0.05655 & 0.00451 & 0.042261 & 0.08068 & \\
\hline I with $E_{\mathrm{v}}^{Q E L}$ & 0.05911 & 0.00445 & 0.047586 & 0.08242 & \\
$\in[3.0,4.0) \mathrm{GeV}$ & $\mathbf{1 . 4 0 2 3 1}$ & $\mathbf{0 . 9 8 2 4 2}$ & $\mathbf{- 0 . 0 3 3 8 0 8}$ & $\mathbf{0 . 9 9 5 0 1}$ & $(100.249)$ \\
& 0.04615 & 0.00606 & 0.007864 & 0.08752 & 6.38665 \\
& 0.05944 & 0.00734 & 0.033593 & 0.09148 & \\
& 0.09136 & 0.00569 & 0.024293 & 0.09113 & \\
\hline
\end{tabular}

Table 14: Results for data fit configuration I using events from the full $E_{\mathrm{v}}^{Q E L}$ distribution and in a number of independant slices of $E_{\mathrm{v}}^{Q E L}$. The table structure is identical to that described in the caption for table 13.

best fit parameter values. It can be seen that this shape-only fit has performed as it should with the ratio of data to best fit $\mathrm{MC}$ being flat in the fit region (above $Q_{Q E L}^{2}=0.2 \mathrm{GeV}^{2}$ ) but in this particular case the best fit results do not do such a good job filling-in the MC deficit.

When comparing these results to the results from the nominal configuration I fit to the data the best fit $M_{A}^{Q E L}$ and $E_{\mu}$-scale values are not consistent within their MINOS errors, however, these are not bad fits. Rather the difference reflects the strong anti-correlation between these two parameters in a shape-only fit to the $Q_{Q E L}^{2}$ distribution (see figure 92 or appendix B) with a reduction in $M_{A}^{Q E L}$ being countered by an increase in the muon energy scale. This shapeonly fit configuration is not very sensitive to the hadronic energy offset and background scale systematic parameters (which mostly affect the normalisation of the $Q_{Q E L}^{2}$ distribution) but they are (anti-)correlated with $M_{A}^{Q E L}$ and so large changes in their values should not be unexpected. 



Figure 97: POT-normalised data, nominal and best fit $M C Q_{Q E L}^{2}$ distributions, along with the ratio of the data to each MC set, using the results from a fit to the data with configuration I but only considering events with $E_{\mathrm{v}}^{Q E L} \in[2.0,3.0) \mathrm{GeV}$.

One possible explanation for the anomalous fit result, presented in table 14, is that the fit has converged upon a local minimum in it's four-dimensional internal $\chi^{2}$-space and that statistical differences between the data samples, coming from the different slices of neutrino energy considered, led MIGRAD into this local minimum instead of towards the region of parameter space suggested by the other fits in table 14 . The errors reported by MINOS can be fooled by such local minima if they are sufficiently far apart in parameter space and if the $\chi^{2}$ surface rises steeply between these minima.

In order to test this hypothesis an alternate 'grid search' fitting method was employed. In the grid search the hadronic energy offset and background scale fit parameters, which do not have a large effect on the shape of the $Q_{Q E L}^{2}$ distribution, were held constant at the best fit values returned by the fit in headline configuration I (as shown in table 12). The grid search then calculated the $\chi^{2}$ value between the MC and data using the shape of the $Q_{Q E L}^{2}$-distribution (using the same construction as the MINUIT fits) at a number of points in the two-dimensional parameter space of ( $M_{A}^{Q E L}$-scale, $E_{\mu}$-scale) and recorded both the minimum $\chi^{2}$ and the parameter values to which this minimum corresponded. Figure 98 shows the $\chi^{2}$-spaces for grid searches performed using events from the full range of possible neutrino energies (as used in headline fit I) and using events with neutrino energies between 2.0 and $3.0 \mathrm{GeV}$ (with which the MINUIT fit produced the anomalous fit result). 



Figure 98: $\chi^{2}$-surfaces for grid searches using events with all neutrino energies (left-hand figure) and those in a slice from $2.0 \rightarrow 3.0 \mathrm{GeV}$ (right-hand figure). The statistical differences between the $Q_{Q E L}^{2}$ distributions for the two data samples with which these grid searches were performed result in a slight warping of the $\chi^{2}$-surface although the large anti-correlation between the two parameters is seen in both cases.

The grid search performed using events of all neutrino energies found a best fit point in excellent agreement with the results from the corresponding MINUIT fit with a best fit $M_{A}^{Q E L}$-scale of 1.40 (as compared to 1.4002 from MINUIT), a best fit $E_{\mu}$-scale of 0.979 (as compared to 0.97891 from MINUIT) and a best fit $\chi^{2}$ value of 8.427 (as compared to 8.425 from MINUIT). However, the grid search performed using events with neutrino energies between 2.0 and 3.0 $\mathrm{GeV}$ found a very different minimum to that found by the corresponding MINUIT fit. Figure 99 shows a zoomed version of the $\chi^{2}$-surface from the grid search to events in this neutrino energy slice and confirms the above hypothesis that there are both a local and a global minimum in the $\chi^{2}$-space of this fit.

In this case the MINUIT fit results correspond to the local minimum that can be seen in figure 99 whereas the grid search locates the global minimum with a best fit $M_{A}^{Q E L}$-scale of 1.35 and a best fit $E_{\mu}$-scale of 0.976 . These results are in good agreement with the MINUIT fit results from all but the original $2.0 \rightarrow 3.0 \mathrm{GeV}$ slice fit as shown in table 14 and the best fit $\chi^{2}$ for this energy slice is 12.421 (as compared to 19.079 as returned by the corresponding MINUIT fit). The MINUIT fit in the $2.0 \rightarrow 3.0 \mathrm{GeV}$ neutrino energy slice was repeated with MIGRAD starting at the best fit parameter values returned by headline fit I. In this case the fit converged upon the global minimum and table 15 shows a repeated version of the results from headline fit I along 
with these new results.

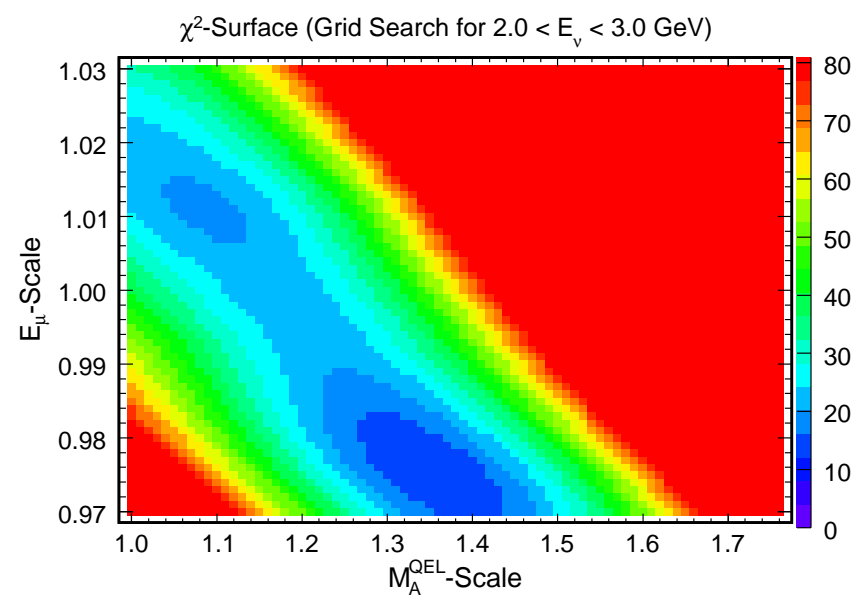

Figure 99: Zoom in on the $\chi^{2}$-surface from a grid search using events with neutrino energies in a slice from $2.0 \rightarrow 3.0 \mathrm{GeV}$. The corresponding MINUIT fit found the local minimum visible in the upper left of the figure whilst the grid search correctly located the global minimum in the $\chi^{2}$-space.

\begin{tabular}{|c|c|c|c|c|c|}
\hline $\begin{array}{c}\text { Fit } \\
\text { Configuration }\end{array}$ & $\begin{array}{c}M_{A}^{Q E L} \\
(\mathrm{GeV})\end{array}$ & $E_{\mu}$-Scale & $\begin{array}{c}E_{\text {had }} \text {-Offset } \\
(\mathrm{GeV})\end{array}$ & Bg-Scale & $\begin{array}{c}\left(\chi_{\text {Nom }}^{2}\right) \\
\chi_{B F}^{2}\end{array}$ \\
\hline \hline I & $\mathbf{1 . 3 8 6 1 6}$ & $\mathbf{0 . 9 7 8 9 1}$ & $\mathbf{- 0 . 0 3 2 3 0 4}$ & $\mathbf{0 . 9 7 9 3 8}$ & $(135.965)$ \\
& 0.04387 & 0.00299 & 0.008133 & 0.07787 & 8.42545 \\
& 0.07251 & 0.00331 & 0.022309 & 0.07915 & \\
& 0.05044 & 0.00315 & 0.011113 & 0.07797 & \\
\hline I with $E_{\mathrm{v}}^{Q E L}$ & $\mathbf{1 . 3 1 1 5 2}$ & $\mathbf{0 . 9 7 4 9 5}$ & $\mathbf{- 0 . 0 4 1 6 6 3}$ & $\mathbf{0 . 9 3 5 3 7}$ & $(22.1397)$ \\
$\in[2.0,3.0) \mathrm{GeV}$ & 0.05192 & 0.00331 & 0.009017 & 0.09512 & 11.16540 \\
& 0.05137 & 0.00327 & 0.025012 & 0.09564 & \\
& 0.15325 & 0.00778 & 0.043479 & 0.09602 & \\
\hline
\end{tabular}

Table 15: Results for data fit configuration I using events from the full $E_{\mathrm{v}}^{Q E L}$ distribution and for the repeated fit considering events with neutrino energies between 2.0 and $3.0 \mathrm{GeV}$.

The grid searches have shown that the best fit parameters corresponding to the true global minima in each of the fits presented in table 15 agree within their errors but also highlight a possible problem with the use of MINUIT. In the future fits could be run that include an extra 
call to the MINUIT routine IMPROVE which helps to guard against local minima by randomly stepping away from the calculated minimum in parameter space for some number of iterations and trying a quick convergence starting at each of these randomly chosen points. Another possible alternative for the future, applicable to type I fits, would be a hybrid method that used a course grid search in $M_{A}^{Q E L}$ and the muon energy scale combined with calls to MINUIT. In such a fit a minimum would be found at each grid point by minimising with respect to the hadronic energy offset and background scale parameters and then the parameters from the grid bin with the lowest $\chi^{2}$ would be fed to MIGRAD, HESSE and then MINOS to ensure that these MINUIT routines were called in the vicinity of the global minimum.

A number of fits were also performed with configuration I but changing the value of the hadronic energy cut used to define the QEL-like samples of data and MC. Table 16 shows the results of the headline fit along with fits where the hadronic energy cut was set to $225 \mathrm{MeV}$ and $275 \mathrm{MeV}$ and it can be seen that both these fits return parameter values that are consistent, within their errors, with the headline fit.

\begin{tabular}{|c|c|c|c|c|c|}
\hline $\begin{array}{c}\text { Fit } \\
\text { Configuration }\end{array}$ & $\begin{array}{c}M_{A}^{Q E L} \\
(\mathrm{GeV})\end{array}$ & $E_{\mu}$-Scale & $\begin{array}{c}E_{\text {had }} \text {-Offset } \\
(\mathrm{GeV})\end{array}$ & Bg-Scale & $\begin{array}{c}\left(\chi_{\text {Nom }}^{2}\right) \\
\chi_{B F}^{2}\end{array}$ \\
\hline \hline I & $\mathbf{1 . 3 8 6 1 6}$ & $\mathbf{0 . 9 7 8 9 1}$ & $\mathbf{- 0 . 0 3 2 3 0 4}$ & $\mathbf{0 . 9 7 9 3 8}$ & $(135.965)$ \\
& 0.04387 & 0.00299 & 0.008133 & 0.07787 & 8.42545 \\
& 0.07251 & 0.00331 & 0.022309 & 0.07915 & \\
& 0.05044 & 0.00315 & 0.011113 & 0.07797 & \\
\hline I with $E_{\text {had }}$ & $\mathbf{1 . 4 2 1 7 9}$ & $\mathbf{0 . 9 7 9 3 1}$ & $\mathbf{- 0 . 0 2 7 2 6 6}$ & $\mathbf{0 . 9 6 4 3 5}$ & $(143.804)$ \\
cut at & 0.05720 & 0.00465 & 0.010509 & 0.08055 & 8.67243 \\
$225 \mathrm{MeV}$ & 0.05152 & 0.00318 & 0.008047 & 0.09082 & \\
& 0.07230 & 0.00340 & 0.020261 & 0.08015 & \\
\hline I with $E_{\text {had }}$ & $\mathbf{1 . 3 2 6 1 1}$ & $\mathbf{0 . 9 7 9 2 8}$ & $\mathbf{- 0 . 0 4 8 7 5 8}$ & $\mathbf{1 . 0 0 4 7 6}$ & $(150.031)$ \\
cut at & 0.04241 & 0.00213 & 0.009684 & 0.08523 & 11.32380 \\
$275 \mathrm{MeV}$ & 0.15909 & 0.00346 & 0.044459 & 0.08367 & \\
& 0.05796 & 0.00557 & 0.013047 & 0.15446 & \\
\hline
\end{tabular}

Table 16: Results for data fit configuration I using QEL-like samples defined with the nominal hadronic energy cut at $250 \mathrm{MeV}$ and with two alternate hadronic energy cuts at $225 \mathrm{MeV}$ and $275 \mathrm{MeV}$. The table structure is identical to that described in the caption for table 13. 
Table 16 reveals some interesting asymmetric MINOS errors reported for the fit with the hadronic energy cut at $275 \mathrm{MeV}$, in particular the larger positive error on $M_{A}^{Q E L}$ and the larger negative error on the background scale parameter. It has already been seen that in this fit configuration there is some correlation between these two parameters and the larger MINOS errors suggest that, for this fit, $M_{A}^{Q E L}$ could be increased and the background scale decreased (bringing the fitted parameter values more in line with the other two sets of fit results presented in table 16) without the value of the $\chi^{2}$ changing significantly.

In summary, all of the fits performed using configuration type I agree that the value for $M_{A}^{Q E L}$ should be increased dramatically from it's default NEUGEN value of $0.99 \mathrm{GeV}$. A number of fits using different permutations of the configuration options have shown that the correlations between the fit parameters, when combined with statistical differences resulting from these changed options, can result in movement of the best fit parameter values. However, these movements are either within or just outside the quoted MINOS errors returned by MINUIT.

As such, the errors on the best fit $M_{A}^{Q E L}$ for these shape-only fits to $Q_{Q E L}^{2}$ are presented in two parts; the asymmetric errors given by the MINOS routine and additional asymmetric errors coming from the largest change in the fitted central value stemming from the different permutations of the fit configuration. The largest upward shift comes from the fit without the beam and hadron production weighting applied to the MC and the largest downward shift comes from the fit in the $2.0 \rightarrow 3.0 \mathrm{GeV}$ slice of neutrino energy. These two components are referred to in equation 92 as the statistical (MINOS errors) and systematic (change in central value under permutations) errors on the fitted $M_{A}^{Q E L}$. It should be noted that in this case the statistical error has a component due to the fitted systematic parameters (in particular the muon energy scale) which is not intrinsically statistical in nature and cannot be decoupled from the true statistical error in the fit and likewise the systematic error has a component that is statistical in nature due to the fact that these errors come from alternate fits to the data.

$$
M_{A}^{Q E L}=1.3866_{-0.050}^{+0.073}(\text { stat. })_{-0.075}^{+0.077} \text { (syst.) } \mathrm{GeV}
$$

The following section will now present results obtained using configuration type II. 


\subsection{Results for Data Fit Confi guration II}

Table 17 summarises the results when the data is fit using this configuration. The best fit $M_{A}^{Q E L}$ value is $\sim 1.13 \mathrm{GeV}$ and is again higher than the nominal NEUGEN value of $0.99 \mathrm{GeV}$. In this fit the $\chi^{2}$ value between the data and MC is reduced from 20227.6 with the nominal MC to 244.748 when the best fit parameters are applied to the MC and the fit contains twenty-one degrees of freedom; nine degrees from the bins of the $Q_{Q E L}^{2}$ distribution plus thirteen degrees from the bins of the $E_{\mathrm{v}}^{Q E L}$ distribution minus one degree from the single free parameter, $M_{A}^{Q E L}$. The reduced $\chi^{2}$ at the best fit point suggests that this is a bad fit and it will be demonstrated during the following discussion that this high value is a product of the crudeness of the flux factor systematic parameters. Due to computer processing time considerations MINOS errors were only calculated for the non-flux factor fit parameters.

For this set of results many of the fit parameters have been pulled much further than their assumed $1 \sigma$ errors. In particlar the flux in the peak has been increased and the hadronic energy offset made negative so as to 'fill-in' the large MC deficit in the energy spectrum peak whilst conversely the background scale has been reduced, presumably to compensate for some of this increase in the event numbers. The muon energy scale has not changed greatly, reflecting the fact that the position of the energy spectum peak is well modelled, even by the nominal MC, and the axial mass has been increased slightly to correct the shape of the $Q_{Q E L}^{2}$ distribution.

Figure 100 shows the fitted $Q_{Q E L}^{2}$ and $E_{\mathrm{v}}^{Q E L}$ distributions along with the ratios of the data to the best fit and nominal $\mathrm{MC}$ and shows that at the best fit the data to $\mathrm{MC}$ ratio is flat compared with the nominal case. The $E_{\mathrm{v}}^{Q E L}$ ratio for the data over best fit $\mathrm{MC}$ demonstrates the problem introduced by the flux factors which is that they scale large chunks of the true neutrino energy distribution and the smearing introduced by moving to a reconstructed quantity is not sufficient to remove the discrete nature of the different best fit flux factor values. As a result of their crude nature, the flux factors result in disagreements between the contents of the data and MC reconstructed neutrino energy bins which inflate the best fit $\chi^{2}$ value. The best fit $\chi^{2}$ value of 244.748 can be broken down into components coming from the $Q_{Q E L}^{2}$ distribution bin contents, the $E_{\mathrm{v}}^{Q E L}$ distibution bin contents, the non-flux factor systematic parameter penalty term and 


\begin{tabular}{|c|c|c|c|c|}
\hline \multirow{2}{*}{$\begin{array}{c}\text { Fit } \\
\text { Configuration }\end{array}$} & \multicolumn{4}{|c|}{ II } \\
\hline & $\begin{array}{l}\text { Best Fit } \\
\text { Value }\end{array}$ & $\begin{array}{l}\text { HESSE } \\
\text { Error }\end{array}$ & $\begin{array}{c}\text { +ve MINOS } \\
\text { Error }\end{array}$ & $\begin{array}{c}\text {-ve MINOS } \\
\text { Error }\end{array}$ \\
\hline$M_{A}^{Q E L}(\mathrm{GeV})$ & 1.13026 & 0.01964 & 0.02018 & 0.02051 \\
\hline$E_{\mu}$-Scale & 1.01787 & 0.00119 & 0.00171 & 0.00150 \\
\hline$E_{\text {had }}$-Offset $(\mathrm{GeV})$ & -0.070314 & 0.003327 & 0.003466 & 0.003907 \\
\hline Background Scale & 0.74427 & 0.04457 & 0.04566 & 0.4457 \\
\hline 0-2 GeV Flux Scale & 1.20484 & 0.02931 & & \\
\hline 2-3 GeV Flux Scale & 1.31674 & 0.02262 & & \\
\hline 3-4 GeV Flux Scale & 1.01731 & 0.01444 & & \\
\hline 4-6 GeV Flux Scale & 0.97623 & 0.01585 & & \\
\hline 6-10 GeV Flux Scale & 1.35798 & 0.03124 & & \\
\hline$\overline{\chi_{\text {Nom }}^{2}}$ & \multicolumn{4}{|c|}{20227.6} \\
\hline$\chi_{B F}^{2}$ & \multicolumn{4}{|c|}{244.748} \\
\hline
\end{tabular}

Table 17: Best fit parameter values for a fit to the data with configuration II. The table shows the best fit values, the symmetric errors reported by HESSE and the asymmetric errors reported by MINOS. The MINOS errors were not calculated for the flux factors. The correlation coefficients between the various fit parameters can be found in appendix $B$.

the flux factor penalty term and in this case the contributions are $37.8,169.8,11.4$ and 25.8 respectively. The largest contribution comes from the $E_{\mathrm{v}}^{Q E L}$ bin contents.

Figure 101 shows more finely binned versions of the fitted distributions shown in figure 100 and further demonstrates both that the low $Q_{Q E L}^{2}$ regime is not well modelled by the MC and that the best fit flux factors introduce a 'sinusoidal' warping to the $\mathrm{MC} E_{\mathrm{v}}^{Q E L}$ distribution. Figure 102 shows the two-dimensional ratios of the data to the nominal and best fit MC and it can be seen that the best fit parameter values flatten large parts of this surface with the remaining deviations of the $\mathrm{MC}$ from the data located, as expected, at low $Q_{Q E L}^{2}$. 



Figure 100: $Q_{Q E L}^{2}$ (zoomed to exclude the overflow bin) and $E_{\mathrm{v}}^{Q E L}$ distributions for a fit to the data with configuration II. The top figures show the fitted distributions for the data, nominal unsmeared MC, nominal smeared MC and best fit MC whilst the bottom figures show the ratios of the data to each MC set. 

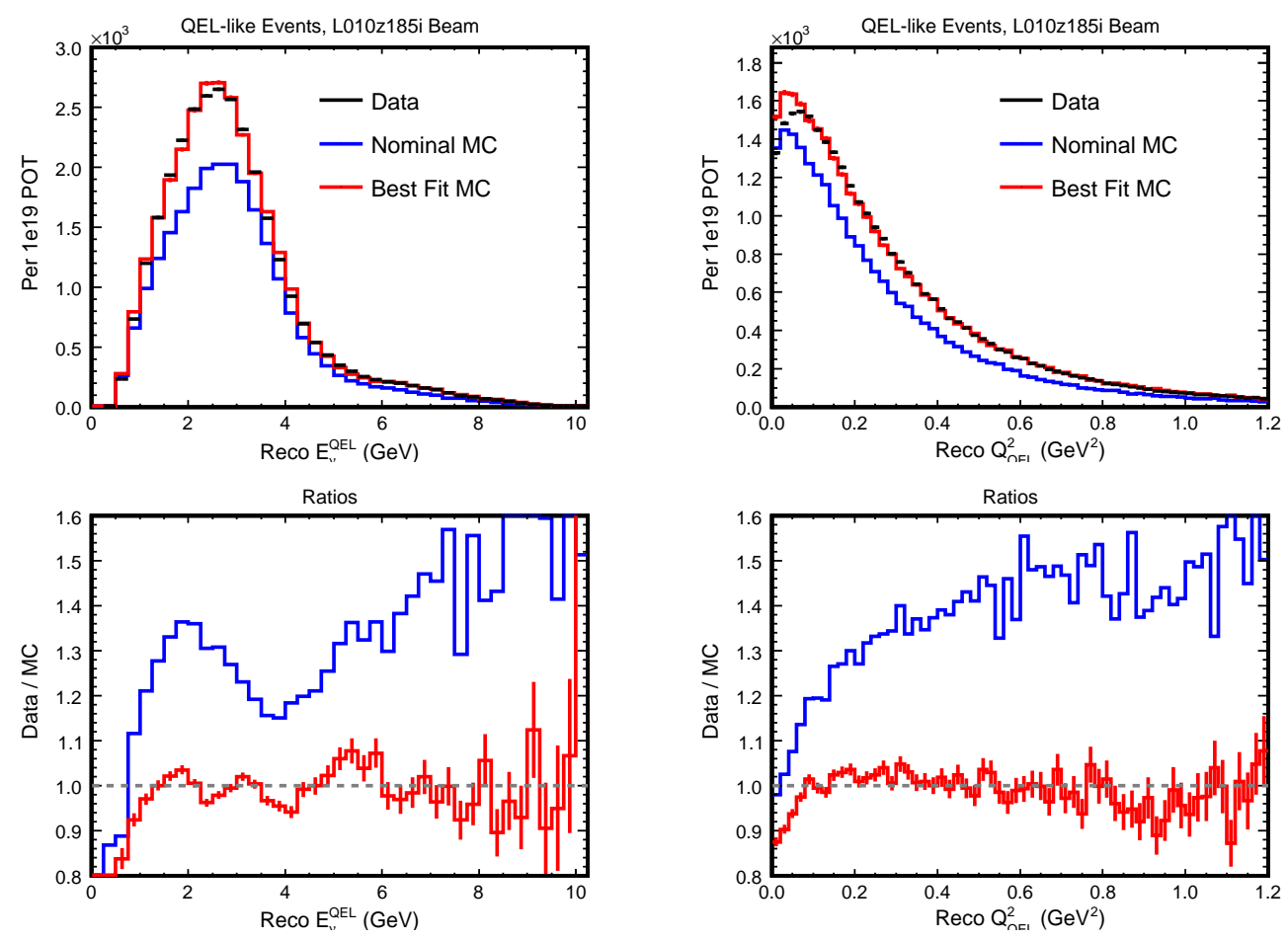

Figure 101: Comparisons of data, nominal and best fit MC as a function of $E_{\mathrm{v}}^{Q E L}$ (left-hand figures) and $Q_{Q E L}^{2}$ (right-hand figures). The results from headline fit II can be seen to be flattening the ratios as a function of both parameters but the crudeness of the flux factors is evident in the ratio of the data to best fit MC as a function of the recontructed neutrino energy.
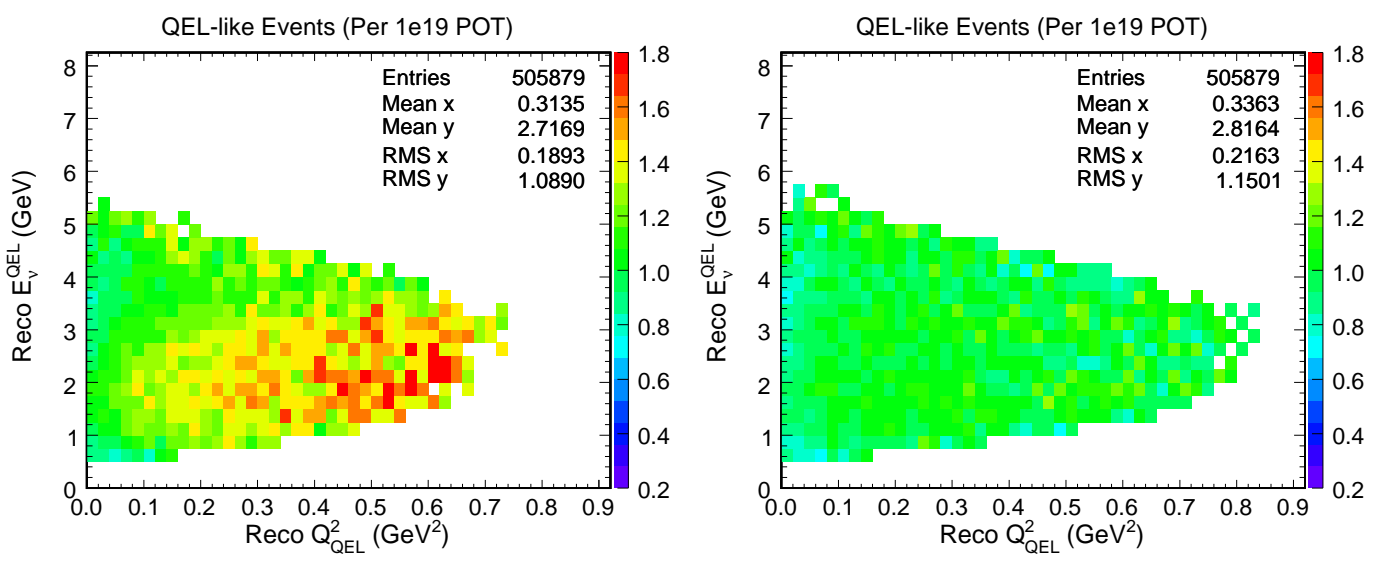

Figure 102: Two-dimensional ratios of the data to the nominal MC (left-hand figure) and to the best fit $M C$ (right-hand figure). The bins in the ratios are only filled if the number of events in that bin, for both data and $M C$, is above ten. Apart from at low $Q_{Q E L}^{2}$ where nuclear effect mis-modelling is likely the fit moves the majority of these ratio bins close to unity. 
There are a large number of correlations to consider for this headline fit configuration II and so only a few examples will be shown here. However, further fit parameter contours will be presented in the subsequent discussion of results from fits that were run with slight changes to the headline configuration II. Figures 103 and 104 show the two-dimensional fit contours between the non-flux factor fit parameters (again using the approximation of equation 82). The correlations between the four non-flux factor fit parameters are quite similar to those shown for headline fit I. The main difference is that the muon energy scale, hadronic energy offset and inelastic background scale systematic parameters are not as (anti-)correlated with $M_{A}^{Q E L}$ due to the extra constraints provided by the inclusion of the $E_{\mathrm{v}}^{Q E L}$ distribution. These three systematic parameters are, as before, uncorrelated with each other.
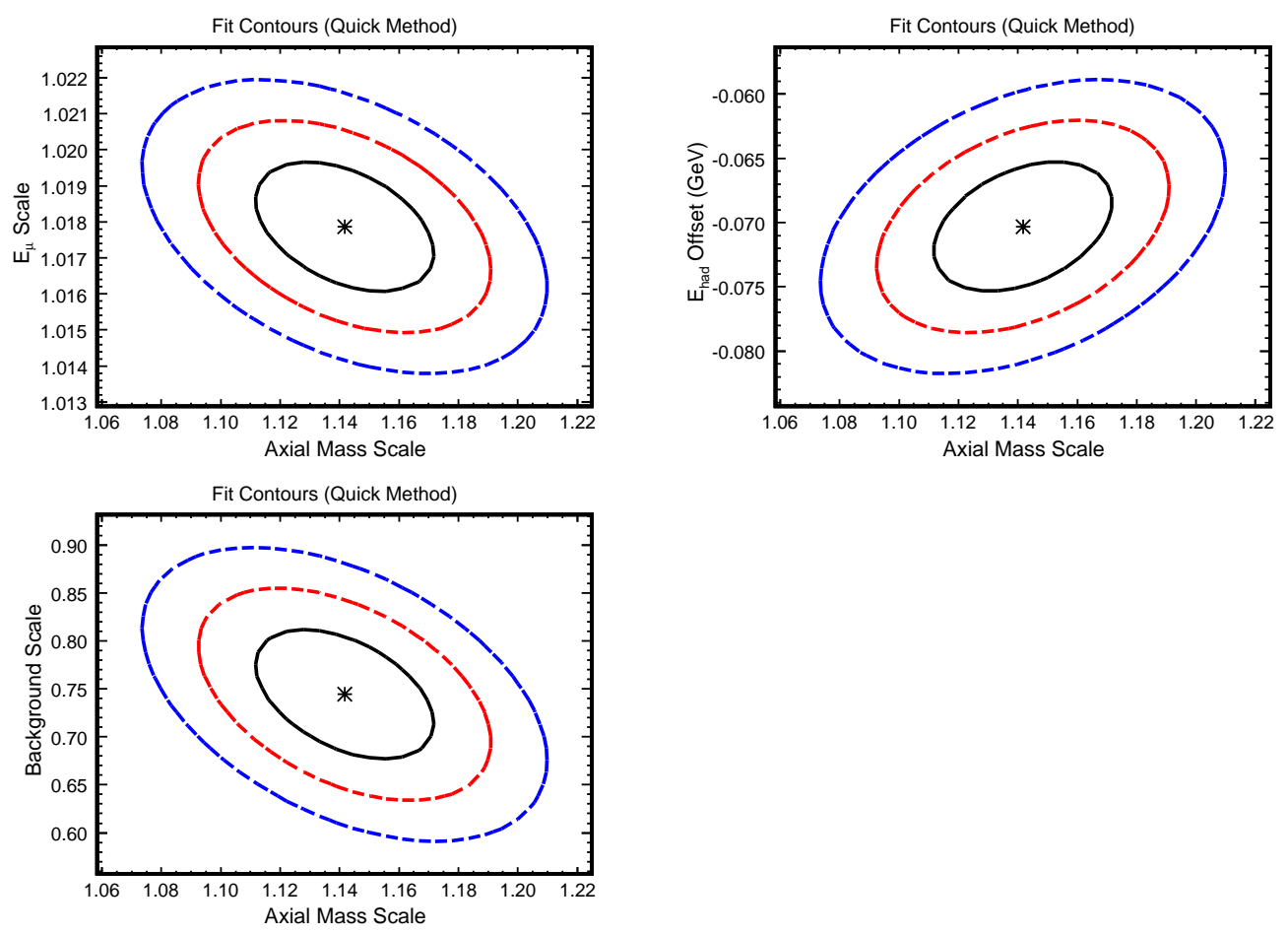

Figure 103: Two-dimensional fit contours for the $M_{A}^{Q E L}$-scale versus each of the three non-flux factor systematic parameters. These contours are made using an elliptical approximation and also use the parameter correlations reported by HESSE which should not be considered accurate to more than $\sim 10$ $20 \%$. The black point shows the best fit values and the black, red and blue ellipses show the $1 \sigma, 2 \sigma$ and $3 \sigma$ contours respectively. 

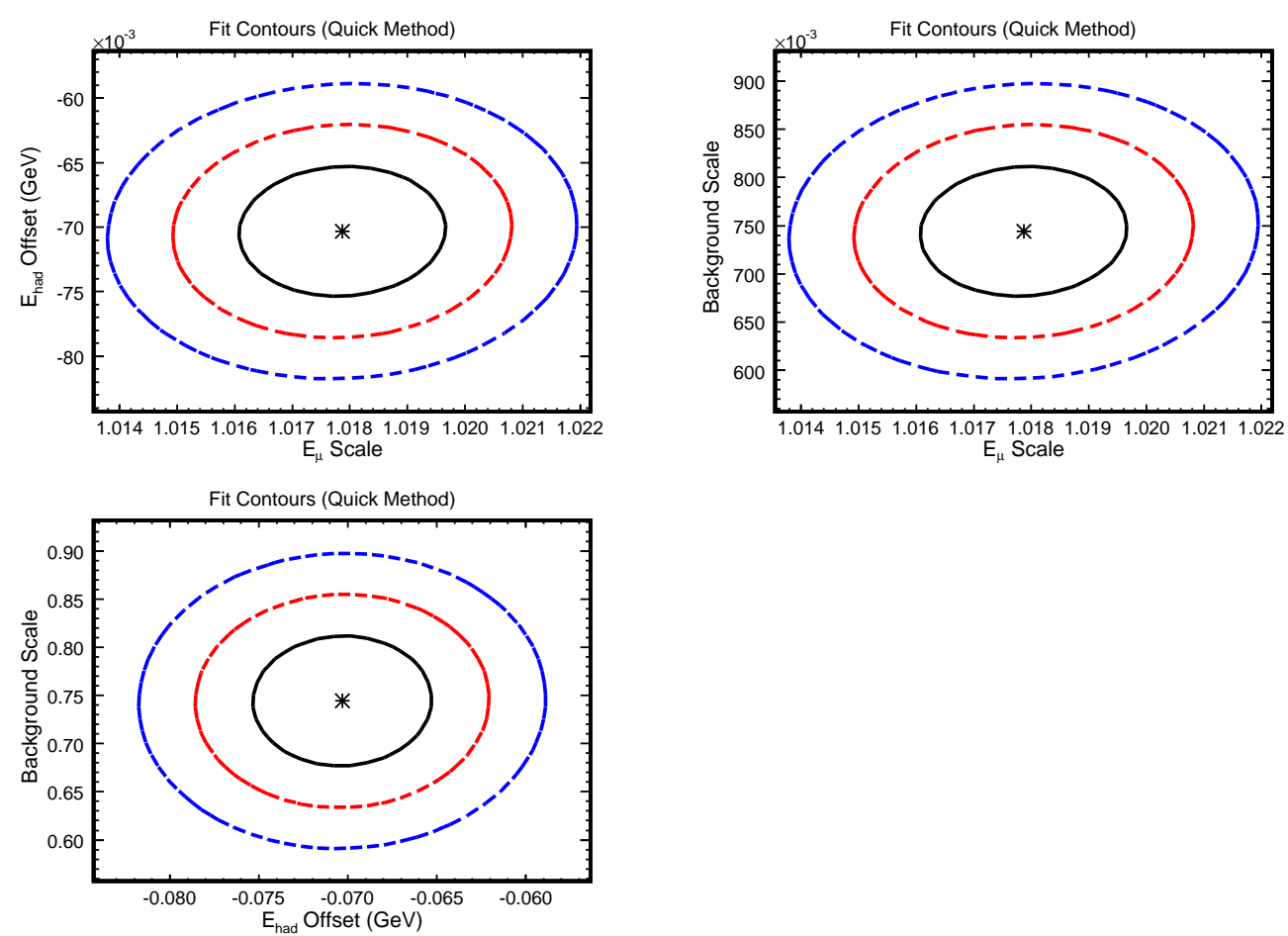

Figure 104: Two-dimensional fit contours for permutations of the three non-flux factor systematic parameters.

A number of fits were also run with small modifications to data fit configuration II in order to check for consistency between the results; the data was fitted with the application of the beam and hadron production tuning weights to the MC and different hadronic energy cuts were used to define the QEL-like sample. In these fits the MINOS routine was not run for any of the fit parameters and so only the symmetric HESSE parameter errors are quoted. Table 18 shows the results of headline fit II to the data with and without the beam and hadron production tuning weights applied to the MC.

Table 18 shows that the results of the fit change quite dramatically when the MC tuning weights are applied. The MC tuning only has a significant effect in the high energy tail of the neutrino energy spectrum where it scales up the MC to better match the data and it can be seen that the fit with MC tuning applied performs as would be expected; the initial $\chi^{2}$ between the data and nominal $\mathrm{MC}$ is lower (better agreement in the tail) and after the fit the higher energy flux factor (from $6 \rightarrow 10 \mathrm{GeV}$ ) is much closer to unity. However, beyond these expected effects it 
can be seen that many of the best fit parameter values are inconsistent between the two fits, most notably $M_{A}^{Q E L}$ and the hadronic energy offset.

Table 19 shows the results from two versions of headline fit II to the data but with the QEL-like sample hadronic energy cut changed to $200 \mathrm{MeV}$ and $300 \mathrm{MeV}$. The results of these two fits are more compatible with the headline fit II results than those from the fit that used the beam and hadron production weights but there are still disagreements between the various fitted parameter values that are larger than the quoted errors.

There are some patterns that emerge when one considers the various results from the permutations of headline configuration II. Firstly, the fitted muon energy scale parameter is consistently $\sim 2 \%$ higher than in the nominal $\mathrm{MC}$ and this is attributed to the extra constraint imposed by the position of the neutrino energy spectrum peak (for these QEL-like events the reconstructed muon energy constitutes the vast majority of the reconstructed neutrino energy). Secondly, whilst the flux factors individually change by large amounts between the fits they do move together. This is perhaps not surprising given that the flux factors were artificially constrained to move together in the construction of the flux factor $\chi^{2}$ penalty term which used correlation coefficients between adjacent flux factors of 0.5 .

It appears that the crudeness of the flux factors is the main problem for these shape and normalisation fits in that they lead to MINUIT focussing more on the neutrino energy spectrum and less on the shape of the $Q_{Q E L}^{2}$ distribution where the main $M_{A}^{Q E L}$ information is to be found. It has been seen that the largest portion of the fit $\chi^{2}$ comes from the differences in the data and MC neutrino energy distribution bin contents and that deviations in adjacent flux factors are responsible for these differences. It is also true that small changes to the flux factors will induce large changes in the $\chi^{2}$ whilst the effect of changes to $M_{A}^{Q E L}$ will be far less pronounced. As such MINUIT is likely to work hard using the flux factors to fit the data neutrino energy spectrum, in particular the peak flux factors where the majority of the events are located, whilst the other fit parameters can be used to 'fine-tune' the minimum. Another associated problem is that with such a large $\chi^{2}$ the systematic parameter penalty term becomes much less important; large changes to the systematic parameters should penalise the statistic heavily but in this fit 


\begin{tabular}{|c||c|c||c|c|}
\hline \multicolumn{1}{|c||}{$\begin{array}{c}\text { Fit } \\
\text { Configuration }\end{array}$} & \multicolumn{2}{c||}{ II } & $\begin{array}{c}\text { II but with beam and hadron } \\
\text { weights applied to MC }\end{array}$ \\
\cline { 2 - 5 } & $\begin{array}{c}\text { Best Fit } \\
\text { Value }\end{array}$ & $\begin{array}{c}\text { HESSE } \\
\text { Error }\end{array}$ & $\begin{array}{c}\text { Best Fit } \\
\text { Value }\end{array}$ & $\begin{array}{c}\text { HES SE } \\
\text { Error }\end{array}$ \\
\hline \hline$M_{A}^{Q E L}(\mathrm{GeV})$ & 1.13026 & 0.01964 & 1.26526 & 0.02434 \\
\hline$E_{\mu}$-Scale & 1.01787 & 0.00119 & 1.01796 & 0.00132 \\
\hline$E_{\text {had }}$-Offset $(\mathrm{GeV})$ & -0.070314 & 0.003327 & -0.016892 & 0.010305 \\
\hline Background Scale & 0.74427 & 0.04457 & 0.70402 & 0.04644 \\
\hline $0-2 \mathrm{GeV}$ Flux Scale & 1.20484 & 0.02931 & 1.29007 & 0.03290 \\
\hline 2-3 GeV Flux Scale & 1.31674 & 0.02262 & 1.37560 & 0.02549 \\
\hline 3-4 GeV Flux Scale & 1.01731 & 0.01444 & 1.06653 & 0.01617 \\
\hline $4-6 \mathrm{GeV}$ Flux Scale & 0.97623 & 0.01585 & 0.96148 & 0.01627 \\
\hline $6-10 \mathrm{GeV}$ Flux Scale & 1.35798 & 0.03124 & 1.05899 & 0.02466 \\
\hline \hline \multicolumn{2}{|c||}{$\chi_{\text {Nom }}^{2}$} & 20227.6 & \multicolumn{3}{c|}{15406.6} \\
\hline$\chi_{B F}^{2}$ & 244.748 & \multicolumn{3}{c|}{212.978} \\
\hline
\end{tabular}

Table 18: Results for data fit configuration II with and without the beam and hadron production tuning weights applied to the MC.

\begin{tabular}{|c|c|c|c|c|}
\hline \multirow[t]{2}{*}{$\begin{array}{c}\text { Fit } \\
\text { Configuration }\end{array}$} & \multicolumn{2}{|c|}{$\begin{array}{l}\text { II but with hadronic } \\
\text { energy cut at } 200 \mathrm{MeV}\end{array}$} & \multicolumn{2}{|c|}{$\begin{array}{l}\text { II but with hadronic } \\
\text { energy cut at } 300 \mathrm{MeV}\end{array}$} \\
\hline & $\begin{array}{l}\text { Best Fit } \\
\text { Value }\end{array}$ & $\begin{array}{l}\text { HESSE } \\
\text { Error }\end{array}$ & $\begin{array}{l}\text { Best Fit } \\
\text { Value }\end{array}$ & $\begin{array}{l}\text { HESSE } \\
\text { Error }\end{array}$ \\
\hline$\overline{M_{A}^{Q E L}(\mathrm{GeV})}$ & 1.07011 & 0.01168 & 1.16359 & 0.01703 \\
\hline$E_{\mu}$-Scale & 1.01828 & 0.00154 & 1.01773 & 0.00101 \\
\hline$E_{\text {had }}$-Offset $(\mathrm{GeV})$ & -0.117808 & 0.003637 & -0.045699 & 0.002964 \\
\hline Background Scale & 0.75590 & 0.04122 & 0.73210 & 0.04594 \\
\hline 0-2 GeV Flux Scale & 1.12290 & 0.02861 & 1.27327 & 0.03162 \\
\hline 2-3 GeV Flux Scale & 1.23874 & 0.02253 & 1.38142 & 0.02420 \\
\hline 3-4 GeV Flux Scale & 0.97000 & 0.01404 & 1.05287 & 0.01534 \\
\hline 4-6 GeV Flux Scale & 0.93606 & 0.01541 & 1.01324 & 0.01678 \\
\hline 6-10 GeV Flux Scale & 1.28169 & 0.03017 & 1.42498 & 0.03294 \\
\hline$\overline{\chi_{N o m}^{2}}$ & \multicolumn{2}{|c|}{19314.6} & \multicolumn{2}{|c|}{21321.5} \\
\hline$\chi_{B F}^{2}$ & \multicolumn{2}{|c|}{244.169} & \multicolumn{2}{|c|}{270.391} \\
\hline
\end{tabular}

Table 19: Results for data fit configuration II with the QEL-like sample hadronic energy cut at $200 \mathrm{MeV}$ and $300 \mathrm{MeV}$. 
can be easily compensated by very small changes in the flux factors.

Under the hypothesis that the fit primarily focusses on the neutrino energy spectrum, in particular in the peak, it is now interesting to consider the correlations of the flux factors with the other fit parameters to see if the observed fitted parameter value deviations (as evidenced in tables 17-19) should be expected. Figure 105 shows two-dimensional contours between a selection of flux factors using the elliptical approximation and the results from headline fit II. It shows that the flux factors are positively correlated with each other, as should be expected given that the correlation matrix was put in by hand to the flux factor penalty term part of the fit $\chi^{2}$. As such the subsequent figure 106 only shows the fit contours between a peak flux factor (from $2 \rightarrow 3 \mathrm{GeV}$ ) and the non-flux factor fit parameters but does illustrate the general forms of the correlations between all of the flux factors and the remaining fit parameters.

Figure 106 shows that the flux factors are only strongly correlated, in this case anti-correlated, with the background scale parameter and figures 103 and 104 showed that in turn the background scale is only strongly correlated with $M_{A}^{Q E L}$. Then, assuming that the muon energy scale parameter is fairly well constrained, changes in $M_{A}^{Q E L}$ can be accomodated by changes to the hadronic energy offset, which itself is not tightly correlated with any of the other fit parameters. Considering these correlations it is plausible that there is a lot of play between the flux in the energy spectrum peak, the background scale, the value of $M_{A}^{Q E L}$ and the hadronic energy offset and that the large fit $\chi^{2}$, coming from the flux factors and the neutrino energy spectrum, allows for movements in all of these parameters without appreciable penalty.

The above discussion shows that the shape and normalisation fit to both the $Q_{Q E L}^{2}$ and $E_{\mathrm{v}}^{Q E L}$ distributions is sensitive to the systematic parameters and suffers from the crude nature of the flux factors themselves with the fit results changing beyond what may be expected, given the returned parameter errors, when slightly different configurations are used. For a consistent $M_{A}^{Q E L}$ to be reported by the above permutations of headline fit II the systematic parameters would need to be better understood (and hence more strongly constrained in the $\chi^{2}$ penalty term) and in particular the flux factors would need to be replaced with a more sophisticated treatment of the uncertainties in the incident neutrino flux. However, all of the fits do agree 

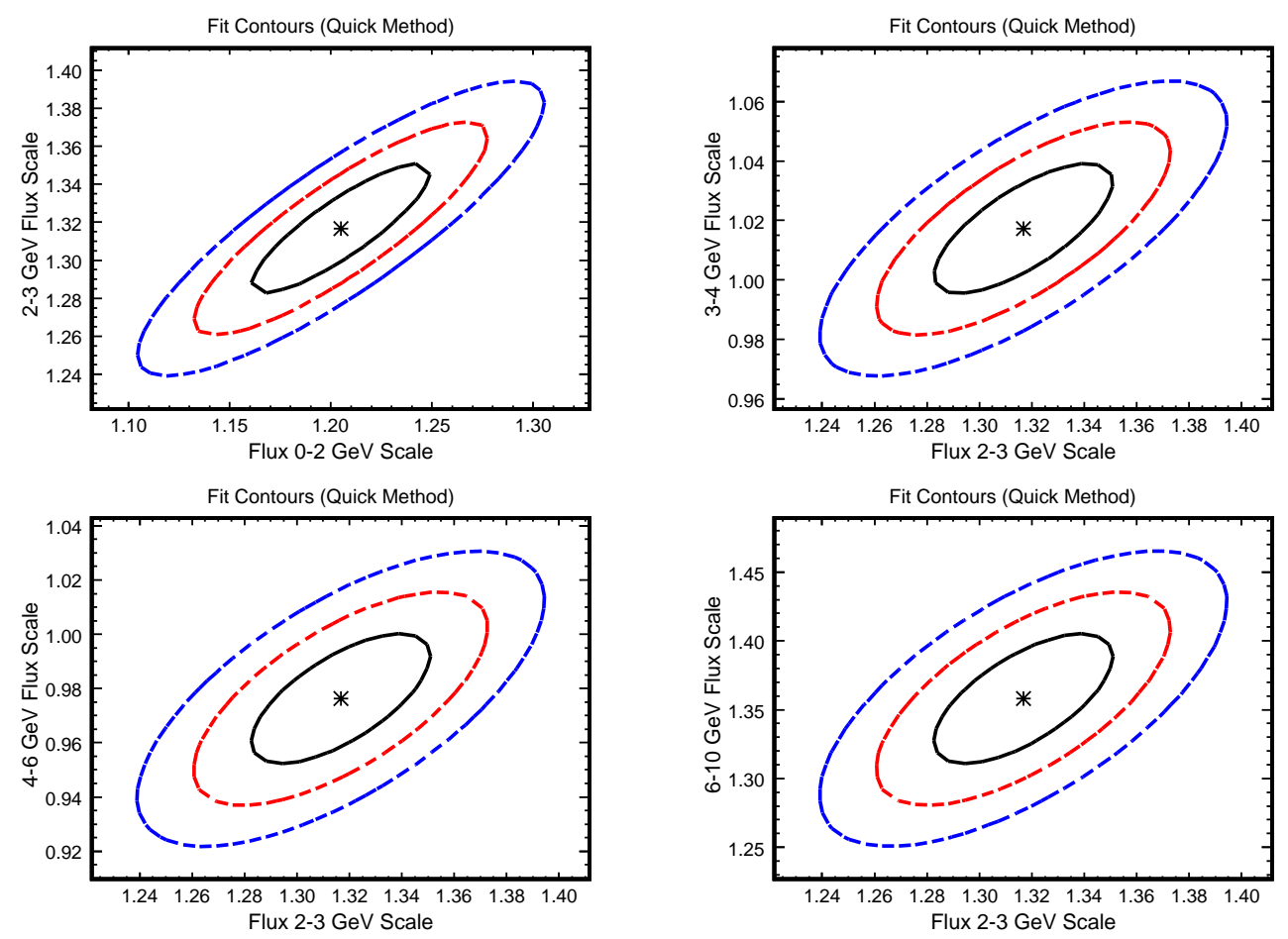

Figure 105: Two-dimensional fit contours for a selection of flux factors. These contours are made using an elliptical approximation and also use the parameter correlations reported by HESSE for the results from headline fit II (which should not be considered accurate to more than 10-20\%). The black point shows the best fit values and the black, red and blue ellipses show the $1 \sigma, 2 \sigma$ and $3 \sigma$ contours respectively. It should be noted that the a priori correlation coefficients between the flux factors were put into the fit $\chi^{2}$ by hand.

that the value of $M_{A}^{Q E L}$ should be increased in the MC from the default value of $0.99 \mathrm{GeV}$ and suggest that this increase should be between $\sim 10 \%$ to $\sim 30 \%$.

As with the result from the configuration I fits, equation 93 notes statistical errors which are given by the MINOS errors from headline fit II and systematic errors that are given by the largest deviations in the measured $M_{A}^{Q E L}$ value using the fits with different permutations of the fit configuration. In this case the largest upward shift comes from the fit where the beam and hadron production weights were applied to the MC and the largest downward shift from the fit where the QEL-like sample was defined by a cut on the hadronic energy at $200 \mathrm{MeV}$. It should again be noted that this statistical error contains a non-statistical component that is due to the inclusion of the systematic parameters used in the fits (particularly the muon energy scale) 

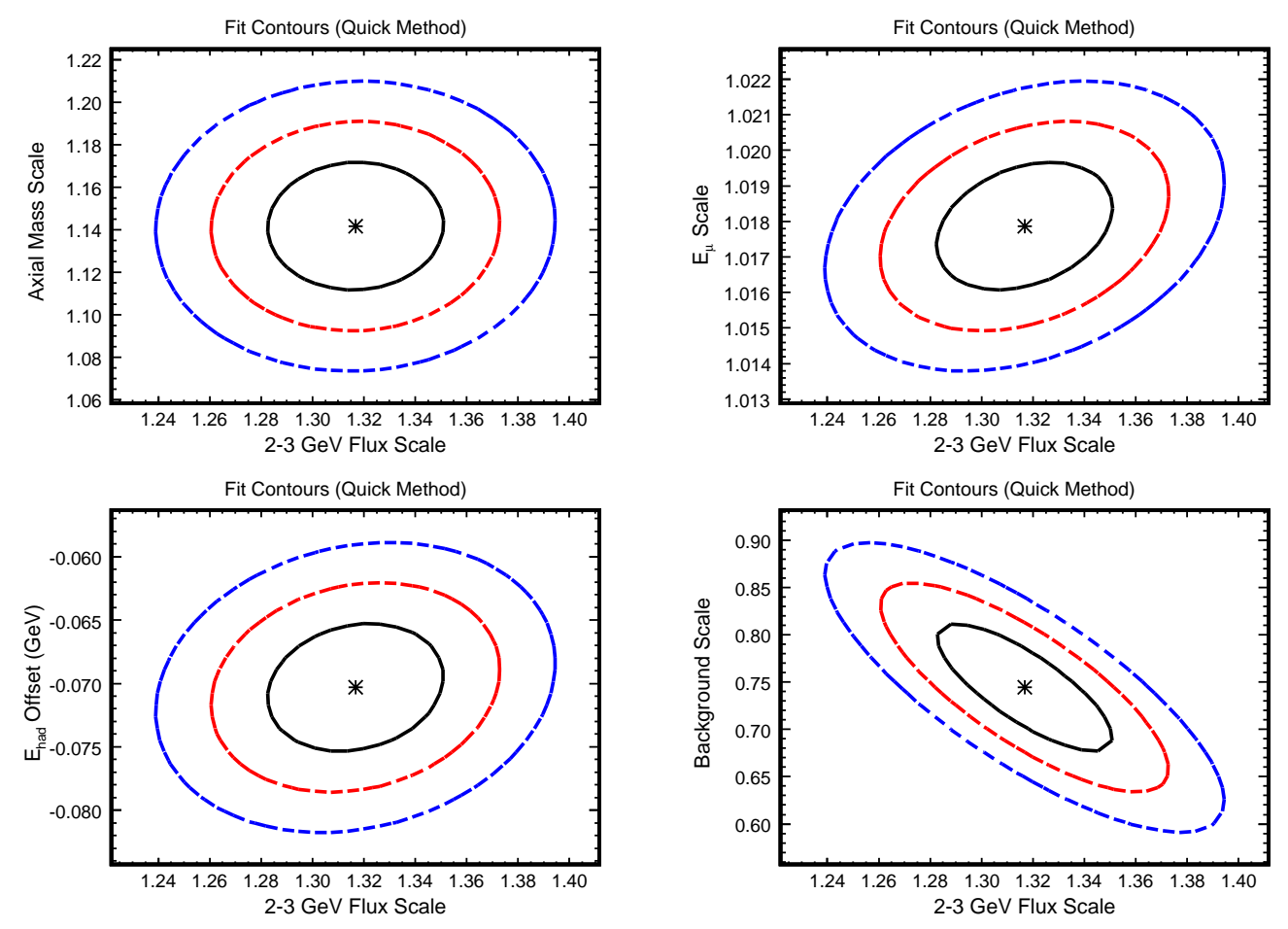

Figure 106: Two-dimensional fit contours as a function of the $2 \rightarrow 3 \mathrm{GeV}$ flux factor and each of the non-flux factor systematic parameters. These contours are made using an elliptical approximation and also use the parameter correlations reported by HESSE for the results from headline fit II (which should not be considered accurate to more than $\sim 10-20 \%)$. The black point shows the best fit values and the black, red and blue ellipses show the $1 \sigma, 2 \sigma$ and $3 \sigma$ contours respectively.

and likewise that this systematic error contains a statistical component due to the fact that it is calculated using alternate fits to the data.

$$
\left.\left.M_{A}^{Q E L}=1.130+0.021 \text { (stat. }\right)_{-0.060}^{+0.135} \text { (syst. }\right) \mathrm{GeV}
$$

\subsection{Reconciling the Fit Results}

The previous section presented detailed comments on each of the headline fits individually whereas this section will try to reconcile the differences between the results obtained using the two headline fit configurations. The various fits to the data, in particular the results quoted in equations 92 and 93, show that in general the shape-only fits to the $Q_{Q E L}^{2}$ distribution result in a higher best fit $M_{A}^{Q E L}$ than for the results of the shape and normalisation fits to both the $Q_{Q E L}^{2}$ 
and $E_{\mathrm{v}}^{Q E L}$ distributions.

In both fit types there is a large anti-correlation between $M_{A}^{Q E L}$ and the muon energy scale and the results of headline fit II show a much lower $M_{A}^{Q E L}$ value and a much higher muon energy scale value (than the results of headline fit I) in occordance with this correlation. Furthermore, it can be seen that the anomalous local minimum results from the shape-only fit performed in the neutrino energy slice from $2.0 \rightarrow 3.0 \mathrm{GeV}$ are in much better agreement with those returned by the type II fits and that with this set of best fit parameter values there is a flattening of the data over MC ratio as a function of $Q_{Q E L}^{2}$ (see figure 97). However, it can also be seen from figure 97 that when absolute normalisation is applied then this set of parameter values are not capable of filling-in the MC deficit. In the type II fits, that also consider both the $E_{\mathrm{v}}^{Q E L}$ distribution and normalisation information, the lower $M_{A}^{Q E L}$ values and higher muon energy scales produce just such a flattening but now the additional fit parameters (the hadronic energy offset, background scale and flux factors), which have little effect on the shape of the $Q_{Q E L}^{2}$ distribution, are used to correct the remaining difference in normalisation. Finally the large $\chi^{2}$ values seen in the type II fits, stemming from the underlying discrete nature of the flux factors, mean that these additional 'normalisation fixing' parameters are all but free to move to whatever values are necessary.

The above interpretation explains the difference between the results from fits of type I and II in terms of the different parameter sets used in the fit, the additional normalisation and neutrino energy distribution information used in the type II fits and the different parameter correlations and constraints. In particular, it seems that if one could more tightly constrain (or even fix) the muon energy scale systematic parameter then both fit types could arrive at a consistent $M_{A}^{Q E L}$ value in order to appropriately adjust the $\mathrm{MC} Q_{Q E L}^{2}$ distribution. Some additional fits were run in each configuration to test this hypothesis; firstly the muon energy scale assumed $1 \sigma$ error was reduced from $2.0 \%$ to $1.0 \%$ or $0.5 \%$ (which are perhaps not unreasonable values given the recent work looking at low energy muons in CalDet) and secondly fits were run where the muon energy scale was pinned to particular values.

Table 20 shows the results from headline fit I along with fit results from identical type I fits 
but using (a) an assumed $1 \sigma$ error on the muon energy scale of $1.0 \%$, (b) an assumed $1 \sigma$ error on the muon energy scale of $0.5 \%$, (c) a fixed muon energy scale set to the best fit value from headline fit II and (d) a fixed muon energy scale set to a value of unity. Table 21 shows the results from headline fit II along with fit results from identical type II fits but using (a) an assumed $1 \sigma$ error on the muon energy scale of $1.0 \%$, (b) an assumed $1 \sigma$ error on the muon energy scale of $0.5 \%$, (c) a fixed muon energy scale set to the best fit value from headline fit I and (d) a fixed muon energy scale set to a value of unity. Neither HESSE nor MINOS errors are quoted in these tables although it is the central values for each of the fit parameters that are of interest here.

\begin{tabular}{|c|c|c|c|c|c|}
\hline $\begin{array}{c}\text { Fit } \\
\text { Configuration }\end{array}$ & $\begin{array}{c}M_{A}^{Q E L} \\
(\mathrm{GeV})\end{array}$ & $E_{\mu}$-Scale & $\begin{array}{c}E_{\text {had }} \text {-Offset } \\
(\mathrm{MeV})\end{array}$ & Bg-Scale & $\chi_{B F}^{2}$ \\
\hline \hline I & 1.3862 & 0.9789 & -32.304 & 0.9794 & 8.425 \\
\hline I (a) & 1.3992 & 0.9804 & -21.798 & 0.9591 & 11.869 \\
\hline I (b) & 1.2721 & 0.9981 & -13.473 & 0.9026 & 17.518 \\
\hline I (c) & 1.1161 & 1.0179 & -15.131 & 0.8123 & 15.728 \\
\hline I (d) & 1.2574 & 1.0000 & -13.286 & 0.8958 & 17.591 \\
\hline
\end{tabular}

Table 20: Results for data fit configuration I with a series of tigher constraints upon the muon energy scale. The labels $(a)-(d)$ are defined in the above text.

Table 21 shows that reducing the size of the assumed $1 \sigma$ error on the muon energy scale has little effect on the type II fitted $M_{A}^{Q E L}$ value. This is because the fit $\chi^{2}$ is dominated by the flux factor-induced discontinuities in the bin contents of the neutrino energy distribution and so the extra penalty when the muon energy scale error is reduced is negligible. Table 20 shows that reducing the assumed error on the muon energy scale to $1 \%$ also does not have a large effect on the type I fitted $M_{A}^{Q E L}$ value. However, when the assumed error is reduced to $0.5 \%$ there is a noticable effect on the fit I results with the best fit muon energy scale closer to unity and the best fit $M_{A}^{Q E L}$ becoming lower in accordance with the anti-correlation between these parameters.

Encouragingly the tables do show that the two types of fit agree upon the value of $M_{A}^{Q E L}$ when the muon energy scale is fixed. In particular, when the muon energy scale is fixed to unity both 


\begin{tabular}{|c|c|c|c|c|c|}
\hline $\begin{array}{c}\text { Fit } \\
\text { Configuration }\end{array}$ & II & II (a) & II (b) & II (c) & II (d) \\
\hline \hline$M_{A}^{Q E L}(\mathrm{GeV})$ & 1.1303 & 1.0852 & 1.0628 & 1.3755 & 1.2622 \\
\hline$E_{\mu}$-Scale & 1.0179 & 1.0173 & 1.0099 & 0.9789 & 1.0000 \\
\hline$E_{\text {had }}$-Offset $(\mathrm{MeV})$ & -70.314 & -90.812 & -123.685 & -89.436 & -70.927 \\
\hline Background Scale & 0.7443 & 0.7385 & 0.6824 & 0.5987 & 0.6832 \\
\hline 0-2 GeV Flux Scale & 1.2048 & 1.1748 & 1.1211 & 1.0042 & 1.1142 \\
\hline 2-3 GeV Flux Scale & 1.3167 & 1.2959 & 1.2471 & 1.1403 & 1.2365 \\
\hline 3-4 GeV Flux Scale & 1.0173 & 1.0076 & 0.9970 & 0.9837 & 1.0031 \\
\hline 4-6 GeV Flux Scale & 0.9762 & 0.9708 & 0.9869 & 1.0447 & 1.0087 \\
\hline 6-10 GeV Flux Scale & 1.3580 & 1.3453 & 1.3476 & 1.4028 & 1.3784 \\
\hline \hline$\chi_{B F}^{2}$ & 244.748 & 243.984 & 255.186 & 395.963 & 275.734 \\
\hline
\end{tabular}

Table 21: Results for data fit configuration II with a series of tigher constraints upon the muon energy scale. The labels $(a)-(d)$ are defined in the above text.

fit types return an $M_{A}^{Q E L}$ value of $\sim 1.25 \mathrm{GeV}$ and when the muon energy scale is fixed to the best fit value from headline fit I the type II fit returns a consistent best fit $M_{A}^{Q E L}$ (and vice versa).

As a final check a type II (shape and rate) fit was performed where the flux factors were all kept constant and in this case the best fit muon energy scale was 0.982 and the best fit $M_{A}^{Q E L}$ was $1.285 \mathrm{GeV}$. The results of this final type II fit show that when the fit cannot use the flux factors to force unphysical warpings of the neutrino energy distribution the prefered parameter values are in much better agreement with those returned by headline fit I.

\subsection{Summary and Final Result}

Fits for the quasi-elastic axial-vector mass were performed in two possible configurations; to the shape of the $Q_{Q E L}^{2}$ distribution (type I) and to both the shape and rate of the $Q_{Q E L}^{2}$ and $E_{\mathrm{v}}^{Q E L}$ distributions (type II). The results of the type II fits have been discredited due to the crude nature of the flux factor parameters and furthermore it has been seen that when either the flux factors are fixed or the muon energy scale parameter is fixed the best fit axial-mass values from the type II fits are brought into agreement with those returned by the type I fit. 
As such, the result of the headline type I fit is presented as the final result of the work described in this thesis. However, due to the large anti-correlation between $M_{A}^{Q E L}$ and the muon energy scale that has been seen to influence the results of all the fits presented in this chapter, this final result is not presented as a single number for $M_{A}^{Q E L}$ but rather as a set of confidence interval contours in the two-dimensional space of $\left(M_{A}^{Q E L}\right.$-scale, $E_{\mu}$-scale). Figure 107 shows the $1 \sigma, 2 \sigma$ and $3 \sigma$ confidence interval contours (calculated by looking for changes in the fit $\chi^{2}$ of 2.30, 6.18 and 11.83 respectively) corresponding to headline fit I where at each point on each contour the $\chi^{2}$ has been minimised with respect to the other two fit parameters; the hadronic energy offset and the background scale.

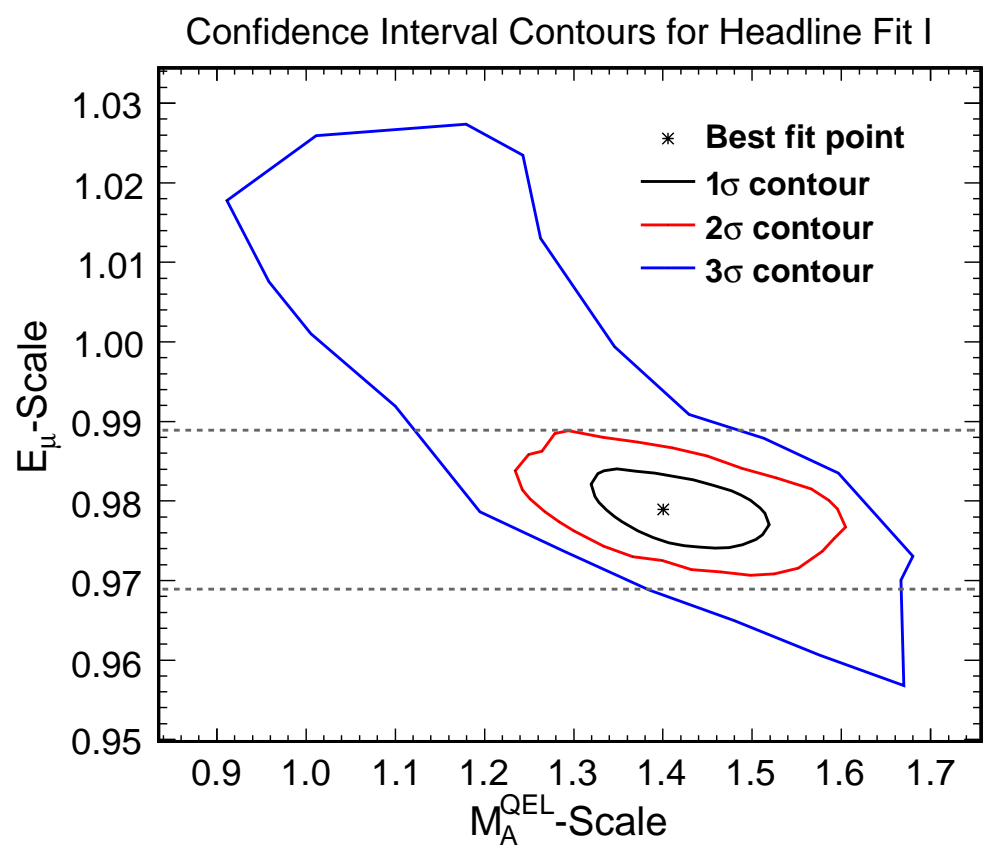

Figure 107: $1 \sigma, 2 \sigma$ and $3 \sigma$ confidence interval contours for headline fit I. At each point on each contour the fit $\chi^{2}$ has been minimised with respect to the other two fit parameters; the hadronic energy offset and the background scale. The x-axis shows the $M_{A}^{Q E L}$-scale, as used directly in the fits, and can be converted to an $M_{A}^{Q E L}$ value in $\mathrm{GeV}$ by multiplying by the default MC value of $0.99 \mathrm{GeV}$. The dashed lines show $\pm 1 \%$ changes to the muon ennergy scale about the best fit point.

It can be seen from figure 107 that both the $1 \sigma$ and $2 \sigma$ contours can be closed into an elliptical shape but that this is not the case for the $3 \sigma$ contour. In the case of the $3 \sigma$ contour the unusual shape is very similar to that shown in figure 99 and there is not a sufficient change in $\chi^{2}$ 
for this contour to be closed into an elliptical shape. Instead, this contour ranges-out in a direction consistent with the anti-correlation between these fit parameters and around the region of parameter space suggested by the best fit results from the type II fits.

As such the type II results are not excluded at $3 \sigma$ although the nominal MC values, which in the parameter space of figure 107 occur at $(1.0,1.0)$, are excluded at the $3 \sigma$ confidence level. The best fit axial mass value is $1.386 \mathrm{GeV}$ corresponding to a muon energy scale of 0.979 and it can be seen from the $\pm 1 \%$ changes to the muon energy scale lines from figure 107 that the muon energy scale would have to be understood to a much higher level of accuracy for the value of $M_{A}^{Q E L}$ to be tightly constrained.

\subsection{Improvements for the Future}

The potential improvements for this measurement come in three main forms; improvements to the fitting method, increased understanding of the systematic parameters and the use of additional sources of information.

A very flexible and robust fit has been developed and the major advance that could now aid the results is a better treatment of the uncertainty in the incident neutrino flux. It has been demonstrated that the main problem with the shape-only fits to the $Q_{Q E L}^{2}$ distribution is the anticorrelation between $M_{A}^{Q E L}$ and the muon energy scale. It was hoped that by additionally fitting the $E_{\mathrm{v}}^{Q E L}$ distribution this correlation could be reduced however any fit to the neutrino energy spectrum must be able to account for the uncertainties in the neutrino flux incident on the ND. There are two main possibilities for this improvement; a more sophisticated treatment of the existing flux factors or replacing them with the parameters used in the MC tuning procedure mentioned in chapter 6 :

- Interpolated flux factors: the main problem with the existing flux factor parameters is their discrete nature; moving adjacent pieces of the true neutrino energy spectrum creates a discontinuity and when considering the reconstructed neutrino energy spectrum such a discontinuity is not totally smeared out but leaves a residual sinusoidal warping. One relatively simple extension would be to interpolate between the scale factors at the 
centres of adjacent flux factors and then apply a weight to an event based on the value of this interpolated scale at the value of true neutrino energy taken by the event. Such a procedure might smooth the effect on the reconstructed neutrino energy distribution of deviations between adjacent flux factors.

- MC tuning parameters: a second and more sophisticated option would be to include the beam and hadron production weighting parameters, as described in chapter 6 , directly into the fit for $M_{A}^{Q E L}$. This option would make the fit more complicated but has the advantage that the a priori errors on the beam optics parameters (although not the hadron production parameters) are known and that all of the parameters have a more physically meaningful interpretation than scaling pieces of the true neutrino energy distribution.

In general an increased understanding of all of the systematic parameters would allow for tighter assumed $1 \sigma$ errors to be used in the construction of the penalty term to the fit $\chi^{2}$ which may help to remove some of the degeneracy between the various sets of fit parameter results. The following presents some possible avenues for further work with the systematic parameters:

- Muon energy scale: the assumed $1 \sigma$ error, used in the penalty term for the fit $\chi^{2}$, for this parameter was calculated using the full CC-like event sample. It is likely that the uncertainty in the muon energy is smaller for the QEL-like event sample where there are no large hadronic systems to confuse the muon tracking and vertex finding. This effect was demonstrated in chapter 6 when the ND kinematic resolutions were discussed and some further analysis could allow for a smaller assumed $1 \sigma$ error to be evaluated.

The quoted uncertainty of $2 \%$ was calculated using events with both stopping and exiting muons together and it could be that the negative effect of a loss in sample statistics, when only events with stopping muons are used in the analysis, could be offset by a reduced uncertainty in the muon energy scale for muons whose momentum is measured by range in the detector. The uncertainty on the range-based measurement could be reduced by further analysis of CalDet data, such as mentioned in chapter 7, or by a more accurate accounting for the mass of the detector.

- Hadronic energy offset: MINOS has recently been updating it's modelling of intranuclear rescattering and in particular a reweighting procedure has been developed to 
facilitate detailed studies of the effects of model (and model parameter value) changes. This reweighting opens the possibility of detailed studies of the effect of FSIs on the QEL-like event sample and some of the more important parameters, for the $M_{A}^{Q E L}$ measurement, could be included directly as fit parameters to provide a more physically accurate way of incorporating the uncertainty in the energy of the hadronic system.

- Inelastic background scale: it was mentioned previously that the background scale was a crude way to consider the uncertainties in the level of the RES and DIS cross sections and could not address uncertainties in the cross section shapes as a function of $Q^{2}$ and $E_{\mathrm{v}}$. The fit could be adapted to use more physically meaningful parameters such as the resonant event axial-vector mass, $M_{A}^{1 \pi}$, and changes to the parameters describing the modified KNO scaling used in the modelling of DIS events.

The final area in which the $M_{\tilde{A}}^{Q E L}$ analysis could be improved is in the utilisation of additional sources of information. The analysis has not yet considered exiting muons, events from alternate beam configurations or anti-neutrino interactions. The following list discusses the possible implications of using such information:

- Exiting muons: uncertainties in the calibration of the magnetic field meant that interactions where the produced muon exited the detector (and where the momentum was measured by curvature) were not considered for this analysis. MINOS is currently evaluating a new calibration of the field and, when it is sufficiently understood, in the future the exiting muon events could also be used in the $M_{A}^{Q E L}$ extraction. This will benefit the analysis both by increasing the sample statistics and by adding events with higher reconstructed neutrino energies (there are virtually no stopping muon events with $E_{\mathrm{v}}^{\text {reco }}>10$ $\mathrm{GeV}$ ) and higher reconstructed squared four-momentum transfers.

- Alternate beam configurations: MINOS has taken data in a number of different beam configurations and in particular there are substantial datasets that have been collected using the higher energy L100z200i and L250z200i configurations. Firstly it would be interesting to repeat the $M_{A}^{Q E L}$ extraction for each of these independant datasets although the statistical error on the measurement would be larger due to the reduced exposures (compared to the L010z185 configuration) as well as the reduced fraction of true QEL 
events (the DIS cross section dominates as the neutrino energy increases). A second use for this data is to provide further constraints on the flux in the fits; the different beam configurations produce neutrinos whose parent hadrons come from different regions of hadron $\left(p_{z}, p_{T}\right)$-space. Data from all the beam configurations could be fitted for a single $M_{A}^{Q E L}$ (and single systematic parameters) but with the fitted flux parameters constrained by the information from the different beam configurations. The current flux factors would probably not work in this case but a more sophisticated treatement, such as via the beam optics and hadron production parameters, could be appropriate for such a fit.

- Anti-neutrino interactions: in the current analysis anti-neutrino interactions are removed using a cut on the muon charge-sign. It would be interesting to repeat the $M_{A}^{Q E L}$ extraction for the anti-neutrino sample to check for consistenty and it could also be advantageous to include these events in a combined analysis for extra statistical power. However, both the inclusive and exclusive CC cross sections for anti-neutrino interactions are not well constrained by experimental data and the large uncertainties could result in large errors on the fit results. 


\section{Conclusion}

The field of neutrino physics is a fascinating and incredibly active area of particle physics research which offers some unique insights into the way our universe works. In the last decade the discovery of neutrino oscillations confirmed that neutrinos must possess mass and high precision measurements of some of the parameters governing these oscillations have been made. The MINOS experiment continues to reduce the uncertainty on the parameters governing atmospheric neutrino oscillations and is in a good position to set a competitive limit on, or even discover, the sub-dominant oscillation of muon neutrinos to electron neutrinos.

All measurements of neutrino oscillations are underpinned by our knowledge of the way neutrinos interact with matter and as the next generation of neutrino experiments is born the uncertainties on the cross sections for neutrino scattering will need to be significantly reduced. The MINOS Near Detector records a huge rate of neutrino interactions and has collected well over three million events since the start of NuMI beam operation in 2005. This large data sample provides MINOS with an opportunity to make some of the most precise measurements of neutrino interaction cross sections, in the few-GeV regime, that have ever been made.

Of particular interest is neutrino quasi-elastic scattering which is not only the most prominent interaction type in the few-GeV regime (where the next generation of neutrino experiments will operate) but also can probe the axial nature of the weak interaction with nucleons and provide a window into the highly complex world of nuclear effects. Furthermore, there is considerable uncertainty in the exclusive cross section for quasi-elastic scattering with a variety of previous measurements disagreeing by up to $30 \%$.

Theoretically quasi-elastic scattering is described using a number of nucleon form factors which describe how much of each possible type of weak current contributes to a scatter as a function of the squared four-momentum transfer between the leptonic and hadronic vertices. Experimental observations tightly constrain all but one of these form factors; the axial-vector form factor. When parameterised using a dipole form, the single unknown parameter in the expression for the axial-vector form factor (and hence in the cross section for quasi-elastic scattering) is the axial-vector mass, $M_{A}^{Q E L}$, and the $Q^{2}$ evolution of which can only be extracted 
using neutrino scattering data.

A method to select a quasi-elastic enriched sample of events from the MINOS Near Detector was presented and a highly versatile and robust fitting procedure was developed to use the $Q_{Q E L}^{2}$ and $E_{\mathrm{v}}^{Q E L}$ distributions of the quasi-elastic enriched event sample to extract $M_{A}^{Q E L}$ from MINOS data. The fit includes a number of the most important systematic uncertainties directly and has been shown to perform well in mock data studies. The fit can be run in two headline configurations and the results from fits to the Near Detector data were presented for each of these configurations.

The fit results obtained using these alternate configurations were shown not to agree and it was demonstrated that the fits to both the $Q_{Q E L}^{2}$ and $E_{\mathrm{v}}^{Q E L}$ distributions suffered from an insufficient treatment of the uncertainties in the neutrino flux incident on the Near Detector. As such the shape of the $Q_{Q E L}^{2}$ distribution was fit to result in an axial-vector mass value of $1.386 \mathrm{GeV}$ with a corresponding muon energy scale value of 0.979 . This best fit result is $40 \%$ above the nominal MINOS Monte Carlo value of $0.99 \mathrm{GeV}$ although in this fit configuration the large anti-correlation between the axial-vector mass and the muon energy scale meant that the result was presented in the form of a set of two-dimensional confidence interval contours which span a fairly large range of $M_{A}^{Q E L}$ values.

A number of potential improvements to the fitting method were proposed and, given that the measurement is not limited by the statistical error, the focus of further work will be to improve the understanding and treatment of the systematic parameters. As the fitting method becomes more sophisticated and robust the huge number of neutrino interactions recorded in the MINOS Near Detector may provide the level of sensitivity required for MINOS to start to address some of the more elusive questions in the modelling of quasi-elastic scattering; is the dipole approximation for the axial-vector form factor correct and can low $Q^{2}$ nuclear effects be understood and accurately modelled?

Low energy neutrino-nucleus scattering is an engrossing and highly rewarding area of research that requires a detailed understanding of phenomenology and input from a great variety of 
particle physics experiments. Neutrino-nucleus interaction cross sections need to be understood at an ever increasing level of accuracy to facilitate the next generation of neutrino oscillation measurements and the author has no doubt that they will continue to confound and intrigue particle physicists for many years to come. 


\section{References}

[1] W. Pauli, Letter reproduced in translation, Physics Today (1978)

[2] F. Reines and C. L. Cowan, Phys. Rev. 92, 830 (1953)

[3] R. Becker-Szendy et al. [IMB Collaboration], Phys. Rev. D 46 (1992) 3720

[4] K. S. Hirata et al. [Kamiokande II Collaboration], Phys. Lett. B 280 (1992) 146

[5] W. W. Allison et al. [Soudan-2 Collaboration], Phys. Lett. B 449 (1999) 137

[6] B. Pontecorvo, Sov. Phys. JETP 26 (1968) 984 [Zh. Eksp. Teor. Fiz 53 (1967) 1717]

[7] Y. Fukuda et al. [Super-Kamiokande Collaboration], Phys. Rev. Lett. 81 (1998) 1562

[8] D. G. Michael et al. [MINOS Collaboration], Phys. Rev. Lett. 97 (2006) 191801

[9] Z. Maki, M. Nakagawa and S. Sakata, Prog. Theor. Phys. 28 (1962) 870

[10] G. Fogli, E. Lisi and G. Scioscia, Phys. Rev. D 52 (1995) 5334

[11] P. Adamson et al. [The MINOS Collaboration], hep-ex/07110769 (2007), Submitted to Phys. Rev. D

[12] L. Wolfenstein, Phys. Rev. D 17 (1978) 2369

[13] S. Mikheyev and A. Smirnov, Sov. J. Nucl. Phys 42 (1986) 913 [Yad. Fiz. 42 (1985) 1441]; Sov. Phys. JETP 64 (1986) 4 [Zh. Eksp. Teor. Fiz 91 (1986) 7]

[14] T. D. Lee and C. N. Yang, Phys. Rev. 104 (1956) 254-258

[15] C. S. Wu et al., Phys. Rev. 105 (1957) 1413

[16] M. Goldhaber et al., Phys. Rev. 109 (1958) 1015

[17] S. L. Glashow, Nucl. Phys. 22579

[18] S. Weinberg, Phys. Rev. Lett. 191264

[19] A. Salam, Elementary Particle Physics ed N. Svartholm (Stockholm: Almqvist and Wiksells) 
[20] F. J. Hasert et al., Phys. Lett. B 46 (1973) 138-140

[21] R. P. Feynman and M. Gell-Mann, Phys. Rev. 109 (1958) 193

[22] M. Gell-Mann and M. Levy, Nuovo Cimento 16 (1960) 705

[23] C. H. Llewellyn Smith, Phys. Rep. 3 (1972) 261

[24] S. M. Bilenky, Introduction to the Physics of Electroweak Interactions (Pergamon Press)

[25] M. N. Rosenbluth, Phys. Rev. 79 (1950) 615-619

[26] R. G. Sachs, Phys. Rev. Lett. 12 (1964) 231

[27] Figures courtesy of R. Gran, University of Minnesota Duluth

[28] R. A. Smith and E. J. Moniz, Nucl. Phys. B 43 (1972) 605

[29] O. Benhar et al., Phys. Rev. D 72 (2005) 053005

[30] G. Battistoni et al., hep-ph/9801426 (1998)

[31] M. Kordosky, Nucl. Phys. B 159 (2006) 223-228

[32] H. Budd, A. Bodek and J. Arrington, hep-ex/0308005 (2003)

[33] H. Budd, A. Bodek and J. Arrington, hep-ex/0602017 (2005)

[34] K. L. Miller et al., Phys. Rev. D 26 (1982) 537

[35] W. A. Mann et al., Phys. Rev. Lett. 31 (1973) 844

[36] S. J. Barish et al., Phys. Rev. D 16 (1977) 3103

[37] R. Gran et al. [K2K Collaboration], Phys. Rev. D 74 (2006) 052002

[38] A. A. Aguilar-Arevalo et al. [MiniBooNE Collaboration], hep-ex/07060926 (2007)

[39] T. Kitagaki et al., Phys. Rev. D 28 (1983) 436

[40] T. Kitagaki et al., Phys. Rev. D 42 (1990) 1331

[41] S. Boneti et al. [Gargamelle Collaboration], Nuovo Cimento A 38 (1977) 260 
[42] J. Brunner et al. [SKAT Collaboration], Z. Phys. C 45 (1990) 551

[43] R. Kustom et al., Phys. Rev. Lett. 22 (1969) 1014

[44] L. Ahrens et al., Phys. Rev. D 35 (1987) 785

[45] L. Ahrens et al., Phys. Lett. B 202 (1988) 284

[46] V. Bernard et al., J. Phys. G 28 (2002) R1

[47] D. E. Groom et al. [Particle Data Group], Eur. Phys. J C5 (2000) 1

[48] The MINOS Collaboration, The MINOS Technical Design Report, Technical report, Fermilab 1999

[49] The MINOS Collaboration, The NuMI Beam Technical Design Report, Technical report, Fermilab 1999

[50] Images and photographs courtesy of Fermilab Visual Media Services

[51] R. Zwaska, Ph.D. thesis, University of Texas at Austin (2005)

[52] P. Adamson et al. [MINOS Collaboration], Phys. Rev. D 73 (2006) 072002

[53] T. Raufer, Ph.D. thesis, University College, University of Oxford (2007)

[54] T. Cundiff et al., Trans. Nucl. Sci. 53 (2006) 1347

[55] E. Lisi, A. Marrone and D. Montanino, Phys. Rev. Lett. 85 (2000) 1166

[56] V. D. Barger et al., Phys. Rev. Lett. 82 (1999) 2640

[57] M. Apollonio et al. [CHOOZ Collaboration], Phys. Lett. B 466 (1999) 415

[58] M. Acciarri et al. [L3 Collaboration], Phys. Lett. B 431 (1998) 199

[59] A. Aguilar et al. [LSND Collaboration], Phys. Rev. D 64 (2001) 112007

[60] A. Aguilar-Arevalo et al. [MiniBooNE Collaboration], Phys. Rev. Lett. 98 (2007) 231801

[61] J. J. Hartnell, Ph.D. thesis, St. John's College, University of Oxford (2005) 
[62] D. E. Groom, N. V. Mokhov and S. I. Striganov, Atom. Data Nucl. Data Tabl. 78 (2001) 183

[63] P. L. Vahle, Ph.D. thesis, University of Texas at Austin (2004)

[64] M. A. Kordosky, Ph.D. thesis, University of Texas at Austin (2004)

[65] R. Brun et al., GEANT Detector Description and Simulation Tool, CERN Program Library Long Writeup W5013 (1994)

[66] H. Gallagher, Nucl. Phys. Proc. Suppl. 112 (2002) 188-194

[67] D. Rein and L. M. Seghal, Ann. Phys. 133 (1981) 79

[68] A. Bodek and U. K. Yang, AIP Conf. Proc. 721 (2004) 358

[69] T. Yang et al., AIP Conf. Proc. 967 (2007) 269

[70] Z. Koba, H. B. Nielsen and P. Olesen, Nucl. Phys. B 40 (1972) 317

[71] T. Sjostrand, S. Mrenna and P. Skands, JHEP 0605 (2006) 026

[72] R. Merenyi et al., Phys. Rev. D 45 (1992) 743

[73] C. Zeitnitz and T. A. Gabriel, Nucl. Instrum. Meth A 349 (1994) 106

[74] A. Blake, MINOS Internal Note 2720 (2007)

[75] S. Kopp et al., MINOS Internal Note 2965 (2007)

[76] L. A. Ahrens et al., Phys. Rev. D 34 (1986) 75

[77] M. Bonesini et al., Eur. Phys. J. C 20 (2001) 13

[78] A. Mislivec, MINOS Internal Note 3207 (2007)

[79] R. Barlow and C. Beeston, Comp. Phys. Comm 77 (1993) 219-228

[80] J. Thomas, MINOS Internal Note 3993 (2007) 


\section{A Apparent Fit Bias Studies}

This appendix gives further details of the studies into the apparent fit bias that was presented in chapter 7. In this previous discussion the apparent bias in the fit results, illustrated in a number of fits to a mock data sample, was shown not to be an intrinsic bias in the fit method but rather due to statistical differences between the various MC samples used. It was also mentioned that the full MC sample had been sub-divided into four statistically independant sub-samples and basic $M_{A}^{Q E L}$ shape-only fit results using two of these sub-samples (one as mock data with the other as MC and vice versa) were presented.

These results used a number of randomly inputted mock data $M_{A}^{Q E L}$-scales and showed that (a) there was a consistent bias in the extracted $M_{A}^{Q E L}$-scale no matter what the input mock data scale factor and (b) that when the two sub-samples were reversed then the apparent fit bias was reversed no matter what the input mock data scale factor. These two observations implied that it was statistical differences between the sub-samples that were being 'fit-out' in addition to the input mock data $M_{A}^{Q E L}$-scale and that the fit was not inherently biased.

Of the four statistically independant sub-samples only two were used in the results shown in chapter 7. This appendix will show the statistical differences that drive the observed bias in the fit results when these two sub-samples are used and, in addition, present results obtained using the remaining two MC sub-samples.

Of the four sub-samples one was always used in the fits as the MC whilst each of the remaining three were used as sets of mock data. Shape-only fits were performed, using the $M_{A}^{Q E L}$-scale as the single fitted parameter (as described in the apparent bias study section of chapter 7), to the nominal $Q_{Q E L}^{2}$ distributions of each of the three mock data samples and figures 108 through 110 show the un-smeared nominal, smeared nominal and best fit $\mathrm{MC} Q_{Q E L}^{2}$ distributions along with the mock data $Q_{Q E L}^{2}$ distribution for each of the mock data samples. Note that mock data sample 3 was used to give the results shown in figure 85 in chapter 7. 



Figure 108: Reconstructed $Q_{Q E L}^{2}$ for the MC and mock data used in the apparent bias studies with mock data sub-sample 1. The left-hand figure shows the mock data in black points, the smeared nominal MC in blue and the best fit MC in red. The pink curve shows the un-smeared nominal MC and it can be seen, as expected, that this is almost identical to smeared MC. The three MC curves are each normalised to the integral of the mock data sample. The right-hand figure shows the ratios of the mock data to each of the three MC curves.
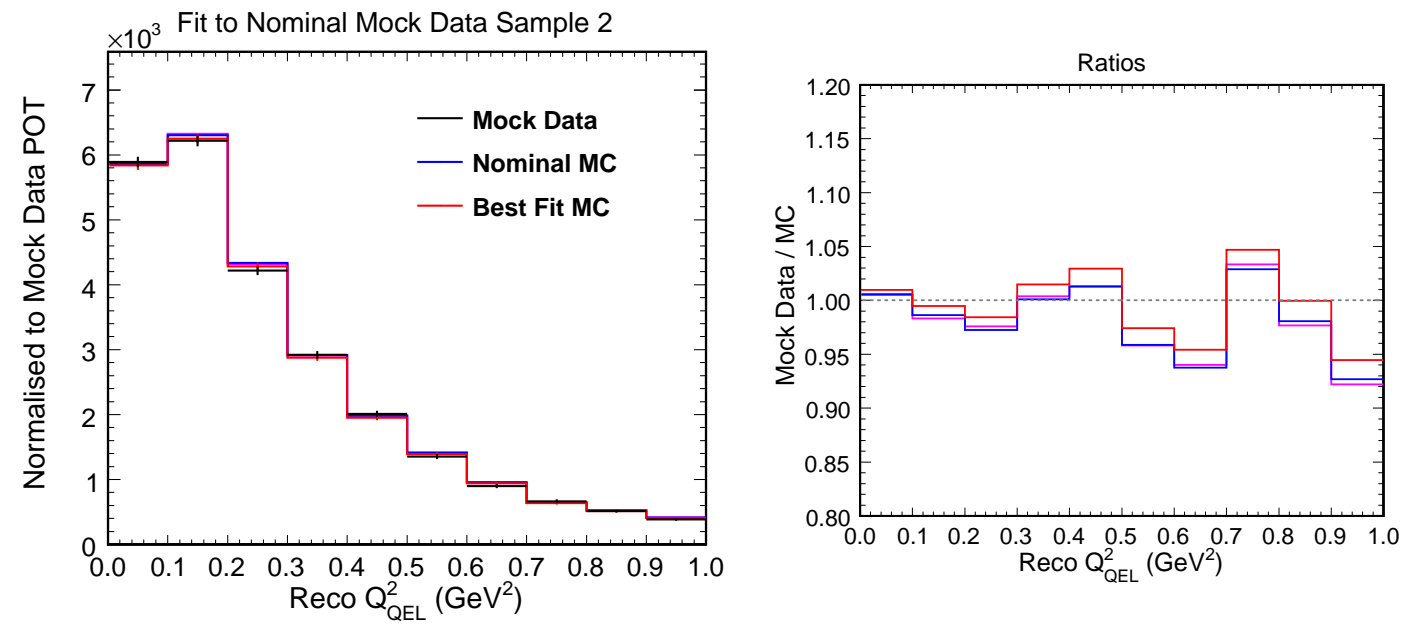

Figure 109: Reconstructed $Q_{Q E L}^{2}$ for the $M C$ and mock data used in the apparent bias studies with mock data sub-sample 2. The left-hand figure shows the mock data in black points, the smeared nominal MC in blue and the best fit MC in red. The pink curve shows the un-smeared nominal MC and it can be seen, as expected, that this is almost identical to smeared MC. The three MC curves are each normalised to the integral of the mock data sample. The right-hand figure shows the ratios of the mock data to each of the three MC curves. 

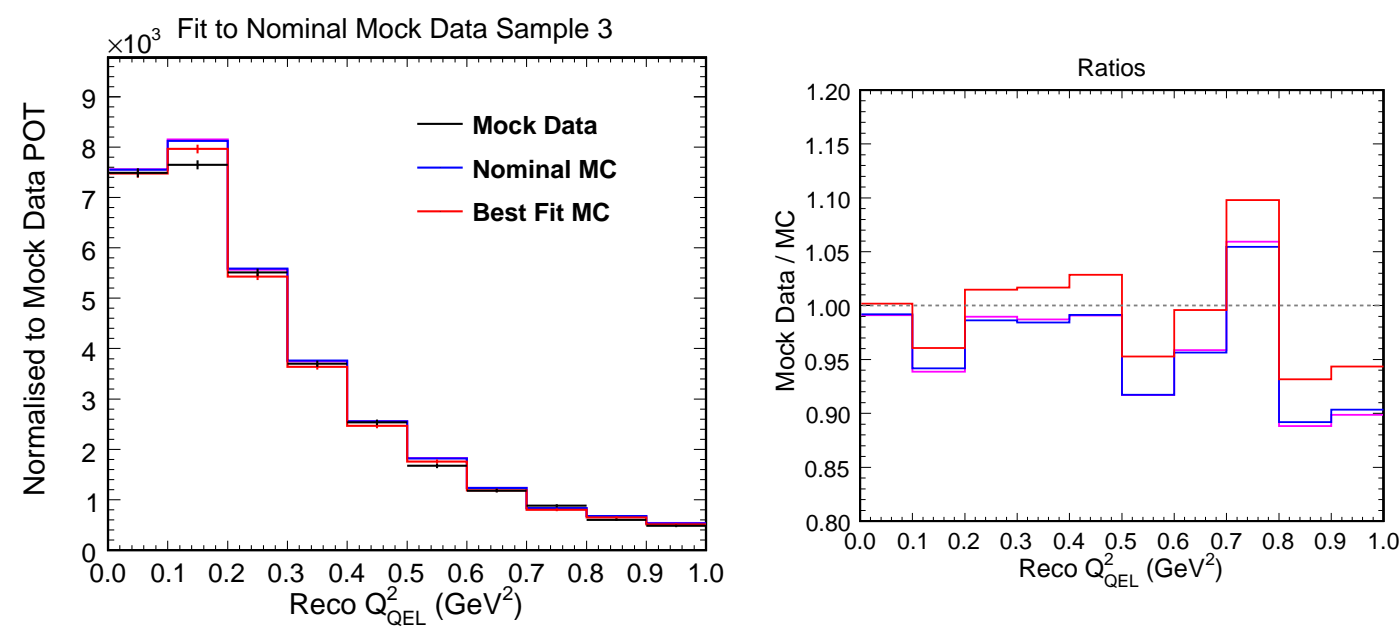

Figure 110: Reconstructed $Q_{Q E L}^{2}$ for the MC and mock data used in the apparent bias studies with mock data sub-sample 3. The left-hand figure shows the mock data in black points, the smeared nominal MC in blue and the best fit MC in red. The pink curve shows the un-smeared nominal MC and it can be seen, as expected, that this is almost identical to smeared MC. The three MC curves are each normalised to the integral of the mock data sample. The right-hand figure shows the ratios of the mock data to each of the three MC curves.

In figures 108 through 110 the black mock data sample points can be compared to the blue nominal MC curves to see the statistical differences in the $Q_{Q E L}^{2}$ distributions between these sub-samples. The blue nominal MC curves can also be compared to the red best fit MC curves to see that in each case the single fit parameter, the $M_{A}^{Q E L}$-scale, has been modified from it's nominal value of unity in order to force better agreement between the shape of the $\mathrm{MC}$ and the mock data. Table 22 lists the $\chi^{2}$ values of each of the mock data samples as compared to the nominal and best fit MC as well as the value of the $M_{A}^{Q E L}$-scale at best fit. It can be seen that in all cases the best fit MC results in a smaller $\chi^{2}$ value than the nominal MC.

In the case of mock data sample 1 the largest difference is seen in the $0.1 \rightarrow 0.2 \mathrm{GeV}^{2}$ bin where the nominal MC lies above the nominal mock data. The fit to mock data sample 1 has decreased the value of $M_{A}^{Q E L}$ in order to reduce the MC contents in this bin and provide a better description of the mock data. For mock data sample 2 the MC can be seen to have a deficit in both the $0.1 \rightarrow 0.2$ and $0.2 \rightarrow 0.3 \mathrm{GeV}^{2}$ bins and again the fit has reduced the value of $M_{A}^{Q E L}$ to force better agreement between the two samples. The most prominent difference between the 


\begin{tabular}{|c|c|c|c|}
\hline Mock Data Sample & $\chi^{2}$ to Nominal MC & $\chi^{2}$ to Best Fit MC & Best Fit $M_{A}^{Q E L}$-Scale \\
\hline \hline 1 & 13.474 & 10.756 & 0.981 \\
\hline 2 & 14.303 & 10.452 & 0.976 \\
\hline 3 & 61.656 & 35.447 & 0.945 \\
\hline
\end{tabular}

Table 22: Fit results for bias studies using four statistically independant sub-samples of MC. One of the sub-samples is used as a MC sample and the other three as mock data samples. The table shows the calculated $\chi^{2}$ between the nominal MC sample and each of the mock data samples as well as the $\chi^{2}$ value after a single parameter fit is performed for the $M_{A}^{Q E L}$-scale parameter. The final column shows the value of the axial-mass scale factor at best fit.

$\mathrm{MC}$ and the various mock data sets is shown in figure 110 where mock data sample 3 can be seen to have a significant excess in the $0.1 \rightarrow 0.2 \mathrm{GeV}^{2}$ bin. As with the previous mock data samples, the fit uses a reduction in $M_{A}^{Q E L}$ to 'fit-out' some of this difference but in this case the reduction is larger and corresponds to a decrease of almost $6 \%$.

Chapter 7 showed the results of 20 single parameter fits using the MC sample and mock data sample 3 where the first fit corresponded to the nominal mock data and in the remaining 19 fits the value of $M_{A}^{Q E L}$ had been randomly selected (between $\pm 20 \%$ ) and applied to the mock data prior to fitting. In all 20 cases the best fit $M_{A}^{Q E L}$-scales were seen to have a consistent bias relative to the input mock data $M_{A}^{Q E L}$-scale and furthermore when the samples were reversed (with mock data sample 3 used as MC and the MC sample used as mock data) the bias in the extracted $M_{A}^{Q E L}$-scales was reversed. Figures 111 through 113 show similar results for mock data samples 1 and 2 along with the plots shown previously for mock data sample 3 .

Given the assertation that the apparent fit bias is due to statistical differences between the four sub-samples, figures 111 through 113 mights be expected to show error bars for the best fit minus truth points (where the errors correspond to those on the fitted $M_{A}^{Q E L}$-scale value returned by the MIGRAD routine) that are large enough to encompass zero. This is not the case because, as seen in figures 108 through 110, there are differences between the samples that fall outside the size of the statistical errors on the bin contents. In particular the $0.1 \rightarrow 0.2 \mathrm{GeV}^{2}$ bin in figure 110 shows a (somewhat unlucky) $\sim 3 \sigma$ deviation between the samples and explains the larger distance between the error bars and lines at zero in figure 113. 

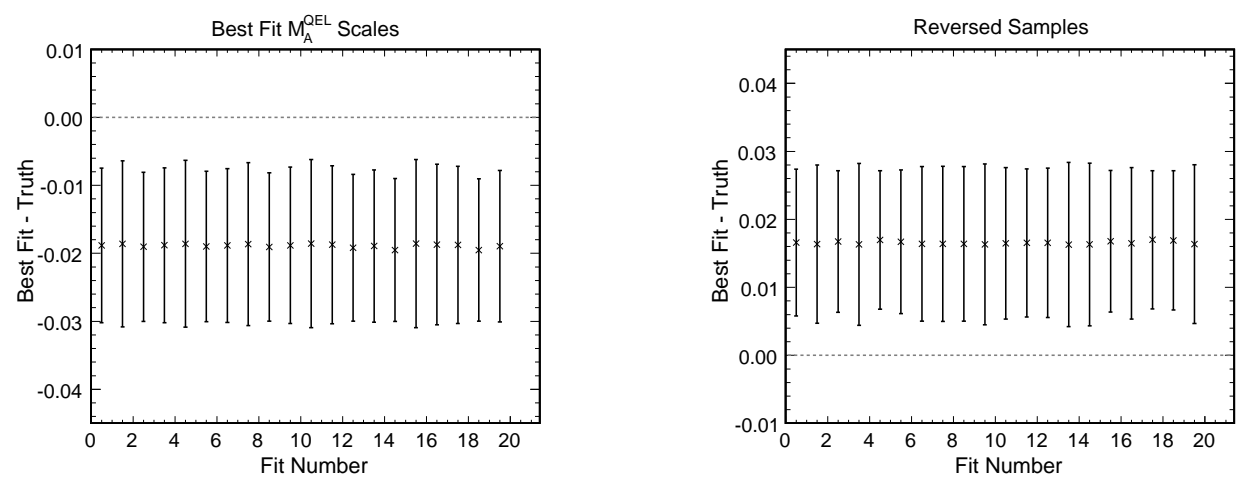

Figure 111: Best fit minus truth $M_{A}^{Q E L}$-scale values for 20 single parameter fits (indexed on the x-axis) to mock data sample 1 with randomly generated $M_{A}^{Q E L}$-scale input values and 20 fits with the mock data and MC samples reversed.
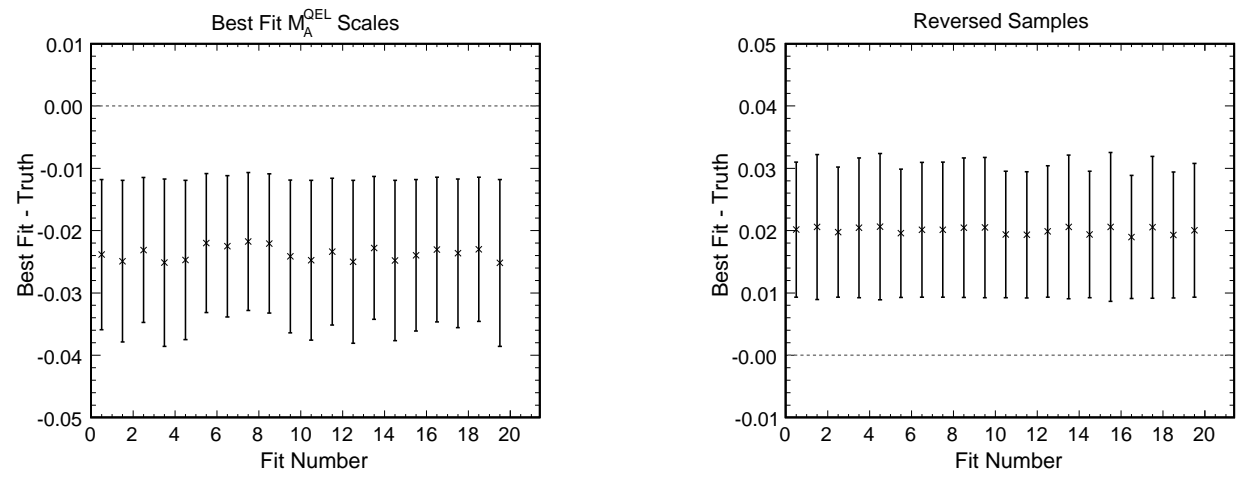

Figure 112: Best fit minus truth $M_{A}^{Q E L}$-scale values for 20 single parameter fits (indexed on the $x$-axis) to mock data sample 2 with randomly generated $M_{A}^{Q E L}$-scale input values and 20 fits with the mock data and MC samples reversed.


Figure 113: Best fit minus truth $M_{A}^{Q E L}$-scale values for 20 single parameter fits (indexed on the $x$-axis) to mock data sample 3 with randomly generated $M_{A}^{Q E L}$-scale input values and 20 fits with the mock data and MC samples reversed. 
One further thing to note when considering figures 111 through 113 is that the apparent bias is not perfectly reversed when the samples are reversed and, in particular, slightly smaller upward shifts in $M_{A}^{Q E L}$ are found in the reversed sample fits. This is not evidence for a smaller, but real, bias in the fit procedure but rather reflects the fact that whilst changes to the single fit parameter, the $M_{A}^{Q E L}$-scale, increase or decrease the weights given to the true $\mathrm{v}_{\mu}-\mathrm{CC}$ QEL events present in the MC QEL-like sample they do not affect the non-QEL background events. As such, an identical magnitude change in $M_{A}^{Q E L}$ will produce slightly different results depending upon the direction of the change.

This appendix has presented further evidence to justify the statement that the $M_{A}^{Q E L}$ fitting procedure that has been developed is not intrinsically biased but rather that the apparent biases seen in the mock data fit results are due to differences between the various MC sub-samples used. In this appendix four statistically independant sub-samples of MC were shown to have differences in their $Q_{Q E L}^{2}$ distributions and it was shown that:

- Fits to the various mock data sub-samples do not result in a consistent bias across the samples but rather the size and direction of the apparent bias is in accordance with 'fitting-out' the differences between each mock data sample and the MC.

- Fits to the same mock data sample but with different inputted $M_{A}^{Q E L}$-scale values give a consistent bias indicating that the bias does not depend upon the value of $M_{A}^{Q E L}$ applied to the mock data before each fit but rather is directly related to the differences between the underlying nominal mock data sample and the MC.

- For each set of fits to a single mock data sample the apparent bias is reversed under a reversal of the mock data and $\mathrm{MC}$ samples showing again that the observed bias in the fit results is a function of the underlying differences between each mock data sample and the MC rather than being an inherent feature of the fitting process. 


\section{B Correlation Coeffi cients}

This appendix presents correlation coefficiencts for the two headline fits to the data presented in chapter 8 . The correlation coefficients reported below are those returned by the HESSE routine and, following the discussion presented in chapter 7 , they should not be considered accurate to better than $10-20 \%$. The correlation coefficients are presented in matrix form with rows and columns labelled by the numbers $1-9$ which denote the fit parameters according to the following:

1. $M_{A}^{Q E L}$-scale

2. $E_{\mu}$-scale

3. $E_{\text {had }}$-offset

4. Inelastic background scale

5. $0<E_{v}^{\text {true }}<2 \mathrm{GeV}$ flux factor

6. $2 \leq E_{v}^{\text {true }}<3 \mathrm{GeV}$ flux factor

7. $3 \leq E_{v}^{\text {true }}<4 \mathrm{GeV}$ flux factor

8. $4 \leq E_{v}^{\text {true }}<6 \mathrm{GeV}$ flux factor

9. $6 \leq E_{\mathrm{v}}^{\text {true }}<10 \mathrm{GeV}$ flux factor

where, as with the tables of results from chapter 8, the two higher energy flux factors have been neglected.

Corrleation Coeffi cicents for Headline Fit I to the ND Data

\begin{tabular}{c|cccc} 
& 1 & 2 & 3 & 4 \\
\hline 1 & 1.0 & -0.65 & 0.44 & -0.48 \\
2 & -0.65 & 1.0 & -0.06 & -0.06 \\
3 & 0.44 & -0.06 & 1.0 & -0.22 \\
4 & -0.48 & -0.06 & -0.22 & 1.0
\end{tabular}


Corrleation Coeffi cicents for Headline Fit II to the ND Data

\begin{tabular}{c|ccccccccc} 
& 1 & 2 & 3 & 4 & 5 & 6 & 7 & 8 & 9 \\
\hline 1 & 1.0 & -0.41 & 0.38 & -0.45 & 0.20 & 0.03 & -0.06 & -0.02 & 0.09 \\
2 & -0.41 & 1.0 & 0.05 & 0.05 & 0.26 & 0.30 & 0.09 & -0.07 & 0.01 \\
3 & 0.38 & 0.05 & 1.0 & 0.02 & 0.17 & 0.17 & 0.13 & 0.07 & 0.06 \\
4 & -0.45 & 0.05 & 0.02 & 1.0 & -0.87 & -0.78 & -0.67 & -0.61 & -0.65 \\
5 & 0.20 & 0.26 & 0.17 & -0.87 & 1.0 & 0.86 & 0.75 & 0.63 & 0.66 \\
6 & 0.03 & 0.30 & 0.17 & -0.78 & 0.86 & 1.0 & 0.72 & 0.67 & 0.66 \\
7 & -0.06 & 0.09 & 0.13 & -0.67 & 0.75 & 0.72 & 1.0 & 0.56 & 0.60 \\
8 & -0.02 & -0.07 & 0.07 & -0.61 & 0.63 & 0.67 & 0.56 & 1.0 & 0.50 \\
9 & 0.09 & 0.01 & 0.06 & -0.65 & 0.66 & 0.66 & 0.60 & 0.50 & 1.0
\end{tabular}

